Supporting Information

\title{
Scandium in Neutral and Positively Charged Ammonia Complexes: Balancing Between $\mathrm{Sc}^{2+}$ and $\mathrm{Sc}^{3+}$
}

\author{
Shahriar N. Khan and Evangelos Miliordos* \\ Department of Chemistry and Biochemistry, Auburn University, \\ Auburn, AL 36849, USA
}

* To whom correspondence should be addressed. E-mail: emiliord@auburn.edu 


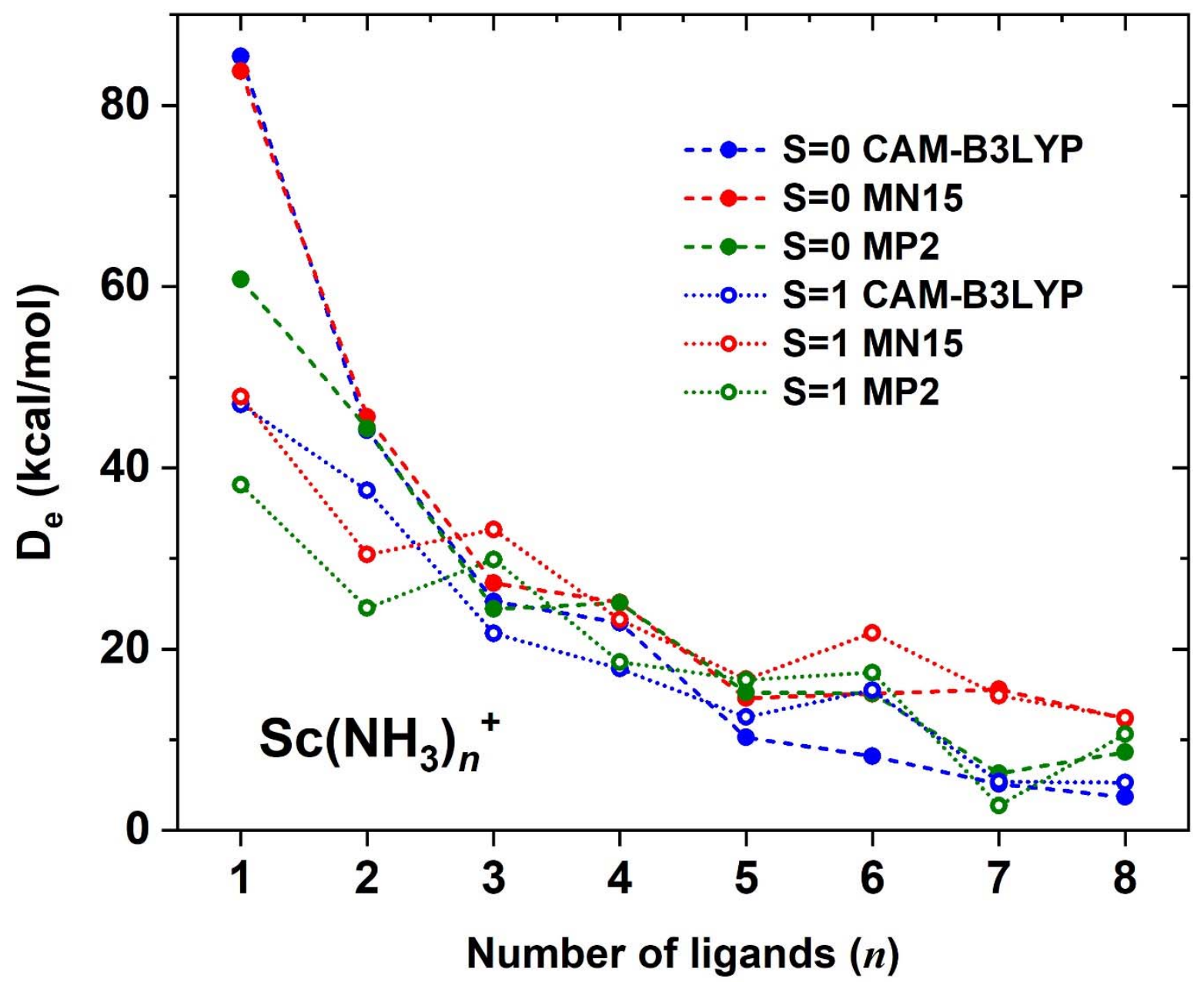

Figure S1: Detachment energy $(\mathrm{De}, \mathrm{kcal} / \mathrm{mol})$ of one ammonia ligand from the singlet $(\mathrm{S}=0)$ and triplet $(\mathrm{S}=1)$ state of $\mathrm{Sc}\left(\mathrm{NH}_{3}\right)_{n}^{+}$at the CAM-B3LYP, MN15, and MP2 levels of theory as a function of $n$. Equation (1) of the manuscript is used. The binding energy of $\operatorname{Sc}\left(\mathrm{NH}_{3}\right)^{+}$is with respect to $\mathrm{Sc}^{+}\left({ }^{3} \mathrm{D} ; 4 \mathrm{~s}^{1} 3 \mathrm{~d}^{1}\right)$ and $\mathrm{Sc}^{+}\left({ }^{1} \mathrm{~S} ; 4 \mathrm{~s}^{2}\right)$ states. 


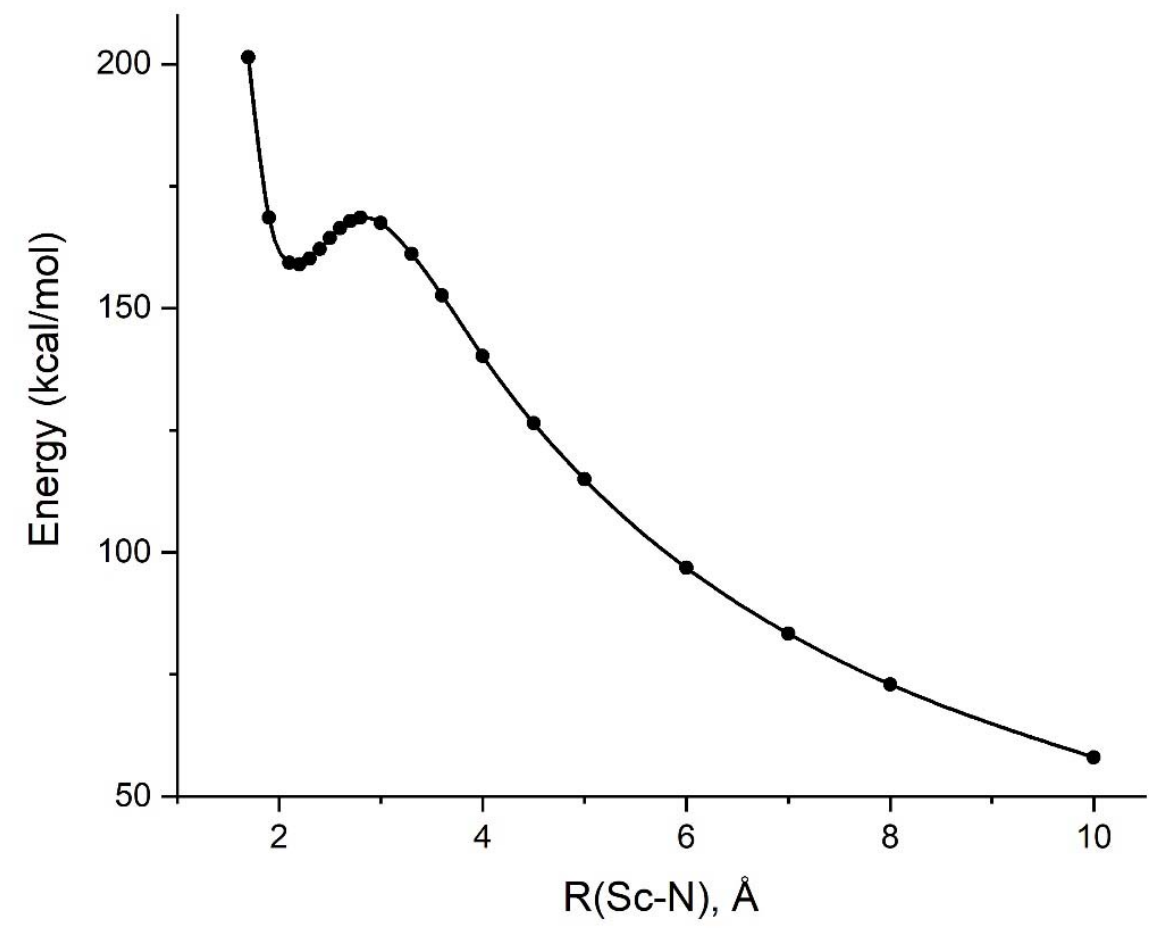

Figure S2: Potential energy curves of $\mathrm{Sc}\left(\mathrm{NH}_{3}\right)^{3+}$ as a function of the Sc-N distance. The zero of the energy axis is set equal to the energy of $\mathrm{Sc}^{2+}+\mathrm{NH}_{3}{ }^{+}$. 
Table S1: Excitation energies (eV) for $\mathrm{Sc}^{+}$at the CASSCF and CASPT2 levels of theory.

\begin{tabular}{cccccccc}
\hline State & CASSCF $^{a}$ & CASPT2 $^{a}$ & MRCI $^{\mathrm{a}, b}$ & C-CASPT2 $^{\mathrm{a}, b}$ & C-MRCI $^{\mathrm{a}, b}$ & ${\text { C-MRCI }+Q^{\mathrm{a}, b}}^{\text {Expt }^{d}}$ \\
\hline${ }^{3} \mathrm{D}\left(4 \mathrm{~s}^{1} 3 \mathrm{~d}^{1}\right)$ & 0.000 & 0.000 & 0.000 & 0.000 & 0.000 & 0.000 & 0.000 \\
${ }^{1} \mathrm{D}\left(4 \mathrm{~s}^{1} 3 \mathrm{~d}^{1}\right)$ & 0.474 & 0.311 & 0.261 & 0.294 & 0.321 & 0.254 & 0.302 \\
${ }^{3} \mathrm{~F}\left(3 \mathrm{~d}^{2}\right)$ & 0.471 & 0.573 & 0.666 & 0.422 & 0.500 & 0.590 & 0.596 \\
${ }^{1} \mathrm{D}\left(3 \mathrm{~d}^{2}\right)$ & 1.742 & 1.389 & 1.484 & 1.217 & 1.347 & 1.307 & 1.344 \\
${ }^{1} \mathrm{~S}\left(4 \mathrm{~s}^{2}\right)$ & 2.374 & 2.149 & 1.950 & 1.678 & 1.696 & 1.447 & 1.442 \\
${ }^{3} \mathrm{P}\left(3 \mathrm{~d}^{2}\right)$ & 1.680 & 1.617 & 1.638 & 1.368 & 1.449 & 1.482 & 1.491 \\
${ }^{1} \mathrm{G}\left(3 \mathrm{~d}^{2}\right)$ & 2.023 & 1.925 & 1.920 & 1.646 & 1.741 & 1.748 & 1.755 \\
${ }^{1} \mathrm{~S}\left(3 \mathrm{~d}^{2}\right)$ & 4.666 & 3.598 & 3.287 & 3.347 & 3.528 & 3.169 & 3.205 \\
\hline
\end{tabular}

\begin{tabular}{ccccc}
\hline State & CASSCF $^{c}$ & CASPT2 $^{c}$ & C-CASPT2 $^{c, b}$ & Expt $^{d}$ \\
\hline${ }^{3} \mathrm{D}\left(4 \mathrm{~s}^{1} 3 \mathrm{~d}^{1}\right)$ & 0.000 & 0.000 & 0.000 & 0.000 \\
${ }^{1} \mathrm{D}\left(4 \mathrm{~s}^{1} 3 \mathrm{~d}^{1}\right)$ & 0.387 & 0.298 & 0.316 & 0.302 \\
${ }^{3} \mathrm{~F}\left(3 \mathrm{~d}^{2}\right)$ & 0.480 & 0.583 & 0.423 & 0.596 \\
${ }^{1} \mathrm{D}\left(3 \mathrm{~d}^{2}\right)$ & 1.582 & 1.452 & 1.247 & 1.344 \\
${ }^{1} \mathrm{~S}\left(4 \mathrm{~s}^{2}\right)$ & 1.857 & 1.943 & 1.664 & 1.442 \\
${ }^{3} \mathrm{P}\left(3 \mathrm{~d}^{2}\right)$ & 1.644 & 1.617 & 1.360 & 1.491 \\
${ }^{1} \mathrm{G}\left(3 \mathrm{~d}^{2}\right)$ & 2.032 & 1.932 & 1.645 & 1.755 \\
${ }^{1} \mathrm{~S}\left(3 \mathrm{~d}^{2}\right)$ & & & & 3.205 \\
\hline
\end{tabular}

${ }^{a}$ CASSCF active space: two electrons in six orbitals $(4 s+3 d)$. Basis set: cc-pVTZ.

${ }^{b}$ The $3 s^{2} 3 p^{6}$ electrons are also correlated. Basis set: cc-pwCVTZ.

${ }^{c}$ CASSCF active space: two electrons in nine orbitals $(4 s+3 d+4 p)$. Basis set: cc-pVTZ.

${ }^{d} \mathrm{M}_{\mathrm{J}}$ averaged values; Ref. 30 of the manuscript. 
Table S2: Electronic energies (a.u.) for $\mathrm{Sc}^{+}\left({ }^{3} \mathrm{D} ; 4 \mathrm{~s}^{1} 3 \mathrm{~d}^{1}\right)$ and $\mathrm{Sc}^{+}\left({ }^{1} \mathrm{~S} ; 4 \mathrm{~s}^{2}\right)$ states, and their difference $\Delta \mathrm{E}(\mathrm{eV})$ at different levels of theory and basis sets.

\begin{tabular}{cccc}
\hline State & $\mathrm{E}\left({ }^{3} \mathrm{D}\right)$ & $\mathrm{E}\left({ }^{1} \mathrm{~S}\right)$ & $\Delta \mathrm{E}$ \\
\hline HF/cc-pVTZ & -759.461600 & -759.540263 & 2.141 \\
MP2/cc-pVTZ & -759.486196 & -759.543591 & 1.562 \\
MP2/aug-cc-pVTZ & -759.486513 & -759.543633 & 1.554 \\
MP2/aug-cc-pV5Z & -759.487695 & -759.544499 & 1.546 \\
C-MP2/aug-cc-pwCV5Z & -759.803695 & -759.868736 & 1.770 \\
C-CCSD(T)/aug-cc-pwCV5Z & -759.835571 & -759.895220 & 1.623 \\
CASSCF/cc-pVTZ ${ }^{a}$ & -759.525332 & -759.438099 & 2.374 \\
CASPT2/cc-pVTZ ${ }^{a}$ & -759.535705 & -759.456736 & 2.149 \\
C-CASPT2/cc-pwCVTZ $^{a, b}$ & -759.850103 & -759.788450 & 1.678 \\
CASSCF/cc-pVTZ $^{c}$ & -759.525553 & -759.457292 & 1.857 \\
CASPT2/cc-pVTZ $^{c}$ & -759.535872 & -759.464453 & 1.943 \\
C-CASPT2/cc-pwCVTZ $^{c, b}$ & -759.850084 & -759.788949 & 1.664 \\
Expt $^{d}$ & & & 1.442 \\
\hline
\end{tabular}

${ }^{a}$ CASSCF active space: two electrons in six orbitals $(4 s+3 d)$.

${ }^{b}$ The $3 s^{2} 3 p^{6}$ electrons are also correlated.

${ }^{c}$ CASSCF active space: two electrons in nine orbitals $(4 s+3 d+4 p)$.

${ }^{d} \mathrm{M}_{\mathrm{J}}$ averaged values; Ref. 30 of the manuscript. 
Table S3: Electronic energies (a.u.) for the $\mathrm{Sc}\left(\mathrm{NH}_{3}\right)_{\mathrm{n}=1-8^{3+}}$ species optimized at CAM-B3LYP, MN15, MP2 and C-MP2 combined with the cc-pVTZ $(\mathrm{Sc}, \mathrm{N})$ /aug-cc-pVTZ(H) basis sets for the former three methods and cc-pwCVTZ(Sc)/cc-pVTZ(N)/aug-cc-pVTZ(H) for C-MP2.

\begin{tabular}{ccccc}
\hline$n$ & CAM-B3LYP & MN15 & MP2 & C-MP2 \\
\hline 0 & -759.004887 & -758.970857 & -758.214557 & -758.473971 \\
1 & -815.851738 & -815.762949 & -814.918870 & -815.197983 \\
2 & -872.613948 & -872.473298 & -871.566202 & -871.855205 \\
3 & -929.335711 & -929.145268 & -928.182071 & -928.477445 \\
4 & -986.030562 & -985.792304 & -984.774553 & -985.075102 \\
5 & -1042.680255 & -1042.401753 & -1041.328260 & -1041.631073 \\
6 & -1099.314958 & -1099.001881 & -1097.868764 & -1098.173175 \\
7 & -1155.916417 & -1155.567947 & -1154.375081 & \\
8 & -1212.506447 & -1212.123815 & -1210.872295 & \\
\hline
\end{tabular}


Table S4: Cartesian coordinates $(\AA)$ for the $\mathrm{Sc}\left(\mathrm{NH}_{3}\right)_{\mathrm{n}=1-8^{3+}}$ species optimized at CAM-B3LYP, MN15, and MP2 combined with the cc-pVTZ $(\mathrm{Sc}, \mathrm{N})$ aug-cc-pVTZ $(\mathrm{H})$ basis sets.

\begin{tabular}{|c|c|c|c|c|c|c|c|c|c|c|c|}
\hline \multicolumn{4}{|c|}{ CAM-B3LYP } & \multicolumn{4}{|c|}{ MN15 } & \multicolumn{4}{|c|}{ MP2 } \\
\hline \multicolumn{12}{|c|}{$\mathrm{Sc}\left(\mathrm{NH}_{3}\right) 1^{3+}$} \\
\hline $\mathrm{N}$ & -1.361698 & -0.000001 & -0.000015 & $\mathrm{~N}$ & -1.362833 & -0.000001 & -0.000011 & $\mathrm{~N}$ & -1.392946 & -0.000000 & -0.000021 \\
\hline $\mathrm{H}$ & -1.788878 & 0.841515 & -0.463763 & $\mathrm{H}$ & -1.793029 & 0.826269 & -0.489367 & $\mathrm{H}$ & -1.829931 & 0.823535 & -0.474033 \\
\hline $\mathrm{H}$ & -1.788916 & -0.822432 & -0.496790 & $\mathrm{H}$ & -1.793045 & -0.836982 & -0.470803 & $\mathrm{H}$ & -1.829940 & -0.822371 & -0.476042 \\
\hline $\mathrm{H}$ & -1.788965 & -0.019063 & 0.960616 & $\mathrm{H}$ & -1.793054 & 0.010722 & 0.960229 & $\mathrm{H}$ & -1.829955 & -0.001158 & 0.950191 \\
\hline $\mathrm{Sc}$ & 0.709459 & -0.000001 & 0.000002 & $\mathrm{Sc}$ & 0.710427 & -0.000000 & 0.000001 & $\mathrm{Sc}$ & 0.725736 & -0.000000 & 0.000002 \\
\hline \multicolumn{12}{|c|}{$\mathrm{Sc}\left(\mathrm{NH}_{3}\right)_{2}{ }^{3+}$} \\
\hline $\mathrm{N}$ & 1.793618 & 0.556654 & 0.000086 & $\bar{N}$ & 1.811572 & 0.542308 & 0.000080 & $\mathrm{~N}$ & 1.835188 & 0.564237 & 0.000080 \\
\hline $\mathrm{H}$ & 2.654093 & -0.026598 & 0.000083 & $\mathrm{H}$ & 2.667903 & -0.049835 & -0.000016 & $\mathrm{H}$ & 2.702381 & -0.004648 & 0.000021 \\
\hline $\mathrm{H}$ & 1.914862 & 1.182644 & -0.819135 & $\mathrm{H}$ & 1.944219 & 1.168688 & -0.819270 & $\mathrm{H}$ & 1.961871 & 1.192627 & -0.813693 \\
\hline $\mathrm{H}$ & 1.914796 & 1.182534 & 0.819401 & $\mathrm{H}$ & 1.944239 & 1.168450 & 0.819608 & $\mathrm{H}$ & 1.961861 & 1.192444 & 0.813995 \\
\hline $\mathrm{N}$ & -1.792653 & 0.557434 & 0.000058 & $\mathrm{~N}$ & -1.810676 & 0.542908 & & $\mathrm{~N}$ & -1.834682 & 0.564629 & 0040 \\
\hline $\mathrm{H}$ & -2.407455 & 0.375274 & 162 & $\mathrm{H}$ & 5737 & 0.356815 & & $\mathrm{H}$ & 803 & 0.394247 & 693 \\
\hline $\mathrm{H}$ & -1.668826 & 1.586898 & 0.00 & $\mathrm{H}$ & -1.700141 & 1.575921 & 29 & $\mathrm{H}$ & -1. & 1.592574 & 371 \\
\hline $\mathrm{H}$ & -2.407229 & 0.375836 & -0.81 & $\mathrm{H}$ & -2.426545 & 0.357267 & -0.8 & $\mathrm{H}$ & 584 & 0.394744 & \\
\hline $\mathrm{Sc}$ & -0.000333 & -0.594057 & -0.00 & $\mathrm{Sc}$ & -0.000439 & -0.579705 & & $\mathrm{Sc}$ & 225 & -0.603050 & \\
\hline \multicolumn{12}{|c|}{$\mathrm{Sc}\left(\mathrm{NH}_{3}\right) 3^{3+}$} \\
\hline $\mathrm{H}$ & -1.009428 & 2.113528 & 21 & $\mathrm{H}$ & -0.6 & -2.213546 & & $\mathrm{H}$ & 52 & -2.167958 & 25 \\
\hline $\mathrm{N}$ & -0.655391 & 2.007782 & 21 & $\mathrm{~N}$ & -0.8 & -1.939814 & & $\mathrm{~N}$ & & -2.030554 & 155 \\
\hline $\mathrm{H}$ & 0.075957 & 2.732097 & 0.1 & $\mathrm{H}$ & -1.8 & -2.029729 & & $\mathrm{H}$ & 0. & -2.795120 & 395 \\
\hline $\mathrm{H}$ & -1.423424 & 2.371223 & -0.3 & $\mathrm{H}$ & -0.43 & -2.736678 & -0.3 & $\mathrm{H}$ & 23 & -2.331851 & 924 \\
\hline $\mathrm{N}$ & -1.411158 & -1.571379 & 0.20 & $\mathrm{~N}$ & 2.09 & 0.256363 & & $\mathrm{~N}$ & 605 & 1.706306 & 456 \\
\hline $\mathrm{H}$ & -1.322217 & -1.934106 & 1.163389 & $\mathrm{H}$ & 2.261959 & 0.514361 & 80 & $\mathrm{H}$ & 1.279970 & 2.118042 & 1.096455 \\
\hline $\mathrm{H}$ & -2.403876 & -1.297939 & 0.129778 & $\mathrm{H}$ & 2.679717 & -0.583532 & 0.065576 & $\mathrm{H}$ & 2.365222 & 1.493191 & 0.072591 \\
\hline $\mathrm{H}$ & -1.346090 & -2.416448 & -0.388878 & $\mathrm{H}$ & 2.588014 & 0.990419 & -0.3 & $\mathrm{H}$ & 374 & 2.518683 & $-0.4 c$ \\
\hline $\mathrm{N}$ & 6533 & -0.436243 & & $\mathrm{~N}$ & -1.2 & 1.683339 & & $\mathrm{~N}$ & & 0.324225 & 465 \\
\hline $\mathrm{H}$ & 588 & -0.183816 & & $\mathrm{H}$ & -1.5 & 1.703189 & & $\mathrm{H}$ & & 0.05 & 189 \\
\hline $\mathrm{H}$ & 2.328167 & -1.431653 & 90 & $\mathrm{H}$ & -0.836614 & 2.612156 & & $\mathrm{H}$ & 298 & 1.301332 & 482 \\
\hline $\mathrm{H}$ & 2.765564 & 0.048018 & -0.38 & $\mathrm{H}$ & -2.153916 & 1.742875 & -0.3 & $\mathrm{H}$ & -2.800927 & -0.187813 & -0.468617 \\
\hline $\mathrm{Sc}$ & 0.000042 & -0.000097 & -0.3 & $\mathrm{Sc}$ & 0.000208 & 0.000060 & & $\mathrm{Sc}$ & -0.000026 & 0.000013 & -0.2 \\
\hline \multicolumn{12}{|c|}{$\mathrm{Sc}\left(\mathrm{NH}_{3}\right)_{4}^{3+}$} \\
\hline Sc & -0.000010 & 0.000021 & & Sc & 0.000357 & -0.000251 & & Sc & 06 & $\overline{0.00}$ & 031 \\
\hline $\mathrm{N}$ & -1.767397 & -0.604825 & & $\mathrm{~N}$ & -0.9 & 0.395752 & & $\mathrm{~N}$ & & -0.62 & 543 \\
\hline $\mathrm{H}$ & -2.641691 & -0.675751 & 64 & $\mathrm{H}$ & -1.7 & 1.029012 & & $\mathrm{H}$ & 26 & -0.65 & 88 \\
\hline $\mathrm{H}$ & -2.003572 & 0.032581 & 90 & $\mathrm{H}$ & -1.292170 & -0.441251 & -2.4 & $\mathrm{H}$ & 220 & -0.018837 & 367 \\
\hline $\mathrm{N}$ & 1.816726 & -0.127216 & 1.260025 & $\mathrm{~N}$ & 1.879409 & -1.128244 & -0.290468 & $\mathrm{~N}$ & -1.823421 & -0.219645 & -1.302003 \\
\hline $\mathrm{H}$ & 2.074249 & -1.080770 & 1.546187 & $\mathrm{H}$ & 2.633856 & -0.596647 & -0.748653 & $\mathrm{H}$ & -2.062059 & -1.186122 & -1.557780 \\
\hline $\mathrm{H}$ & 1.760124 & 0.389012 & 2.147591 & $\mathrm{H}$ & 1.787373 & -1.974573 & -0.870986 & $\mathrm{H}$ & -1.770800 & 0.259734 & -2.209677 \\
\hline $\mathrm{N}$ & 0.214212 & -1.349988 & -1.742350 & $\mathrm{~N}$ & 0.440496 & 1.919027 & 1.005796 & $\mathrm{~N}$ & -0.217274 & -1.304649 & 1.821755 \\
\hline $\mathrm{H}$ & -0.538094 & -1.273441 & -2 & $\mathrm{H}$ & -0.391369 & 2.450219 & & $\mathrm{H}$ & 156 & -1.190375 & 9609 \\
\hline $\mathrm{H}$ & 0.232540 & -2.351390 & & $\mathrm{H}$ & 0.971749 & 2.592031 & & $\mathrm{H}$ & -0.2 & -2.315628 & 420 \\
\hline $\mathrm{N}$ & -0.263527 & 2.081992 & -0.7 & $\mathrm{~N}$ & -1.386716 & -1.186055 & & $\mathrm{~N}$ & 26 & 2.148811 & 846 \\
\hline $\mathrm{H}$ & 0.461772 & 2.408534 & -1.3 & $\mathrm{H}$ & -1.088831 & -1.305952 & 39 & $\mathrm{H}$ & -0.525993 & 2.492688 & 1.263098 \\
\hline $\mathrm{H}$ & -1.143031 & 2.255702 & -1.210972 & $\mathrm{H}$ & -2.336585 & -0.794433 & & $\mathrm{H}$ & 1.077573 & 2.368081 & 1.147484 \\
\hline $\mathrm{H}$ & 2.671302 & 0.236173 & 0.818299 & $\mathrm{H}$ & 2.313520 & -1.472053 & 0.578470 & $\mathrm{H}$ & -2.695657 & 0.139953 & -0.894157 \\
\hline $\mathrm{H}$ & -1.683739 & -1.522658 & 1.645590 & $\mathrm{H}$ & -0.308369 & 0.830709 & -2.657786 & $\mathrm{H}$ & 1.777490 & -1.560477 & -1.578377 \\
\hline $\mathrm{H}$ & -0.262407 & 2.791262 & 0.037356 & $\mathrm{H}$ & -1.544252 & -2.149755 & 0.919428 & $\mathrm{H}$ & 0.210756 & 2.832730 & -0.130418 \\
\hline $\mathrm{H}$ & 1.072658 & -1.209426 & -2.290780 & $\mathrm{H}$ & 0.997991 & 1.834593 & 1.868227 & $\mathrm{H}$ & -1.083878 & -1.165120 & 2.356519 \\
\hline \multicolumn{12}{|c|}{$\mathrm{Sc}\left(\mathrm{NH}_{3}\right) 5^{3+}$} \\
\hline $\mathrm{N}$ & -0.003921 & -1.736805 & 1.432685 & $\mathrm{~N}$ & 0.003428 & -1.987239 & -1.040712 & $\mathrm{~N}$ & 0.003770 & -1.775116 & -1.439340 \\
\hline
\end{tabular}




\begin{tabular}{|c|c|c|c|c|c|c|c|c|c|c|c|}
\hline & & & & & & & & & & & \\
\hline $\mathrm{H}$ & .807638 & -1.781969 & 2.056208 & $\mathrm{H}$ & -0.809683 & -2.159073 & -1.643353 & $\mathrm{H}$ & -0.804865 & -1.831929 & -2.065890 \\
\hline $\mathrm{H}$ & -0.804994 & -1.765861 & 2.070618 & $\mathrm{H}$ & 0.806508 & -2.146999 & -1.659955 & $\mathrm{H}$ & 0.802499 & -1.816785 & -2.079689 \\
\hline $\mathrm{H}$ & -0.017117 & -2.656306 & 0.978019 & $\mathrm{H}$ & 0.015921 & -2.792618 & -0.402280 & $\mathrm{H}$ & 0.016316 & -2.694771 & -0.985019 \\
\hline $\mathrm{N}$ & -2.295539 & 0.003000 & -0.000442 & $\mathrm{~N}$ & 2.286043 & 0.001894 & 0.000889 & $\mathrm{~N}$ & 2.316157 & 0.002808 & 0.000701 \\
\hline $\mathrm{H}$ & -2.726120 & -0.899255 & -0.223393 & $\mathrm{H}$ & 2.714436 & -0.843058 & 0.396140 & $\mathrm{H}$ & 2.753177 & -0.893822 & 234838 \\
\hline $\mathrm{H}$ & -2.724738 & 0.261974 & 0.892812 & $\mathrm{H}$ & 2.714358 & 0.082740 & -0.928469 & $\mathrm{H}$ & 2.752180 & 0.249208 & -0.893126 \\
\hline $\mathrm{H}$ & -2.723916 & 0.648028 & -0.670981 & $\mathrm{H}$ & & 7006 & & $\mathrm{H}$ & & & 780 \\
\hline $\mathrm{N}$ & 2.295736 & -0.002486 & 0.001763 & $\mathrm{~N}$ & -2.286328 & -0.002790 & -0.000615 & $\mathrm{~N}$ & -2.316338 & & -0.001600 \\
\hline $\mathrm{H}$ & 2.724356 & -0.905655 & -0.221301 & $\mathrm{H}$ & -2.713143 & -0.848441 & 0.394863 & $\mathrm{H}$ & -2.751631 & 9841 & 0.232578 \\
\hline $\mathrm{H}$ & 2.726289 & 0.641854 & -0.668041 & $\mathrm{H}$ & -2.715389 & 0.761671 & 429 & $\mathrm{H}$ & 3465 & 737 & 657737 \\
\hline $\mathrm{H}$ & 2.724717 & 0.255039 & 0.895547 & $\mathrm{H}$ & -2.714216 & 0.076622 & -0.930297 & $\mathrm{H}$ & -2.752007 & 2687 & -0.895970 \\
\hline $\mathrm{Sc}$ & 0.000112 & 0.000051 & 0015 & $\mathrm{Sc}$ & -0.000122 & 0.000166 & 0342 & $\mathrm{Sc}$ & 0090 & 0035 & -0.000055 \\
\hline $\mathrm{N}$ & 0.002829 & 2.109867 & 0.7 & $\mathrm{~N}$ & 975 & 588 & & $\mathrm{~N}$ & 2584 & 4612 & 6362 \\
\hline $\mathrm{H}$ & 0.00 & $2.1^{\prime}$ & & $\mathrm{H}$ & & & & $\mathrm{H}$ & & & 990 \\
\hline $\mathrm{H}$ & 0.80 & $2.6^{\prime}$ & 0.4 & $\mathrm{H}$ & -0.8 & 524 & & $\mathrm{H}$ & 768 & & 126 \\
\hline $\mathrm{H}$ & -0.80 & 42 & 78 & $\mathrm{H}$ & 75 & & 70 & $\mathrm{H}$ & & & 606 \\
\hline $\mathrm{N}$ & 0.000720 & -0.373690 & -2.22 & $\mathrm{~N}$ & -0.0 & 260 & & $\mathrm{~N}$ & 869 & 987 & 6694 \\
\hline $\mathrm{H}$ & 0.806341 & -0.903122 & -2.566834 & $\mathrm{H}$ & -0.808641 & 5222 & 2.689841 & $\mathrm{H}$ & 3900 & 5645 & 615227 \\
\hline $\mathrm{H}$ & -0.806444 & -0.900091 & -2.5 & $\mathrm{H}$ & & 941 & & $\mathrm{H}$ & 602 & 698 & 6301 \\
\hline $\mathrm{H}$ & 0.002701 & 0.47 & -2.7 & $\mathrm{H}$ & 452 & 1.047799 & & $\mathrm{H}$ & & & 469 \\
\hline \multicolumn{12}{|c|}{$\mathrm{Sc}\left(\mathrm{NH}_{3}\right)_{6}{ }^{3+}$} \\
\hline $\mathrm{Sc}$ & -0.0 & 63 & & $\mathrm{Sc}$ & 196 & 333 & & $\mathrm{Sc}$ & 012 & 005 & 012 \\
\hline $\mathrm{N}$ & -1.344242 & 1.768643 & -0.6 & $\mathrm{~N}$ & -1.0 & -1. & -1 & $\mathrm{~N}$ & 23 & 46 & 815 \\
\hline $\mathrm{H}$ & -0.960120 & 2.696168 & -0.462424 & $\mathrm{H}$ & -1.9 & -1.4 & -1.5 & $\mathrm{H}$ & 34 & 580 & 8020 \\
\hline $\mathrm{H}$ & -2.267978 & 1.791985 & -0.2 & $\mathrm{H}$ & -1.4 & -2.4 & -0. & $\mathrm{H}$ & 831 & 822 & 843 \\
\hline $\mathrm{N}$ & -1.350650 & -0.325611 & & $\mathrm{~N}$ & & 007 & 84 & $\mathrm{~N}$ & 357 & 710 & 920 \\
\hline $\mathrm{H}$ & -0.9 & -0.9 & & $\mathrm{H}$ & & & & $\mathrm{H}$ & & & \\
\hline $\mathrm{H}$ & -2.2 & -0.7 & 1.6 & $\mathrm{H}$ & & -0 & & $\mathrm{H}$ & & & \\
\hline $\mathrm{N}$ & 1.3 & -1.8 & & $\mathrm{~N}$ & & & & $\mathrm{~N}$ & & & 860 \\
\hline $\mathrm{H}$ & 0.9 & -2.4 & 26 & $\mathrm{H}$ & 36 & 16 & & $\mathrm{H}$ & 310 & 33 & 116 \\
\hline $\mathrm{H}$ & 2.265456 & -1.604701 & 0.8 & I & 289 & 848 & 14 & $\mathrm{H}$ & 5185 & 865 & 247 \\
\hline $\mathrm{N}$ & 1.349133 & 0.53 & -1.8 & $\mathrm{~N}$ & 505 & 946 & 1. & $\mathrm{~N}$ & 1777 & 341 & 389 \\
\hline $\mathrm{H}$ & 2.271807 & 0.0 & -1.7 & $\mathrm{H}$ & 513 & 440 & & $\mathrm{H}$ & 377 & 293 & 504 \\
\hline $\mathrm{H}$ & 0.966437 & 0.28 & & $\mathrm{H}$ & & -0 . & & $\mathrm{H}$ & & 362 & 332 \\
\hline $\mathrm{H}$ & -1. & & & $\mathrm{H}$ & & & & $\mathrm{H}$ & 69 & 46 & 84 \\
\hline $\mathrm{H}$ & -1 . & & & $\mathrm{H}$ & & & & $\mathrm{H}$ & & & 311 \\
\hline $\mathrm{H}$ & & & -1.8 & $\mathrm{H}$ & & -0 . & & $\mathrm{H}$ & & 96 & 109 \\
\hline $\mathrm{H}$ & 709 & -2.40 & -0.2 & $\mathrm{H}$ & 531 & 35 & 49 & $\mathrm{H}$ & 1592 & 860 & 737 \\
\hline $\mathrm{N}$ & -1.334686 & -1.451590 & 460 & $\mathrm{~N}$ & -0.879375 & 267 & -1 & $\mathrm{~N}$ & 6191 & 908 & -0.076112 \\
\hline $\mathrm{H}$ & -1.542446 & -2.32 & -0.7 & $\mathrm{H}$ & -0.8 & 515 & -2 & $\mathrm{H}$ & 00 & 104 & 887 \\
\hline $\mathrm{H}$ & -0.942042 & -1.751435 & -2.1 & $\mathrm{H}$ & -0.3 & 993 & 69 & $\mathrm{H}$ & 600 & 621 & 481 \\
\hline $\mathrm{H}$ & -2.2 & -1.0 & & $\mathrm{H}$ & & & & $\mathrm{H}$ & & 28 & 388 \\
\hline $\mathrm{N}$ & & & & $\mathrm{N}$ & & & & $\mathrm{N}$ & & 116 & 514 \\
\hline $\mathrm{H}$ & & & & $\mathrm{H}$ & & & & $\mathrm{H}$ & & & 446 \\
\hline $\mathrm{H}$ & & & & $\mathrm{H}$ & & & & $\mathrm{H}$ & & 93 & 727 \\
\hline $\mathrm{H}$ & 639 & 325 & & $\mathrm{H}$ & 808 & -1.999615 & 729 & $\mathrm{H}$ & 073 & 695 & -0 . \\
\hline \multicolumn{12}{|c|}{$\mathrm{Sc}\left(\mathrm{NH}_{3}\right) 7^{3+}$} \\
\hline $\mathrm{Sc}$ & 0.037160 & 519 & 63 & $\mathrm{Sc}$ & 7525 & -0. & & $\mathrm{Sc}$ & 2206 & 7624 & 0057 \\
\hline $\mathrm{N}$ & -0.400306 & 1.561721 & 3033 & $\mathrm{~N}$ & -0.057032 & 2748 & 036 & $\mathrm{~N}$ & 0.215854 & -1.613520 & 1.731748 \\
\hline $\mathrm{H}$ & -1.15 & 36 & & $\mathrm{H}$ & 318 & & & $\mathrm{H}$ & & 33 & 236 \\
\hline $\mathrm{H}$ & -0.648122 & 03 & & $\mathrm{H}$ & 208 & & 79 & $\mathrm{H}$ & 617 & -2.575833 & 863 \\
\hline $\mathrm{N}$ & 1.962792 & & & $\mathrm{~N}$ & 2.138021 & 0.978660 & 0.394759 & $\mathrm{~N}$ & -2.125332 & -1.161941 & -0.000952 \\
\hline $\mathrm{H}$ & & 1.258102 & & $\mathrm{H}$ & 8785 & 93 & 37 & $\mathrm{H}$ & -2.730122 & -0.969177 & 0.799501 \\
\hline $\mathrm{H}$ & 1.767650 & 2.389547 & 0.002822 & $\mathrm{H}$ & 2.114304 & 1.830567 & 0.957948 & $\mathrm{H}$ & -2.055161 & -2.180103 & -0.002385 \\
\hline $\mathrm{N}$ & -0.395892 & -1.481685 & -1.793579 & $\mathrm{~N}$ & -0.264927 & -0.093437 & -2.297964 & $\mathrm{~N}$ & 0.567062 & 1.440272 & -1.803800 \\
\hline
\end{tabular}




\begin{tabular}{|c|c|c|c|c|c|c|c|c|c|c|c|}
\hline & & & & & & & & & & & \\
\hline $\mathrm{H}$ & 239487 & .284701 & -2.330293 & $\mathrm{H}$ & -1.056411 & 0.437545 & -2.664822 & $\mathrm{H}$ & 1.379242 & 1.150770 & -2.349024 \\
\hline $\mathrm{H}$ & -0.504670 & -2.459100 & -1.521143 & $\mathrm{H}$ & -0.382163 & -1.028992 & -2.691403 & $\mathrm{H}$ & 0.794734 & 2.399960 & -1.537833 \\
\hline $\mathrm{N}$ & -2.346825 & -0.067438 & -0.000132 & $\mathrm{~N}$ & -2.322661 & 0.333197 & 0.003845 & $\mathrm{~N}$ & 2.358205 & -0.198047 & 0.000097 \\
\hline $\mathrm{H}$ & -2.727778 & -1.013545 & -0.001855 & $\mathrm{H}$ & -2.825409 & -0.422065 & -0.465594 & $\mathrm{H}$ & 2.852110 & 0.695723 & 0.002315 \\
\hline $\mathrm{H}$ & -2.795150 & 0.377037 & 0.799806 & $\mathrm{H}$ & -2.708761 & 0.358715 & 0.949368 & $\mathrm{H}$ & .762692 & -0.692077 & 5910 \\
\hline $\mathrm{H}$ & 2.579149 & 1.260274 & -0.801959 & $\mathrm{H}$ & 2.567825 & 1.267337 & -0.487522 & $\mathrm{H}$ & -2.730564 & -0.966949 & -0.800526 \\
\hline $\mathrm{H}$ & 0.39 & 924 & 2.34 & $\mathrm{H}$ & 0.688 & -0.326385 & 2.738751 & $\mathrm{H}$ & -0.577624 & 1581 & 45 \\
\hline $\mathrm{H}$ & -2.795491 & 0.380094 & -0.798166 & $\mathrm{H}$ & -2.689126 & 1.181936 & -0.429563 & $\mathrm{H}$ & 2.763220 & -0.688356 & -0.797750 \\
\hline $\mathrm{H}$ & 0.343038 & -1.503780 & -2.497554 & $\mathrm{H}$ & 0.549698 & 0.293179 & -2.779 & $\mathrm{H}$ & 909 & 1.559603 & 0782 \\
\hline $\mathrm{N}$ & 1.908027 & -1.471767 & -0.001525 & $\mathrm{~N}$ & 1.644684 & -1.633197 & -0.553976 & $\mathrm{~N}$ & 7834 & 2135 & 0.000944 \\
\hline $\mathrm{H}$ & 2.528167 & -1.364971 & 0.801960 & $\mathrm{H}$ & 2.087667 & -1.985689 & 0.297858 & $\mathrm{H}$ & -2.372328 & 1.664032 & 0.800998 \\
\hline $\mathrm{H}$ & 2.528598 & -1.362688 & -0.804370 & $\mathrm{H}$ & 2.422643 & -1.307144 & -1.130967 & $\mathrm{H}$ & -2.371568 & 5557 & -0.799764 \\
\hline $\mathrm{H}$ & 1.680434 & -2.465606 & -0.003000 & $\mathrm{H}$ & 1.308989 & -2.471773 & -1.0 & $\mathrm{H}$ & -1.403287 & 6332 & 2015 \\
\hline $\mathrm{N}$ & -0.400988 & 1.566910 & -1.71 & $\mathrm{~N}$ & -0.260782 & 2.311518 & -0.3 & $\mathrm{~N}$ & 787 & 101 & 4843 \\
\hline $\mathrm{H}$ & 0.3 & 1.7 & -2.3 & $\mathrm{H}$ & & & -0.6 & $\mathrm{H}$ & & & 5689 \\
\hline $\mathrm{H}$ & -0.6 & $2.4 \mathrm{C}$ & -1.3 & $\mathrm{H}$ & -0.6 & 25 & & $\mathrm{H}$ & 63 & 36 & 113 \\
\hline $\mathrm{H}$ & -1.16 & 1.32 & -2.3 & $\mathrm{H}$ & -0.9 & 77 & -1.1 & $\mathrm{H}$ & 78 & 11 & 002 \\
\hline $\mathrm{N}$ & -0.396466 & -1.486699 & 1.789268 & $\mathrm{~N}$ & -0.9 & -2.099828 & 0.5 & $\mathrm{~N}$ & 110 & 980 & 5720 \\
\hline $\mathrm{H}$ & -0.504638 & -2.463482 & 1.514341 & $\mathrm{H}$ & -1.230035 & -2.601064 & -0.3 & $\mathrm{H}$ & 0.7 & 7166 & 1.542587 \\
\hline $\mathrm{H}$ & 0.342091 & -1.510193 & $2.4 \mathrm{C}$ & $\mathrm{H}$ & -0.306751 & -2.751016 & & $\mathrm{H}$ & -0.1 & & 550 \\
\hline $\mathrm{H}$ & -1.2 & -1.29 & 2.3 & $\mathrm{H}$ & -1.7 & -2.076022 & 1.1 & $\mathrm{H}$ & & & \\
\hline & & & & & & & & & & & \\
\hline $\mathrm{H}$ & 82 & 2.6 & 1.2 & $\mathrm{H}$ & 34 & -2.6 & & $\mathrm{H}$ & & 81 & 862 \\
\hline $\mathrm{N}$ & 1.16 & 1.655568 & 1.3 & $\mathrm{H}$ & 637 & $-2 .($ & 1.2 & $\mathrm{H}$ & 500 & 40 & 637 \\
\hline $\mathrm{H}$ & 2.470543 & 1.230457 & -1.197781 & $\mathrm{~N}$ & 163 & 2054 & 1.3 & $\mathrm{~N}$ & 874 & 433 & 629 \\
\hline $\mathrm{H}$ & 2.176325 & 1.692052 & 1.204966 & $\mathrm{H}$ & -2.183232 & -1.797311 & 0.95 & $\mathrm{H}$ & 0.121751 & -2.768300 & 1.217612 \\
\hline $\mathrm{H}$ & 1.065089 & 1.511166 & 2.344362 & $\mathrm{H}$ & 0.740418 & -1.514176 & & $\mathrm{H}$ & 583 & 2633 & 588 \\
\hline $\mathrm{H}$ & 0.3 & 2.73 & -1.2 & $\mathrm{~N}$ & -1.8 & -0 . & & $\mathrm{N}$ & 08 & & \\
\hline $\mathrm{H}$ & -0.3 & 1.82 & $-2.3+>$ & $\mathrm{H}$ & & & & $\mathrm{H}$ & & & \\
\hline $\mathrm{N}$ & 1.9 & 0.3 & -1.3 & $\mathrm{H}$ & -2.7 & -0.2 & & $\mathrm{H}$ & -1.4 & -2 & 781 \\
\hline $\mathrm{N}$ & -0.34 & 1.95 & -1.3 & $\mathrm{Sc}$ & -0.0 & 000 & 0.0 & $\mathrm{Sc}$ & 000 & 000 & 058 \\
\hline $\mathrm{H}$ & 2.617616 & -0.863846 & 1.204099 & $\mathrm{H}$ & 2.723723 & 0.297538 & 1.2 & $\mathrm{H}$ & 398 & 246 & 5437 \\
\hline $\mathrm{H}$ & 2.730814 & -0.354339 & -1.210702 & $\mathrm{~N}$ & 1.878254 & 0.867647 & 1.28 & $\mathrm{~N}$ & 0.502508 & 750 & 1.347218 \\
\hline $\mathrm{H}$ & 1.821388 & 0.319523 & -2.344252 & $\mathrm{H}$ & 1.692842 & 0.971937 & 2.28 & $\mathrm{H}$ & 764 & 298 & 2.354152 \\
\hline $\mathrm{H}$ & -1.230302 & 2.470367 & -1.198075 & $\mathrm{H}$ & -0.740129 & 4187 & 74 & $\mathrm{H}$ & 407 & 868 & 676 \\
\hline Sc & -0.000001 & 0.000000 & & $\mathrm{~N}$ & & & & $\mathrm{~N}$ & & & 25 \\
\hline $\mathrm{N}$ & 1.6 & -1 . & & $\mathrm{H}$ & & & & $\mathrm{H}$ & & 22 & 45 \\
\hline $\mathrm{H}$ & -1.6 & & & $\mathrm{H}$ & & & & $\mathrm{H}$ & & & 355 \\
\hline $\mathrm{H}$ & 1. & -1.0 & 2.3 & $\mathrm{H}$ & -0.2 & 24 & 1.3 & $\mathrm{H}$ & -2.3 & 03 & 396 \\
\hline $\mathrm{N}$ & -1.655570 & 1.16 & 1.3 & $\mathrm{H}$ & -0.20 & -2.699391 & -1.2 & $\mathrm{H}$ & 2.0 & 4426 & -1.215950 \\
\hline $\mathrm{H}$ & 1.691976 & -2.176371 & 1.2 & $\mathrm{H}$ & -1.675381 & -2.040584 & -1.3 & $\mathrm{H}$ & 348 & -2.690388 & -1.217513 \\
\hline $\mathrm{H}$ & -1.511028 & 1.065455 & 2.34 & $\mathrm{~N}$ & -0.68 & -1.811822 & -1.38 & $\mathrm{~N}$ & 20 & -1.750984 & -1.347538 \\
\hline $\mathrm{H}$ & -1.065237 & -1.510920 & 2.344453 & $\mathrm{H}$ & 2.147471 & -1.74 & -1.1 & $\mathrm{H}$ & 508 & 4041 & 6844 \\
\hline $\mathrm{H}$ & -2.6 & 78 & & $\mathrm{H}$ & -0.4 & & & $\mathrm{H}$ & & 398 & 468 \\
\hline $\mathrm{N}$ & -1.1 & -1.6 & & $\mathrm{~N}$ & & & & $\mathrm{~N}$ & & & 576 \\
\hline $\mathrm{H}$ & & -2.6 & & $\mathrm{H}$ & & & & $\mathrm{H}$ & & & \\
\hline $\mathrm{N}$ & -1.5 & -0.3 & -1.3 & $\mathrm{H}$ & & 96 & -1.0 & $\mathrm{H}$ & 58 & 20 & 958 \\
\hline $\mathrm{H}$ & -1.821223 & -0.319784 & -2.344 & $\mathrm{H}$ & -2.815723 & 0.280095 & -1.03 & $\mathrm{H}$ & -1.874549 & -2.0 & -1.216614 \\
\hline $\mathrm{H}$ & -2.470462 & -1.230586 & -1.197 & $\mathrm{~N}$ & -1.9 & 762 & -1.26 & $\mathrm{~N}$ & -1.7 & 322 & -1.347342 \\
\hline $\mathrm{H}$ & -2.176410 & -1.691912 & 1205 & $\mathrm{H}$ & -1.852160 & 0.617106 & -2.27 & $\mathrm{H}$ & -1.608077 & -0.953578 & -2.354266 \\
\hline $\mathrm{H}$ & -2.730728 & 0.354209 & -1.210935 & $\mathrm{H}$ & 0.487070 & 1.598361 & -2.36 & $\mathrm{H}$ & -0.954516 & 1.607163 & -2.354557 \\
\hline $\mathrm{N}$ & 0.340196 & -1.997253 & -1.340707 & $\mathrm{~N}$ & 0.681051 & 1.811819 & -1.388453 & $\mathrm{~N}$ & -1.038320 & 1.750850 & -1.347636 \\
\hline $\mathrm{H}$ & 1.230382 & -2.470503 & -1.197715 & $\mathrm{H}$ & -2.147613 & 352 & -1.1 & $\mathrm{H}$ & -2.690599 & -0.664162 & -1.216578 \\
\hline $\mathrm{H}$ & 0.320126 & -1.820597 & -2.344326 & $\mathrm{H}$ & 212 & 2.04 & -1.3 & $\mathrm{H}$ & -0.6 & 2.690267 & -1.217732 \\
\hline $\mathrm{H}$ & -0.354455 & -2.730476 & -1.211563 & $\mathrm{H}$ & 0.205168 & 2.699389 & -1.223204 & $\mathrm{H}$ & -2.041801 & 1.874304 & -1.215986 \\
\hline
\end{tabular}


Table S5: Harmonic vibrational frequencies $\left(\mathrm{cm}^{-1}\right)$ for the $\mathrm{Sc}\left(\mathrm{NH}_{3}\right)_{\mathrm{n}=1-8}{ }^{3+}$ species optimized at CAM-B3LYP, MN15, and MP2 combined with the cc-pVTZ(Sc,N) aug-cc-pVTZ $(H)$ basis sets.

\begin{tabular}{|c|c|c|}
\hline CAM-B3LYP & MN15 & MP2 \\
\hline \multicolumn{3}{|c|}{$\mathrm{Sc}\left(\mathrm{NH}_{3}\right)_{1}{ }^{3+}$} \\
\hline 513 & 508 & 513 \\
\hline 761 & 750 & 762 \\
\hline 761 & 751 & 762 \\
\hline 1434 & 1412 & 1439 \\
\hline 1520 & 1495 & 1555 \\
\hline 1520 & 1495 & 1555 \\
\hline 3095 & 3122 & 3154 \\
\hline 3132 & 3168 & 3218 \\
\hline 3133 & 3168 & 3218 \\
\hline \multicolumn{3}{|c|}{$\mathrm{Sc}\left(\mathrm{NH}_{3}\right)_{2}{ }^{3+}$} \\
\hline 11 & 18 & $12 i$ \\
\hline 42 & 39 & 38 \\
\hline 111 & 99 & 96 \\
\hline 471 & 466 & 462 \\
\hline 500 & 499 & 494 \\
\hline 719 & 705 & 716 \\
\hline 720 & 708 & 720 \\
\hline 741 & 728 & 741 \\
\hline 757 & 741 & 751 \\
\hline 1442 & 1418 & 1441 \\
\hline 1451 & 1427 & 1448 \\
\hline 1580 & 1551 & 1598 \\
\hline 1580 & 1551 & 1598 \\
\hline 1584 & 1555 & 1602 \\
\hline 1585 & 1555 & 1602 \\
\hline 3226 & 3244 & 3255 \\
\hline 3245 & 3263 & 3270 \\
\hline 3276 & 3302 & 3326 \\
\hline 3282 & 3306 & 3330 \\
\hline 3293 & 3316 & 3339 \\
\hline 3302 & 3321 & 3344 \\
\hline \multicolumn{3}{|c|}{$\mathrm{Sc}\left(\mathrm{NH}_{3}\right)_{3}{ }^{3+}$} \\
\hline 29 & 38 & 24 \\
\hline 31 & 39 & 24 \\
\hline 40 & 39 & 32 \\
\hline 65 & 50 & 43 \\
\hline 103 & 88 & 90 \\
\hline 103 & 89 & 90 \\
\hline 423 & 422 & 408 \\
\hline 471 & 469 & 468 \\
\hline 471 & 469 & 468 \\
\hline 672 & 657 & 670 \\
\hline 689 & 676 & 690 \\
\hline 690 & 676 & 690 \\
\hline 725 & 709 & 722 \\
\hline 726 & 709 & 722 \\
\hline 726 & 711 & 722 \\
\hline 1438 & 1412 & 1435 \\
\hline 1438 & 1412 & 1435 \\
\hline 1448 & 1422 & 1443 \\
\hline
\end{tabular}




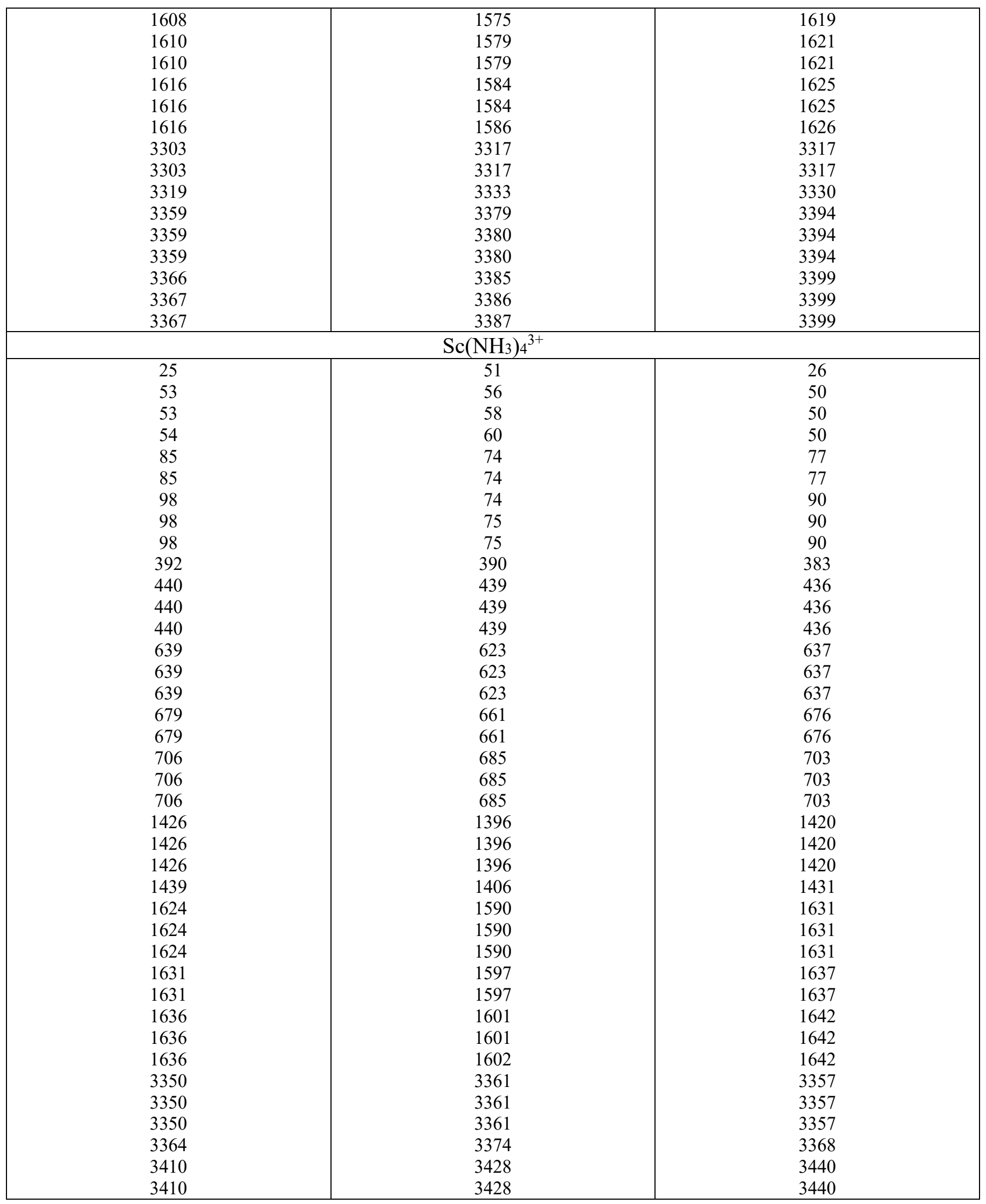




\begin{tabular}{|c|c|c|}
\hline $\begin{array}{l}3410 \\
3410 \\
3410 \\
3413 \\
3413 \\
3413 \\
\end{array}$ & $\begin{array}{l}3429 \\
3429 \\
3429 \\
3431 \\
3431 \\
3431 \\
\end{array}$ & $\begin{array}{l}3440 \\
3440 \\
3440 \\
3442 \\
3442 \\
3442 \\
\end{array}$ \\
\hline \multicolumn{3}{|c|}{$\mathrm{Sc}\left(\mathrm{NH}_{3}\right) 5^{3+}$} \\
\hline 34 & $22 i$ & 26 \\
\hline 34 & 44 & 26 \\
\hline 42 & 45 & 38 \\
\hline 56 & 45 & 52 \\
\hline 56 & 48 & 52 \\
\hline 123 & 105 & 118 \\
\hline 123 & 106 & 118 \\
\hline 145 & 142 & 138 \\
\hline 151 & 148 & 143 \\
\hline 151 & 149 & 143 \\
\hline 157 & 166 & 151 \\
\hline 158 & 167 & 152 \\
\hline 322 & 324 & 324 \\
\hline 373 & 372 & 366 \\
\hline 385 & 387 & 396 \\
\hline 414 & 418 & 413 \\
\hline 414 & 419 & 413 \\
\hline 581 & 552 & 579 \\
\hline 581 & 554 & 579 \\
\hline 608 & 584 & 608 \\
\hline 608 & 585 & 608 \\
\hline 613 & 591 & 609 \\
\hline 676 & 651 & 673 \\
\hline 677 & 654 & 674 \\
\hline 681 & 654 & 678 \\
\hline 686 & 658 & 683 \\
\hline 686 & 658 & 683 \\
\hline 1401 & 1366 & 1396 \\
\hline 1407 & 1369 & 1401 \\
\hline 1412 & 1377 & 1406 \\
\hline 1412 & 1377 & 1406 \\
\hline 1427 & 1388 & 1419 \\
\hline 1639 & 1603 & 1643 \\
\hline 1639 & 1603 & 1643 \\
\hline 1640 & 1604 & 1644 \\
\hline 1640 & 1604 & 1644 \\
\hline 1642 & 1606 & 1646 \\
\hline 1649 & 1612 & 1652 \\
\hline 1660 & 1624 & 1662 \\
\hline 1660 & 1624 & 1662 \\
\hline 1661 & 1625 & 1662 \\
\hline 1661 & 1625 & 1662 \\
\hline 3388 & 3396 & 3389 \\
\hline 3388 & 3396 & 3389 \\
\hline 3390 & 3398 & 3390 \\
\hline 3396 & 3403 & 3394 \\
\hline 3403 & 3410 & 3400 \\
\hline 3448 & 3466 & 3472 \\
\hline
\end{tabular}




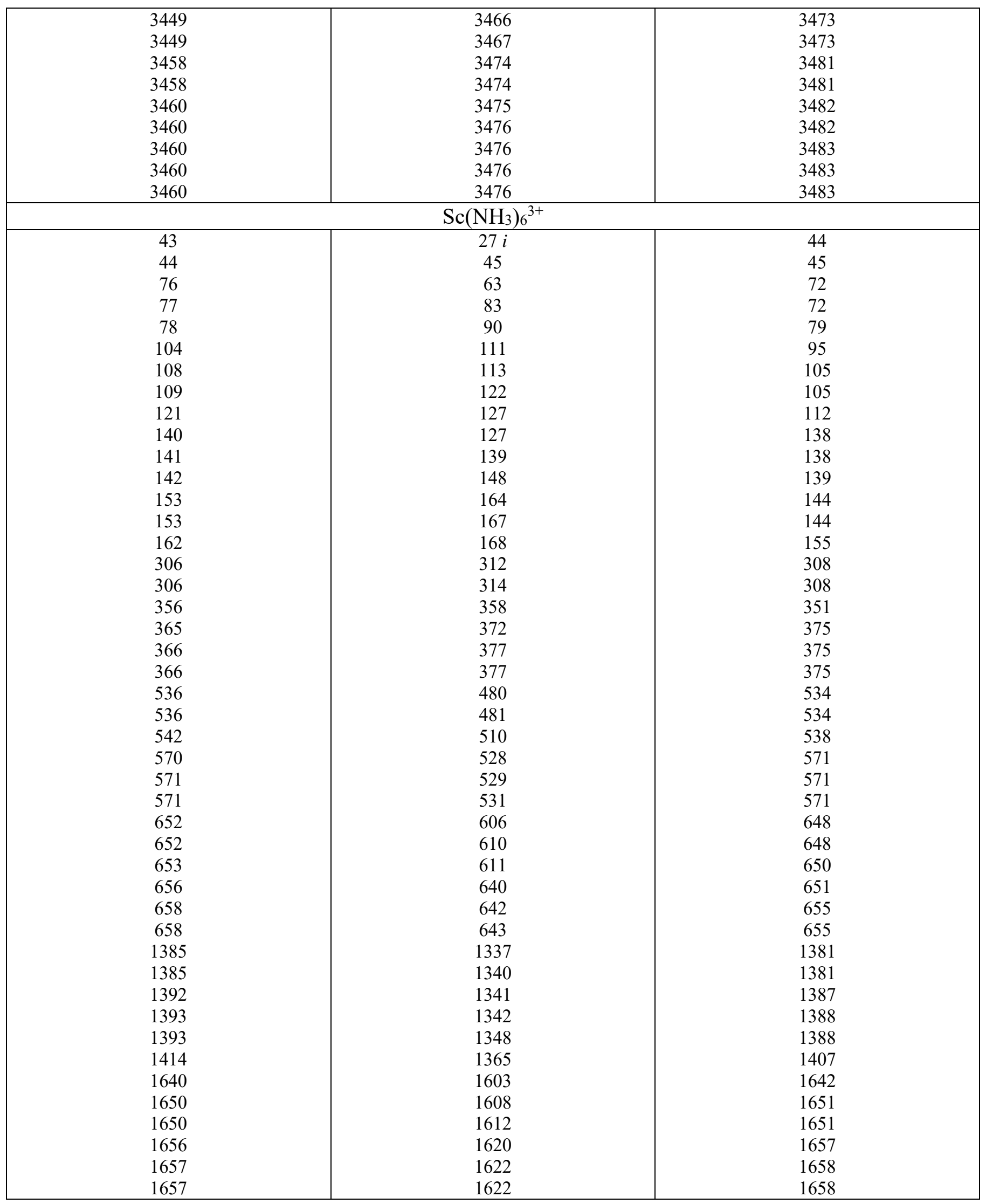




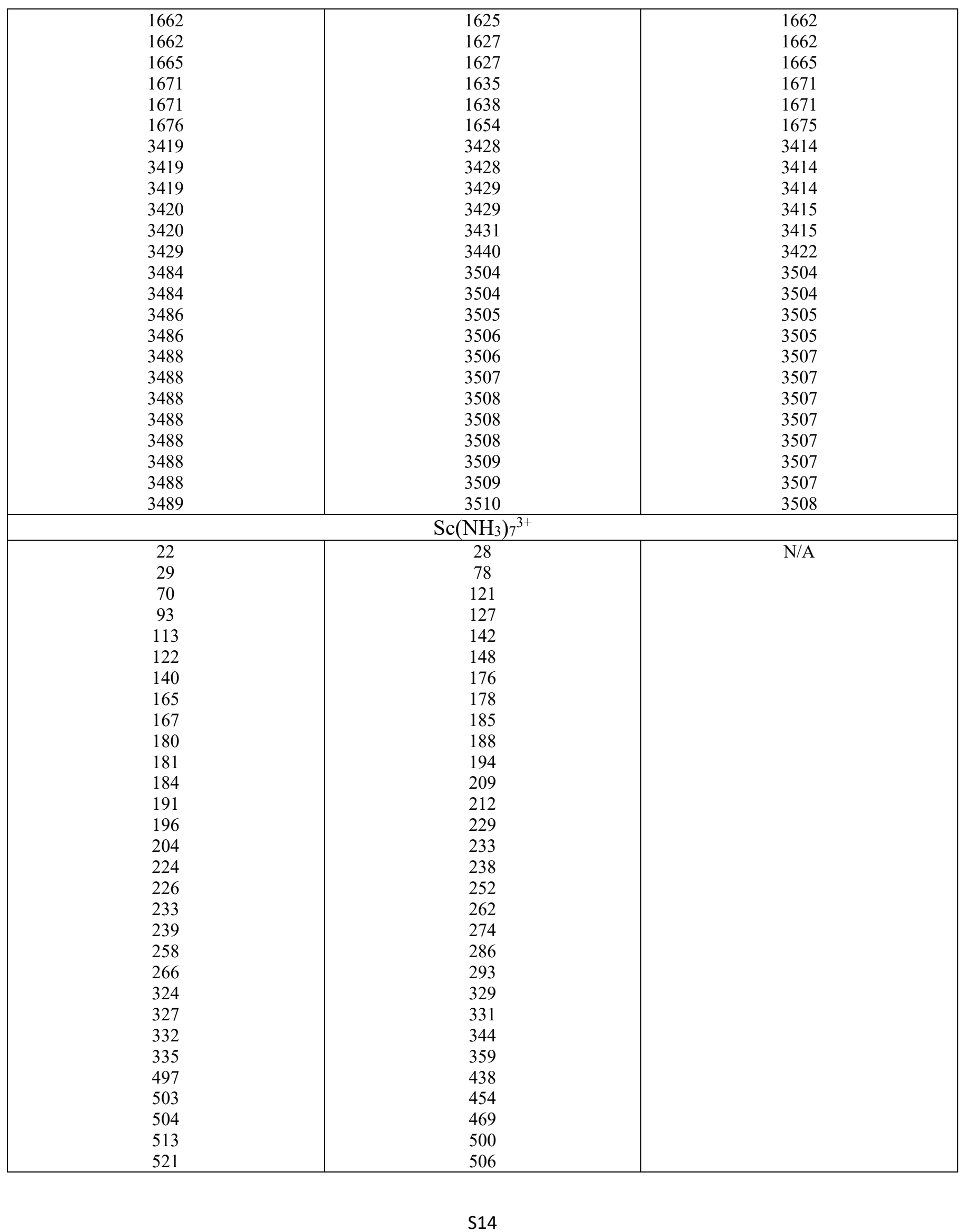




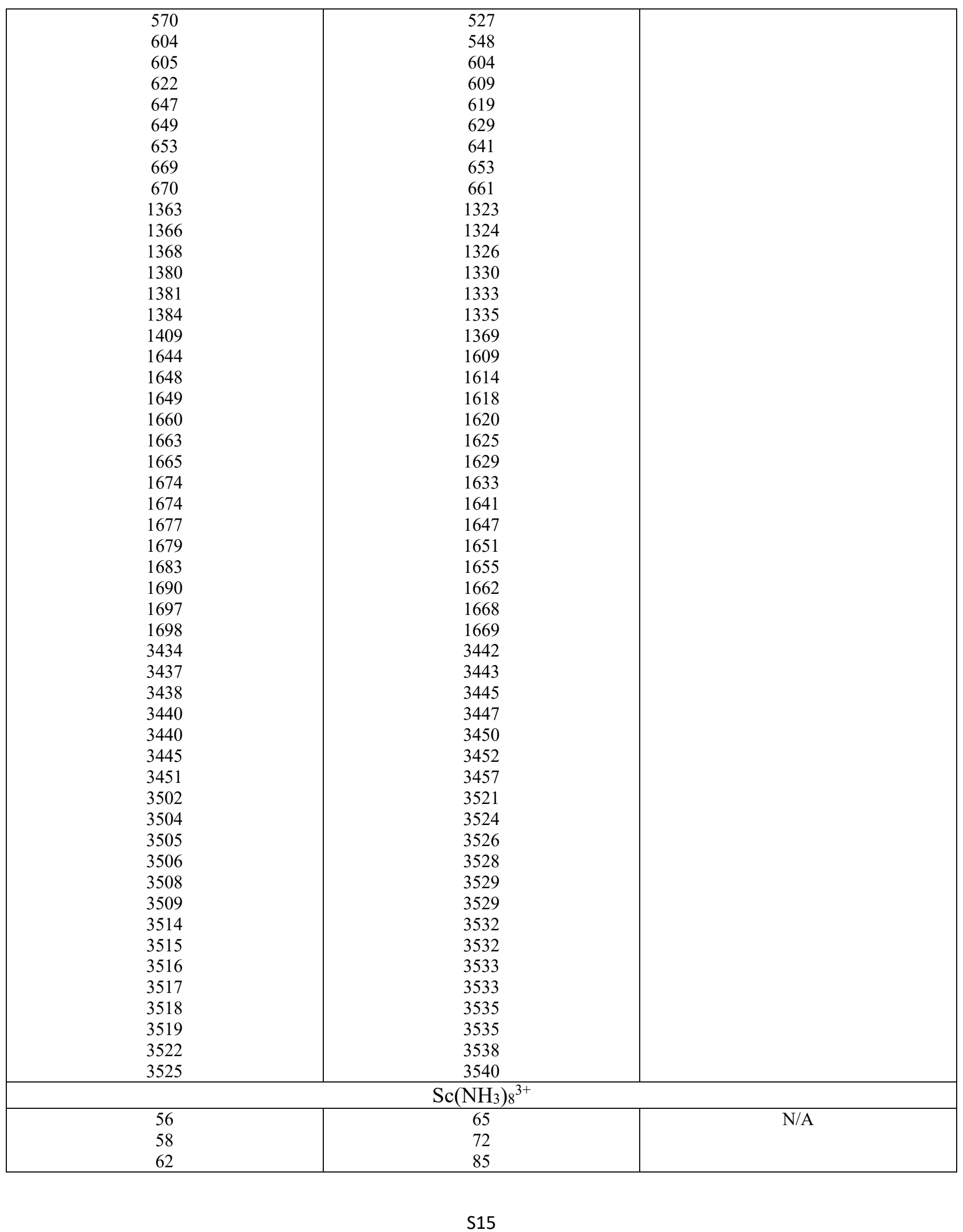




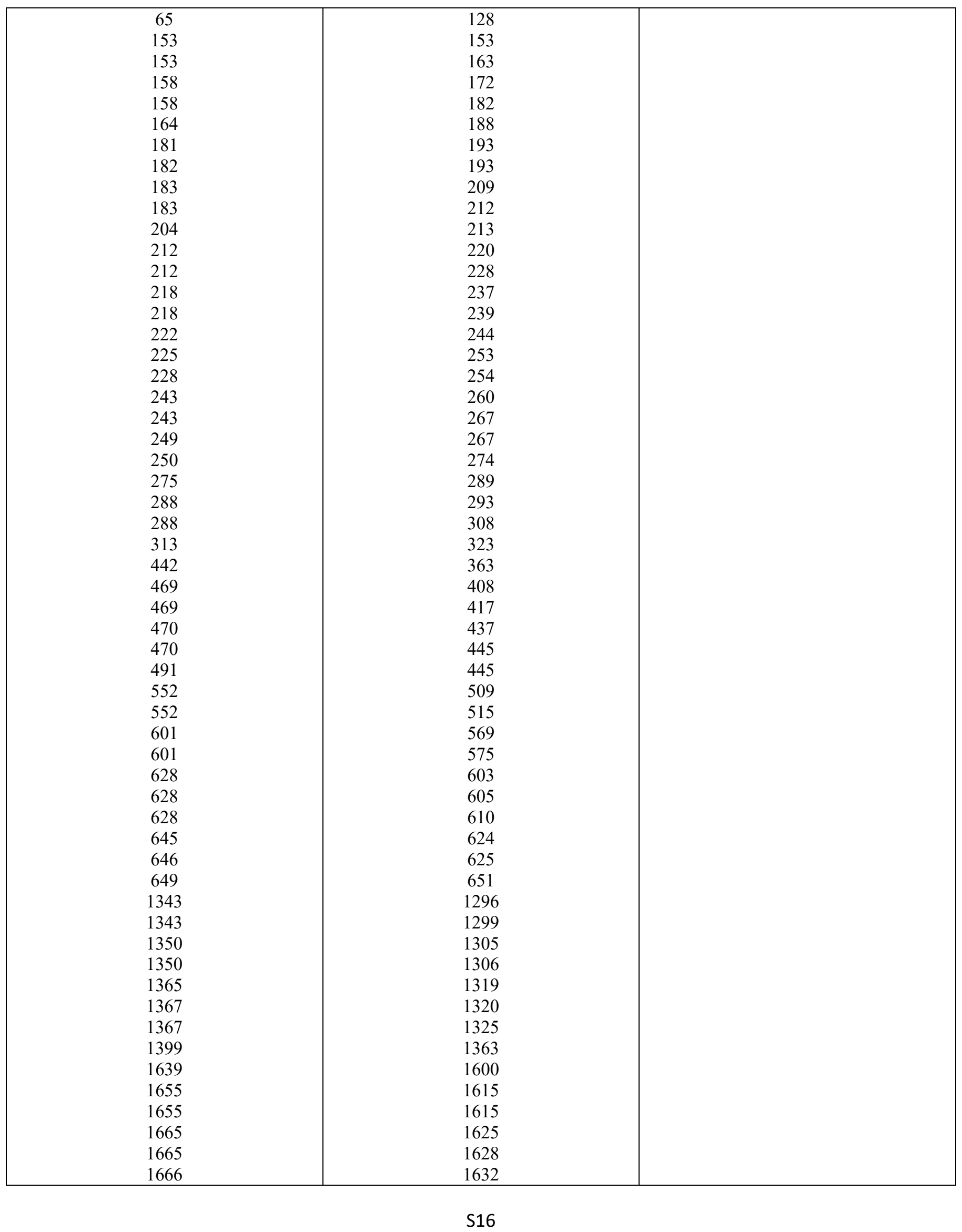




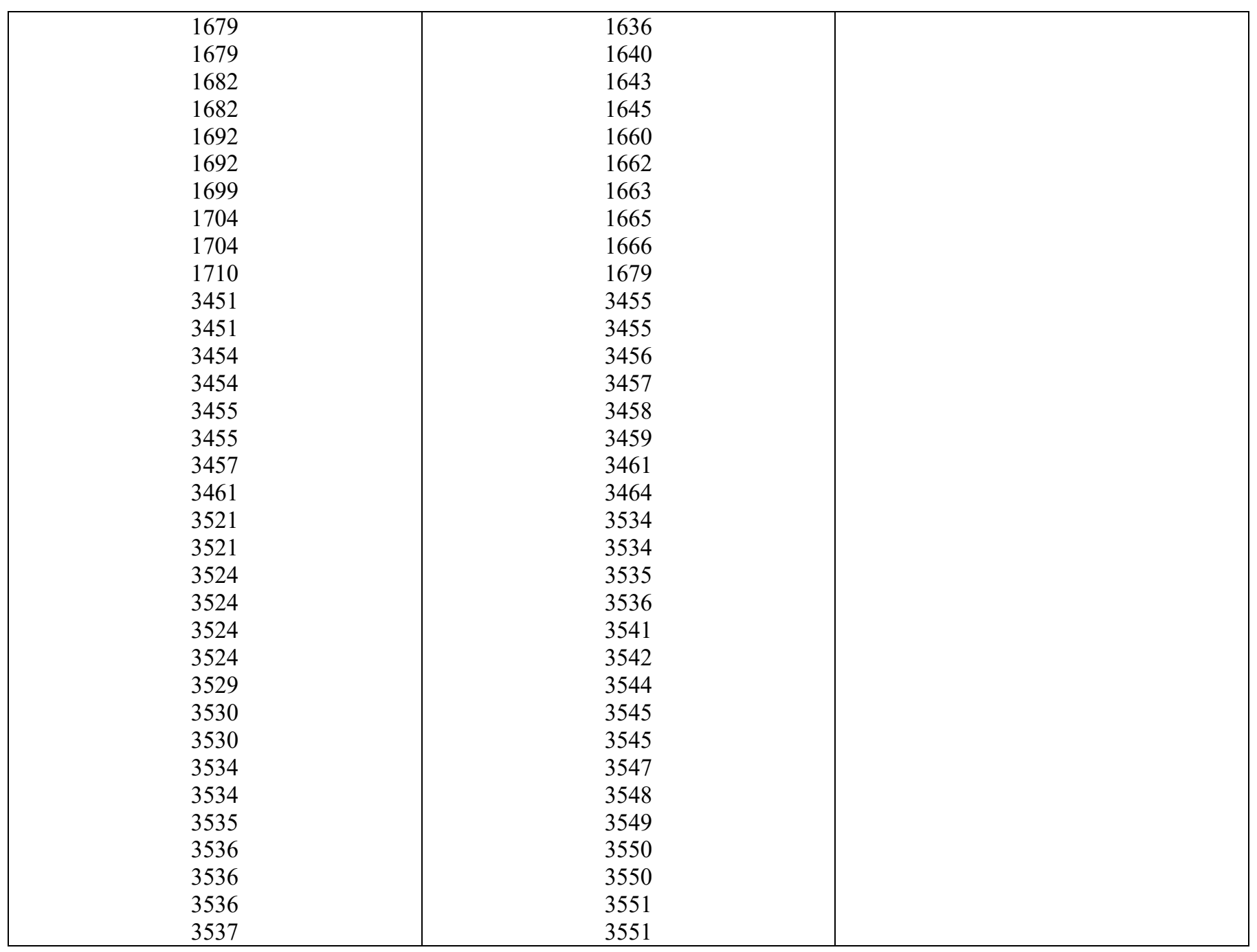


Table S6: Cartesian coordinates $(\AA)$ for the $\mathrm{Sc}\left(\mathrm{NH}_{3}\right)_{\mathrm{n}=1-6^{3+}}$ species optimized at C-MP2 combined with the cc-pwCVTZ(Sc)/cc-pVTZ(N)/aug-cc-pVTZ(H) basis sets.

\begin{tabular}{|c|c|c|c|c|c|c|c|c|c|c|c|}
\hline \multicolumn{4}{|c|}{$\mathrm{Sc}\left(\mathrm{NH}_{3}\right)_{1}{ }^{3+}$} & \multicolumn{4}{|c|}{$\mathrm{Sc}\left(\mathrm{NH}_{3}\right)_{2}{ }^{3+}$} & \multicolumn{4}{|c|}{$\mathrm{Sc}\left(\mathrm{NH}_{3}\right) 3^{3+}$} \\
\hline $\mathrm{N}$ & 1.362098 & 0.000000 & -0.000011 & $\mathrm{~N}$ & 1.784565 & 0.562871 & 0.000079 & $\mathrm{H}$ & 0.613516 & 2.258751 & 1.173549 \\
\hline $\mathrm{H}$ & 1.794617 & -0.828017 & -0.477653 & $\mathrm{H}$ & 2.656041 & -0.002710 & -0.000025 & $\mathrm{~N}$ & 0.781464 & 1.963191 & 0.199249 \\
\hline $\mathrm{H}$ & 1.794625 & 0.827710 & -0.478180 & $\mathrm{H}$ & 1.904025 & 1.192058 & -0.816157 & $\mathrm{H}$ & 1.802249 & 2.069017 & 0.088119 \\
\hline $\mathrm{H}$ & 1.794636 & 0.000303 & 0.955890 & $\mathrm{H}$ & 1.904060 & 1.191811 & 0.816500 & $\mathrm{H}$ & 0.399678 & 2.744711 & -0.357623 \\
\hline \multirow[t]{9}{*}{$\mathrm{Sc}$} & -0.710408 & 0.000000 & 0.000001 & $\mathrm{~N}$ & -1.783933 & 0.563381 & 0.000028 & $\mathrm{~N}$ & -2.090927 & -0.304844 & 0.199257 \\
\hline & & & & $\mathrm{H}$ & -2.405473 & 0.394951 & 0.815022 & $\mathrm{H}$ & -2.262901 & -0.598200 & 1.173521 \\
\hline & & & & $\mathrm{H}$ & -1.652579 & 1.591453 & 0.000322 & $\mathrm{H}$ & -2.692952 & 0.526296 & 0.088253 \\
\hline & & & & $\mathrm{H}$ & -2.405271 & 0.395390 & -0.815211 & $\mathrm{H}$ & -2.576877 & -1.026154 & -0.357702 \\
\hline & & & & $\mathrm{Sc}$ & -0.000249 & -0.602224 & -0.000057 & $\mathrm{~N}$ & 1.309488 & -1.658336 & 0.199251 \\
\hline & & & & & & & & $\mathrm{H}$ & 1.649441 & -1.660643 & 1.173545 \\
\hline & & & & & & & & $\mathrm{H}$ & 0.890776 & -2.595291 & 0.088143 \\
\hline & & & & & & & & $\mathrm{H}$ & 2.177188 & -1.718436 & -0.357635 \\
\hline & & & & & & & & $\mathrm{Sc}$ & -0.000014 & -0.000006 & 8403 \\
\hline \multicolumn{4}{|c|}{$\mathrm{Sc}\left(\mathrm{NH}_{3}\right) 4^{3+}$} & \multicolumn{4}{|c|}{$\mathrm{Sc}\left(\mathrm{NH}_{3}\right) 5^{3+}$} & \multicolumn{4}{|c|}{$\mathrm{Sc}\left(\mathrm{NH}_{3}\right)_{6}{ }^{3+}$} \\
\hline $\mathrm{Sc}$ & 0.000017 & 0.000062 & -0.000058 & $\mathrm{~N}$ & 0.003801 & -1.743800 & 358 & $\mathrm{Sc}$ & -0.000008 & -0.000007 & 012 \\
\hline $\mathrm{N}$ & 1.583893 & 1.013733 & -1.164075 & $\mathrm{H}$ & -0.805425 & -1.800265 & -2.0 & $\mathrm{~N}$ & 1838 & -1.028199 & 2660 \\
\hline $\mathrm{H}$ & 1.245796 & 1.683423 & -1.86 & $\mathrm{H}$ & 0.80 & -1.784912 & -2.0 & $\mathrm{H}$ & 253 & -1.889979 & 432 \\
\hline $\mathrm{H}$ & 2.194586 & 0.387527 & -1.70 & $\mathrm{H}$ & 0.016474 & -2.663507 & -0.5 & $\mathrm{H}$ & 507 & -1.309091 & 987 \\
\hline $\mathrm{N}$ & 0.926556 & -1.382927 & 1.455950 & $\mathrm{~N}$ & 2.280744 & 0.002836 & 0.0 & $\mathrm{~N}$ & 1.336091 & -0.832782 & 580 \\
\hline $\mathrm{H}$ & 1.555259 & -0.939257 & 2.138542 & $\mathrm{H}$ & 2.717838 & -0.894333 & 0.234613 & $\mathrm{H}$ & 0.955381 & -0.752038 & 2.625784 \\
\hline $\mathrm{H}$ & 1.508351 & -2.123776 & 1.042788 & $\mathrm{H}$ & 2.716782 & 0.249707 & -0.893534 & $\mathrm{H}$ & 2.259819 & -0.400847 & 1.760824 \\
\hline $\mathrm{N}$ & -1.202786 & 1.509818 & 1.079296 & $\mathrm{H}$ & 2.715805 & 0.654714 & 0.661339 & $\mathrm{~N}$ & -1.342434 & 1.188566 & 1.443913 \\
\hline $\mathrm{H}$ & -1.673588 & 2.200710 & 0.480081 & $\mathrm{~N}$ & -2.280931 & -0.002396 & -0.001623 & $\mathrm{H}$ & -0.964714 & 1.324297 & 2.385011 \\
\hline $\mathrm{H}$ & -0.676168 & 2.091221 & 1.744647 & $\mathrm{H}$ & -2.716236 & -0.900427 & & $\mathrm{H}$ & 6031 & 4675 & 920 \\
\hline $\mathrm{N}$ & -1.307690 & -1.140725 & -1.3 & $\mathrm{H}$ & -2.718123 & 0.648817 & & $\mathrm{~N}$ & 495 & 385 & 814 \\
\hline $\mathrm{H}$ & -2.080331 & -1.645136 & -0.916482 & $\mathrm{H}$ & -2.716612 & 0.243105 & -0.89 & $\mathrm{H}$ & -2.261228 & 0.998135 & 2949 \\
\hline $\mathrm{H}$ & -1.781217 & -0.577546 & -2.089839 & $\mathrm{Sc}$ & -0.000095 & 0.000034 & -0.000048 & $\mathrm{H}$ & -0.956386 & 1.397862 & -2.345968 \\
\hline $\mathrm{H}$ & 0.263047 & -1.902347 & 2.045870 & $\mathrm{~N}$ & -0.002614 & 2.099066 & -0.800702 & $\mathrm{H}$ & 1.547784 & -1.830376 & 1.597741 \\
\hline $\mathrm{H}$ & 2.246322 & 1.568693 & -0.606175 & $\mathrm{H}$ & -0.007383 & 2.165136 & -1.824981 & $\mathrm{H}$ & 1.549530 & -0.456468 & -2.385273 \\
\hline $\mathrm{H}$ & -0.833527 & -1.872812 & -1.916275 & $\mathrm{H}$ & -0.805400 & 2.672239 & -0.5 & $\mathrm{H}$ & -1.539424 & -0.093144 & -2.433134 \\
\hline \multirow[t]{9}{*}{$\mathrm{H}$} & -1.968696 & 1.128693 & 1.650407 & $\mathrm{H}$ & 0.803253 & 2.671497 & -0.529553 & $\mathrm{H}$ & -1.554754 & 2.141540 & 1.138194 \\
\hline & & & & $\mathrm{N}$ & -0.000858 & -0.355768 & 2.21 & $\mathrm{~N}$ & 6697 & 1.878081 & 452 \\
\hline & & & & $\mathrm{H}$ & -0.804462 & -0.881703 & 2.576216 & $\mathrm{H}$ & 1.533783 & 2.304861 & 0.796015 \\
\hline & & & & $\mathrm{H}$ & 0.804215 & -0.878697 & 2.577305 & $\mathrm{H}$ & 0.943234 & 2.656462 & -0.652520 \\
\hline & & & & $\mathrm{H}$ & -0.002828 & 0.498346 & 2.787313 & $\mathrm{H}$ & 2.252250 & 1.738420 & -0.523108 \\
\hline & & & & & & & & $\mathrm{N}$ & -1.327684 & -1.855041 & 0.309410 \\
\hline & & & & & & & & $\mathrm{H}$ & -2.252345 & -1.810859 & -0.125893 \\
\hline & & & & & & & & $\mathrm{H}$ & -1.536806 & -2.068385 & 1.287909 \\
\hline & & & & & & & & $\mathrm{H}$ & -0.943777 & -2.734995 & -0.044226 \\
\hline
\end{tabular}


Table S7: Electronic energies (a.u.) for the $\mathrm{Sc}\left(\mathrm{NH}_{3}\right)_{\mathrm{n}=1-8}{ }^{2+}$ species optimized at CAM-B3LYP, MN15, MP2 and C-MP2 combined with the cc-pVTZ $(\mathrm{Sc}, \mathrm{N}) /$ aug-cc-pVTZ $(\mathrm{H})$ basis sets for the former three methods and cc-pwCVTZ(Sc)/cc-pVTZ(N)/aug-cc-pVTZ(H) for C-MP2.

\begin{tabular}{crrcr}
\hline$n$ & CAM-B3LYP & MN15 & MP2 & C-MP2 \\
\hline 0 & -759.940335 & -759.889108 & -759.095823 & -759.379776 \\
1 & -816.630455 & -816.527405 & -815.671102 & -815.964143 \\
2 & -873.293479 & -873.142118 & -872.227158 & -872.526070 \\
3 & -929.940935 & -929.741986 & -928.767940 & -929.073913 \\
4 & -986.566546 & -986.325863 & -985.293216 & -985.602354 \\
5 & -1043.170131 & -1042.891076 & -1041.799398 & -1042.109983 \\
6 & -1099.770126 & -1099.455558 & -1098.303007 & \\
$6^{a}$ & -1099.770118 & -1099.455066 & -1098.303009 & \\
7 & -1156.342422 & -1155.994792 & -1154.781187 & \\
8 & -1212.914384 & -1212.533863 & -1211.260207 & \\
$8^{a}$ & -1212.914262 & -1212.532856 & -1211.260168 & \\
\hline
\end{tabular}

${ }^{a}$ Energies of the imposed $\mathrm{C}_{2 \mathrm{~h}}$ or $\mathrm{C}_{2 \mathrm{v}}$ structures for $n=6$ or 8 .

Table S8: Cartesian coordinates $(\AA)$ for the $\mathrm{Sc}\left(\mathrm{NH}_{3}\right)_{n=6,8}{ }^{2+}$ species optimized at CAM-B3LYP, MN15, and MP2 with the cc-pVTZ $(\mathrm{Sc}, \mathrm{N})$ aug-cc-pVTZ $(\mathrm{H})$ basis set constrained in $\mathrm{C}_{2 h}(n=6)$ and $\mathrm{C}_{2 v}(n=8)$ symmetries used for the excitation energy calculations.

\begin{tabular}{|c|c|c|c|c|c|c|c|c|c|c|c|}
\hline \multicolumn{12}{|c|}{$\mathrm{Sc}\left(\mathrm{NH}_{3}\right)_{6}{ }^{2+}$} \\
\hline \multicolumn{4}{|c|}{ MN15 } & \multicolumn{4}{|c|}{ CAM-B3LYP } & \multicolumn{4}{|c|}{ MP2 } \\
\hline $\mathrm{Sc}$ & 0.000000 & 0.000000 & 0.000000 & $\mathrm{Sc}$ & 0.000000 & 0.000000 & 0.000000 & $\mathrm{Sc}$ & 0.000000 & 0.000000 & 0.000000 \\
\hline $\mathrm{N}$ & -2.385535 & 0.237026 & 0.000000 & $\mathrm{~N}$ & -2.413363 & 0.270349 & 0.000000 & $\mathrm{~N}$ & -2.445167 & 0.277178 & -0.000000 \\
\hline $\mathrm{H}$ & -2.807973 & -0.219806 & 0.806475 & $\mathrm{H}$ & -2.860488 & -0.164332 & 0.803237 & $\mathrm{H}$ & -2.904174 & -0.151352 & 0.800521 \\
\hline $\mathrm{H}$ & -2.807973 & -0.219806 & -0.806475 & $\mathrm{H}$ & -2.860488 & -0.164332 & -0.803237 & $\mathrm{H}$ & -2.904174 & -0.151352 & -0.800521 \\
\hline $\mathrm{N}$ & -0.000000 & -1.638286 & -1.751010 & $\mathrm{~N}$ & -0.000000 & -1.651115 & -1.780689 & $\mathrm{~N}$ & -0.000000 & -1.672292 & -1.805338 \\
\hline $\mathrm{H}$ & 0.661234 & -1.501100 & -2.514325 & $\mathrm{H}$ & 0.669830 & -1.495192 & -2.530199 & $\mathrm{H}$ & 0.671485 & -1.520304 & -2.554547 \\
\hline $\mathrm{H}$ & -0.914472 & -1.712716 & -2.193456 & $\mathrm{H}$ & -0.900009 & -1.730052 & -2.247202 & $\mathrm{H}$ & -0.894041 & -1.755930 & -2.283725 \\
\hline $\mathrm{N}$ & 2.385535 & -0.237026 & 0.000000 & $\mathrm{~N}$ & 2.413363 & -0.270349 & 0.000000 & $\mathrm{~N}$ & 2.445167 & -0.277178 & -0.000000 \\
\hline $\mathrm{H}$ & 2.756138 & -1.186427 & -0.000000 & $\mathrm{H}$ & 2.751966 & -1.229436 & -0.000000 & $\mathrm{H}$ & 2.785659 & -1.236016 & 0.000000 \\
\hline $\mathrm{H}$ & 2.807973 & 0.219806 & 0.806475 & $\mathrm{H}$ & 2.860488 & 0.164332 & 0.803237 & $\mathrm{H}$ & 2.904174 & 0.151352 & 0.800521 \\
\hline $\mathrm{N}$ & 0.000000 & 1.638286 & 1.751010 & $\mathrm{~N}$ & 0.000000 & 1.651115 & 1.780689 & $\mathrm{~N}$ & 0.000000 & 1.672292 & 1.805338 \\
\hline $\mathrm{H}$ & 0.914472 & 1.712716 & 2.193456 & $\mathrm{H}$ & 0.900009 & 1.730052 & 2.247202 & $\mathrm{H}$ & 0.894041 & 1.755930 & 2.283725 \\
\hline $\mathrm{H}$ & -0.189523 & 2.572306 & 1.391504 & $\mathrm{H}$ & -0.195038 & 2.591979 & 1.448080 & $\mathrm{H}$ & -0.192677 & 2.618481 & 1.484773 \\
\hline $\mathrm{H}$ & 0.189523 & -2.572306 & -1.391504 & $\mathrm{H}$ & 0.195038 & -2.591979 & -1.448080 & $\mathrm{H}$ & 0.192677 & -2.618481 & -1.484773 \\
\hline $\mathrm{H}$ & -2.756138 & 1.186427 & -0.000000 & $\mathrm{H}$ & -2.751966 & 1.229436 & -0.000000 & $\mathrm{H}$ & -2.785659 & 1.236016 & 0.000000 \\
\hline $\mathrm{H}$ & -0.661234 & 1.501100 & 2.514325 & $\mathrm{H}$ & -0.669830 & 1.495192 & 2.530199 & $\mathrm{H}$ & -0.671485 & 1.520304 & 2.554547 \\
\hline $\mathrm{H}$ & 2.807973 & 0.219806 & -0.806475 & $\mathrm{H}$ & 2.860488 & 0.164332 & -0.803237 & $\mathrm{H}$ & 2.904174 & 0.151352 & -0.800521 \\
\hline
\end{tabular}




\begin{tabular}{|c|c|c|c|c|c|c|c|c|c|c|c|}
\hline & & & & & & & & & & & \\
\hline $\mathrm{N}$ & 0.000000 & 1.638286 & -1.751010 & $\mathrm{~N}$ & 0.000000 & 1.651115 & -1.780689 & $\mathrm{~N}$ & 0.000000 & 1.672292 & -1.805338 \\
\hline $\mathrm{H}$ & 0.914472 & 1.712716 & -2.193456 & $\mathrm{H}$ & 0.900009 & 1.730052 & -2.247202 & $\mathrm{H}$ & 0.894041 & 1.755930 & -2.283725 \\
\hline $\mathrm{H}$ & -0.189523 & 2.572306 & -1.391504 & $\mathrm{H}$ & -0.195038 & 2.591979 & -1.448080 & $\mathrm{H}$ & -0.192677 & 2.618481 & -1.484773 \\
\hline $\mathrm{H}$ & -0.661234 & 1.501100 & -2.514325 & $\mathrm{H}$ & -0.669830 & 1.495192 & -2.530199 & $\mathrm{H}$ & -0.671485 & 1.520304 & -2.554547 \\
\hline $\mathrm{N}$ & -0.000000 & -1.638286 & 1.751010 & $\mathrm{~N}$ & -0.000000 & -1.651115 & 1.780689 & $\mathrm{~N}$ & -0.000000 & -1.672292 & 1.805338 \\
\hline $\mathrm{H}$ & 0.661234 & -1.501100 & 2.514325 & $\mathrm{H}$ & 0.669830 & -1.495192 & 2.530199 & $\mathrm{H}$ & 0.671485 & -1.520304 & 2.554547 \\
\hline $\mathrm{H}$ & 0.189523 & -2.572306 & 1.391504 & $\mathrm{H}$ & 0.195038 & -2.591979 & 1.448080 & $\mathrm{H}$ & 0.192677 & -2.618481 & 1.484773 \\
\hline $\mathrm{H}$ & -0.914472 & -1.712716 & 2.193456 & $\mathrm{H}$ & -0.900009 & -1.730052 & 2.247202 & $\mathrm{H}$ & -0.8 & -1.755930 & 2.283725 \\
\hline \multicolumn{12}{|c|}{$\mathrm{Sc}\left(\mathrm{NH}_{3}\right) 8^{2+}$} \\
\hline \multicolumn{4}{|c|}{ MN15 } & \multicolumn{4}{|c|}{ CAM-B3LYP } & \multicolumn{4}{|c|}{ MP2 } \\
\hline $\mathrm{H}$ & 0.8 & 2.712042 & 1.2 & $\overline{\mathrm{H}}$ & 5003 & 2.752043 & 1 & $\mathrm{H}$ & 77 & 2.772916 & 56 \\
\hline $\mathrm{H}$ & -0.810886 & 2.712042 & 1.239274 & $\mathrm{H}$ & -0.805003 & 2.752043 & 1.246171 & $\mathrm{H}$ & -0.802077 & 2.772916 & 1.265156 \\
\hline $\mathrm{N}$ & 0.000000 & 2.109014 & 1.358782 & $\mathrm{~N}$ & 0.000000 & 2.149008 & 1.385785 & $\mathrm{~N}$ & 0.000000 & 2.163314 & 1.400577 \\
\hline $\mathrm{H}$ & 2.712042 & 0.810886 & 1.239274 & $\mathrm{H}$ & 2.752043 & 0.805003 & 1.246171 & $\mathrm{H}$ & 2.772916 & 0.802077 & 1.265156 \\
\hline $\mathrm{H}$ & 0.000000 & 1.844302 & 2.341007 & $\mathrm{H}$ & 0.000000 & 1.941913 & 2.379655 & $\mathrm{H}$ & 0.000000 & 1.971954 & 2.398871 \\
\hline $\mathrm{N}$ & 2.109014 & -0.000000 & 1.358782 & $\mathrm{~N}$ & 2.149008 & -0.000000 & 1.385785 & $\mathrm{~N}$ & 2.163314 & -0.000000 & 1.400577 \\
\hline $\mathrm{H}$ & 1.844302 & -0.000000 & 2.341007 & $\mathrm{H}$ & 1.941913 & -0.000000 & 2.379655 & $\mathrm{H}$ & 1.971954 & -0.000000 & 2.398871 \\
\hline $\mathrm{H}$ & 2.712042 & -0.810886 & 1.239274 & $\mathrm{H}$ & 2.752043 & -0.805003 & 1.246171 & $\mathrm{H}$ & 2.772916 & -0.802077 & 1.265156 \\
\hline $\mathrm{Sc}$ & 0.000000 & 0.000000 & 0.000000 & $\mathrm{Sc}$ & 0.000000 & 0.000000 & 0.000000 & $\mathrm{Sc}$ & 0.000000 & 0.000000 & 0.000000 \\
\hline $\mathrm{H}$ & -2.712042 & 0.810886 & 1.239274 & $\mathrm{H}$ & -2.752043 & 0.805003 & 1.246171 & $\mathrm{H}$ & -2.772916 & 0.802077 & 1.265156 \\
\hline $\mathrm{N}$ & -2.109014 & 0.000000 & 1.3 & $\mathrm{~N}$ & -2.149008 & 0.000000 & 1.38 & $\mathrm{~N}$ & -2.1 & 0.000000 & 1.400577 \\
\hline $\mathrm{H}$ & -1.844302 & 0.000000 & 2.341007 & $\mathrm{H}$ & -1.941913 & 0.000000 & 2.379655 & $\mathrm{H}$ & -1.971954 & 0.000000 & 2.398871 \\
\hline $\mathrm{H}$ & -0.000000 & -1.844302 & 2.341007 & $\mathrm{H}$ & -0.000000 & -1.941913 & 2.379655 & $\mathrm{H}$ & -0.000000 & -1.971954 & 2.398871 \\
\hline $\mathrm{N}$ & -0.000000 & -2.109014 & 1.358782 & $\mathrm{~N}$ & -0.000000 & -2.149008 & 1.385785 & $\mathrm{~N}$ & -0.000000 & -2.163314 & 1.400577 \\
\hline $\mathrm{H}$ & -2.712042 & -0.810886 & 1.239274 & $\mathrm{H}$ & -2.752043 & -0.805003 & 1.246171 & $\mathrm{H}$ & -2.772916 & -0.802077 & 1.265156 \\
\hline $\mathrm{H}$ & 0.810886 & -2.712042 & 1.239274 & $\mathrm{H}$ & 0.805003 & -2.752043 & 1.246171 & $\mathrm{H}$ & 0.802077 & -2.772916 & 1.265156 \\
\hline $\mathrm{H}$ & -0.810886 & -2.712042 & 1.239274 & $\mathrm{H}$ & -0.805003 & -2.7 & 1.246171 & $\mathrm{H}$ & -0.802077 & -2.772916 & 5156 \\
\hline $\mathrm{H}$ & 1.34 & 2.491087 & -1.23 & $\mathrm{H}$ & 66 & 2.5 & -1.24 & $\mathrm{H}$ & 1.3 & 902 & -1.2 \\
\hline $\mathrm{H}$ & 2.491087 & 1.344320 & -1.239274 & $\mathrm{H}$ & 2.515211 & 1.376766 & -1.246171 & $\mathrm{H}$ & 2.527902 & 1.393594 & -1.265156 \\
\hline $\mathrm{N}$ & 1.491298 & 1.491298 & -1.358782 & $\mathrm{~N}$ & 1.519578 & 1.519578 & -1.385785 & $\mathrm{~N}$ & 1.529694 & 1.529694 & -1.400577 \\
\hline $\mathrm{H}$ & -1.344320 & 2.491087 & -1.239274 & $\mathrm{H}$ & -1.376766 & 2.515211 & -1.246171 & $\mathrm{H}$ & -1.393594 & 2.527902 & -1.265156 \\
\hline $\mathrm{H}$ & 1.304119 & 1.304119 & -2.341007 & $\mathrm{H}$ & 1.373140 & 1.373140 & -2.379655 & $\mathrm{H}$ & 1.394382 & 1.394382 & -2.398871 \\
\hline $\mathrm{N}$ & -1.491298 & 1.491298 & -1.358782 & $\mathrm{~N}$ & -1.519578 & 1.519578 & -1.385785 & $\mathrm{~N}$ & -1.529694 & 1.529694 & -1.400577 \\
\hline $\mathrm{H}$ & -1.304119 & 1.304119 & -2.341007 & $\mathrm{H}$ & -1.373140 & 1.373140 & -2.379655 & $\mathrm{H}$ & -1.394382 & 1.394382 & -2.398871 \\
\hline $\mathrm{H}$ & -2.491087 & 1.344320 & -1.239274 & $\mathrm{H}$ & -2.515211 & 1.376766 & -1.246171 & $\mathrm{H}$ & -2.527902 & 1.393594 & -1.265156 \\
\hline $\mathrm{H}$ & 2.491087 & -1.344320 & -1.239274 & $\mathrm{H}$ & 2.515211 & -1.376766 & -1.246171 & $\mathrm{H}$ & 2.527902 & -1.393594 & -1.265156 \\
\hline $\mathrm{N}$ & 1.491298 & -1.491298 & -1.358782 & $\mathrm{~N}$ & 1.519578 & -1.519578 & -1.385785 & $\mathrm{~N}$ & 1.529694 & -1.529694 & -1.400577 \\
\hline $\mathrm{H}$ & 1.304119 & -1.304119 & -2.341007 & $\mathrm{H}$ & 1.373140 & -1.373140 & -2.379655 & $\mathrm{H}$ & 1.394382 & -1.394382 & -2.398871 \\
\hline $\mathrm{H}$ & -1.304119 & -1.304119 & -2.341007 & $\mathrm{H}$ & -1.373140 & -1.373140 & -2.379655 & $\mathrm{H}$ & -1.394382 & -1.394382 & -2.398871 \\
\hline $\mathrm{N}$ & -1.491298 & -1.491298 & -1.358782 & $\mathrm{~N}$ & -1.519578 & -1.519578 & -1.385785 & $\mathrm{~N}$ & -1.529694 & -1.529694 & -1.400577 \\
\hline $\mathrm{H}$ & 1.344320 & -2.491087 & -1.239274 & $\mathrm{H}$ & 1.376766 & -2.515211 & -1.246171 & $\mathrm{H}$ & 1.393594 & -2.527902 & -1.265156 \\
\hline $\mathrm{H}$ & -2.491087 & -1.344320 & -1.239274 & $\mathrm{H}$ & -2.515211 & -1.376766 & -1.246171 & $\mathrm{H}$ & -2.527902 & -1.393594 & -1.265156 \\
\hline $\mathrm{H}$ & -1.344320 & -2.491087 & -1.239274 & $\mathrm{H}$ & -1.376766 & -2.515211 & -1.246171 & $\mathrm{H}$ & -1.393594 & -2.527902 & -1.265156 \\
\hline
\end{tabular}


Table S9: Cartesian coordinates $(\AA)$ for the $\mathrm{Sc}\left(\mathrm{NH}_{3}\right)_{\mathrm{n}=1-8}{ }^{2+}$ species optimized at CAM-B3LYP, MN15, and MP2 combined with the cc-pVTZ $(\mathrm{Sc}, \mathrm{N})$ aug-cc-pVTZ $(\mathrm{H})$ basis sets.

\begin{tabular}{|c|c|c|c|c|c|c|c|c|c|c|c|}
\hline \multicolumn{4}{|c|}{ CAM-B3LYP } & \multicolumn{4}{|c|}{ MN15 } & \multicolumn{4}{|c|}{ MP2 } \\
\hline \multicolumn{12}{|c|}{$\mathrm{Sc}\left(\mathrm{NH}_{3}\right)_{1}{ }^{2+}$} \\
\hline $\mathrm{N}$ & -1.467076 & -0.000058 & 0.000032 & $\mathrm{~N}$ & 0872 & -0.000078 & 0.00 & $\mathrm{~N}$ & 1.513738 & -0.000063 & 0.000008 \\
\hline $\mathrm{H}$ & -1.885770 & 0.897657 & -0.268537 & $\mathrm{H}$ & -1.891300 & 0.936847 & -0.045781 & $\mathrm{H}$ & -1.940673 & 0.930188 & -0.042108 \\
\hline $\mathrm{H}$ & -1.887118 & -0.680834 & -0.642996 & $\mathrm{H}$ & -1.893313 & -0.507423 & -0.788018 & $\mathrm{H}$ & -1.942010 & -0.501104 & -0.784223 \\
\hline $\mathrm{H}$ & -1.887959 & -0.215957 & 0.910916 & $\mathrm{H}$ & -1.893523 & -0.428220 & 0.833610 & $\mathrm{H}$ & -1.942167 & -0.428228 & 0.826215 \\
\hline $\mathrm{Sc}$ & 0.758589 & -0.000022 & 0.000019 & $\mathrm{Sc}$ & 0.760678 & -0.000031 & 0.000004 & $\mathrm{Sc}$ & 0.781953 & -0.000020 & 0003 \\
\hline \multicolumn{12}{|c|}{$\mathrm{Sc}\left(\mathrm{NH}_{3}\right)_{2}{ }^{2+}$} \\
\hline $\mathrm{N}$ & -2.123403 & -0.404920 & -0.000003 & $\mathrm{~N}$ & -2.104397 & -0.425862 & 0.000004 & $\mathrm{~N}$ & -2.282151 & -0.266309 & 0.000001 \\
\hline $\mathrm{H}$ & -2.686777 & -0.129254 & 0.808781 & $\mathrm{H}$ & -2.677407 & -0.165589 & 0.809565 & $\mathrm{H}$ & -2.808135 & 0.081748 & 0.804667 \\
\hline $\mathrm{H}$ & -2.687452 & -0.127787 & -0.807818 & $\mathrm{H}$ & -2.677657 & -0.164859 & -0.809146 & $\mathrm{H}$ & -2.808340 & 0.082608 & -0.804160 \\
\hline $\mathrm{H}$ & -2.162860 & -1.427102 & -0.000926 & $\mathrm{H}$ & -2.117631 & -1.450672 & -0.000457 & $\mathrm{H}$ & -2.472768 & -1.270520 & -0.000512 \\
\hline $\mathrm{N}$ & 2.123409 & -0.404921 & 0.000015 & $\mathrm{~N}$ & 2.104394 & -0.425865 & -0.00 & $\mathrm{~N}$ & 2.282151 & -0.266309 & -0.000000 \\
\hline $\mathrm{H}$ & 2.686840 & -0.129635 & 0.80 & $\mathrm{H}$ & 391 & -0.165169 & & $\mathrm{H}$ & 196 & 0.082036 & 502 \\
\hline $\mathrm{H}$ & 2.162791 & -1.427103 & -0.0 & $\mathrm{H}$ & 682 & -1.450675 & & $\mathrm{H}$ & & -1.270519 & 69 \\
\hline $\mathrm{H}$ & 2.687406 & -0.127435 & -0.80 & $\mathrm{H}$ & 2.677651 & -0.165240 & -0.8 & $\mathrm{H}$ & 277 & 0.082322 & -0 . \\
\hline $\mathrm{Sc}$ & 0.000000 & 0.430343 & 0.000003 & $\mathrm{Sc}$ & -0.000000 & 0.453538 & -0.0 & $\mathrm{Sc}$ & 000 & 0.282888 & \\
\hline \multicolumn{12}{|c|}{$\mathrm{Sc}\left(\mathrm{NH}_{3}\right)_{3}{ }^{2+}$} \\
\hline $\mathrm{H}$ & 1.098631 & 2.464790 & -0.92 & $\mathrm{H}$ & & 2.682382 & -0.9 & $\mathrm{H}$ & & 2.755394 & -0.938128 \\
\hline $\mathrm{N}$ & 1.044508 & 2.035256 & -0.0 & $\mathrm{~N}$ & & 2.283914 & & $\mathrm{~N}$ & & 2.340349 & \\
\hline $\mathrm{H}$ & 0.591559 & 2.736069 & & $\mathrm{H}$ & -0. & 2.712096 & & $\mathrm{H}$ & & 2.77 & \\
\hline $\mathrm{H}$ & 2.010340 & 2.011207 & & $\mathrm{H}$ & 996 & 2.702436 & & $\mathrm{H}$ & 62 & 2.76 & \\
\hline $\mathrm{N}$ & 1.244471 & -1.919819 & -0.0 & $\mathrm{~N}$ & 891 & -1.149951 & -0.0 & $\mathrm{~N}$ & 01 & -1.178495 & 93 \\
\hline $\mathrm{H}$ & 2.005796 & -1.917484 & -0.6 & $\mathrm{H}$ & 936 & -0.690489 & -0 & $\mathrm{H}$ & & -0.728119 & 758 \\
\hline $\mathrm{H}$ & 1.692789 & -2.117708 & 0.895139 & $\mathrm{H}$ & 7217 & -1.295820 & & $\mathrm{H}$ & 288 & -1.325610 & 0.922568 \\
\hline $\mathrm{H}$ & 0.713621 & -2.765671 & -0.217139 & $\mathrm{H}$ & 1.914803 & -2.086772 & -0.420780 & $\mathrm{H}$ & 6263 & -2.115859 & -0.405394 \\
\hline $\mathrm{N}$ & -2.286920 & -0.115113 & -0.0 & $\mathrm{~N}$ & 398 & -1.126545 & $-0 .($ & $\mathrm{N}$ & & -1.155032 & 136 \\
\hline $\mathrm{H}$ & -2.753026 & 0.770695 & -0.2 & $\mathrm{H}$ & & -0.6 & & $\mathrm{H}$ & & -0.6 & 563 \\
\hline $\mathrm{H}$ & -2.66 & -0.766619 & -0. & $\mathrm{H}$ & -1 . & -2.059787 & & $\mathrm{H}$ & & -2.08 & \\
\hline $\mathrm{H}$ & -2.682631 & -0.414871 & 28 & $\mathrm{H}$ & -2.396988 & -1.277235 & & $\mathrm{H}$ & 58 & -1.307205 & 344 \\
\hline $\mathrm{Sc}$ & -0.001193 & -0.000128 & 0.00 & $\mathrm{Sc}$ & -0.000073 & -0.004076 & 217 & $\mathrm{Sc}$ & 0067 & -0.003808 & 0.007049 \\
\hline \multicolumn{12}{|c|}{$\mathrm{Sc}\left(\mathrm{NH}_{3}\right)_{4}{ }^{2+}$} \\
\hline $\mathrm{Sc}$ & -0.000290 & -0.000119 & 01 & $\mathrm{Sc}$ & 0021 & 0.000049 & 02 & $\mathrm{Sc}$ & 016 & 0.000009 & 0.000001 \\
\hline $\mathrm{N}$ & 1.61 & -1.467174 & & $\mathrm{~N}$ & & 236 & & $\mathrm{~N}$ & & 1.588742 & 699 \\
\hline $\mathrm{H}$ & 40 & -1.00 & & $\mathrm{H}$ & -1 . & 2.2 & & $\mathrm{H}$ & & 1.18 & 83 \\
\hline $\mathrm{H}$ & 2.159151 & -1.964446 & 72 & $\mathrm{H}$ & -2.0 & 2.027672 & -0.1 & $\mathrm{H}$ & 32 & 2.107914 & 528 \\
\hline $\mathrm{N}$ & -1.468082 & -1.612806 & -0.825680 & $\mathrm{~N}$ & -1.506334 & -1.557272 & 75 & $\mathrm{~N}$ & -1.588802 & 1.574319 & 740 \\
\hline $\mathrm{H}$ & -2.199822 & -1.220630 & -1.417463 & $\mathrm{H}$ & -1.059298 & -2.255864 & & $\mathrm{H}$ & -2.311123 & 1.160994 & 490 \\
\hline $\mathrm{H}$ & -1.964615 & -2.158847 & -0.121364 & $\mathrm{H}$ & -2.027674 & -2.096174 & 0.147597 & $\mathrm{H}$ & -2.107940 & 2.088638 & 0.099586 \\
\hline $\mathrm{N}$ & -1.612370 & 1.468326 & 0.825871 & $\mathrm{~N}$ & 1.557318 & -1.506376 & -0.842820 & $\mathrm{~N}$ & -1.574379 & -1.588774 & -0.810644 \\
\hline $\mathrm{H}$ & -2.156534 & 1.967038 & & $\mathrm{H}$ & 5629 & -2.028313 & -0.1 & $\mathrm{H}$ & -2.088351 & -2.108208 & 9456 \\
\hline $\mathrm{H}$ & -1.219824 & 2.198299 & & $\mathrm{H}$ & 6418 & -1.059285 & & $\mathrm{H}$ & & -2.310848 & -1.399767 \\
\hline $\mathrm{N}$ & 1.468011 & 1.611771 & & $\mathrm{~N}$ & & 7327 & & $\mathrm{~N}$ & & 03 & 600 \\
\hline $\mathrm{H}$ & 1.009090 & 2.304456 & & $\mathrm{H}$ & & 1.133670 & & $\mathrm{H}$ & 75 & -2.300696 & 977 \\
\hline $\mathrm{H}$ & 1.966634 & 2.156306 & -0 . & $\mathrm{H}$ & 2.028314 & 2.095666 & 0.1 & $\mathrm{H}$ & 201 & -2.088326 & 395 \\
\hline $\mathrm{H}$ & -1.009363 & -2.304129 & -1.418214 & $\mathrm{H}$ & -2.219391 & -1.133581 & & $\mathrm{H}$ & 130 & 2.300468 & 1.399414 \\
\hline $\mathrm{H}$ & 1.221135 & -2.198227 & 1.419078 & $\mathrm{H}$ & -2.256288 & 1.059093 & -1.439457 & $\mathrm{H}$ & 1.161107 & 2.311033 & -1.399528 \\
\hline $\mathrm{H}$ & 2.198077 & 1.219371 & -1.420177 & $\mathrm{H}$ & 1.059534 & 2.256415 & 1.439145 & $\mathrm{H}$ & 2.310977 & -1.161034 & 1.399616 \\
\hline $\mathrm{H}$ & -2.305353 & 009688 & 1.416520 & $\mathrm{H}$ & 1.133657 & -2.218920 & -1.440365 & $\mathrm{H}$ & -2.300813 & -1.182041 & -1.398924 \\
\hline \multicolumn{12}{|c|}{$\mathrm{Sc}\left(\mathrm{NH}_{3}\right) 5^{2+}$} \\
\hline $\mathrm{N}$ & 738 & .531071 & 88 & $\mathrm{~N}$ & 739 & -1.651050 & -1.35 & $\mathrm{~N}$ & -0.783119 & -1.518333 & -1.636362 \\
\hline
\end{tabular}




\begin{tabular}{|c|c|c|c|c|c|c|c|c|c|c|c|}
\hline & & & & & & & & & & & \\
\hline $\mathrm{H}$ & .065979 & 2.090406 & 1.966130 & $\mathrm{H}$ & -0.204037 & -2.362153 & -1.556998 & $\mathrm{H}$ & -0.045832 & -2.095491 & 36297 \\
\hline $\mathrm{H}$ & 1.485876 & -2.209352 & 1.208119 & $\mathrm{H}$ & -1.702037 & -2.161943 & -0.971783 & $\mathrm{H}$ & -1.483340 & -2.187638 & .323018 \\
\hline $\mathrm{H}$ & 1.264501 & -1.090614 & 2.351576 & $\mathrm{H}$ & -1.210563 & -1.323537 & -2.272778 & $\mathrm{H}$ & 208718 & -1.072666 & 47640 \\
\hline $\mathrm{N}$ & 2.316937 & 0.534976 & -0.415615 & $\mathrm{~N}$ & -2.240815 & 0.852462 & 0.204090 & $\mathrm{~N}$ & .356275 & 0.539277 & 0.408255 \\
\hline $\mathrm{H}$ & 2.824476 & 0.982367 & 0.345902 & $\mathrm{H}$ & -2.694401 & 1.250538 & -0.619408 & $\mathrm{H}$ & -2.865308 & 0.988754 & -0.350850 \\
\hline $\mathrm{H}$ & .854653 & -0.303872 & -0.623282 & $\mathrm{H}$ & -2.863548 & 0.103922 & 0.508073 & $\mathrm{H}$ & -2.899850 & -0.298984 & 0.604577 \\
\hline $\mathrm{H}$ & 2.466526 & 1.135745 & -1.222949 & $\mathrm{H}$ & -2.320978 & 1.563422 & 0.930408 & $\mathrm{H}$ & -2.529123 & 1.133898 & 1.216099 \\
\hline $\mathrm{N}$ & -2.364710 & 0.047061 & 0.440583 & $\mathrm{~N}$ & 2.313483 & -0.380136 & -0.435172 & $\mathrm{~N}$ & 2.411645 & 0.003406 & -0.407302 \\
\hline $\mathrm{H}$ & -2.643927 & 0.689479 & 1.178430 & $\mathrm{H}$ & 2.678021 & 0.046862 & -1.286250 & $\mathrm{H}$ & 2.727656 & 0.632548 & -1.142388 \\
\hline $\mathrm{H}$ & -2.968709 & 0.272412 & -0.348040 & $\mathrm{H}$ & 2.960446 & -0.105181 & 0.304832 & $\mathrm{H}$ & 3.011193 & 0.221913 & 0.386434 \\
\hline $\mathrm{H}$ & -2.691090 & -0.861973 & 0.761820 & $\mathrm{H}$ & 2.471529 & -1.379894 & -0.562144 & $\mathrm{H}$ & 2.736420 & -0.911056 & -0.715632 \\
\hline $\mathrm{Sc}$ & -0.004095 & 383 & 0.0 & $\mathrm{Sc}$ & & & -0.061955 & $\mathrm{Sc}$ & 06833 & 212 & 1085 \\
\hline $\mathrm{N}$ & -0.480375 & -1.62 & -1.58 & $\mathrm{~N}$ & 440 & -1.368988 & 183 & $\mathrm{~N}$ & 533 & 542 & 8286 \\
\hline $\mathrm{H}$ & 0.361027 & -2.03 & -1.98 & $\mathrm{H}$ & 239 & -1.6 & 860 & $\mathrm{H}$ & 851 & 051 & 3667 \\
\hline $\mathrm{H}$ & -1.029358 & -2.419233 & -1.26 & $\mathrm{H}$ & 921 & -2.256400 & 1.636419 & $\mathrm{H}$ & 1396 & 190 & 5548 \\
\hline $\mathrm{H}$ & -0.990535 & -1.258803 & -2.388817 & $\mathrm{H}$ & 0.641675 & -0.909058 & 2.545406 & $\mathrm{H}$ & 6813 & -1.303312 & 2.464585 \\
\hline $\mathrm{N}$ & -0.273255 & 2.439029 & 0.00 & $\mathrm{~N}$ & 145 & 298 & -0.072017 & $\mathrm{~N}$ & 448 & 647 & 0.000787 \\
\hline $\mathrm{H}$ & -0.426884 & 2.818301 & 0.93 & $\mathrm{H}$ & 1.263004 & 040 & -0.8 & $\mathrm{H}$ & & 107 & -0.918128 \\
\hline $\mathrm{H}$ & 0.522040 & 2.95 & -0.36 & $\mathrm{H}$ & & & -0.1 & $\mathrm{H}$ & & 402 & 3964 \\
\hline $\mathrm{H}$ & -1.066938 & 2.764377 & -0.5 & $\mathrm{H}$ & 152 & 2.645339 & 0.7 & $\mathrm{H}$ & & 126 & 0.5 \\
\hline \multicolumn{12}{|c|}{$\mathrm{Sc}\left(\mathrm{NH}_{3}\right)_{6}{ }^{2+}$} \\
\hline $\mathrm{Sc}$ & -0.00 & 000 & 00 & $\mathrm{Sc}$ & 863 & 127 & 622 & $\mathrm{Sc}$ & 231 & 331 & 125 \\
\hline $\mathrm{N}$ & -1.966329 & -0.602201 & -1.292141 & $\mathrm{~N}$ & -0.050551 & -2.410109 & -0.102301 & $\mathrm{~N}$ & 9417 & 4133 & -1.307641 \\
\hline $\mathrm{H}$ & -1.805463 & -1.391083 & -1.91 & $\mathrm{H}$ & -0.29 & -2.79 & -1.0 & $\mathrm{H}$ & & 334 & 636 \\
\hline $\mathrm{H}$ & -2.279362 & 0.14 & -1.9 & $\mathrm{H}$ & & -2.8 & & $\mathrm{H}$ & & & 644 \\
\hline $\mathrm{N}$ & 0.46 & 2.00 & -1.29 & $\mathrm{~N}$ & & -0. & & $\mathrm{~N}$ & & & \\
\hline $\mathrm{H}$ & 0.64 & 2.84 & -0.7 & $\mathrm{H}$ & & -0.0 & & $\mathrm{H}$ & & 29 & 189 \\
\hline $\mathrm{H}$ & -0.30 & 2.25 & -1.9 & $\mathrm{H}$ & & -0.9 & 90 & $\mathrm{H}$ & 74 & 90 & 591 \\
\hline $\mathrm{N}$ & 1.96 & 0.60 & 1.2 & $\mathrm{~N}$ & 35 & 96 & -0.0 & $\mathrm{~N}$ & 33 & 233 & 130 \\
\hline $\mathrm{H}$ & 2.789951 & 0.858335 & 0.7 & $\mathrm{H}$ & -0.4 & 748 & 972 & $\mathrm{H}$ & 559 & 3121 & 684 \\
\hline $\mathrm{H}$ & 2.279361 & -0.143876 & 1.90 & $\mathrm{H}$ & -0.0 & 633 & -0.8 & $\mathrm{H}$ & -0 . & 7183 & 3473 \\
\hline $\mathrm{N}$ & -0.460228 & -2.004908 & 1.25 & $\mathrm{~N}$ & -1 . & 559 & -1.6 & $\mathrm{~N}$ & 583 & 483 & 093 \\
\hline $\mathrm{H}$ & 0.30 & -2.2 & & $\mathrm{H}$ & & & & $\mathrm{H}$ & & & 20 \\
\hline $\mathrm{H}$ & -1.2 & -1.9 & & $\mathrm{H}$ & & -0. & & $\mathrm{H}$ & & & 95 \\
\hline $\mathrm{H}$ & & & -1.5 & $\mathrm{H}$ & & & & $\mathrm{H}$ & & & 316 \\
\hline $\mathrm{H}$ & -2.78 & -0.85 & -0.7 & $\mathrm{H}$ & & -2.8 & & $\mathrm{H}$ & 64 & 20 & 53 \\
\hline $\mathrm{H}$ & -0.647705 & -2.846604 & 26 & $\mathrm{H}$ & -2. & 0.9 & -1.5 & $\mathrm{H}$ & 3273 & 796 & 621 \\
\hline $\mathrm{H}$ & 1.805463 & 1.391084 & 1.9 & $\mathrm{H}$ & 614 & 380 & 988 & $\mathrm{H}$ & $\$ 204$ & -2.194921 & 879 \\
\hline $\mathrm{N}$ & -1.505697 & 1.399325 & $1.2 \mathrm{~S}$ & $\mathrm{~N}$ & 560 & -0.03 & -1.6 & $\mathrm{~N}$ & 940 & 376 & 461 \\
\hline $\mathrm{H}$ & -1.016222 & 2.036580 & 1.9 & $\mathrm{H}$ & 537 & -0.8 & -1 & $\mathrm{H}$ & 838 & 803 & 775 \\
\hline $\mathrm{H}$ & -2.1 & & & $\mathrm{H}$ & & & & $\mathrm{H}$ & & & 16 \\
\hline $\mathrm{H}$ & -2.1 & & & $\mathrm{H}$ & & -0 & & $\mathrm{H}$ & & & 27 \\
\hline $\mathrm{N}$ & & & & $\mathrm{N}$ & & & & $\mathrm{N}$ & & & \\
\hline $\mathrm{H}$ & & -1.9 & & $\mathrm{H}$ & & & & $\mathrm{H}$ & & 31 & 017 \\
\hline $\mathrm{H}$ & 4497 & -0.86 & -1. & $\mathrm{H}$ & -2 & -0.7 & 1.6 & $\mathrm{H}$ & 483 & 543 & 508 \\
\hline $\mathrm{H}$ & 222 & 581 & 010 & $\mathrm{H}$ & 672 & 0.872099 & 1.670561 & $\mathrm{H}$ & -2.552747 & -1.476889 & -0.771166 \\
\hline \multicolumn{12}{|c|}{$\mathrm{Sc}\left(\mathrm{NH}_{3}\right)_{7}^{2+}$} \\
\hline $\mathrm{Sc}$ & 593 & & & $\mathrm{~S}$ & 448 & 0.021978 & & $\mathrm{Sc}$ & 438 & 040 & 067 \\
\hline $\mathrm{N}$ & 0.43 & 0.0 & & $\mathrm{~N}$ & & & & $\mathrm{~N}$ & & 23 & 344 \\
\hline $\mathrm{H}$ & 1.41 & & & $\mathrm{H}$ & & & & $\mathrm{H}$ & & 190 & 396 \\
\hline $\mathrm{H}$ & 0.015168 & -0.715506 & 2.933 & $\mathrm{H}$ & -0.39 & -0.7 & 2.8 & $\mathrm{H}$ & -0.702097 & 0.296068 & 2.96 \\
\hline $\mathrm{N}$ & -2.411067 & -0.167241 & 0.767974 & $\mathrm{~N}$ & -2.412396 & 0.326204 & 0.53 & $\mathrm{~N}$ & 1.285436 & 2.013868 & 0.897245 \\
\hline $\mathrm{H}$ & -2.793961 & 0.703276 & 1.125378 & 1 & -2.805671 & 1.254861 & 0.676485 & $\mathrm{H}$ & 2.197999 & 1.793809 & 1.288692 \\
\hline $\mathrm{H}$ & -2.553315 & -0.832339 & 1.522283 & $\mathrm{H}$ & -2.680069 & -0.196152 & 1.369810 & $\mathrm{H}$ & 0.813075 & 2.508666 & 1.649307 \\
\hline $\mathrm{N}$ & -0.447335 & -0.428760 & -2.390773 & $\mathrm{~N}$ & -0.384992 & -0.164265 & -2.386794 & $\mathrm{~N}$ & -0.025451 & 0.728732 & -2.382847 \\
\hline
\end{tabular}




\begin{tabular}{|c|c|c|c|c|c|c|c|c|c|c|c|}
\hline & & & & & & & & & & & \\
\hline $\mathrm{H}$ & 0.247031 & 0014016 & -2.845877 & $\mathrm{H}$ & -0.022021 & -1.015121 & -2.811010 & $\mathrm{H}$ & -0.919035 & 0.598470 & -2.850013 \\
\hline $\mathrm{H}$ & -0.448870 & 0.429220 & -2.934508 & $\mathrm{H}$ & 0.136402 & 0.589534 & -2.828116 & $\mathrm{H}$ & 0.629122 & 0.212584 & -2.964868 \\
\hline $\mathrm{N}$ & 2.206607 & -1.064923 & -0.513178 & $\mathrm{~N}$ & 1.877296 & -1.522590 & -0.502424 & $\mathrm{~N}$ & -2.137441 & -1.165882 & -0.655796 \\
\hline $\mathrm{H}$ & 2.785148 & -0.443274 & -1.069940 & $\mathrm{H}$ & 2.555803 & -1.025884 & -1.074636 & $\mathrm{H}$ & -1.961611 & -1.952131 & -1.275798 \\
\hline $\mathrm{H}$ & 2.762536 & -1.280156 & 0.310152 & $\mathrm{H}$ & 2.362907 & -1.772409 & 0.356592 & $\mathrm{H}$ & 57629 & -1.567039 & 121005 \\
\hline $\mathrm{H}$ & -3.057948 & -0.453988 & 0.038359 & $\mathrm{H}$ & -2.944862 & -0.081499 & -0.226823 & $\mathrm{H}$ & 81384 & 734443 & 206334 \\
\hline $\mathrm{H}$ & 0.043611 & 428 & & $\mathrm{H}$ & & & & $\mathrm{H}$ & & 412 & \\
\hline $\mathrm{H}$ & 2.181791 & -1.933511 & -1.039114 & $\mathrm{H}$ & 1.714723 & -2.403487 & -0.986074 & $\mathrm{H}$ & -2.829174 & -0.609153 & 50891 \\
\hline $\mathrm{H}$ & -1.339082 & -0.871457 & -2.594215 & $\mathrm{H}$ & -1.343041 & -0.081446 & -2.719174 & $\mathrm{H}$ & 0.204009 & 5306 & 9589 \\
\hline $\mathrm{N}$ & -1.127730 & 2.157124 & -0.627570 & $\mathrm{~N}$ & -0.524410 & 3561 & -0.464483 & $\mathrm{~N}$ & 3644 & 0387 & -0.624545 \\
\hline $\mathrm{H}$ & -1.332563 & 2.780441 & 0.149189 & $\mathrm{H}$ & -0.791309 & 2.851525 & 0.397168 & $\mathrm{H}$ & 3.080225 & -0.566785 & .133454 \\
\hline $\mathrm{H}$ & -2.022829 & 2.001482 & -1.081742 & $\mathrm{H}$ & -1.345777 & 2.415458 & -1.063799 & $\mathrm{H}$ & 6983 & 6547 & 7814 \\
\hline $\mathrm{H}$ & -0.593211 & 2.723834 & 372 & $\mathrm{H}$ & 6955 & 2875 & -0.9 & $\mathrm{H}$ & & 7238 & 9261 \\
\hline $\mathrm{N}$ & -0.3 & -2.4 & & $\mathrm{~N}$ & & & & $\mathrm{~N}$ & & & 902 \\
\hline $\mathrm{H}$ & -1.3 & -2.6 & & $\mathrm{H}$ & 178 & -2. & & $\mathrm{H}$ & 343 & 726 & 515 \\
\hline $\mathrm{H}$ & 067 & -2.8 & 75 & $\mathrm{H}$ & 802 & -2.8 & & $\mathrm{H}$ & 915 & 576 & 979 \\
\hline $\mathrm{H}$ & -0.174736 & -2.958979 & 596 & $\mathrm{H}$ & -0.9 & -2.6 & -0.7 & $\mathrm{H}$ & 013 & 271 & 946 \\
\hline $\mathrm{N}$ & 1.728248 & 1.864479 & 0.139600 & $\mathrm{~N}$ & 7876 & 1.320821 & 0.29 & $\mathrm{~N}$ & 2418 & -2.510620 & 0.105744 \\
\hline $\mathrm{H}$ & 2.011036 & 2.23 & -0.76 & $\mathrm{H}$ & 780 & 811 & -0.6 & $\mathrm{H}$ & & -2.949879 & -0.798078 \\
\hline $\mathrm{H}$ & 293 & 93 & & $\mathrm{H}$ & 967 & & & $\mathrm{H}$ & & & 105 \\
\hline $\mathrm{H}$ & 007 & & 87 & $\mathrm{H}$ & 203 & 839 & & $\mathrm{H}$ & & & \\
\hline \multicolumn{12}{|c|}{$\mathrm{Sc}\left(\mathrm{NH}_{3}\right) 8^{2+}$} \\
\hline $\mathrm{H}$ & 18 & 11 & 32 & $\mathrm{H}$ & 594 & 313 & & $\mathrm{H}$ & -2 & 553 & 296 \\
\hline $\mathrm{H}$ & -1.152539 & -2.688734 & 38 & $\mathrm{H}$ & 047 & 33 & 1.2 & $\mathrm{H}$ & 484 & 52 & 187 \\
\hline $\mathbf{N}$ & -0.350860 & -2.122550 & 164 & $\mathrm{~N}$ & 0.778614 & 070 & 1.4 & $\mathrm{~N}$ & 953 & 073 & 5378 \\
\hline $\mathrm{H}$ & 2.687314 & -1.15 & 402 & $\mathrm{H}$ & 2.915913 & -0.4 & & $\mathrm{H}$ & 3300 & 641 & 2028 \\
\hline $\mathrm{H}$ & -0.45 & -1.9 & 762 & $\mathrm{H}$ & 829 & & & $\mathrm{H}$ & 574 & & \\
\hline $\mathrm{N}$ & & -0.3 & & $\mathrm{~N}$ & & & & $\mathrm{~N}$ & & & \\
\hline $\mathrm{H}$ & 1.9 & -0.4 & & $\mathrm{H}$ & & -0 & & $\mathrm{H}$ & & & 643 \\
\hline $\mathrm{H}$ & 2.76 & 0.4 & 43 & $\mathrm{H}$ & & -1 & & $\mathrm{H}$ & 994 & 240 & 204 \\
\hline $\mathrm{Sc}$ & 0.000000 & 0.000000 & 21 & $\mathrm{Sc}$ & 000 & -0.0 & -0. & $\mathrm{Sc}$ & 0660 & 440 & 0195 \\
\hline $\mathrm{H}$ & -2.764937 & -0.436853 & -1.367194 & $\mathrm{H}$ & -2.039965 & 138 & 25 & $\mathrm{H}$ & 4178 & -2.065293 & 9822 \\
\hline $\mathrm{N}$ & -2.122648 & 215 & 925 & $\mathrm{~N}$ & -2.000833 & 002 & 1.3 & $\mathrm{~N}$ & 9758 & 9601 & 427 \\
\hline $\mathrm{H}$ & -1.900315 & 0.48 & -2.36 & $\mathrm{H}$ & 9782 & 994 & 2.2 & $\mathrm{H}$ & 928 & 491 & 3275 \\
\hline $\mathrm{H}$ & 0.49 & 1.90 & -2.3 & $\mathrm{H}$ & -0.5 & & & $\mathrm{H}$ & & & 30 \\
\hline $\mathrm{N}$ & & & & $\mathrm{N}$ & & & & $\mathrm{N}$ & & 53 & 399 \\
\hline $\mathrm{H}$ & -2.6 & 1.1 & & $\mathrm{H}$ & & & & $\mathrm{H}$ & & 58 & 145 \\
\hline $\mathrm{H}$ & 03 & 2.6 & 55 & $\mathrm{H}$ & -0 . & -2 & & $\mathrm{H}$ & 295 & 75 & 269 \\
\hline $\mathrm{H}$ & -0.436984 & 2.762970 & 63 & $\mathrm{H}$ & 721 & -2.039685 & 25 & $\mathrm{H}$ & 368 & 870 & 7955 \\
\hline $\mathrm{H}$ & 1.090968 & -2.716427 & 136 & $\mathrm{H}$ & 2.422587 & 1155 & -0.93 & $\mathrm{H}$ & -2.541317 & -1.482387 & -1.152979 \\
\hline $\mathrm{H}$ & 2.265151 & -1.641493 & 00 & $\mathrm{H}$ & 472 & 584 & -1 & $\mathrm{H}$ & 7817 & 980 & 3665 \\
\hline $\mathrm{N}$ & 1.255684 & -1.74 & 81 & $\mathrm{~N}$ & 5916 & 16 & -1 . & $\mathrm{N}$ & 0208 & 9644 & 6318 \\
\hline $\mathrm{H}$ & -1.640346 & -2.2 & & $\mathrm{H}$ & -0.1 & & & $\mathrm{H}$ & 483 & 082 & 545 \\
\hline $\mathrm{H}$ & 72 & -1.6 & & $\mathrm{H}$ & 933 & & & $\mathrm{H}$ & 462 & 747 & 2446 \\
\hline $\mathrm{N}$ & -1.7 & 60 & & $\mathrm{~N}$ & & & & $\mathrm{~N}$ & & & 648 \\
\hline $\mathrm{H}$ & -1.6 & -0.9 & & $\mathrm{H}$ & & & & $\mathrm{H}$ & & 36 & 478 \\
\hline $\mathrm{H}$ & -2.716550 & 1.092042 & 27 & $\mathrm{H}$ & -1.7 & 61 & -1.2 & $\mathrm{H}$ & 1.496100 & -2.514884 & -1.178132 \\
\hline $\mathrm{H}$ & 2.716686 & 1.092067 & 74 & $\mathrm{H}$ & 307 & -2.3 & -1.27 & 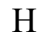 & -1.486003 & 5072 & 2592 \\
\hline $\mathrm{N}$ & 1.748158 & 1.254200 & 1.3 & $\mathrm{~N}$ & 935 & -1.9 & -1 . & $\mathrm{N}$ & 547 & 430 & 2776 \\
\hline $\mathrm{H}$ & 1.692104 & 0.993712 & 2.3 & $\mathrm{H}$ & 736315 & -1.5 & -2.3 & $\mathrm{H}$ & -0.705095 & 2467 & -2.381290 \\
\hline $\mathrm{H}$ & -1.002083 & 1.694595 & 2.363507 & $\mathrm{H}$ & -1.775737 & -1.075631 & -2.303180 & $\mathrm{H}$ & 1.866526 & 0.703606 & -2.382373 \\
\hline $\mathrm{N}$ & -1.255515 & 1.748598 & 1.381982 & $\mathrm{~N}$ & -1.975799 & -0.836133 & -1.334388 & $\mathrm{~N}$ & 2.079165 & 5405 & 92178 \\
\hline $\mathrm{H}$ & 1.640518 & 2.263700 & 1.3 & $\mathrm{H}$ & 0.130787 & -2.710196 & -1.3 & $\mathrm{H}$ & 5985 & 557 & -1.368916 \\
\hline $\mathrm{H}$ & -2.264984 & 1.6 & 1.3 & $\mathrm{H}$ & -2.7 & -0.1 & -1.2 & $\mathrm{H}$ & 86 & -0.063277 & -1.359445 \\
\hline $\mathrm{H}$ & -1.090832 & 2.716460 & 1.12 & $\mathrm{H}$ & -2.42 & -1.66 & -0.9 & $\mathrm{H}$ & 2.526828 & 1.497877 & -1.155471 \\
\hline
\end{tabular}


Table S10: Harmonic vibrational frequencies $\left(\mathrm{cm}^{-1}\right)$ for the $\mathrm{Sc}\left(\mathrm{NH}_{3}\right)_{\mathrm{n}=1-8}{ }^{2+}$ species optimized at CAM-B3LYP, MN15, and MP2 combined with the cc-pVTZ(Sc,N) aug-cc-pVTZ(H) basis sets.

\begin{tabular}{|c|c|c|}
\hline CAM-B3LYP & MN15 & MP2 \\
\hline \multicolumn{3}{|c|}{$\mathrm{Sc}\left(\mathrm{NH}_{3}\right)_{1}{ }^{2+}$} \\
\hline 431 & 424 & 405 \\
\hline 660 & 639 & 643 \\
\hline 661 & 640 & 648 \\
\hline 1413 & 1386 & 1404 \\
\hline 1634 & 1604 & 1644 \\
\hline 1636 & 1605 & 1649 \\
\hline 3374 & 3390 & 3397 \\
\hline 3437 & 3461 & 3483 \\
\hline 3438 & 3461 & 3484 \\
\hline \multicolumn{3}{|c|}{$\mathrm{Sc}\left(\mathrm{NH}_{3}\right)_{2}{ }^{2+}$} \\
\hline $15 i$ & $20 i$ & 13 \\
\hline 59 & 54 & 34 \\
\hline 60 & 55 & 37 \\
\hline 364 & 360 & 336 \\
\hline 421 & 415 & 408 \\
\hline 608 & 590 & 604 \\
\hline 616 & 602 & 607 \\
\hline 637 & 623 & 627 \\
\hline 641 & 628 & 627 \\
\hline 1392 & 1366 & 1385 \\
\hline 1395 & 1369 & 1386 \\
\hline 1647 & 1615 & 1656 \\
\hline 1648 & 1615 & 1657 \\
\hline 1649 & 1618 & 1658 \\
\hline 1651 & 1620 & 1659 \\
\hline 3405 & 3417 & 3418 \\
\hline 3408 & 3421 & 3421 \\
\hline 3471 & 3490 & 3509 \\
\hline 3471 & 3491 & 3509 \\
\hline 3476 & 3496 & 3512 \\
\hline 3477 & 3497 & 3512 \\
\hline \multicolumn{3}{|c|}{$\mathrm{Sc}\left(\mathrm{NH}_{3}\right)_{3}{ }^{2+}$} \\
\hline 1 & 25 & $13 i$ \\
\hline 11 & 32 & 16 \\
\hline 27 & 40 & 26 \\
\hline 62 & 44 & 53 \\
\hline 70 & 56 & 57 \\
\hline 70 & 56 & 58 \\
\hline 328 & 322 & 307 \\
\hline 400 & 394 & 385 \\
\hline 401 & 394 & 386 \\
\hline 569 & 539 & 564 \\
\hline 575 & 541 & 568 \\
\hline 575 & 555 & 568 \\
\hline 611 & 596 & 607 \\
\hline 620 & 603 & 609 \\
\hline 621 & 604 & 610 \\
\hline 1369 & 1339 & 1364 \\
\hline 1369 & 1340 & 1364 \\
\hline 1374 & 1344 & 1368 \\
\hline
\end{tabular}




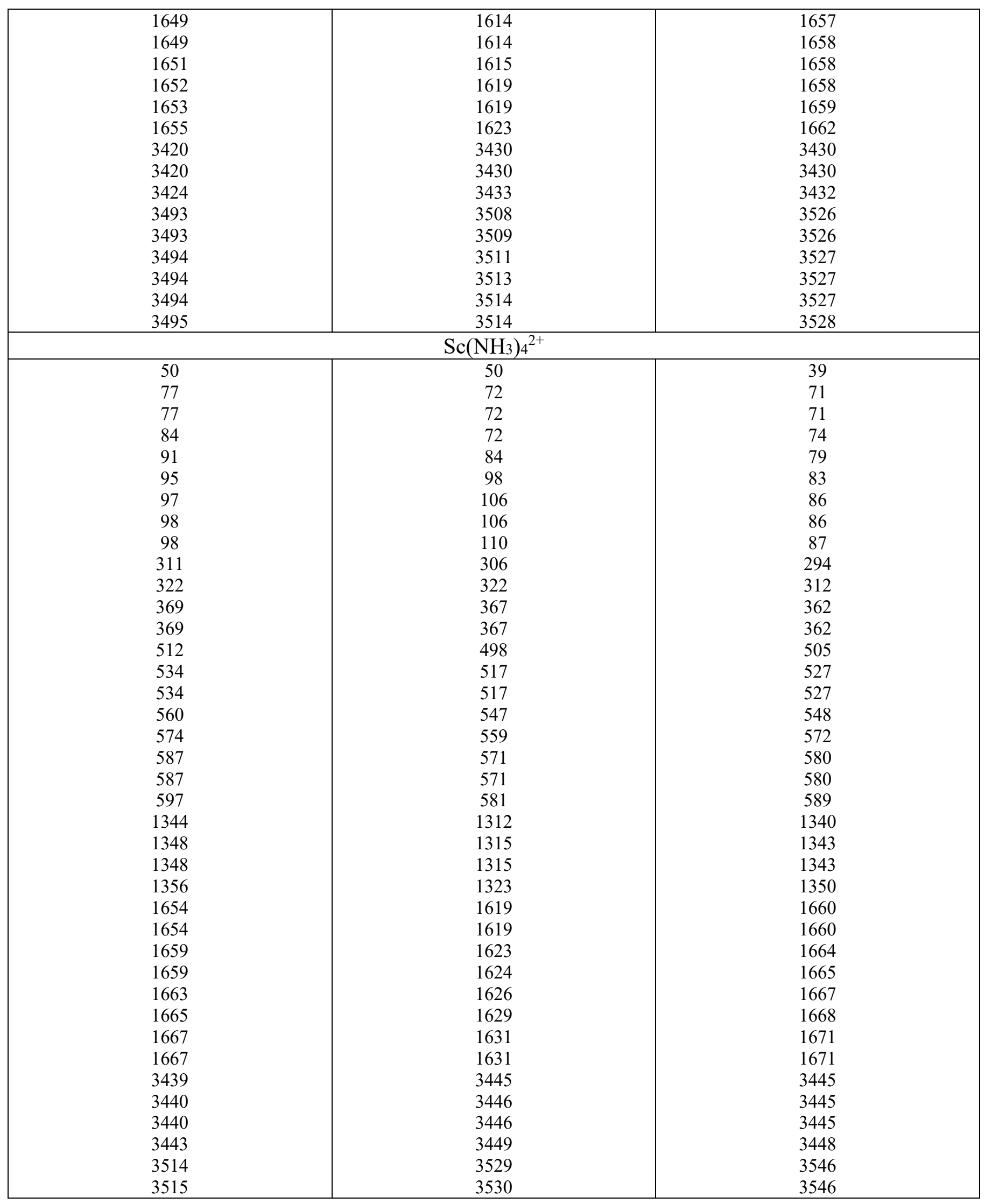




\begin{tabular}{|c|c|c|}
\hline $\begin{array}{l}3515 \\
3516 \\
3519 \\
3520 \\
3520 \\
3520 \\
\end{array}$ & $\begin{array}{l}3530 \\
3531 \\
3534 \\
3535 \\
3535 \\
3535 \\
\end{array}$ & $\begin{array}{l}3546 \\
3548 \\
3548 \\
3548 \\
3548 \\
3548 \\
\end{array}$ \\
\hline \multicolumn{3}{|c|}{$\mathrm{Sc}\left(\mathrm{NH}_{3}\right) 5^{2+}$} \\
\hline 22 & 18 & 23 \\
\hline 35 & 48 & 29 \\
\hline 52 & 70 & 42 \\
\hline 87 & 89 & 76 \\
\hline 95 & 90 & 84 \\
\hline 97 & 102 & 87 \\
\hline 101 & 103 & 90 \\
\hline 106 & 107 & 94 \\
\hline 116 & 114 & 104 \\
\hline 122 & 137 & 108 \\
\hline 130 & 142 & 117 \\
\hline 146 & 146 & 130 \\
\hline 253 & 261 & 250 \\
\hline 290 & 286 & 277 \\
\hline 296 & 307 & 296 \\
\hline 324 & 326 & 322 \\
\hline 339 & 343 & 335 \\
\hline 444 & 409 & 439 \\
\hline 475 & 433 & 468 \\
\hline 488 & 455 & 480 \\
\hline 498 & 479 & 493 \\
\hline 515 & 490 & 503 \\
\hline 549 & 524 & 534 \\
\hline 559 & 533 & 554 \\
\hline 575 & 555 & 564 \\
\hline 576 & 566 & 566 \\
\hline 582 & 567 & 568 \\
\hline 1316 & 1277 & 1313 \\
\hline 1321 & 1281 & 1318 \\
\hline 1326 & 1283 & 1322 \\
\hline 1326 & 1287 & 1323 \\
\hline 1338 & 1295 & 1333 \\
\hline 1657 & 1618 & 1662 \\
\hline 1658 & 1622 & 1662 \\
\hline 1659 & 1622 & 1663 \\
\hline 1664 & 1626 & 1667 \\
\hline 1664 & 1627 & 1667 \\
\hline 1670 & 1634 & 1672 \\
\hline 1674 & 1636 & 1675 \\
\hline 1675 & 1638 & 1677 \\
\hline 1678 & 1641 & 1680 \\
\hline 1680 & 1643 & 1681 \\
\hline 3454 & 3462 & 3456 \\
\hline 3454 & 3462 & 3456 \\
\hline 3456 & 3463 & 3457 \\
\hline 3457 & 3464 & 3458 \\
\hline 3459 & 3466 & 3459 \\
\hline 3532 & 3548 & 3559 \\
\hline
\end{tabular}




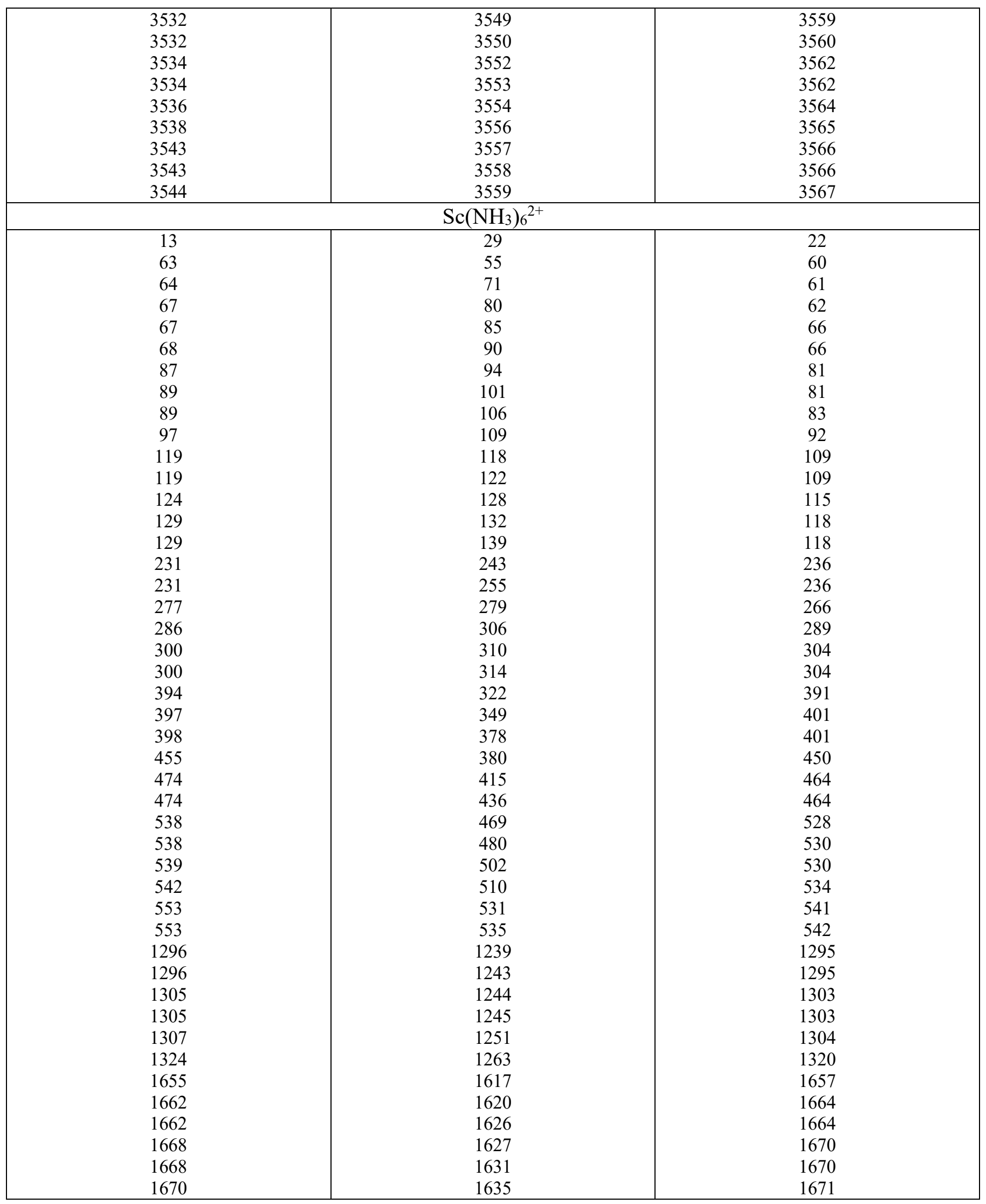




\begin{tabular}{|c|c|c|}
\hline & & \\
\hline 1671 & 1637 & 1673 \\
\hline 1672 & 1638 & 1673 \\
\hline 1673 & 1638 & 1674 \\
\hline 1680 & 1641 & 1681 \\
\hline 1680 & 1648 & 1681 \\
\hline 1686 & 1657 & 1687 \\
\hline 3465 & 3472 & 3464 \\
\hline 3465 & 3474 & 3464 \\
\hline 3467 & 3477 & 3465 \\
\hline 3467 & 3478 & 3465 \\
\hline 3467 & 3478 & 3465 \\
\hline 3470 & 3480 & 3466 \\
\hline 3545 & 3564 & 3572 \\
\hline 3546 & 3565 & 3572 \\
\hline 3546 & 3567 & 3572 \\
\hline 3547 & 3567 & 3573 \\
\hline 3547 & 3568 & 3573 \\
\hline 3549 & 3569 & 3573 \\
\hline 3551 & 3571 & 3573 \\
\hline 3551 & 3572 & 3574 \\
\hline 3553 & 3572 & 3575 \\
\hline 3553 & 3573 & 3575 \\
\hline 3553 & 3573 & 3575 \\
\hline 3553 & 3574 & 3575 \\
\hline \multicolumn{3}{|c|}{$\mathrm{Sc}\left(\mathrm{NH}_{3}\right) 7^{2+}$} \\
\hline 15 & 20 & N/A \\
\hline 32 & 51 & \\
\hline 48 & 86 & \\
\hline 58 & 101 & \\
\hline 75 & 111 & \\
\hline 83 & 129 & \\
\hline 98 & 132 & \\
\hline 105 & 137 & \\
\hline 113 & 142 & \\
\hline 123 & 152 & \\
\hline 134 & 157 & \\
\hline 136 & 165 & \\
\hline 144 & 174 & \\
\hline 150 & 181 & \\
\hline 158 & 186 & \\
\hline 163 & 190 & \\
\hline 165 & 203 & \\
\hline 171 & 218 & \\
\hline 203 & 226 & \\
\hline 206 & 233 & \\
\hline 216 & 237 & \\
\hline 238 & 260 & \\
\hline 249 & 267 & \\
\hline 259 & 276 & \\
\hline 275 & 289 & \\
\hline 331 & 298 & \\
\hline 348 & 309 & \\
\hline 361 & 325 & \\
\hline 377 & 351 & \\
\hline 393 & 372 & \\
\hline
\end{tabular}




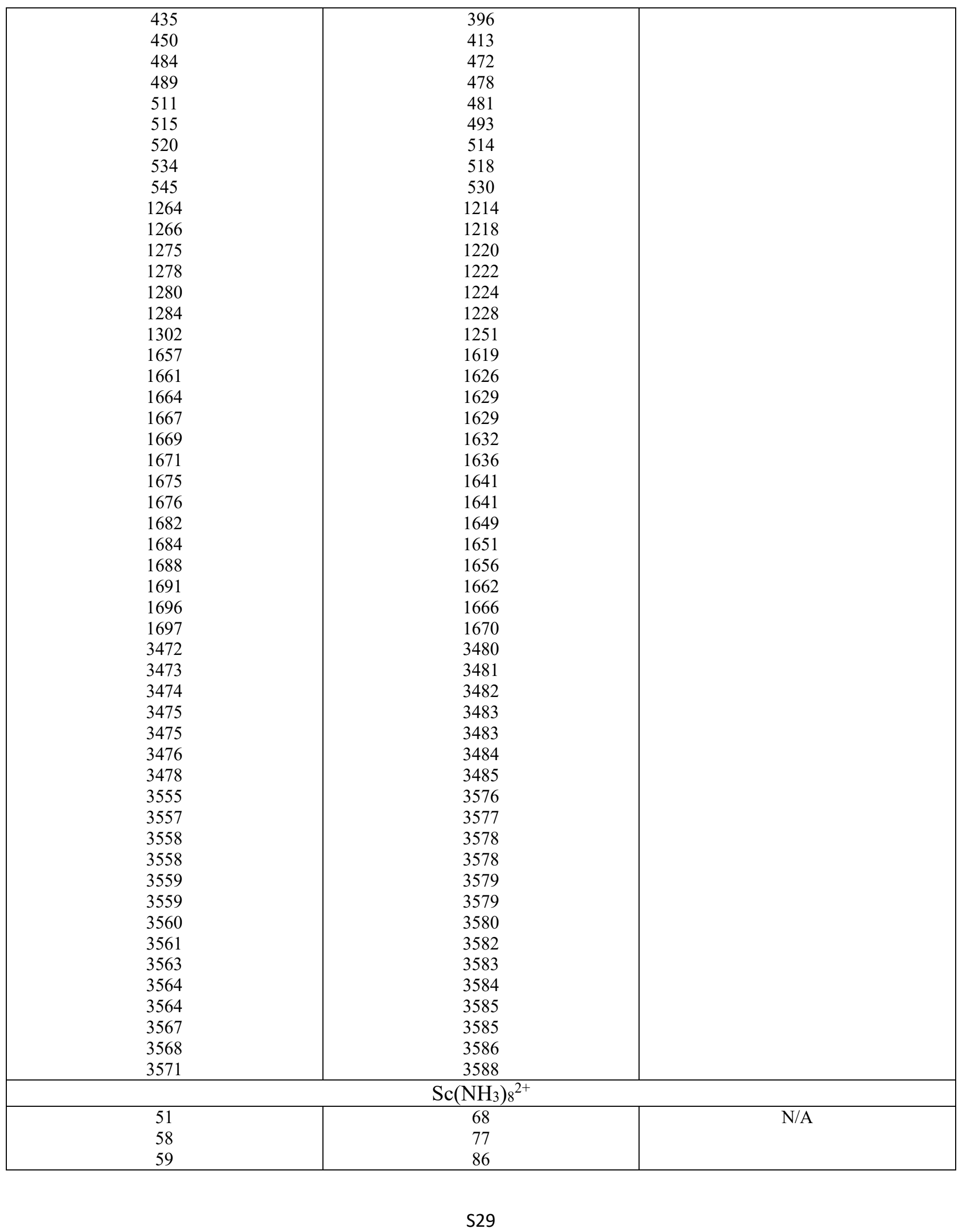




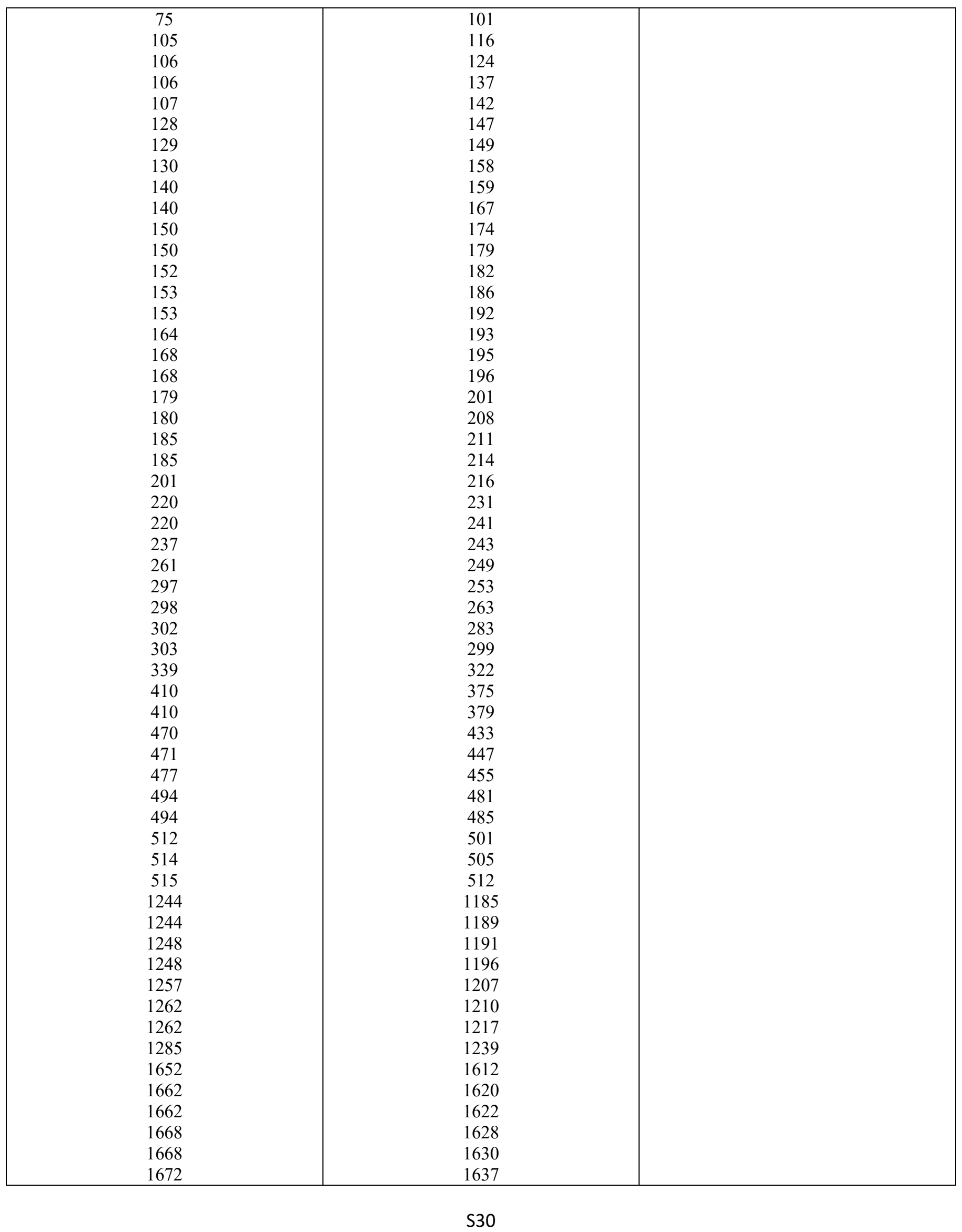




\begin{tabular}{|l|l|l|}
\hline 1679 & 1639 \\
1679 & 1640 \\
1681 & 1642 \\
1681 & 1644 \\
1689 & 1651 \\
1692 & 1660 \\
1692 & 1661 \\
1700 & 1662 \\
1700 & 1664 \\
1701 & 1667 \\
3479 & 3478 \\
3479 & 3479 \\
3480 & 3480 \\
3480 & 3481 \\
3480 & 3482 \\
3480 & 3482 \\
3481 & 3484 \\
3481 & 3485 \\
3567 & 3576 \\
3568 & 3576 \\
3568 & 3577 \\
3569 & 3577 \\
3569 & 3582 \\
3569 & 3583 \\
3569 & 3584 \\
3569 & 3585 \\
3572 & 3585 \\
3572 & 3585 \\
3572 & 3587 & \\
3572 & 3587 \\
3572 & 3589 \\
3572 & 3594 & \\
& 3596 & \\
\hline
\end{tabular}


Table S11: Cartesian coordinates $(\AA)$ for the $\mathrm{Sc}\left(\mathrm{NH}_{3}\right)_{\mathrm{n}=1-6}{ }^{2+}$ species optimized at C-MP2 combined with the cc-pwCVTZ(Sc)/cc-pVTZ(N)/aug-cc-pVTZ(H) basis sets.

\begin{tabular}{|c|c|c|c|c|c|c|c|c|c|c|c|}
\hline \multicolumn{4}{|c|}{$\mathrm{Sc}\left(\mathrm{NH}_{3}\right)_{1^{2+}}$} & \multicolumn{4}{|c|}{$\mathrm{Sc}\left(\mathrm{NH}_{3}\right)_{2}{ }^{2+}$} & \multicolumn{4}{|c|}{$\mathrm{Sc}\left(\mathrm{NH}_{3}\right)_{3}{ }^{2+}$} \\
\hline $\mathrm{N}$ & -1.478323 & -0.000070 & 0.000000 & $\mathrm{~N}$ & -2.181588 & -0.343897 & 0.000016 & $\mathrm{H}$ & 0.015189 & 2.698788 & -0.939365 \\
\hline $\mathrm{H}$ & -1.902873 & 0.908129 & -0.214551 & $\mathrm{H}$ & -2.733588 & -0.037894 & 0.805240 & $\mathrm{~N}$ & 0.013732 & 2.285704 & -0.004670 \\
\hline $\mathrm{H}$ & -1.904694 & -0.639361 & -0.678750 & $\mathrm{H}$ & -2.733662 & -0.037854 & -0.805141 & $\mathrm{H}$ & -0.787246 & 2.716550 & 0.460697 \\
\hline $\mathrm{H}$ & -1.904718 & -0.267735 & 0.893146 & $\mathrm{H}$ & -2.290613 & -1.360771 & -0.000004 & $\mathrm{H}$ & 0.820888 & 2.706857 & 0.458892 \\
\hline \multirow[t]{10}{*}{$\mathrm{Sc}$} & 0.764788 & -0.000026 & 0.000007 & $\mathrm{~N}$ & 2.181588 & -0.343896 & 0.000035 & $\mathrm{~N}$ & 1.981622 & -1.151295 & -0.004582 \\
\hline & & & & $\mathrm{H}$ & 2.733541 & -0.038234 & 0.805420 & $\mathrm{H}$ & 2.728435 & -0.700535 & -0.536406 \\
\hline & & & & $\mathrm{H}$ & 2.290596 & -1.360772 & -0.000398 & $\mathrm{H}$ & 2.383870 & -1.297098 & 0.923464 \\
\hline & & & & $\mathrm{H}$ & 2.733722 & -0.037530 & -0.804961 & $\mathrm{H}$ & 1.928241 & -2.089305 & -0.405778 \\
\hline & & & & $\mathrm{Sc}$ & 0.000000 & 0.366076 & -0.000024 & $\mathrm{~N}$ & -1.995236 & -1.127708 & -0.004615 \\
\hline & & & & & & & & $\mathrm{H}$ & -2.739873 & -0.662784 & -0.527213 \\
\hline & & & & & & & & $\mathrm{H}$ & -1.955157 & -2.062008 & -0.415894 \\
\hline & & & & & & & & $\mathrm{H}$ & -2.393746 & -1.278425 & 0.924259 \\
\hline & & & & & & & & $\mathrm{Sc}$ & -0.000068 & -0.003759 & 0.007353 \\
\hline & \multicolumn{3}{|c|}{$\mathrm{Sc}\left(\mathrm{NH}_{3}\right)_{4}{ }^{2+}$} & \multicolumn{4}{|c|}{$\mathrm{Sc}\left(\mathrm{NH}_{3}\right) 5^{2+}$} & & & & \\
\hline $\mathrm{Sc}$ & -0.000004 & 0.000004 & 0.000001 & $\mathrm{~N}$ & -0.953980 & -1.492631 & -1.487188 & & & & \\
\hline $\mathrm{N}$ & 1.572895 & 1.555478 & 0.718129 & $\mathrm{H}$ & -0.266675 & -2.107965 & -1.918962 & & & & \\
\hline $\mathrm{H}$ & 1.180754 & 2.295872 & 1.299592 & $\mathrm{H}$ & -1.653578 & -2.126893 & -1.105546 & & & & \\
\hline $\mathrm{H}$ & 2.074279 & 2.051555 & -0.019217 & $\mathrm{H}$ & -1.417655 & -1.055567 & -2.282411 & & & & \\
\hline $\mathrm{N}$ & 1.555508 & -1.572805 & -0.718244 & $\mathrm{~N}$ & -2.249351 & 0.736380 & 0.400811 & & & & \\
\hline $\mathrm{H}$ & 1.155130 & -2.308919 & -1.299527 & $\mathrm{H}$ & -2.726469 & 1.204267 & -0.368777 & & & & \\
\hline $\mathrm{H}$ & 2.051555 & -2.074272 & 0.019068 & $\mathrm{H}$ & -2.859646 & -0.046649 & 0.628150 & & & & \\
\hline $\mathrm{N}$ & -1.572829 & -1.555527 & 0.718166 & $\mathrm{H}$ & -2.361854 & 1.367989 & 1.191364 & & & & \\
\hline $\mathrm{H}$ & -2.074142 & -2.051695 & -0.019169 & $\mathrm{~N}$ & 2.339358 & -0.145109 & -0.454522 & & & & \\
\hline $\mathrm{H}$ & -2.309066 & -1.155130 & 1.299280 & $\mathrm{H}$ & 2.683512 & 0.470063 & -1.189180 & & & & \\
\hline $\mathrm{N}$ & -1.555567 & 1.572848 & -0.718052 & $\mathrm{H}$ & 2.969424 & 0.019788 & 0.329302 & & & & \\
\hline $\mathrm{H}$ & -2.295906 & 1.180687 & -1.299571 & $\mathrm{H}$ & 2.591837 & -1.076210 & -0.781235 & & & & \\
\hline $\mathrm{H}$ & -2.051711 & 2.074133 & 0.019316 & $\mathrm{Sc}$ & 0.009487 & 0.087248 & -0.004874 & & & & \\
\hline $\mathrm{H}$ & 2.295925 & -1.180589 & -1.299626 & $\mathrm{~N}$ & 0.360999 & -1.662448 & 1.551879 & & & & \\
\hline $\mathrm{H}$ & 2.309072 & 1.155066 & 1.299308 & $\mathrm{H}$ & -0.500564 & -1.992625 & 1.983428 & & & & \\
\hline $\mathrm{H}$ & -1.155200 & 2.309107 & -1.299160 & $\mathrm{H}$ & 0.812353 & -2.509505 & 1.211205 & & & & \\
\hline $\mathrm{H}$ & -1.180659 & -2.295849 & 1.299701 & $\mathrm{H}$ & 0.931356 & -1.374720 & 2.345761 & & & & \\
\hline & & & & $\mathrm{N}$ & 0.485074 & 2.411239 & -0.000194 & & & & \\
\hline & & & & $\mathrm{H}$ & 0.707287 & 2.779661 & -0.924356 & & & & \\
\hline & & & & $\mathrm{H}$ & -0.267499 & 3.009641 & 0.333928 & & & & \\
\hline & & & & $\mathrm{H}$ & 1.284232 & 2.674508 & 0.574188 & & & & \\
\hline
\end{tabular}


Table S12: Electronic energies (a.u.) for the $\mathrm{Sc}\left(\mathrm{NH}_{3}\right)_{\mathrm{n}=1-8}{ }^{+}$species (singlet spin multiplicity) optimized at CAM-B3LYP, MN15, and MP2 combined with the cc-pVTZ(Sc,N)/aug-cc-pVTZ(H) basis sets.

\begin{tabular}{crcc}
\hline$n$ & CAM-B3LYP & MN15 & MP2 \\
\hline 0 & -760.333362 & -760.277306 & -759.486196 \\
1 & -817.026807 & -816.921873 & -816.041488 \\
2 & -873.654519 & -873.505695 & -872.570545 \\
3 & -930.252121 & -930.060283 & -929.067816 \\
4 & -986.845973 & -986.611444 & -985.566189 \\
5 & -1043.419653 & -1043.145786 & -1042.048839 \\
6 & -1099.990031 & -1099.680897 & -1098.531307 \\
$6^{a}$ & -1099.989906 & & \\
7 & -1156.555525 & -1156.216789 & -1154.999705 \\
8 & -1213.118738 & -1212.747535 & -1211.471829 \\
\hline
\end{tabular}

${ }^{a}$ Energies of the imposed $\mathrm{C}_{2 \mathrm{~h}}$ structure.

Table S13: Electronic energies (a.u.) for the $\mathrm{Sc}\left(\mathrm{NH}_{3}\right)_{\mathrm{n}=1-8^{+}}$species (triplet spin multiplicity) optimized at CAM-B3LYP, MN15, and MP2 combined with the cc-pVTZ(Sc,N)/aug-cc-pVTZ(H) basis sets.

\begin{tabular}{crcc}
\hline$n$ & CAM-B3LYP & MN15 & MP2 \\
\hline 0 & -760.416756 & -760.348246 & -759.545873 \\
1 & -817.049015 & -816.935590 & -816.065009 \\
2 & -873.666113 & -873.495171 & -872.562466 \\
3 & -930.258092 & -930.059141 & -929.068470 \\
4 & -986.843878 & -986.607287 & -985.556467 \\
5 & -1043.421124 & -1043.144954 & -1042.041305 \\
6 & -1100.003118 & -1099.690776 & -1098.527440 \\
$6^{a}$ & -1100.003128 & & \\
7 & -1156.569014 & -1156.225576 & -1154.990197 \\
8 & -1213.134757 & -1212.756478 & -1211.465506 \\
$8^{a}$ & -1213.134774 & & \\
\hline
\end{tabular}

${ }^{a}$ Energies of the imposed $\mathrm{C}_{2 \mathrm{~h}}$ or $\mathrm{C}_{2 \mathrm{v}}$ structures for $n=6$ or 8 . 
Table S14: Cartesian coordinates $(\AA)$ for the $\mathrm{Sc}\left(\mathrm{NH}_{3}\right)_{\mathrm{n}=1-8}{ }^{+}$species (singlet spin multiplicity) optimized at CAM-B3LYP, MN15, and MP2 with the cc-pVTZ(Sc,N) aug-cc-pVTZ(H) basis sets.

\begin{tabular}{|c|c|c|c|c|c|c|c|c|c|c|c|}
\hline \multicolumn{4}{|c|}{ CAM-B3LYP } & \multicolumn{4}{|c|}{ MN15 } & \multicolumn{4}{|c|}{ MP2 } \\
\hline \multicolumn{12}{|c|}{$\mathrm{Sc}\left(\mathrm{NH}_{3}\right)_{1}{ }^{+}$} \\
\hline $\mathrm{Sc}$ & -0.746274 & 0.000001 & -0.000001 & $\mathrm{Sc}$ & 0.744515 & -0.000002 & -0.000001 & Sc & -0.752536 & 0.000000 & -0.000001 \\
\hline $\mathrm{N}$ & 1.451310 & -0.000025 & -0.000004 & $\mathrm{~N}$ & -1.448129 & 0.000037 & -0.000006 & $\mathrm{~N}$ & 1.462241 & 0.000000 & -0.000004 \\
\hline $\mathrm{H}$ & 1.837499 & 0.463527 & 0.822690 & $\mathrm{H}$ & -1.832599 & -0.454918 & 0.831780 & $\mathrm{H}$ & 1.855816 & 0.094336 & 0.937067 \\
\hline $\mathrm{H}$ & 1.837562 & 0.480863 & -0.812653 & $\mathrm{H}$ & -1.832676 & -0.493125 & -0.809685 & $\mathrm{H}$ & 1.855888 & 0.764341 & -0.550209 \\
\hline $\mathrm{H}$ & 1.837529 & -0.944244 & -0.009994 & $\mathrm{H}$ & -1.832635 & 0.947823 & -0.022034 & $\mathrm{H}$ & 1.855863 & -0.858684 & -0.386810 \\
\hline \multicolumn{12}{|c|}{$\mathrm{Sc}\left(\mathrm{NH}_{3}\right)_{2}{ }^{+}$} \\
\hline $\mathrm{Sc}$ & 0.000000 & -0.000332 & -0.000547 & Sc & -0.000130 & 0.000046 & -0.000001 & Sc & -0.000000 & 0.000082 & 0.000030 \\
\hline $\mathrm{N}$ & 2.254422 & 0.000376 & 0.000522 & $\mathrm{~N}$ & 0.000191 & -0.012564 & 186 & $\mathrm{~N}$ & 2.263616 & -0.000088 & -0.000013 \\
\hline $\mathrm{H}$ & 2.640200 & -0.745644 & -0.576386 & $\mathrm{H}$ & -0.577228 & 0.736459 & 2.632313 & $\mathrm{H}$ & 2.656959 & 0.828532 & -0.445268 \\
\hline $\mathrm{H}$ & 2.639882 & -0.126488 & 0.935124 & $\mathrm{H}$ & 0.939416 & 0.109942 & 2.628780 & $\mathrm{H}$ & 2.657062 & -0.799921 & -0.495030 \\
\hline $\mathrm{H}$ & 2.640531 & 0.872951 & -0.356801 & $\mathrm{H}$ & -0.361482 & -0.890247 & 2.62 & $\mathrm{H}$ & 2.657245 & -0.028799 & 0095 \\
\hline $\mathrm{H}$ & -2.640982 & 0.621732 & -0.70 & $\mathrm{H}$ & 117 & -0.607799 & -2.6 & $\mathrm{H}$ & 379 & 0.396258 & 2900 \\
\hline $\mathrm{N}$ & -2.254422 & 0.000360 & 0.0 & $\mathrm{~N}$ & 0.000031 & 0.012430 & -2.2 & $\mathrm{~N}$ & -2.2 & -0.000071 & 002 \\
\hline $\mathrm{H}$ & -2.639292 & 0.303981 & & $\mathrm{H}$ & 0.896316 & -0.292769 & -2.6 & $\mathrm{H}$ & -2.6 & -0.937074 & 327 \\
\hline $\mathrm{H}$ & -2.640346 & -0.924708 & -0.1 & $\mathrm{H}$ & -0.1 & 0.944383 & & $\mathrm{H}$ & -2.6 & 0.540396 & \\
\hline \multicolumn{12}{|c|}{$\mathrm{Sc}\left(\mathrm{NH}_{3}\right)_{3}{ }^{+}$} \\
\hline $\mathrm{N}$ & -1.474317 & -1.519006 & -0.0 & $\mathrm{~N}$ & 337 & -1.466453 & -0.0 & $\mathrm{~N}$ & & -1.478749 & 598 \\
\hline $\mathrm{H}$ & -1.164526 & -2.486322 & -0.0 & $\mathrm{H}$ & 76 & -2.446261 & -0.0 & $\mathrm{H}$ & & -2.455758 & 895 \\
\hline $\mathrm{H}$ & -2.067192 & -1.417929 & 61 & $\mathrm{H}$ & 22 & -1.338080 & -0.8 & $\mathrm{H}$ & 88 & -1.372209 & 587 \\
\hline $\mathrm{H}$ & -2.067384 & -1.417400 & -0.82 & $\mathrm{H}$ & 2.08 & -1.338376 & 06 & $\mathrm{H}$ & 170 & -1.372373 & 774 \\
\hline $\mathrm{N}$ & -1.473682 & 1.519391 & -0.00 & $\mathrm{~N}$ & $1.4 \mathrm{C}$ & 1.467824 & -0.0 & $\mathrm{~N}$ & & 1.477820 & 0571 \\
\hline $\mathrm{H}$ & -2.066652 & 1.418034 & -0.825806 & $\mathrm{H}$ & 2.087521 & 1.339737 & & $\mathrm{H}$ & 2.109688 & 1.370152 & 0.819910 \\
\hline $\mathrm{H}$ & -2.066726 & 1.418506 & 0.822169 & $\mathrm{H}$ & 2.081612 & 1.341020 & -0.83 & $\mathrm{H}$ & 2.109335 & 1.371113 & -0.821413 \\
\hline $\mathrm{H}$ & -1.163525 & 2.486588 & -0.001997 & $\mathrm{H}$ & 1.21 & 2.447238 & -0.00 & $\mathrm{H}$ & 295 & 2.455165 & 0.000161 \\
\hline $\mathrm{N}$ & 509 & -0.000128 & -0.0 & $\mathrm{~N}$ & -2.5 & 0.000103 & -0.0 & $\mathrm{~N}$ & & 0.00 & 586 \\
\hline $\mathrm{H}$ & 2.888708 & -0.813787 & -0 & $\mathrm{H}$ & -2.8 & 0.871993 & -0. & $\mathrm{H}$ & & 0.86 & 772 \\
\hline $\mathrm{H}$ & 2.889073 & 0.817359 & -0.4 & $\mathrm{H}$ & -2.88 & -0.755175 & -0.5 & $\mathrm{H}$ & 630 & -0.745766 & 401 \\
\hline $\mathrm{H}$ & 2.846573 & -0.004168 & 38 & $\mathrm{H}$ & -2.859043 & -0.125387 & & $\mathrm{H}$ & -2.887177 & -0.128012 & 0.943223 \\
\hline $\mathrm{Sc}$ & 0.244385 & -0.000128 & 438 & $\mathrm{Sc}$ & -0.264629 & -0.000335 & & $\mathrm{Sc}$ & & 0.000577 & 0.000395 \\
\hline \multicolumn{12}{|c|}{$\mathrm{Sc}\left(\mathrm{NH}_{3}\right)_{4}{ }^{+}$} \\
\hline $\mathrm{Sc}$ & 000 & -0.000000 & 63 & Sc & -0.000000 & 0.000002 & & $\mathrm{Sc}$ & -0 . & -0.000005 & 180 \\
\hline $\mathrm{N}$ & -1.728641 & -1.541250 & -0 . & $\mathrm{N}$ & -1.7 & -1.501469 & & $\mathrm{~N}$ & & -1.530931 & 245 \\
\hline $\mathrm{H}$ & -2.313086 & -1.413513 & -0.8 & $\mathrm{H}$ & -2.3 & -1.346854 & -0.8 & $\mathrm{H}$ & 93 & -1.409927 & 964 \\
\hline $\mathrm{H}$ & -2.312657 & -1.413919 & 186 & $\mathrm{H}$ & -2.32 & -1.347033 & & $\mathrm{H}$ & 5003 & -1.409912 & 4806 \\
\hline $\mathrm{N}$ & 1.728643 & -1.541249 & 0.000022 & $\mathrm{~N}$ & 1.749860 & -1.501468 & -0.000229 & $\mathrm{~N}$ & 1.734692 & -1.530930 & -0.000111 \\
\hline $\mathrm{H}$ & 2.313108 & -1.413538 & -0.825886 & $\mathrm{H}$ & 2.326142 & -1.346793 & -0.832048 & $\mathrm{H}$ & 2.325411 & -1.409848 & -0.824367 \\
\hline $\mathrm{H}$ & 1.453067 & -2.518195 & -0.000285 & $\mathrm{H}$ & 1.506213 & -2.489440 & -0.000415 & $\mathrm{H}$ & 1.469161 & -2.512122 & -0.000258 \\
\hline $\mathrm{N}$ & 1.728640 & 1.541250 & -0.000249 & $\mathrm{~N}$ & 1.749864 & 1.501467 & -0.000111 & $\mathrm{~N}$ & 1.734679 & 1.530936 & 0.000097 \\
\hline $\mathrm{H}$ & 1.453068 & 2.518196 & -0.000383 & $\mathrm{H}$ & 6220 & 2.489440 & -0.0 & $\mathrm{H}$ & 142 & 2.512126 & 0.000117 \\
\hline $\mathrm{H}$ & 2.312857 & 1.413604 & -0.8 & $\mathrm{H}$ & & 1.346879 & & $\mathrm{H}$ & & 1.409980 & -0.8 \\
\hline $\mathrm{N}$ & -1.728643 & 1.541248 & & $\mathrm{~N}$ & -1.74 & 1.501466 & -0.0 & $\mathrm{~N}$ & -1.73 & 1.530935 & 074 \\
\hline $\mathrm{H}$ & -1.453063 & 2.518193 & -0.00 & $\mathrm{H}$ & -1. & 2.489439 & -0.00 & $\mathrm{H}$ & 142 & 2.512125 & -0.000035 \\
\hline $\mathrm{H}$ & -2.313099 & 1.413601 & -0.825740 & $\mathrm{H}$ & -2.326197 & 1.346816 & -0.83 & $\mathrm{H}$ & -2.325435 & 1.409897 & -0.824164 \\
\hline $\mathrm{H}$ & 2.312639 & -1.413892 & 0.826323 & $\mathrm{H}$ & 2.326099 & -1.347094 & 0.831678 & $\mathrm{H}$ & 2.325087 & -1.409992 & 0.824402 \\
\hline $\mathrm{H}$ & -1.453067 & -2.518196 & -0.000428 & $\mathrm{H}$ & -1.506213 & -2.489441 & -0.000103 & $\mathrm{H}$ & -1.469161 & -2.512123 & 0.000165 \\
\hline $\mathrm{H}$ & -2.312649 & 1.413831 & 0.826468 & $\mathrm{H}$ & -2.326052 & 1.347064 & 0.831903 & $\mathrm{H}$ & -2.325041 & 1.409961 & 0.824606 \\
\hline $\mathrm{H}$ & 2.312884 & 1.413824 & 0.825862 & $\mathrm{H}$ & 2.326155 & 1.347000 & 0.831743 & $\mathrm{H}$ & 2.325130 & 1.409877 & 0.824552 \\
\hline \multicolumn{12}{|c|}{$\mathrm{Sc}\left(\mathrm{NH}_{3}\right) 5^{+}$} \\
\hline $\mathrm{N}$ & -0.929336 & -2.000869 & -0.761256 & $\mathrm{~N}$ & -0.736640 & -2.146256 & -0.589164 & $\mathrm{~N}$ & -0.908070 & -1.988216 & -0.762289 \\
\hline
\end{tabular}




\begin{tabular}{|c|c|c|c|c|c|c|c|c|c|c|c|}
\hline $\mathrm{H}$ & 1.828296 & -1.823871 & -1.201703 & $\mathrm{H}$ & -1.688080 & -2.130918 & -0.955460 & $\mathrm{H}$ & -1.818144 & -1.838351 & -1.194730 \\
\hline $\mathrm{H}$ & -0.345716 & -2.396977 & -1.492053 & $\mathrm{H}$ & -0.153313 & -2.525295 & -1.333987 & $\mathrm{H}$ & -0.333550 & -2.385290 & -1.502770 \\
\hline $\mathrm{H}$ & -1.080422 & -2.729985 & -0.061252 & $\mathrm{H}$ & -0.716622 & -2.803869 & 0.197692 & $\mathrm{H}$ & -1.041490 & -2.725069 & -0.063218 \\
\hline $\mathrm{N}$ & -2.079381 & 0.021820 & 1.040443 & $\mathrm{~N}$ & -2.083783 & -0.076202 & 0.995555 & $\mathrm{~N}$ & -2.049591 & 0.000571 & 1.061386 \\
\hline $\mathrm{H}$ & -2.919932 & 0.035221 & 0.458846 & $\mathrm{H}$ & -2.920995 & -0.110108 & 0.404497 & $\mathrm{H}$ & -2.892364 & 0.001176 & 8342 \\
\hline $\mathrm{H}$ & -2.154479 & -0.786158 & 1.651947 & $\mathrm{H}$ & -2.123000 & -0.869237 & 1.634932 & $\mathrm{H}$ & -2.130309 & -0.803509 & 1.680722 \\
\hline $\mathrm{H}$ & -2.133898 & 0.825166 & 1.660094 & $\mathrm{H}$ & -2.156308 & 0.749986 & 1.589553 & $\mathrm{H}$ & -2.129668 & 0.804094 & 1.681519 \\
\hline $\mathrm{N}$ & 1.889810 & 1.452295 & 0.209813 & $\mathrm{~N}$ & 1.659017 & 1.632340 & 0.346935 & $\mathrm{~N}$ & 1.861279 & 1.470140 & 0.215905 \\
\hline $\mathrm{H}$ & 1.649508 & 2.410806 & 0.445251 & $\mathrm{H}$ & 1.239487 & 2.479678 & 0.729071 & $\mathrm{H}$ & 1.627201 & 2.445029 & 0.389887 \\
\hline $\mathrm{H}$ & 2.570446 & 1.162822 & 0.906889 & $\mathrm{H}$ & 2.418089 & 1.386083 & 0.980539 & $\mathrm{H}$ & 2.499046 & 1.214081 & 0.968294 \\
\hline $\mathrm{H}$ & 2.364057 & 1.477497 & -0.696598 & $\mathrm{H}$ & 2.070882 & 1.868715 & -0.565046 & $\mathrm{H}$ & 2.398096 & 1.455798 & -0.657327 \\
\hline $\mathrm{Sc}$ & 0.072293 & -0.001433 & 0.016484 & $\mathrm{Sc}$ & 0.064697 & -0.031048 & 0.004081 & $\mathrm{Sc}$ & 0.071471 & 0.000213 & 0.021146 \\
\hline $\mathrm{N}$ & 1.84 & -1.5( & 0.23 & $\mathrm{~N}$ & 301 & -1.245452 & 0.096067 & $\mathrm{~N}$ & 1.857853 & -1.473727 & 0.218198 \\
\hline $\mathrm{H}$ & 1.594699 & -2.477990 & 0.379792 & $\mathrm{H}$ & 2.036041 & -2.253994 & -0.040062 & $\mathrm{H}$ & 1389 & -2.448017 & 0.392319 \\
\hline $\mathrm{H}$ & 2.411733 & -1.474259 & -0.620841 & $\mathrm{H}$ & 2.713182 & -0.86 & 785 & $\mathrm{H}$ & 131 & -1.461033 & 4153 \\
\hline $\mathrm{H}$ & 2.447110 & -1.254469 & 1.013551 & $\mathrm{H}$ & 2.398637 & -1.080420 & 1.043737 & $\mathrm{H}$ & 2.494942 & -1.218633 & 0.971492 \\
\hline $\mathrm{N}$ & -0.874427 & 2.032453 & -0.737826 & $\mathrm{~N}$ & -1.011967 & 1.879996 & -0.860196 & $\mathrm{~N}$ & -0.905479 & 1.990834 & -0.759502 \\
\hline $\mathrm{H}$ & -0.285336 & 2.430190 & -1.463366 & $\mathrm{H}$ & -0.411938 & 2.349458 & -1.537081 & $\mathrm{H}$ & -0.331222 & 2.387704 & -1.500301 \\
\hline $\mathrm{H}$ & -1.777711 & 1.880413 & -1.178809 & $\mathrm{H}$ & -1.821819 & 1.560339 & -1.392849 & $\mathrm{H}$ & -1.8 & 1.843316 & -1.190775 \\
\hline $\mathrm{H}$ & -1.010008 & 2.752715 & -0.025249 & $\mathrm{H}$ & -1.339389 & 2.583201 & -0.188831 & $\mathrm{H}$ & 399 & 7021 & 258 \\
\hline \multicolumn{12}{|c|}{$\mathrm{Sc}\left(\mathrm{NH}_{3}\right)_{6}{ }^{+}$} \\
\hline Sc & -0.085828 & 0.013379 & -0.003827 & Sc & -0.157843 & 0.000018 & 0433 & Sc & 7241 & 2963 & 0202 \\
\hline $\mathrm{N}$ & 1.847722 & -0.704749 & 1.245588 & $\mathrm{~N}$ & 1.675960 & 0.588671 & -1.418401 & $\mathrm{~N}$ & 1.807206 & 0.876254 & -1.191265 \\
\hline $\mathrm{H}$ & 1.593712 & -1.475193 & 1.857148 & $\mathrm{H}$ & 1.513709 & 1.535295 & -1.762325 & $\mathrm{H}$ & 1.506050 & 1.610260 & -1.830076 \\
\hline $\mathrm{H}$ & 2.157878 & 0.031726 & 1.872560 & $\mathrm{H}$ & 1.611908 & -0.000167 & -2.246127 & $\mathrm{H}$ & 2.241560 & 0.181495 & -1.795737 \\
\hline $\mathrm{N}$ & 1.734386 & 1.097976 & -1.145900 & $\mathrm{~N}$ & 1.677146 & -0.580051 & 457 & $\mathrm{~N}$ & 7852 & 2938 & 8189 \\
\hline $\mathrm{H}$ & 2.26 & 0.4 & 51 & $\mathrm{H}$ & 619 & & 74 & $\mathrm{H}$ & 2.2 & 723 & 415 \\
\hline $\mathrm{H}$ & 2.414571 & 658 & $-0.5^{\prime}$ & $\mathrm{H}$ & 853 & 891 & 706 & $\mathrm{H}$ & 935 & 3800 & 3396 \\
\hline $\mathrm{N}$ & -2.097652 & 0.91 & -0.82 & $\mathrm{~N}$ & -1.9 & 5016 & 095 & $\mathrm{~N}$ & 069 & 0518 & 617 \\
\hline $\mathrm{H}$ & -2.765740 & 1.197145 & -0.093088 & $\mathrm{H}$ & -2.554075 & -1.562229 & -0.102100 & $\mathrm{H}$ & -2.574708 & -1.539112 & 0.139459 \\
\hline $\mathrm{H}$ & -2.568776 & 0.249450 & -1.422740 & $\mathrm{H}$ & -2.523570 & -0.658071 & 1.302639 & $\mathrm{H}$ & -2.477242 & -0.586811 & 1.471709 \\
\hline $\mathrm{N}$ & -1.713282 & -1.474283 & 0.848998 & $\mathrm{~N}$ & -1.973426 & 1.243244 & -0.687736 & $\mathrm{~N}$ & -1.906066 & 1.218909 & -0.848973 \\
\hline $\mathrm{H}$ & -2.311372 & -1.914634 & 0.14 & $\mathrm{H}$ & -2.566041 & 1.545341 & 190 & $\mathrm{H}$ & 664 & 1579 & -0.135842 \\
\hline $\mathrm{H}$ & -1.31 & -2.2 & 1.4 & $\mathrm{H}$ & -1.7 & & & $\mathrm{H}$ & 65 & 63 & 967 \\
\hline $\mathrm{H}$ & 1.3 & 1.7 & -1.7 & $\mathrm{H}$ & 1.5 & -1. & & $\mathrm{H}$ & & -1.6 & 887 \\
\hline $\mathrm{H}$ & $2.6^{\circ}$ & -1.0 & 0.7 & $\mathrm{H}$ & 2.6 & 98 & 60 & $\mathrm{H}$ & 2.5 & 1.2 & 821 \\
\hline $\mathrm{H}$ & -2.323154 & -0.969074 & 1.48 & $\mathrm{H}$ & -2.525882 & 0.640516 & 3974 & $\mathrm{H}$ & -2.469514 & 0.635614 & -1.467033 \\
\hline $\mathrm{H}$ & -1.933541 & 1.735803 & -1.403941 & $\mathrm{H}$ & -1.724042 & -2.076682 & 1.241601 & $\mathrm{H}$ & -1.662809 & -1.979256 & 1.426809 \\
\hline $\mathrm{N}$ & 0.485504 & -2.075872 & -1.028807 & $\mathrm{~N}$ & 0.446831 & 2.161292 & 0.780437 & $\mathrm{~N}$ & 0.224609 & 2.153267 & 0.968195 \\
\hline $\mathrm{H}$ & 1.348569 & -2.034096 & -1.563355 & $\mathrm{H}$ & 1.429197 & 2.257798 & 1.037668 & $\mathrm{H}$ & 1.085851 & 2.249809 & 1.503633 \\
\hline $\mathrm{H}$ & -0.236215 & -2.297716 & -1.709140 & $\mathrm{H}$ & -0.100494 & 2.273395 & 1.636017 & $\mathrm{H}$ & -0.518200 & 2.327392 & 1.643779 \\
\hline $\mathrm{H}$ & 0. & -2.8 & -0.4 & $\mathrm{H}$ & & & & $\mathrm{H}$ & 58 & 617 & 26 \\
\hline $\mathrm{N}$ & -0.08 & 2.2 & & $\mathrm{~N}$ & 77 & -2.1 & & $\mathrm{~N}$ & & -2.1 & 004 \\
\hline $\mathrm{H}$ & -0.1 & 2.9 & & $\mathrm{H}$ & & -2.9 & & $\mathrm{H}$ & & & -0. \\
\hline $\mathrm{H}$ & 3701 & 2.42 & 1.4 & $\mathrm{H}$ & 1.444931 & -2.248233 & 19 & $\mathrm{H}$ & 1.0 & -2.2 & 978 \\
\hline $\mathrm{H}$ & -0.856229 & 2.282257 & 1.567299 & $\mathrm{H}$ & -0.082342 & -2.265939 & -1.640587 & $\mathrm{H}$ & -0.570271 & -2.302024 & 537 \\
\hline \multicolumn{12}{|c|}{$\mathrm{Sc}\left(\mathrm{NH}_{3}\right)_{7}^{+}$} \\
\hline $\mathrm{Sc}$ & 0.000114 & 0.080932 & 455 & $\mathrm{Sc}$ & -0.001418 & 0.062264 & -0.024791 & & & $\mathrm{~N} / \mathrm{A}$ & \\
\hline $\mathrm{N}$ & 1.813908 & -0.477947 & -1.499905 & $\mathrm{~N}$ & 1.630604 & -0.377050 & -1.659783 & & & & \\
\hline $\mathrm{H}$ & 1.708341 & -1.32 & -2.0 & $\mathrm{H}$ & 905 & 643 & -2.2 & & & & \\
\hline $\mathrm{H}$ & 2.748174 & -0.530736 & -1.070280 & $\mathrm{H}$ & 2.621521 & -0.324517 & -1.369874 & & & & \\
\hline $\mathrm{N}$ & 1.457845 & 1.985495 & -0.043956 & $\mathrm{~N}$ & 1.426236 & 1.946732 & 0.015604 & & & & \\
\hline $\mathrm{H}$ & 1.304864 & 2.523325 & -0.896578 & $\mathrm{H}$ & 1.219313 & 2.677344 & -0.667331 & & & & \\
\hline $\mathrm{H}$ & 2.456212 & 1.768558 & -0.016200 & $\mathrm{H}$ & 2.438019 & 1.774466 & -0.021610 & & & & \\
\hline $\mathrm{N}$ & -1.732702 & -0.412992 & 1.579232 & $\mathrm{~N}$ & -1.520125 & -0.260408 & & & & & \\
\hline
\end{tabular}




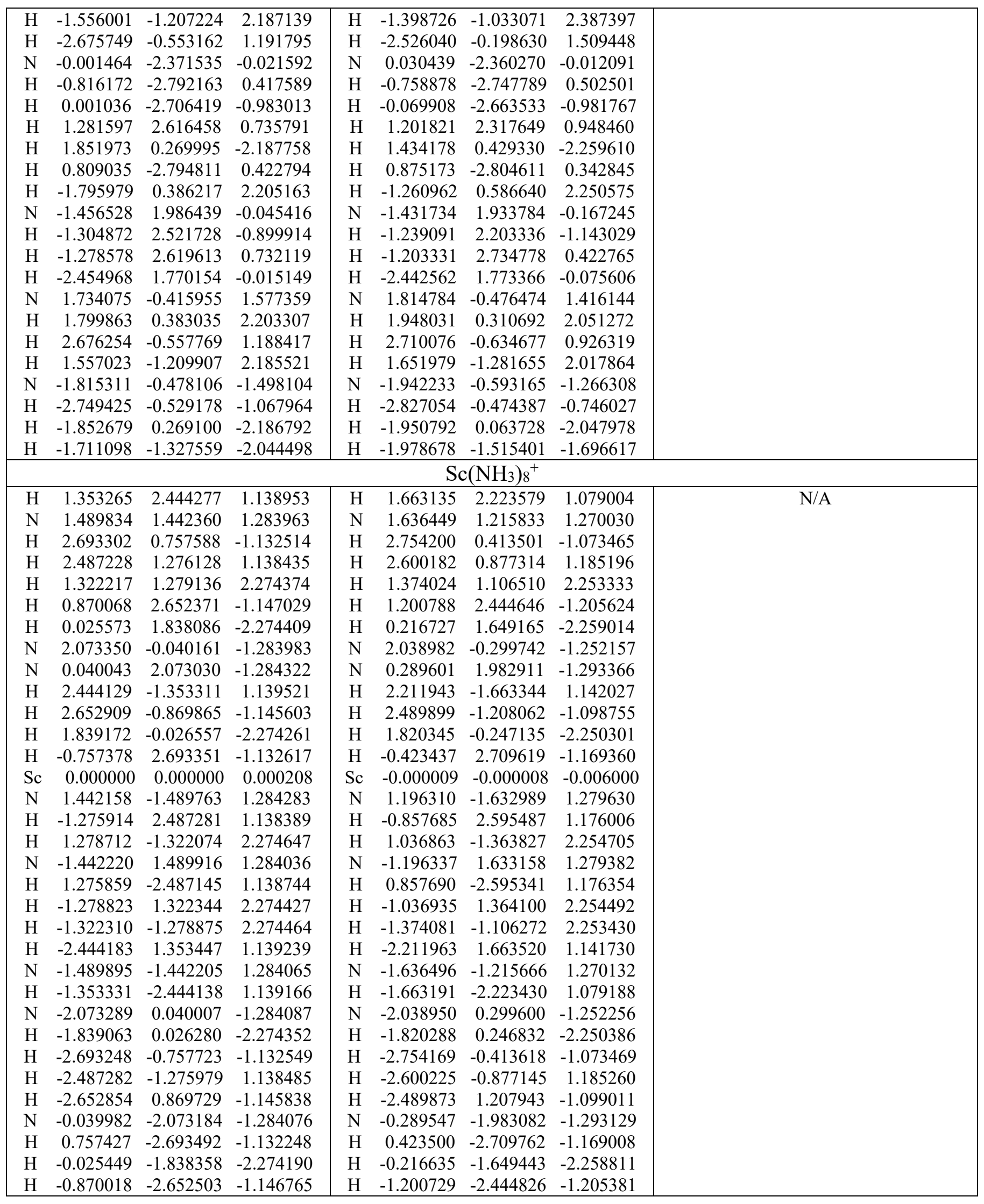


Table S15: Cartesian coordinates $(\AA)$ for the $\mathrm{Sc}\left(\mathrm{NH}_{3}\right)_{\mathrm{n}=1-8}{ }^{+}$species (triplet spin multiplicity) optimized at CAM-B3LYP, MN15, and MP2 with the cc-pVTZ(Sc,N) aug-cc-pVTZ(H) basis sets.

\begin{tabular}{|c|c|c|c|c|c|c|c|c|c|c|c|}
\hline \multicolumn{4}{|c|}{ CAM-B3LYP } & \multicolumn{4}{|c|}{ MN15 } & \multicolumn{4}{|c|}{ MP2 } \\
\hline \multicolumn{12}{|c|}{$\mathrm{Sc}\left(\mathrm{NH}_{3}\right)_{1}{ }^{+}$} \\
\hline $\mathrm{Sc}$ & 0.771684 & 0.000004 & 0.000001 & $\mathrm{Sc}$ & 0.773306 & -0.000016 & 0.000024 & $\mathrm{Sc}$ & -0.797235 & -0.000000 & 0.000016 \\
\hline $\mathrm{N}$ & -1.502188 & -0.000072 & -0.000027 & $\mathrm{~N}$ & -1.505946 & -0.000022 & 0.000013 & $\mathrm{~N}$ & 1.552666 & 0.000001 & 0.000027 \\
\hline $\mathrm{H}$ & -1.896670 & 0.386937 & 0.856474 & $\mathrm{H}$ & -1.898254 & 0.846551 & -0.415671 & $\mathrm{H}$ & 1.957356 & -0.772869 & -0.526309 \\
\hline $\mathrm{H}$ & -1.896651 & -0.935135 & -0.093261 & $\mathrm{H}$ & -1.900278 & -0.063148 & 0.940387 & $\mathrm{H}$ & 1.958489 & -0.069280 & 0.932106 \\
\hline $\mathrm{H}$ & -1.896726 & 0.548612 & -0.763040 & $\mathrm{H}$ & -1.899281 & -0.782922 & -0.525302 & $\mathrm{H}$ & 1.957432 & 0.842148 & -0.406333 \\
\hline \multicolumn{12}{|c|}{$\mathrm{Sc}\left(\mathrm{NH}_{3}\right)_{2}{ }^{+}$} \\
\hline $\mathrm{Sc}$ & -0.000000 & 0.000039 & -0.000013 & $\mathrm{H}$ & -2.650182 & 0.703632 & 0.808945 & Sc & -0.000001 & 0.000005 & 0.000049 \\
\hline $\mathrm{N}$ & -2.326702 & -0.000125 & -0.000018 & $\mathrm{~N}$ & -2.306780 & 0.187334 & -0.000201 & $\mathrm{~N}$ & -2.393391 & 0.000672 & -0.002768 \\
\hline $\mathrm{H}$ & -2.715993 & 0.935362 & 0.095727 & $\mathrm{H}$ & -2.793780 & -0.710034 & 0.016700 & $\mathrm{H}$ & -2.776018 & -0.077956 & 0.937988 \\
\hline $\mathrm{H}$ & -2.718763 & -0.549711 & 0.761780 & $\mathrm{H}$ & -2.655750 & 0.676406 & -0.823740 & $\mathrm{H}$ & -2.806976 & -0.771522 & -0.520714 \\
\hline $\mathrm{H}$ & -2.719556 & -0.384190 & -0.856935 & $\mathrm{Sc}$ & 0.000015 & -0.188685 & -0.0 & $\mathrm{H}$ & -2.813347 & 0.842636 & -0.389977 \\
\hline $\mathrm{H}$ & 2.718844 & 0.550324 & -0.76 & $\mathrm{H}$ & 2.792780 & -0.710654 & & $\mathrm{H}$ & 978 & 0.771029 & 321 \\
\hline $\mathrm{N}$ & 2.326700 & 0.000054 & 0.00 & $\mathrm{~N}$ & 2.306825 & 0.187409 & -0.0 & $\mathrm{~N}$ & 393 & -0.000693 & 671 \\
\hline $\mathrm{H}$ & 2.715913 & -0.935377 & -0.0 & $\mathrm{H}$ & 2.65 & 0.693880 & & $\mathrm{H}$ & & 0.078847 & -0.9 \\
\hline $\mathrm{H}$ & 2.719569 & 0.383265 & 0.85 & $\mathrm{H}$ & 1095 & 0.685958 & -0.8 & $\mathrm{H}$ & 406 & -0.843002 & \\
\hline \multicolumn{12}{|c|}{$\mathrm{Sc}\left(\mathrm{NH}_{3}\right)_{3}{ }^{+}$} \\
\hline $\mathrm{N}$ & 1.544421 & -1.575318 & -0.0 & $\mathrm{~N}$ & 1.52 & -1.592497 & -0.0 & $\mathrm{~N}$ & 00 & 1.554076 & 167 \\
\hline $\mathrm{H}$ & 1.164430 & -2.517443 & -0.0 & $\mathrm{H}$ & 1.13 & -2.532407 & -0.0 & $\mathrm{H}$ & 97 & 1.490832 & 586 \\
\hline $\mathrm{H}$ & 2.149740 & -1.523129 & -0.8 & $\mathrm{H}$ & 2.1 & -1.541976 & -0.8 & $\mathrm{H}$ & 04 & 1.490636 & -0 \\
\hline $\mathrm{H}$ & 2.149710 & -1.523145 & 0.8 & $\mathrm{H}$ & 2.13 & -1.541984 & & $\mathrm{H}$ & & 2.511993 & -0 . \\
\hline $\mathrm{N}$ & 1.497218 & 1.604814 & 0.000 & $\mathrm{~N}$ & 1.46 & 1.632895 & 0.00 & $\mathrm{~N}$ & -2.62 & 0.006217 & -0.000321 \\
\hline $\mathrm{H}$ & 2.103646 & 1.567134 & 0.816117 & $\mathrm{H}$ & 2.068864 & 1.601021 & 0.82 & $\mathrm{H}$ & -3.014912 & 0.487940 & 0.807721 \\
\hline $\mathrm{H}$ & 2.103660 & 1.567154 & -0.816061 & $\mathrm{H}$ & 2.068881 & 1.601034 & -0.82 & $\mathrm{H}$ & -3.015301 & 0.467515 & -0.819998 \\
\hline $\mathrm{H}$ & 1.094260 & 2.537329 & 0.000031 & $\mathrm{H}$ & 1.040890 & 2.559536 & & $\mathrm{H}$ & 717 & -0.927887 & 0.011639 \\
\hline $\mathrm{N}$ & -2.569498 & -0.009345 & 0.0 & $\mathrm{~N}$ & -2.5 & -0.014665 & & $\mathrm{~N}$ & & -1.583683 & -0. \\
\hline $\mathrm{H}$ & -2.948834 & -0.483397 & 0.8 & $\mathrm{H}$ & -2.9 & -0.492579 & & $\mathrm{H}$ & & -1.533239 & 856 \\
\hline $\mathrm{H}$ & -2.967218 & 0.926853 & -0.00 & $\mathrm{H}$ & -2.958159 & 0.921806 & -0.0 & $\mathrm{H}$ & 1.1 & -2.533641 & 518 \\
\hline $\mathrm{H}$ & -2.948861 & -0.483695 & -0.81 & $\mathrm{H}$ & -2.928595 & -0.492801 & -0.8 & $\mathrm{H}$ & 2.156828 & -1.534813 & -0.812924 \\
\hline $\mathrm{Sc}$ & -0.247882 & -0.009939 & -0.000010 & $\mathrm{Sc}$ & -0.229759 & -0.012466 & & $\mathrm{Sc}$ & -0. & 0.011638 & 0.000304 \\
\hline \multicolumn{12}{|c|}{$\mathrm{Sc}\left(\mathrm{NH}_{3}\right)_{4}^{+}$} \\
\hline Sc & 000 & -0.000000 & & $\mathrm{Sc}$ & 0.000075 & 0.000053 & & Sc & & -0.00 & 039 \\
\hline $\mathrm{N}$ & -1.815967 & -1.563066 & 0.0 & $\mathrm{H}$ & -1.4 & -2.542212 & & $\mathrm{H}$ & & -2.52 & 397 \\
\hline $\mathrm{H}$ & -2.416265 & -1.481887 & -0.8 & $\mathrm{~N}$ & -1.7 & -1.593332 & -0.0 & $\mathrm{~N}$ & 298 & -1.563632 & 068 \\
\hline $\mathrm{H}$ & -2.415928 & -1.482068 & 0.81 & $\mathrm{H}$ & -2.372197 & -1.521231 & 55 & $\mathrm{H}$ & -2.448844 & -1.481639 & 0.815182 \\
\hline $\mathrm{N}$ & 1.815967 & -1.563065 & -0.000141 & $\mathrm{H}$ & -2.369013 & -1.522684 & -0.824002 & $\mathrm{H}$ & -2.448547 & -1.482073 & -0.815311 \\
\hline $\mathrm{H}$ & 2.416199 & -1.481847 & -0.817739 & $\mathrm{~N}$ & 1.771351 & 1.593522 & -0.000745 & $\mathrm{~N}$ & 1.843196 & 1.563615 & 0.000073 \\
\hline $\mathrm{H}$ & 1.478166 & -2.520224 & -0.000340 & $\mathrm{H}$ & 2.372469 & 1.521249 & 0.819910 & $\mathrm{H}$ & 2.448937 & 1.481458 & 0.815031 \\
\hline $\mathrm{N}$ & 1.815966 & 1.563066 & -0.000075 & $\mathrm{H}$ & 1.403209 & 2.542342 & 0.001193 & $\mathrm{H}$ & 1.528538 & 2.530006 & 0.000619 \\
\hline $\mathrm{H}$ & 1.478167 & 2.520225 & -0.000127 & $\mathrm{~N}$ & 0761 & -1.593956 & & $\mathrm{~N}$ & 574 & -1.563336 & -0.000091 \\
\hline $\mathrm{H}$ & 2.416101 & 1.481937 & -0.81 & $\mathrm{H}$ & 1.402457 & -2.542715 & -0.0 & $\mathrm{H}$ & 03 & -2.529754 & 0.000189 \\
\hline $\mathrm{N}$ & -1.815966 & 1.563066 & & $\mathrm{H}$ & 2.36 & -1.523243 & & $\mathrm{H}$ & 290 & -1.481316 & 0.814894 \\
\hline $\mathrm{H}$ & -1.478161 & 2.520223 & -0.00 & $\mathrm{~N}$ & -1.770984 & 1.593669 & 0.0 & $\mathrm{~N}$ & 3479 & 1.563449 & -0.000095 \\
\hline $\mathrm{H}$ & -2.416206 & 1.481899 & -0.817555 & $\mathrm{H}$ & -2.371234 & 1.522109 & -0.820674 & $\mathrm{H}$ & -2.448518 & 1.481819 & -0.815622 \\
\hline $\mathrm{H}$ & 2.415994 & -1.482109 & 0.817634 & $\mathrm{H}$ & -2.369445 & 1.522517 & 0.823395 & $\mathrm{H}$ & -2.449223 & 1.481418 & 0.814865 \\
\hline $\mathrm{H}$ & -1.478166 & -2.520225 & -0.000090 & $\mathrm{H}$ & 2.371327 & -1.522217 & -0.820424 & $\mathrm{H}$ & 2.448627 & -1.481553 & -0.815596 \\
\hline $\mathrm{H}$ & -2.415987 & 1.482063 & 0.817817 & $\mathrm{H}$ & 2.368918 & 1.523122 & -0.824164 & $\mathrm{H}$ & 2.448284 & 1.482143 & -0.815442 \\
\hline $\mathrm{H}$ & 2.416090 & 1.482017 & 0.817619 & $\mathrm{H}$ & -1.402916 & 2.542524 & 0.000033 & $\mathrm{H}$ & -1.528776 & 2.529821 & 0.000291 \\
\hline \multicolumn{12}{|c|}{$\mathrm{Sc}\left(\mathrm{NH}_{3}\right) 5^{+}$} \\
\hline $\mathrm{N}$ & 0.003688 & -0.058957 & 2.199384 & $\mathrm{~N}$ & 0.000973 & -0.108865 & 2.115782 & $\mathrm{~N}$ & 0.000991 & -0.059038 & 2.166228 \\
\hline
\end{tabular}




\begin{tabular}{|c|c|c|c|c|c|c|c|c|c|c|c|}
\hline & & & & & & & & & & & \\
\hline $\mathrm{H}$ & -0.804392 & 0.414545 & 2.593245 & $\mathrm{H}$ & -0.811844 & 0.368496 & 2.499230 & $\mathrm{H}$ & -0.804096 & 0.409971 & 2.571569 \\
\hline $\mathrm{H}$ & 0.005413 & -0.991621 & 2.601990 & $\mathrm{H}$ & 0.001245 & -1.051007 & 2.499949 & $\mathrm{H}$ & 0.002027 & -0.989438 & 2.573928 \\
\hline $\mathrm{H}$ & 0.812169 & 0.416304 & 2.590302 & $\mathrm{H}$ & 0.813832 & 0.368795 & 2.498767 & $\mathrm{H}$ & 0.805769 & 0.411354 & 2.570578 \\
\hline $\mathrm{N}$ & 1.814342 & -1.551108 & -0.431177 & $\mathrm{~N}$ & 1.779514 & -1.552892 & -0.411876 & $\mathrm{~N}$ & 1.841310 & -1.583673 & -0.398883 \\
\hline $\mathrm{H}$ & 2.609506 & -1.480753 & 0.201246 & $\mathrm{H}$ & 2.551868 & -1.495096 & 0.253113 & $\mathrm{H}$ & 2.636599 & -1.531937 & 0.234448 \\
\hline $\mathrm{H}$ & 1.497556 & -2.515065 & -0.385709 & $\mathrm{H}$ & 1.425628 & -2.507461 & -0.374930 & $\mathrm{H}$ & 1.533555 & -2.552310 & -0.376031 \\
\hline $\mathrm{H}$ & 2.173945 & -1.430583 & -1.376865 & $\mathrm{H}$ & & -1.436141 & -1.349804 & $\mathrm{H}$ & +204 & -1.457441 & 9017 \\
\hline $\mathrm{N}$ & -1.818141 & 1.569948 & -0.382256 & $\mathrm{~N}$ & -1.780769 & 1.589676 & -0.328391 & $\mathrm{~N}$ & -1.845708 & 1.601853 & -0.353108 \\
\hline $\mathrm{H}$ & -1.497162 & 2.530028 & -0.297940 & $\mathrm{H}$ & -1.419057 & 2.534137 & -0.203412 & $\mathrm{H}$ & -1.534938 & 2.567422 & -0.283026 \\
\hline $\mathrm{H}$ & -2.167384 & 1.485989 & -1.335810 & $\mathrm{H}$ & -2.131837 & 1.549498 & -1.288009 & $\mathrm{H}$ & -2.200550 & 1.517098 & -1.304907 \\
\hline $\mathrm{H}$ & -2.621655 & 1.481058 & 0.237634 & $\mathrm{H}$ & -2.582705 & 1.493216 & 0.296864 & $\mathrm{H}$ & -2.654241 & 1.527272 & 0.261226 \\
\hline $\mathrm{Sc}$ & -0.000571 & 0.007940 & -0.281012 & $\mathrm{Sc}$ & -0.000165 & 0.013538 & -0.315456 & $\mathrm{Sc}$ & -0.000181 & 0.008892 & -0.321736 \\
\hline $\mathrm{N}$ & -1.816085 & -1.550693 & -0.427479 & $\mathrm{~N}$ & -1.780309 & -1.552489 & 0622 & $\mathrm{~N}$ & -1.842098 & -1.583302 & 7291 \\
\hline $\mathrm{H}$ & -1.499227 & -2.5 & -0.3 & $\mathrm{H}$ & -1. & 086 & & $\mathrm{H}$ & 608 & & 0660 \\
\hline $\mathrm{H}$ & -2.60 & -1.480050 & 786 & $\mathrm{H}$ & -2.5 & -1.494117 & 88 & $\mathrm{H}$ & 472 & 518 & 4409 \\
\hline $\mathrm{H}$ & -2.177933 & -1.4 & -1.3 & $\mathrm{H}$ & -2.1 & -1.4 & -1 . & $\mathrm{H}$ & 3137 & 550 & 8490 \\
\hline $\mathrm{N}$ & 1.817275 & 1.569707 & -0.383823 & $\mathrm{~N}$ & 1.780918 & 1.589301 & -0.32 & $\mathrm{~N}$ & 1.845824 & 486 & -0.352914 \\
\hline $\mathrm{H}$ & 1.496624 & 2.529776 & -0.298154 & $\mathrm{H}$ & 1.419555 & 2.533755 & -0.202468 & $\mathrm{H}$ & 1.536688 & 2.567233 & -0.278250 \\
\hline $\mathrm{H}$ & 2.621781 & 1.480121 & 0.234676 & $\mathrm{H}$ & 2.583300 & 1.492124 & 0.2 & $\mathrm{H}$ & 2.656186 & 3492 & 0.258571 \\
\hline $\mathrm{H}$ & 2.164956 & 1.486470 & -1.33 & $\mathrm{H}$ & 2.13 & 1.549779 & 408 & $\mathrm{H}$ & 7570 & 574 & 5115 \\
\hline \multicolumn{12}{|c|}{$\mathrm{Sc}\left(\mathrm{NH}_{3}\right)_{6}{ }^{+}$} \\
\hline $\mathrm{Sc}$ & 0.0 & -0.0 & & $\mathrm{Sc}$ & -0.0 & 042 & -0. & $\mathrm{Sc}$ & 315 & 009 & 549 \\
\hline $\mathrm{N}$ & -1.680885 & 1.108894 & 022 & $\mathrm{~N}$ & 700 & 866 & -0.2 & $\mathrm{~N}$ & 1238 & 588 & 3327 \\
\hline $\mathrm{H}$ & -1.273099 & 1.799537 & 1.938821 & $\mathrm{H}$ & -0.828967 & 2.840184 & 0.150759 & $\mathrm{H}$ & 0.826359 & -2.853897 & 0.393175 \\
\hline $\mathrm{H}$ & -2.193454 & 0.461427 & 1.907400 & $\mathrm{H}$ & 0.027675 & 2.550889 & -1.217727 & $\mathrm{H}$ & -0.028486 & -2.853293 & -0.983122 \\
\hline $\mathrm{N}$ & -0.166386 & -2.045560 & 1.249592 & $\mathrm{~N}$ & -1.889850 & 0.003127 & -1.4 & $\mathrm{~N}$ & 1.824125 & -0.000079 & -1.599183 \\
\hline $\mathrm{H}$ & -0.218356 & -2.895742 & 0.65 & $\mathrm{H}$ & -1.3 & 290 & & $\mathrm{H}$ & 275 & 0074 & 1111 \\
\hline $\mathrm{H}$ & -0.991189 & -2.05 & & $\mathrm{H}$ & -2.4 & 687 & & $\mathrm{H}$ & 202 & 492 & 098 \\
\hline $\mathrm{N}$ & 1.68 & -1.1 & -1.3 & $\mathrm{~N}$ & -0.0 & 3863 & -0.2 & $\mathrm{~N}$ & 242 & 514 & 327 \\
\hline $\mathrm{H}$ & 2.38 & -1.6( & -0.7 & $\mathrm{H}$ & -0.8 & 515 & & $\mathrm{H}$ & 200 & 160 & 905 \\
\hline $\mathrm{H}$ & 2.193456 & -0.461426 & -1.90 & $\mathrm{H}$ & 0.796243 & -2.870457 & 15 & $\mathrm{H}$ & -0.786301 & 078 & 0.449668 \\
\hline $\mathrm{N}$ & 0.166385 & 2.045560 & -1.249592 & $\mathrm{~N}$ & 1.710308 & -0.002471 & 1.672937 & $\mathrm{~N}$ & -1.797619 & -0.000160 & 1.640052 \\
\hline $\mathrm{H}$ & 0.991188 & 2.057503 & -1.849340 & $\mathrm{H}$ & 2.312000 & -0.820416 & 1.575542 & $\mathrm{H}$ & -2.415058 & 0.811145 & 1.591562 \\
\hline $\mathrm{H}$ & -0.628256 & 2.173654 & -1.875827 & $\mathrm{H}$ & 2.315596 & 0.812585 & 1.573495 & $\mathrm{H}$ & -2.415952 & -0.810714 & 1.590369 \\
\hline $\mathrm{H}$ & 0.628256 & -2.173654 & & $\mathrm{H}$ & -2.49 & -0.8 & & $\mathrm{H}$ & & & 124 \\
\hline $\mathrm{H}$ & -2.3 & 1.6 & 0.7 & $\mathrm{H}$ & & & & $\mathrm{H}$ & 05 & 11 & 018 \\
\hline $\mathrm{H}$ & 0.2 & 2.8 & -0.6 & $\mathrm{H}$ & & $-0 .($ & & $\mathrm{H}$ & & 64 & 338 \\
\hline $\mathrm{H}$ & 1.27 & -1.7 & -1.9 & $\mathrm{H}$ & 0.0 & -2.5 & -1 & $\mathrm{H}$ & 731 & 29 & 967 \\
\hline $\mathrm{N}$ & -1.822006 & -0.836053 & -1.3 & $\mathrm{~N}$ & 1.88 & -0.002867 & 386 & $\mathrm{~N}$ & -1.825582 & 0.000814 & -1.598594 \\
\hline $\mathrm{H}$ & -1.522095 & -1.581371 & -1.949935 & $\mathrm{H}$ & 2.489693 & -0.829293 & -1.4 & $\mathrm{H}$ & -2.440652 & 0.812107 & -1.531622 \\
\hline $\mathrm{H}$ & -2.223402 & -0.116357 & -1.922632 & $\mathrm{H}$ & 2.491967 & 0.821906 & -1.415415 & $\mathrm{H}$ & 0540 & -0.810646 & -1.532622 \\
\hline $\mathrm{H}$ & -2.601463 & -1.219446 & -0.788674 & $\mathrm{H}$ & 1.379447 & -0.002140 & -2.306047 & $\mathrm{H}$ & -1.487402 & 0.001432 & -2.560698 \\
\hline $\mathrm{N}$ & 1.822006 & 0.8 & 66 & $\mathrm{~N}$ & 248 & 233 & & $\mathrm{~N}$ & 945 & $-0 .($ & 916 \\
\hline $\mathrm{H}$ & 2.601463 & 1.2 & 575 & $\mathrm{H}$ & 4637 & -0.0 & & $\mathrm{H}$ & 1038 & 226 & 2.594795 \\
\hline $\mathrm{H}$ & & 0.1 & & $\mathrm{H}$ & 2040 & & & $\mathrm{H}$ & & & \\
\hline $\mathrm{H}$ & 1.522094 & 1.5 & & $\mathrm{H}$ & 07752 & 0.820305 & & $\mathrm{H}$ & 608 & 843 & 314 \\
\hline \multicolumn{12}{|c|}{$\mathrm{Sc}\left(\mathrm{NH}_{3}\right)_{7}^{+}$} \\
\hline $\mathrm{Sc}$ & -0.005352 & 650 & 622 & $\mathrm{Sc}$ & 0.010748 & 0.000458 & 0.004109 & & & N/A & \\
\hline $\mathrm{N}$ & 0.480301 & 0.070120 & -2.382503 & $\mathrm{~N}$ & 0.830038 & 0.849149 & -2.089083 & & & & \\
\hline $\mathrm{H}$ & 0.997564 & -0.727715 & -2.749493 & $\mathrm{H}$ & 1.764343 & 1.257888 & -2.106786 & & & & \\
\hline $\mathrm{H}$ & 0.980350 & 0.897124 & -2.706164 & $\mathrm{H}$ & 0.246323 & 1.404431 & -2.713391 & & & & \\
\hline $\mathrm{N}$ & -0.975136 & 2.208979 & -0.620655 & $\mathrm{~N}$ & -1.812735 & -0.811877 & -1.35 & & & & \\
\hline $\mathrm{H}$ & -1.784703 & 2.089940 & -1.229247 & $\mathrm{H}$ & -1.425793 & -1.548470 & -1.952832 & & & & \\
\hline $\mathrm{H}$ & -0.333721 & 2.804466 & -1.141123 & $\mathrm{H}$ & -2.313458 & -0.169720 & -1.971952 & & & & \\
\hline $\mathrm{N}$ & 0.077017 & -0.077453 & 2.472330 & $\mathrm{~N}$ & -1.175484 & -0.349913 & 2.054177 & & & & \\
\hline
\end{tabular}




\begin{tabular}{|c|c|c|c|c|c|c|c|c|}
\hline & & & & & & & & \\
\hline $\mathrm{H}$ & 1.014941 & -0.070483 & 2.865971 & $\mathrm{H}$ & -1.577519 & 0.410295 & 2.599828 & \\
\hline $\mathrm{H}$ & -0.370029 & -0.911780 & 2.845067 & $\mathrm{H}$ & -0.351003 & -0.696882 & 2.554219 & \\
\hline $\mathrm{N}$ & 1.910192 & -1.518658 & 0.271234 & $\mathrm{~N}$ & 1.230503 & 1.646264 & 1.288116 & \\
\hline $\mathrm{H}$ & 1.581487 & -2.442770 & 0.540444 & $\mathrm{H}$ & 1.982798 & 1.240428 & 1.842488 & \\
\hline $\mathrm{H}$ & 2.425462 & -1.644551 & -0.598196 & $\mathrm{H}$ & 1.657469 & 2.296151 & 0.625656 & \\
\hline $\mathrm{H}$ & -1.301078 & 2.768512 & 0.165468 & $\mathrm{H}$ & -2.509311 & -1.256962 & -0.755878 & \\
\hline $\mathrm{H}$ & -0.406583 & 0.074058 & -2.888493 & $\mathrm{H}$ & 0.917229 & -0.088297 & -2.498725 & \\
\hline $\mathrm{H}$ & 2.604944 & -1.261012 & 0.968293 & $\mathrm{H}$ & 0.664196 & 2.202490 & 1.925880 & \\
\hline $\mathrm{H}$ & -0.402724 & 0.714000 & 2.894572 & $\mathrm{H}$ & -1.854089 & -1.111301 & 2.045672 & \\
\hline $\mathrm{N}$ & -2.404718 & -0.043974 & 0.636199 & $\mathrm{~N}$ & 0.000709 & -2.440217 & 0.250508 & \\
\hline $\mathrm{H}$ & -3.023711 & -0.032451 & -0.174190 & $\mathrm{H}$ & 0.426287 & -2.700448 & -0.645727 & \\
\hline $\mathrm{H}$ & -2.682989 & 0.752047 & 1.206640 & $\mathrm{H}$ & -0.876139 & -2.961741 & 0.318226 & \\
\hline $\mathrm{H}$ & -2.661516 & -0.873845 & 1.167085 & $\mathrm{H}$ & 0.606898 & -2.793770 & 0.994110 & \\
\hline $\mathrm{N}$ & 1.865041 & 1.557388 & 0.364016 & $\mathrm{~N}$ & -1.484706 & 1.923566 & -0.100801 & \\
\hline $\mathrm{H}$ & 1.508389 & 2.459065 & 0.670938 & $\mathrm{H}$ & -2.423215 & 1.546933 & 0.024323 & \\
\hline $\mathrm{H}$ & 2.387266 & 1.736762 & -0.491739 & $\mathrm{H}$ & -1.462803 & 2.365700 & -1.018293 & \\
\hline $\mathrm{H}$ & 2.557950 & 1.286426 & 1.057873 & $\mathrm{H}$ & -1.384278 & 2.670414 & 0.584286 & \\
\hline $\mathrm{N}$ & -0.917967 & -2.197639 & -0.728983 & $\mathrm{~N}$ & 2.354734 & -0.731901 & -0.041964 & \\
\hline $\mathrm{H}$ & -1.228480 & -2.804628 & 0.027651 & $\mathrm{H}$ & 2.431044 & -1.127645 & 0.899297 & \\
\hline $\mathrm{H}$ & -1.731115 & -2.068607 & -1.330770 & $\mathrm{H}$ & 2.537027 & -1.484418 & -0.709431 & \\
\hline $\mathrm{H}$ & -0.262430 & -2.749561 & -1.279110 & $\mathrm{H}$ & 3.116877 & -0.060186 & -0.144957 & \\
\hline \multicolumn{9}{|c|}{$\mathrm{Sc}\left(\mathrm{NH}_{3}\right){ }_{8}^{+}$} \\
\hline $\mathrm{H}$ & 0.682228 & -2.748655 & 1.244091 & $\mathrm{H}$ & 0.161985 & -2.720312 & -1.297438 & \multirow[t]{33}{*}{$\mathrm{N} / \mathrm{A}$} \\
\hline $\mathrm{N}$ & -0.099155 & -2.113401 & 1.388169 & $\mathrm{H}$ & -1.452319 & -2.466023 & -1.309577 & \\
\hline $\mathrm{H}$ & -1.472838 & -2.424266 & -1.237594 & $\mathrm{~N}$ & -0.550881 & -1.998440 & -1.380120 & \\
\hline $\mathrm{H}$ & -0.936187 & -2.673150 & 1.243454 & $\mathrm{H}$ & 2.499572 & -1.373343 & -0.835219 & \\
\hline $\mathrm{H}$ & -0.088934 & -1.885449 & 2.380628 & $\mathrm{H}$ & -0.481096 & -1.608633 & -2.318506 & \\
\hline $\mathrm{H}$ & 1.215443 & -2.552298 & -1.254328 & $\mathrm{~N}$ & 2.043755 & -0.614212 & -1.340375 & \\
\hline $\mathrm{H}$ & 1.276093 & -1.385980 & -2.380104 & $\mathrm{H}$ & 1.986197 & -0.904013 & -2.315625 & \\
\hline $\mathrm{N}$ & -1.565439 & -1.422410 & -1.388111 & $\mathrm{H}$ & 2.689709 & 0.172863 & -1.310609 & \\
\hline $\mathrm{N}$ & 1.422379 & -1.565314 & -1.388343 & $\mathrm{Sc}$ & -0.000002 & -0.000001 & -0.031935 & \\
\hline $\mathrm{H}$ & -2.749181 & -0.681993 & 1.244426 & $\mathrm{H}$ & -2.689877 & -0.172870 & -1.310272 & \\
\hline $\mathrm{H}$ & -2.552280 & -1.216145 & -1.252043 & $\mathrm{~N}$ & -2.043921 & 0.614199 & -1.340130 & \\
\hline $\mathrm{H}$ & -1.388269 & -1.275973 & -2.380245 & $\mathrm{H}$ & -1.986488 & 0.903991 & -2.315389 & \\
\hline $\mathrm{H}$ & 2.424288 & -1.473717 & -1.237579 & $\mathrm{H}$ & 0.480810 & 1.608604 & -2.318585 & \\
\hline $\mathrm{Sc}$ & -0.000000 & 0.000001 & 0.000567 & $\mathrm{~N}$ & 0.550711 & 1.998422 & -1.380211 & \\
\hline $\mathrm{N}$ & -2.113636 & 0.099219 & 1.388185 & $\mathrm{H}$ & -2.499663 & 1.373338 & -0.834919 & \\
\hline $\mathrm{H}$ & 2.673340 & -0.936458 & 1.243149 & $\mathrm{H}$ & 1.452156 & 2.466007 & -1.309785 & \\
\hline $\mathrm{H}$ & -1.885406 & 0.089071 & 2.380579 & $\mathrm{H}$ & -0.162145 & 2.720295 & -1.297450 & \\
\hline $\mathrm{N}$ & 2.113799 & -0.099297 & 1.387931 & $\mathrm{H}$ & 0.350560 & -2.680509 & 1.380624 & \\
\hline $\mathrm{H}$ & -2.673203 & 0.936382 & 1.243513 & $\mathrm{H}$ & 1.863882 & -2.064037 & 1.411539 & \\
\hline $\mathrm{H}$ & 1.885688 & -0.089198 & 2.380353 & $\mathrm{~N}$ & 0.875846 & -1.805819 & 1.419626 & \\
\hline $\mathrm{H}$ & 0.089182 & 1.885318 & 2.380720 & $\mathrm{H}$ & -2.076846 & -1.915268 & 1.143615 & \\
\hline $\mathrm{H}$ & 2.749337 & 0.681916 & 1.244138 & $\mathrm{H}$ & 0.696442 & -1.405874 & 2.349075 & \\
\hline $\mathrm{N}$ & 0.099318 & 2.113324 & 1.388273 & $\mathrm{~N}$ & -1.890245 & -0.922782 & 1.293554 & \\
\hline $\mathrm{H}$ & -0.682062 & 2.748607 & 1.244303 & $\mathrm{H}$ & -1.614848 & -0.829879 & 2.277953 & \\
\hline $\mathrm{N}$ & 1.565273 & 1.422488 & -1.388216 & $\mathrm{H}$ & -2.788515 & -0.444158 & 1.210674 & \\
\hline $\mathrm{H}$ & 1.387978 & 1.276109 & -2.380337 & $\mathrm{H}$ & 2.788661 & 0.444168 & 1.210329 & \\
\hline $\mathrm{H}$ & 1.472695 & 2.424335 & -1.237628 & $\mathrm{~N}$ & 1.890403 & 0.922797 & 1.293312 & \\
\hline $\mathrm{H}$ & 0.936352 & 2.673060 & 1.243513 & $\mathrm{H}$ & 1.615126 & 0.829911 & 2.277746 & \\
\hline $\mathrm{H}$ & 2.552130 & 1.216210 & -1.252283 & $\mathrm{H}$ & -0.696150 & 1.405901 & 2.349146 & \\
\hline $\mathrm{N}$ & -1.422540 & 1.565390 & -1.388096 & $\mathrm{~N}$ & -0.875671 & 1.805835 & 1.419714 & \\
\hline $\mathrm{H}$ & -2.424432 & 1.473803 & -1.237210 & $\mathrm{H}$ & 2.076990 & 1.915281 & 1.143334 & \\
\hline $\mathrm{H}$ & -1.276381 & 1.386090 & -2.379883 & $\mathrm{H}$ & -1.863706 & 2.064056 & 1.411749 & \\
\hline $\mathrm{H}$ & -1.215573 & 2.552366 & -1.254071 & $\mathrm{H}$ & -0.350386 & 2.680523 & 1.380634 & \\
\hline
\end{tabular}


Table S16: Harmonic vibrational frequencies $\left(\mathrm{cm}^{-1}\right)$ for the $\mathrm{Sc}\left(\mathrm{NH}_{3}\right)_{\mathrm{n}=1-8}{ }^{+}$species (singlet spin multiplicity) optimized at CAM-B3LYP, MN15, and MP2 combined with the cc-pVTZ(Sc,N) augcc-pVTZ $(\mathrm{H})$ basis sets.

\begin{tabular}{|c|c|c|}
\hline CAM-B3LYP & MN15 & MP2 \\
\hline \multicolumn{3}{|c|}{$\mathrm{Sc}\left(\mathrm{NH}_{3}\right)_{1}{ }^{+}$} \\
\hline 408 & 404 & 412 \\
\hline 534 & 507 & 540 \\
\hline 535 & 508 & 540 \\
\hline 1294 & 1257 & 1302 \\
\hline 1635 & 1596 & 1638 \\
\hline 1635 & 1596 & 1638 \\
\hline 3426 & 3424 & 3419 \\
\hline 3512 & 3520 & 3534 \\
\hline 3512 & 3520 & 3534 \\
\hline \multicolumn{3}{|c|}{$\mathrm{Sc}\left(\mathrm{NH}_{3}\right)_{2}^{+}$} \\
\hline $6 i$ & 16 & $16 i$ \\
\hline 51 & 54 & 73 \\
\hline 51 & 54 & 73 \\
\hline 341 & 340 & 345 \\
\hline 406 & 405 & 413 \\
\hline 490 & 470 & 496 \\
\hline 490 & 470 & 496 \\
\hline 497 & 473 & 502 \\
\hline 497 & 473 & 503 \\
\hline 1271 & 1233 & 1277 \\
\hline 1276 & 1239 & 1282 \\
\hline 1640 & 1600 & 1641 \\
\hline 1640 & 1600 & 1641 \\
\hline 1643 & 1603 & 1643 \\
\hline 1643 & 1603 & 1643 \\
\hline 3437 & 3434 & 3428 \\
\hline 3437 & 3434 & 3429 \\
\hline 3528 & 3535 & 3547 \\
\hline 3528 & 3535 & 3547 \\
\hline 3531 & 3538 & 3549 \\
\hline 3531 & 3538 & 3549 \\
\hline \multicolumn{3}{|c|}{$\mathrm{Sc}\left(\mathrm{NH}_{3}\right)_{3}{ }^{+}$} \\
\hline 7 & 23 & $5 i$ \\
\hline 77 & 83 & 80 \\
\hline 97 & 96 & 98 \\
\hline 101 & 99 & 103 \\
\hline 126 & 145 & 139 \\
\hline 127 & 156 & 148 \\
\hline 312 & 300 & 312 \\
\hline 313 & 309 & 317 \\
\hline 393 & 393 & 405 \\
\hline 447 & 424 & 451 \\
\hline 451 & 430 & 461 \\
\hline 458 & 439 & 463 \\
\hline 468 & 442 & 469 \\
\hline 489 & 469 & 485 \\
\hline 546 & 534 & 562 \\
\hline 1240 & 1200 & 1248 \\
\hline
\end{tabular}




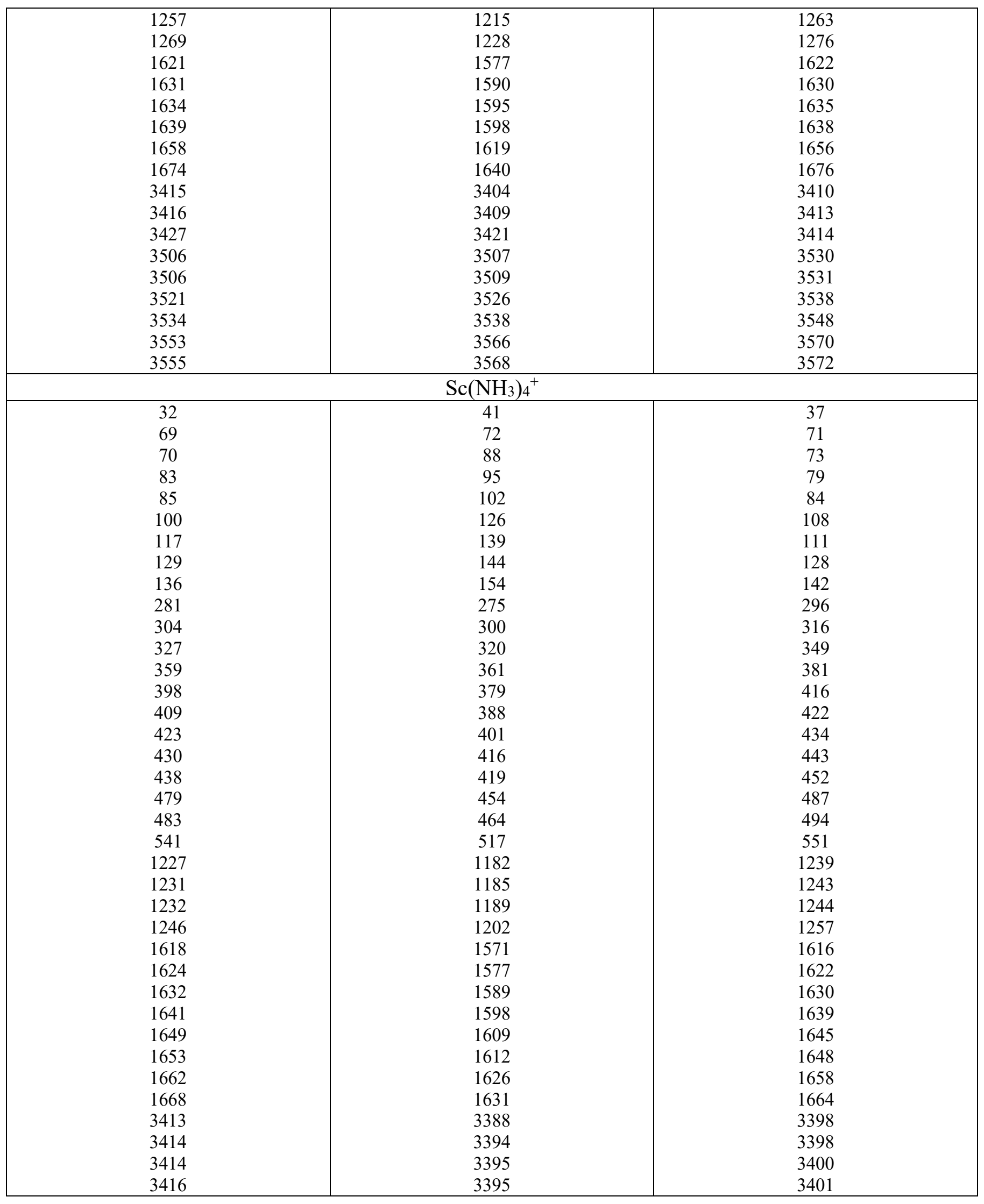




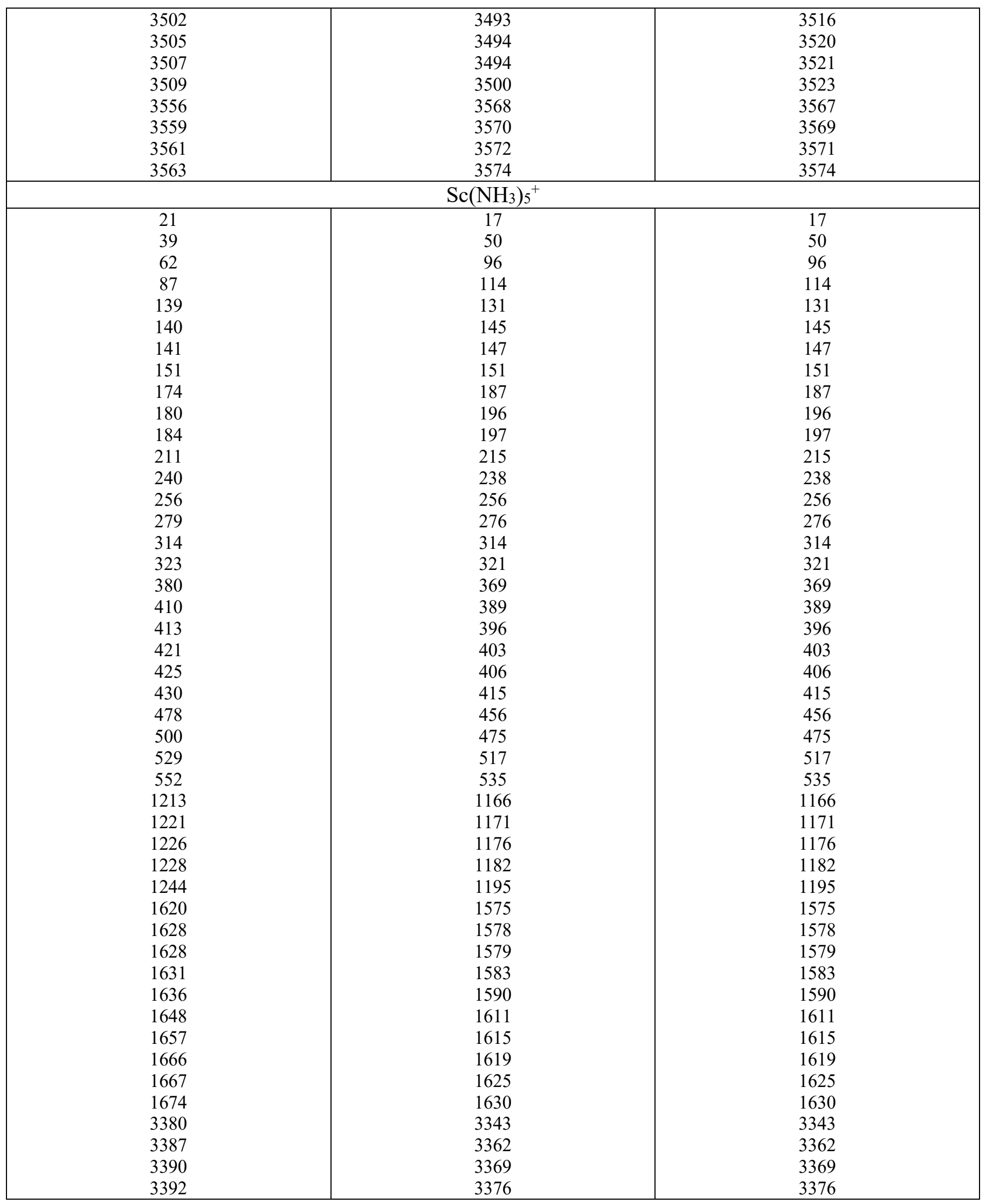




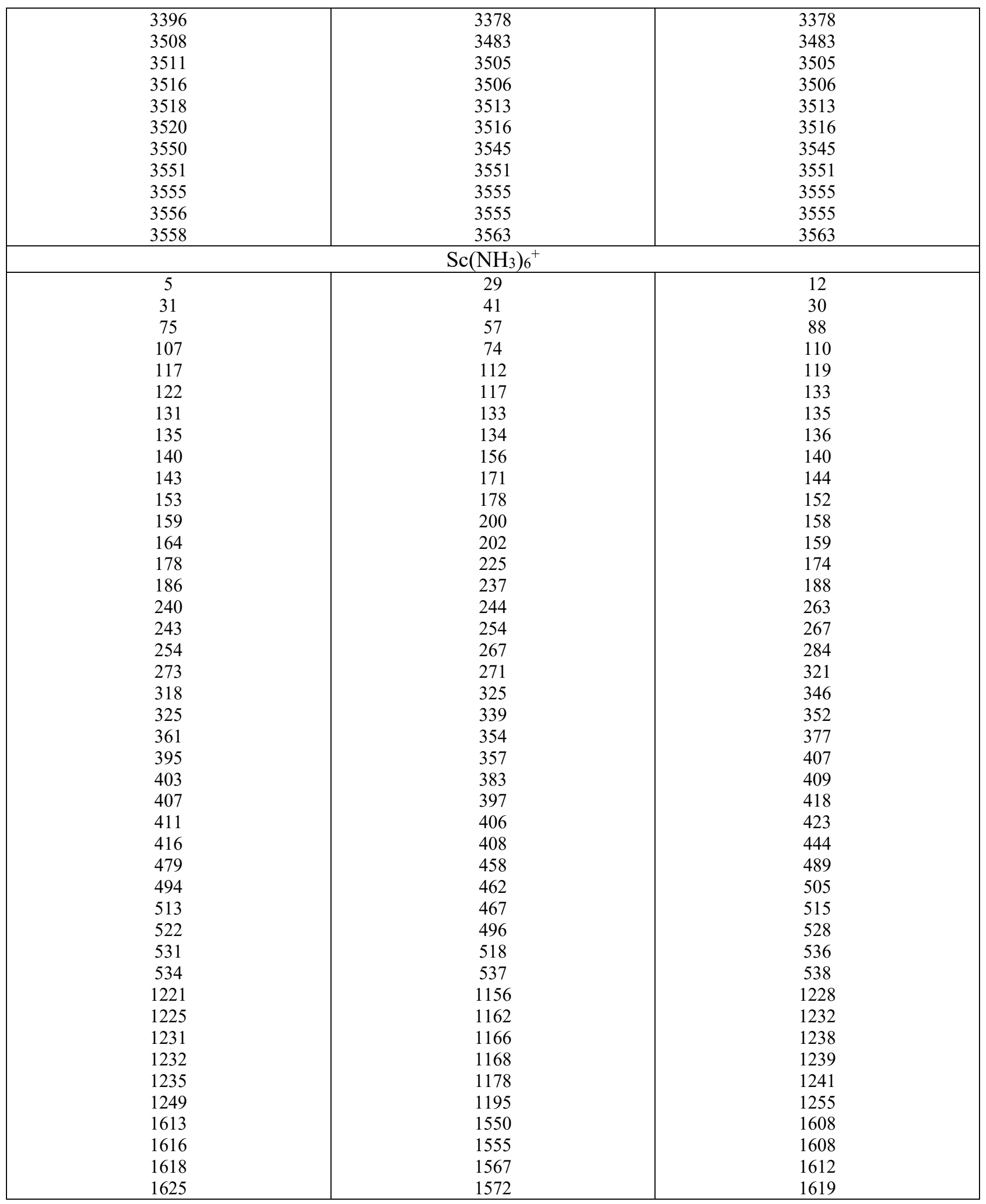




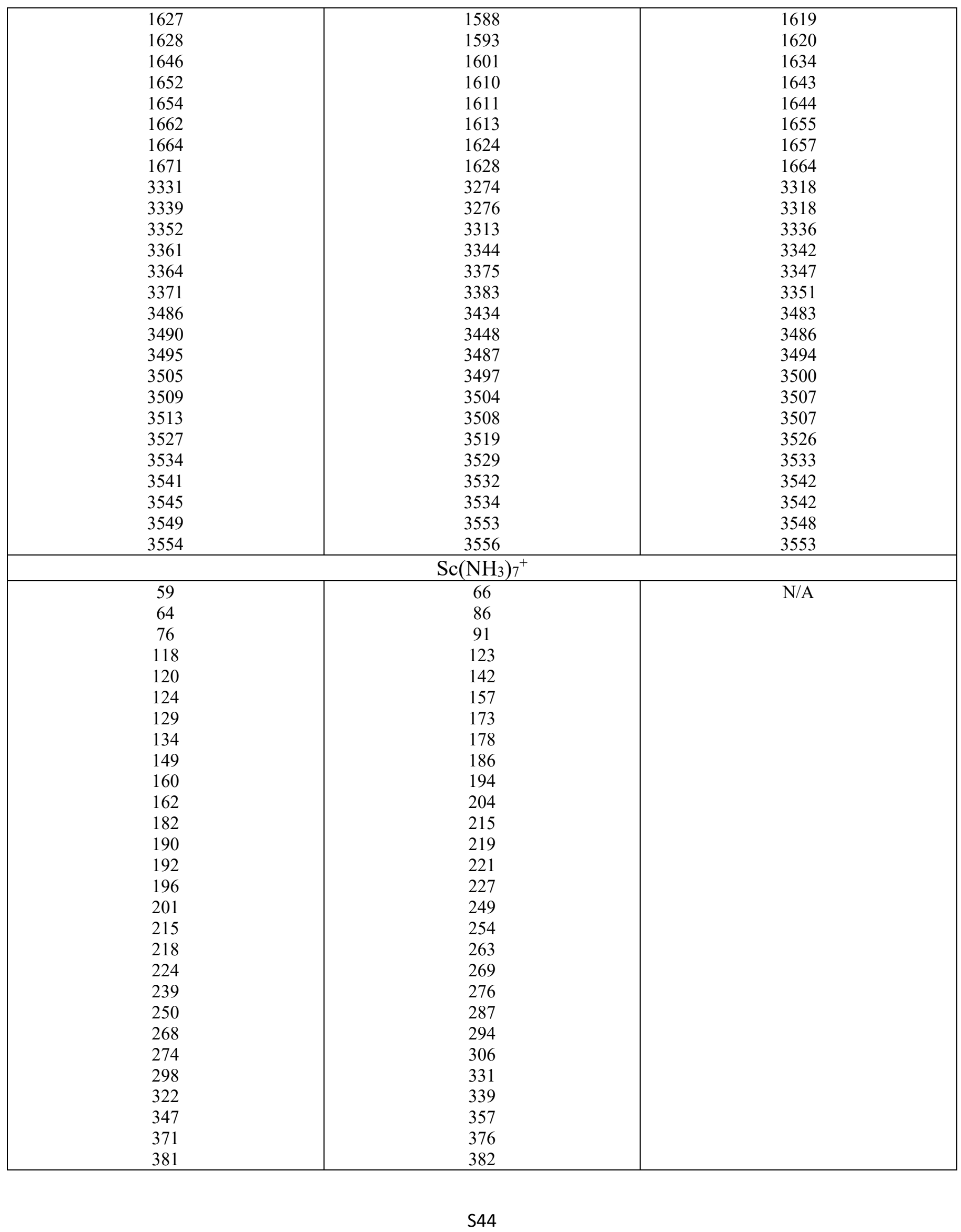




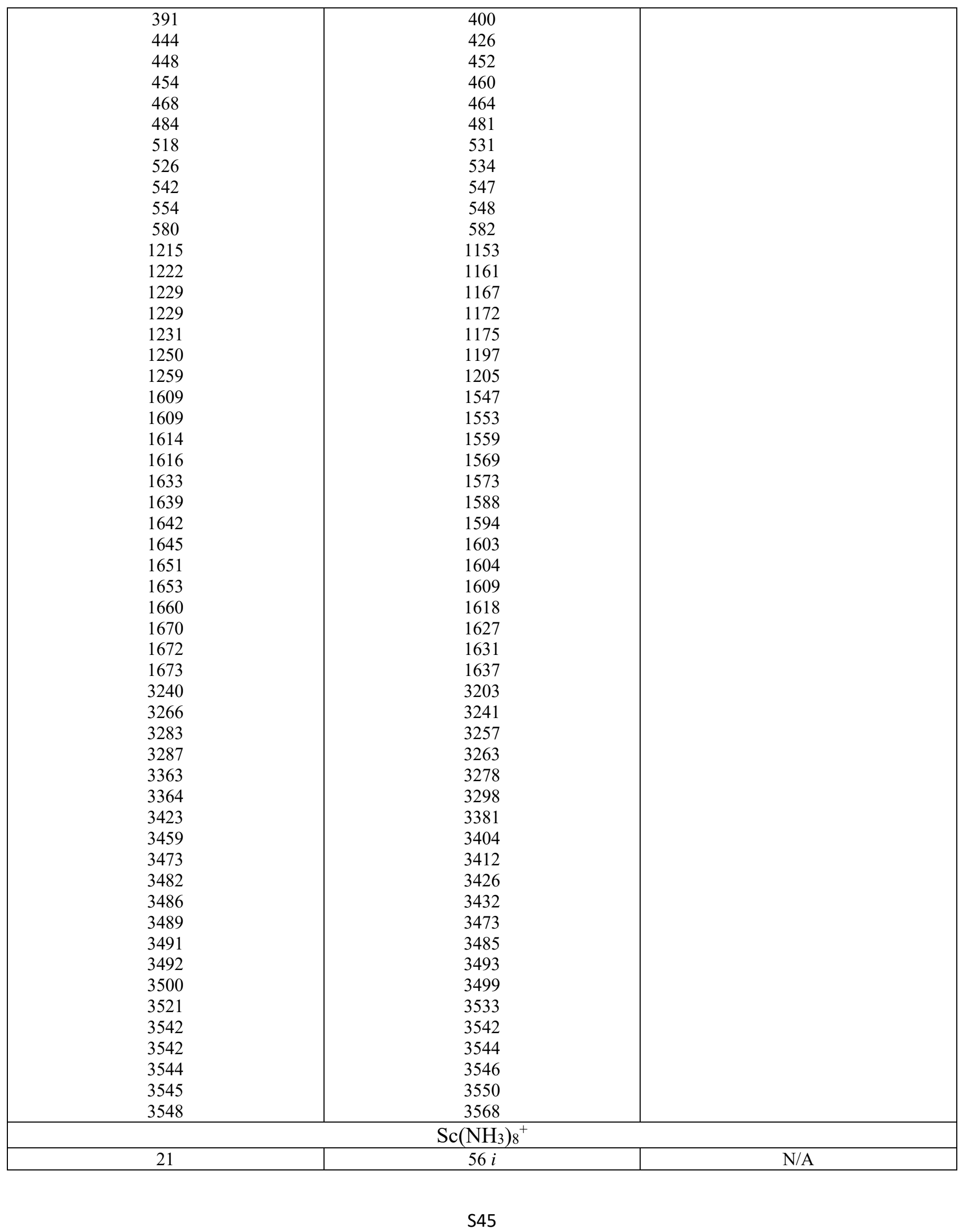




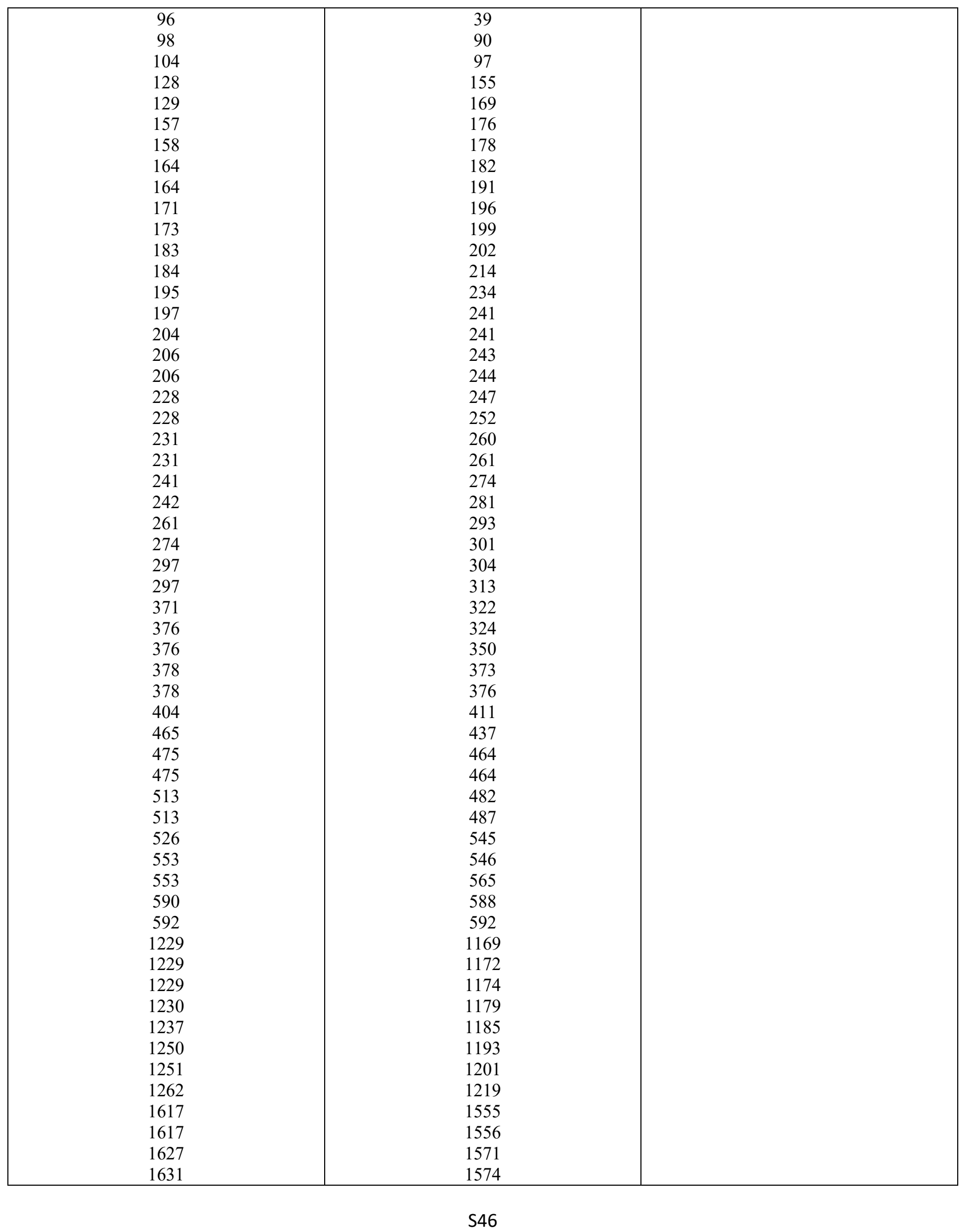




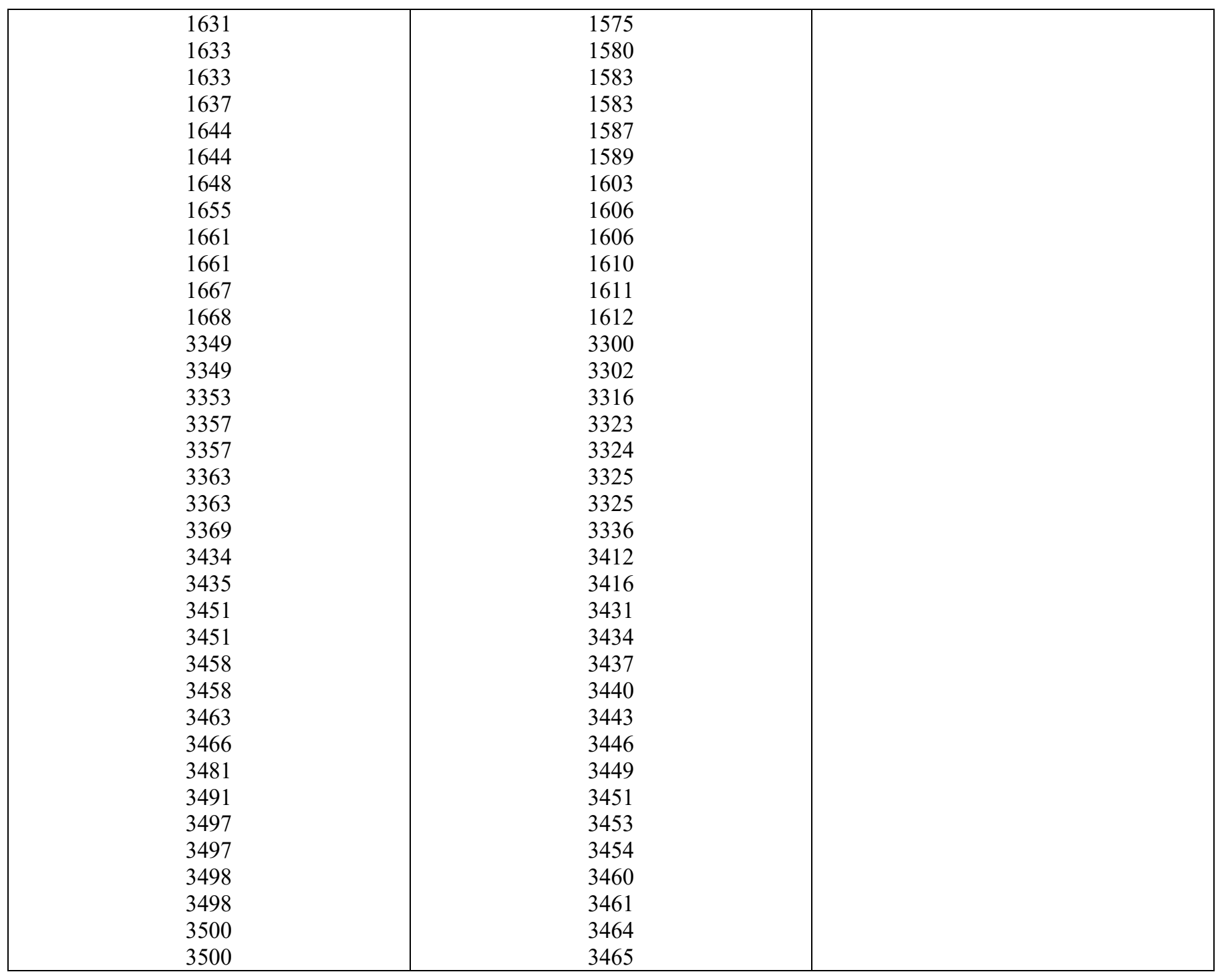


Table S17: Harmonic vibrational frequencies $\left(\mathrm{cm}^{-1}\right)$ for the $\mathrm{Sc}\left(\mathrm{NH}_{3}\right)_{\mathrm{n}=1-8^{+}}$species (triplet spin multiplicity) optimized at CAM-B3LYP, MN15, and MP2 combined with the cc-pVTZ(Sc,N) augcc-pVTZ $(\mathrm{H})$ basis sets.

\begin{tabular}{|c|c|c|}
\hline CAM-B3LYP & MN15 & MP2 \\
\hline \multicolumn{3}{|c|}{$\mathrm{Sc}\left(\mathrm{NH}_{3}\right)_{1}{ }^{+}$} \\
\hline 371 & 356 & 339 \\
\hline 518 & 539 & 510 \\
\hline 532 & 654 & 511 \\
\hline 1303 & 1271 & 1310 \\
\hline 1649 & 1617 & 1657 \\
\hline 1672 & 1760 & 1657 \\
\hline 3441 & 3450 & 3443 \\
\hline 3530 & 3550 & 3557 \\
\hline 3542 & 3551 & 3558 \\
\hline \multicolumn{3}{|c|}{$\mathrm{Sc}\left(\mathrm{NH}_{3}\right)_{2}^{+}$} \\
\hline 5 & 31 & $15 i$ \\
\hline 54 & 77 & 34 \\
\hline 58 & 80 & 97 \\
\hline 305 & 293 & 211 \\
\hline 371 & 358 & 277 \\
\hline 477 & 457 & 346 \\
\hline 483 & 477 & 434 \\
\hline 483 & 495 & 487 \\
\hline 486 & 514 & 497 \\
\hline 1273 & 1241 & 1279 \\
\hline 1277 & 1246 & 1282 \\
\hline 1652 & 1596 & 1599 \\
\hline 1653 & 1618 & 1660 \\
\hline 1654 & 1625 & 1664 \\
\hline 1663 & 1628 & 1664 \\
\hline 3450 & 3453 & 3448 \\
\hline 3451 & 3454 & 3449 \\
\hline 3543 & 3550 & 3552 \\
\hline 3544 & 3550 & 3564 \\
\hline 3545 & 3562 & 3565 \\
\hline 3548 & 3563 & 3572 \\
\hline \multicolumn{3}{|c|}{$\mathrm{Sc}\left(\mathrm{NH}_{3}\right)_{3}{ }^{+}$} \\
\hline 7 & 7 & 11 \\
\hline 59 & 59 & 41 \\
\hline 85 & 85 & 88 \\
\hline 93 & 93 & 89 \\
\hline 114 & 114 & 117 \\
\hline 126 & 126 & 121 \\
\hline 276 & 275 & 267 \\
\hline 282 & 281 & 273 \\
\hline 360 & 360 & 355 \\
\hline 423 & 423 & 396 \\
\hline 437 & 437 & 415 \\
\hline 441 & 441 & 435 \\
\hline 444 & 444 & 439 \\
\hline 458 & 458 & 453 \\
\hline 523 & 523 & 523 \\
\hline 1249 & 1249 & 1256 \\
\hline
\end{tabular}




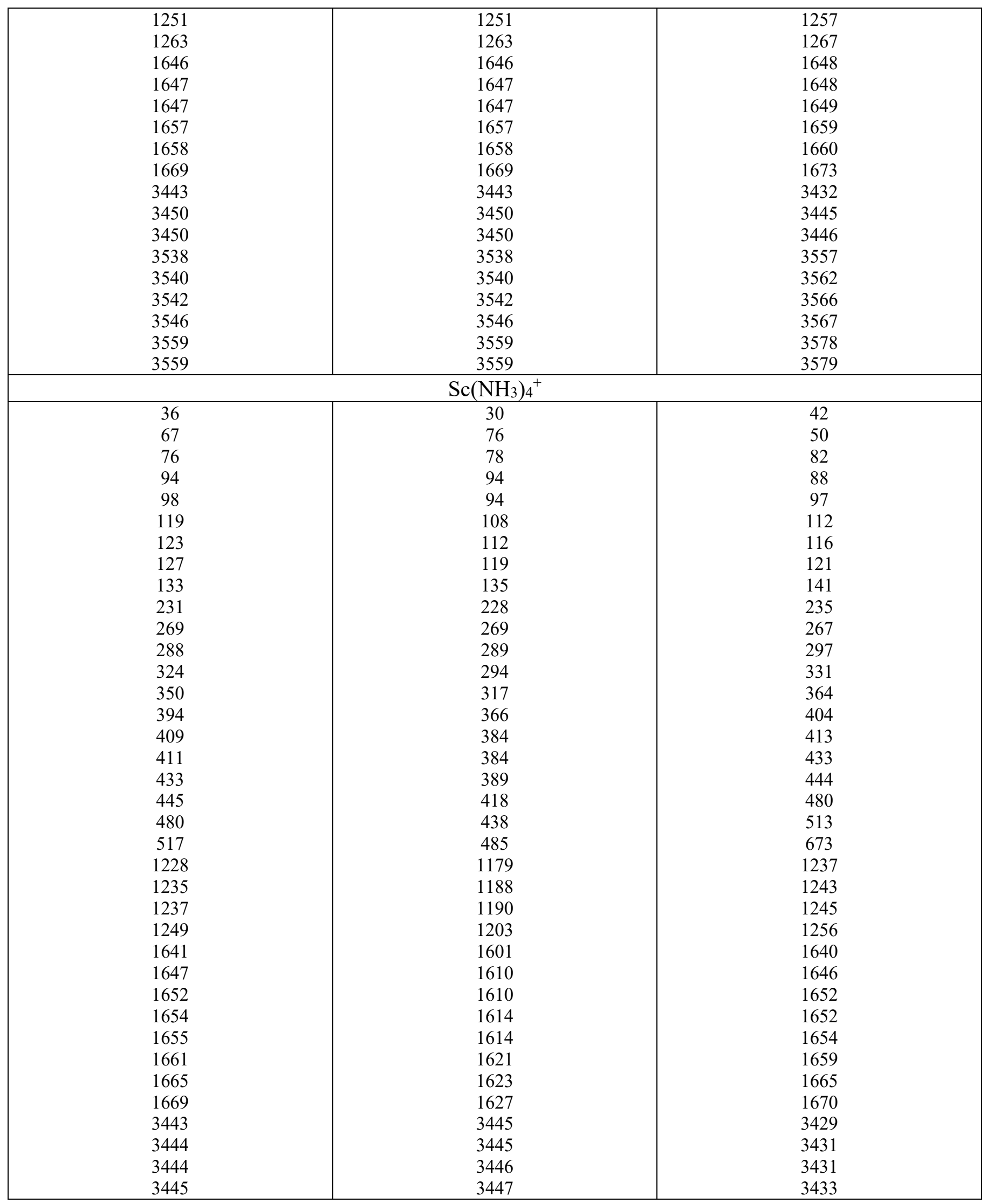




\begin{tabular}{|c|c|c|}
\hline $\begin{array}{l}3534 \\
3534 \\
3536 \\
3536 \\
3565 \\
3566 \\
3566 \\
3567 \\
\end{array}$ & $\begin{array}{l}3548 \\
3549 \\
3550 \\
3551 \\
3575 \\
3576 \\
3576 \\
3577 \\
\end{array}$ & $\begin{array}{l}3552 \\
3554 \\
3554 \\
3554 \\
3577 \\
3578 \\
3579 \\
3580 \\
\end{array}$ \\
\hline \multicolumn{3}{|c|}{$\mathrm{Sc}\left(\mathrm{NH}_{3}\right) 5^{+}$} \\
\hline 21 & 11 & 29 \\
\hline 45 & 45 & 49 \\
\hline 51 & 64 & 52 \\
\hline 62 & 66 & 69 \\
\hline 84 & 91 & 79 \\
\hline 100 & 103 & 83 \\
\hline 101 & 108 & 93 \\
\hline 103 & 110 & 97 \\
\hline 118 & 114 & 103 \\
\hline 119 & 118 & 104 \\
\hline 132 & 142 & 119 \\
\hline 134 & 146 & 122 \\
\hline 232 & 228 & 228 \\
\hline 240 & 259 & 255 \\
\hline 272 & 267 & 259 \\
\hline 288 & 275 & 290 \\
\hline 320 & 299 & 316 \\
\hline 357 & 305 & 361 \\
\hline 376 & 324 & 373 \\
\hline 386 & 326 & 376 \\
\hline 409 & 354 & 408 \\
\hline 442 & 400 & 438 \\
\hline 453 & 416 & 451 \\
\hline 454 & 417 & 461 \\
\hline 467 & 443 & 477 \\
\hline 496 & 452 & 502 \\
\hline 510 & 471 & 502 \\
\hline 1225 & 1164 & 1233 \\
\hline 1227 & 1170 & 1237 \\
\hline 1232 & 1177 & 1238 \\
\hline 1233 & 1179 & 1239 \\
\hline 1243 & 1187 & 1250 \\
\hline 1630 & 1586 & 1635 \\
\hline 1637 & 1593 & 1642 \\
\hline 1640 & 1595 & 1644 \\
\hline 1644 & 1600 & 1648 \\
\hline 1648 & 1603 & 1651 \\
\hline 1650 & 1605 & 1655 \\
\hline 1659 & 1613 & 1664 \\
\hline 1663 & 1617 & 1667 \\
\hline 1665 & 1623 & 1669 \\
\hline 1668 & 1626 & 1674 \\
\hline 3410 & 3385 & 3421 \\
\hline 3411 & 3390 & 3422 \\
\hline 3416 & 3400 & 3426 \\
\hline 3419 & 3408 & 3427 \\
\hline
\end{tabular}




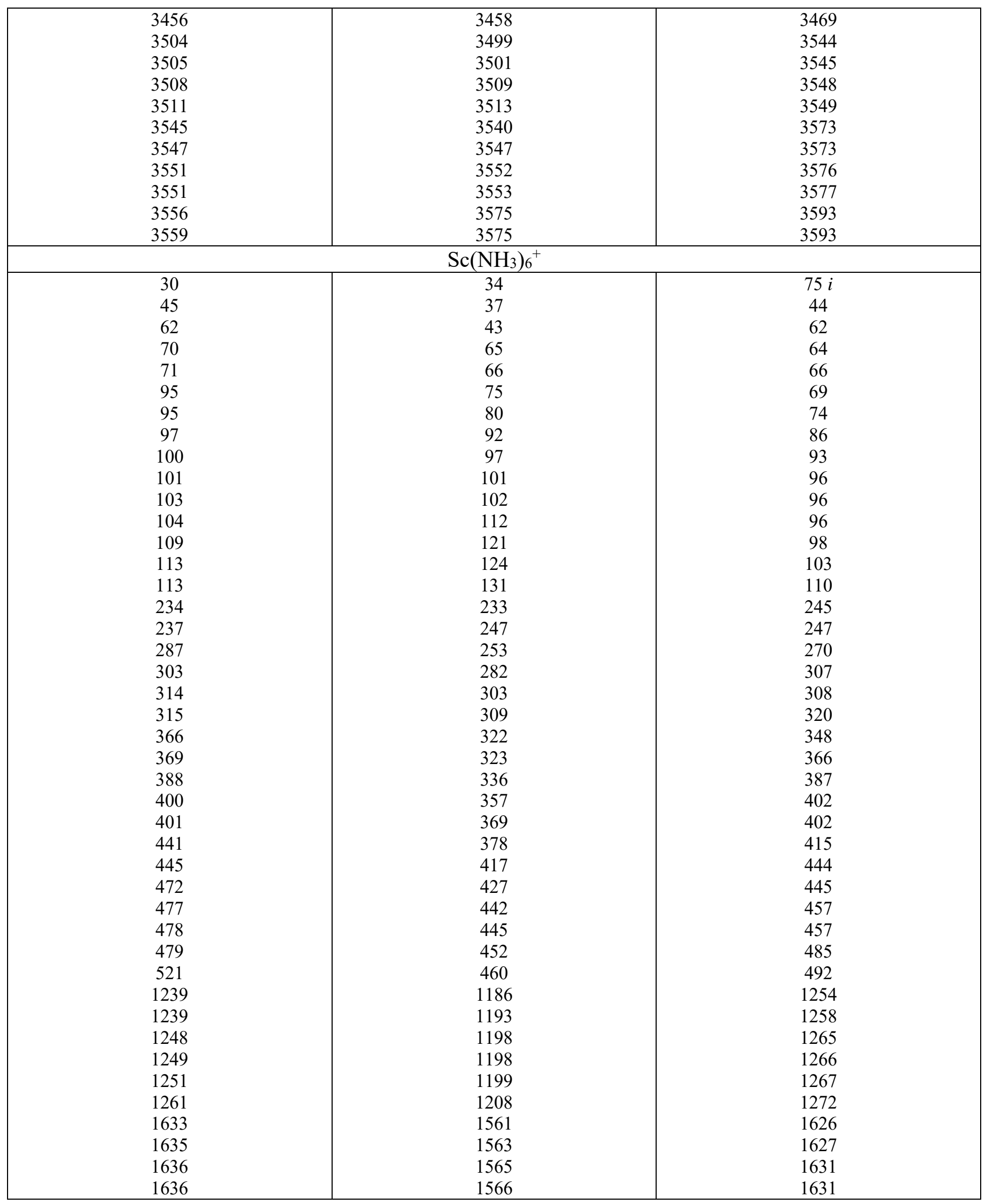




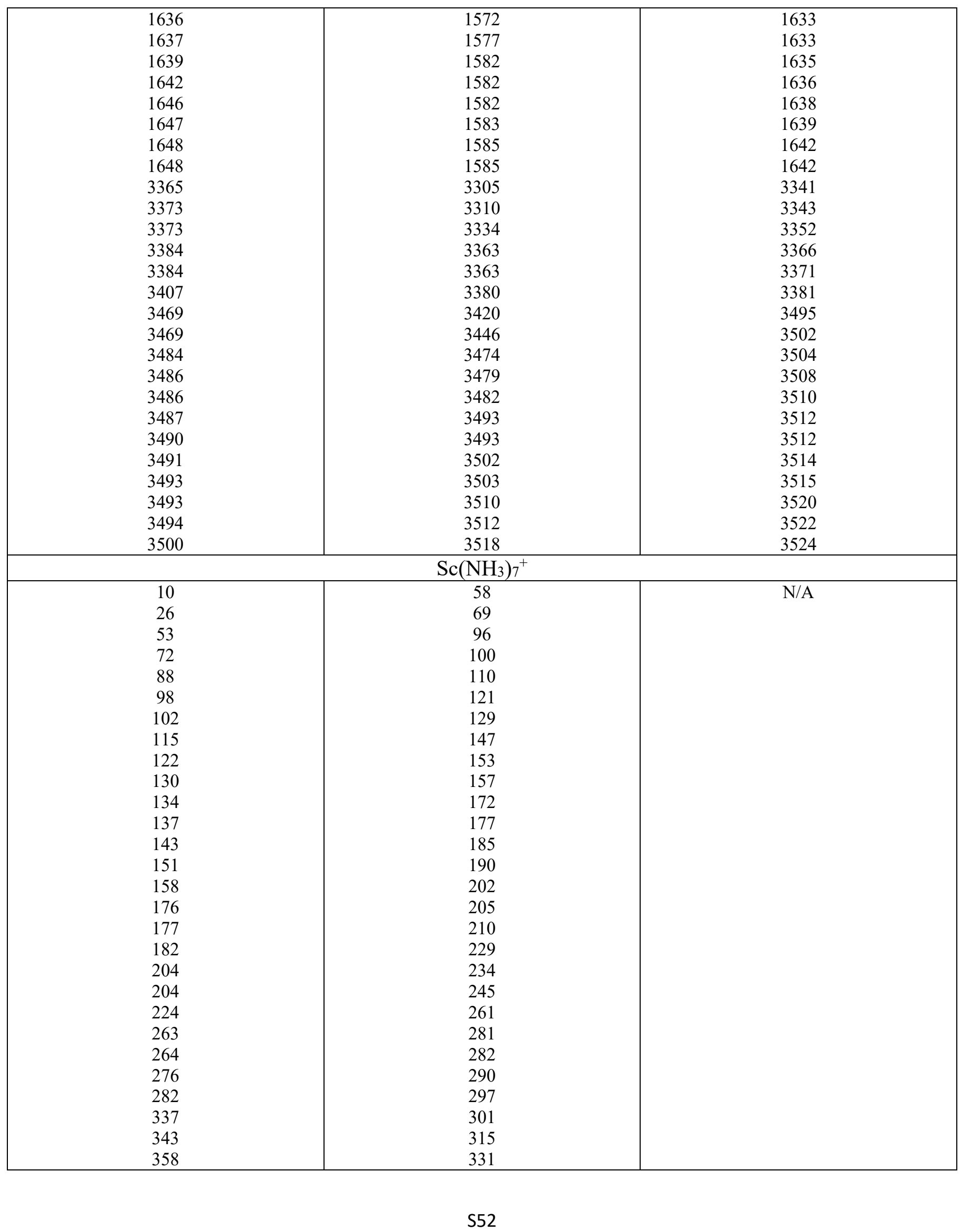




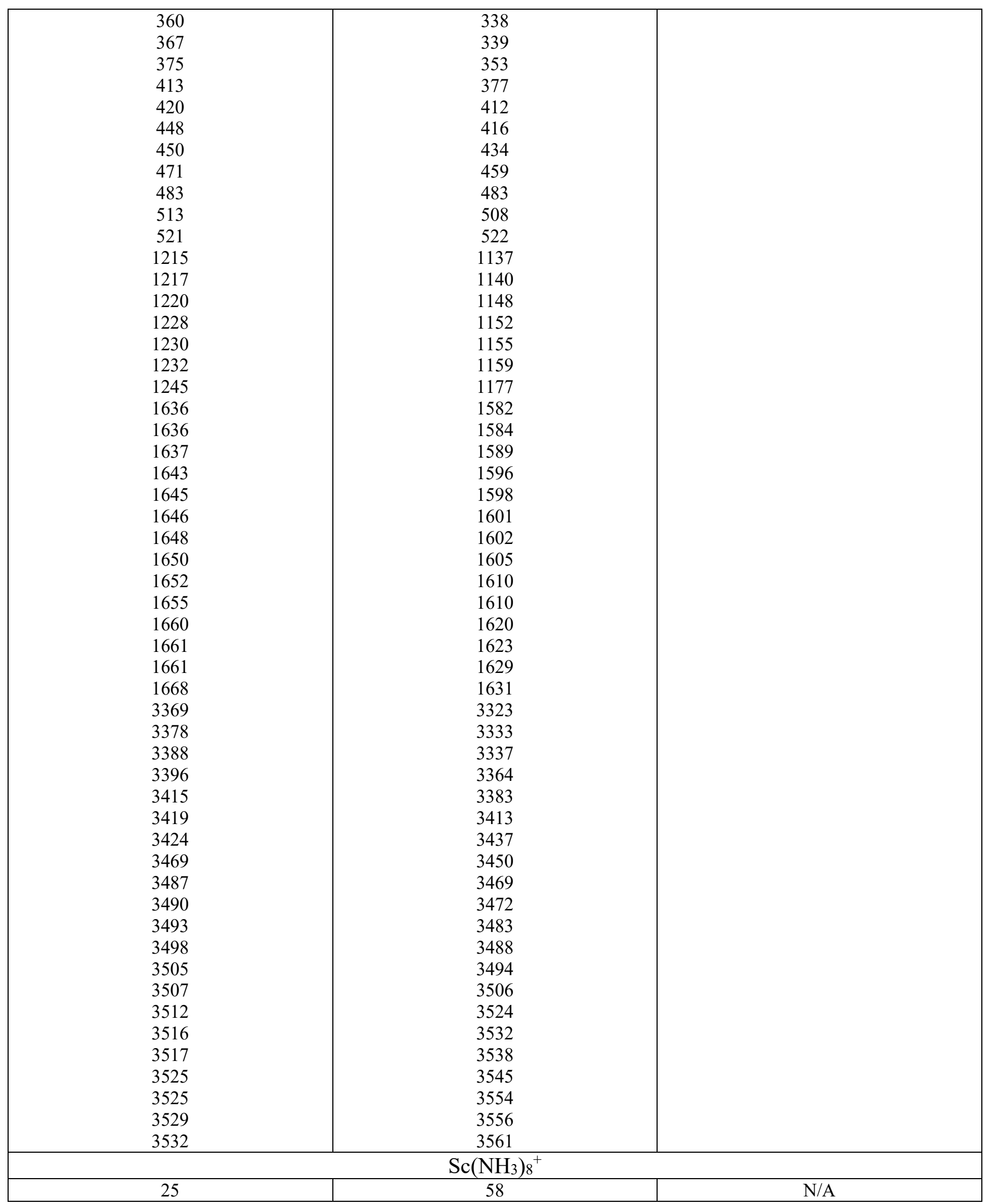




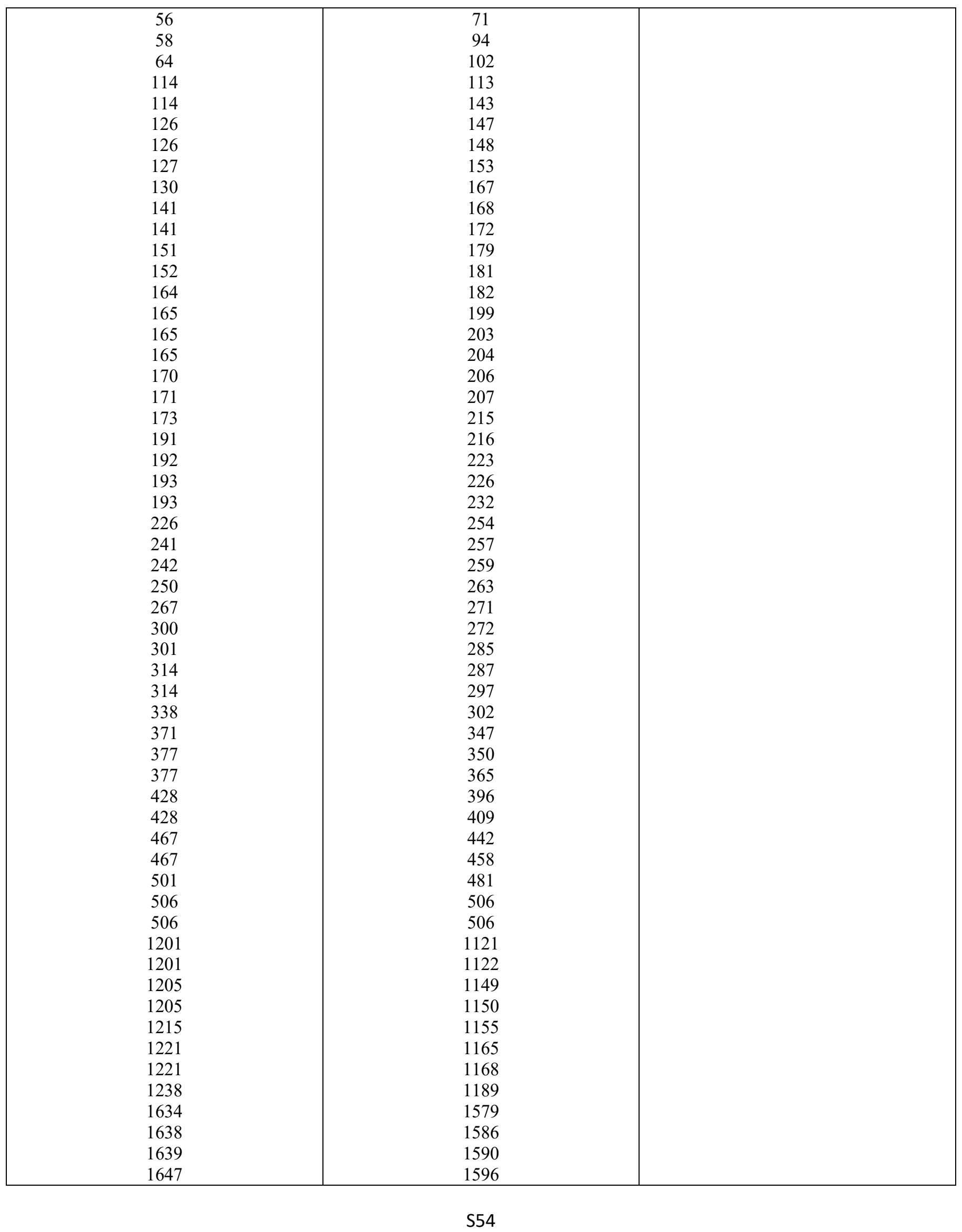




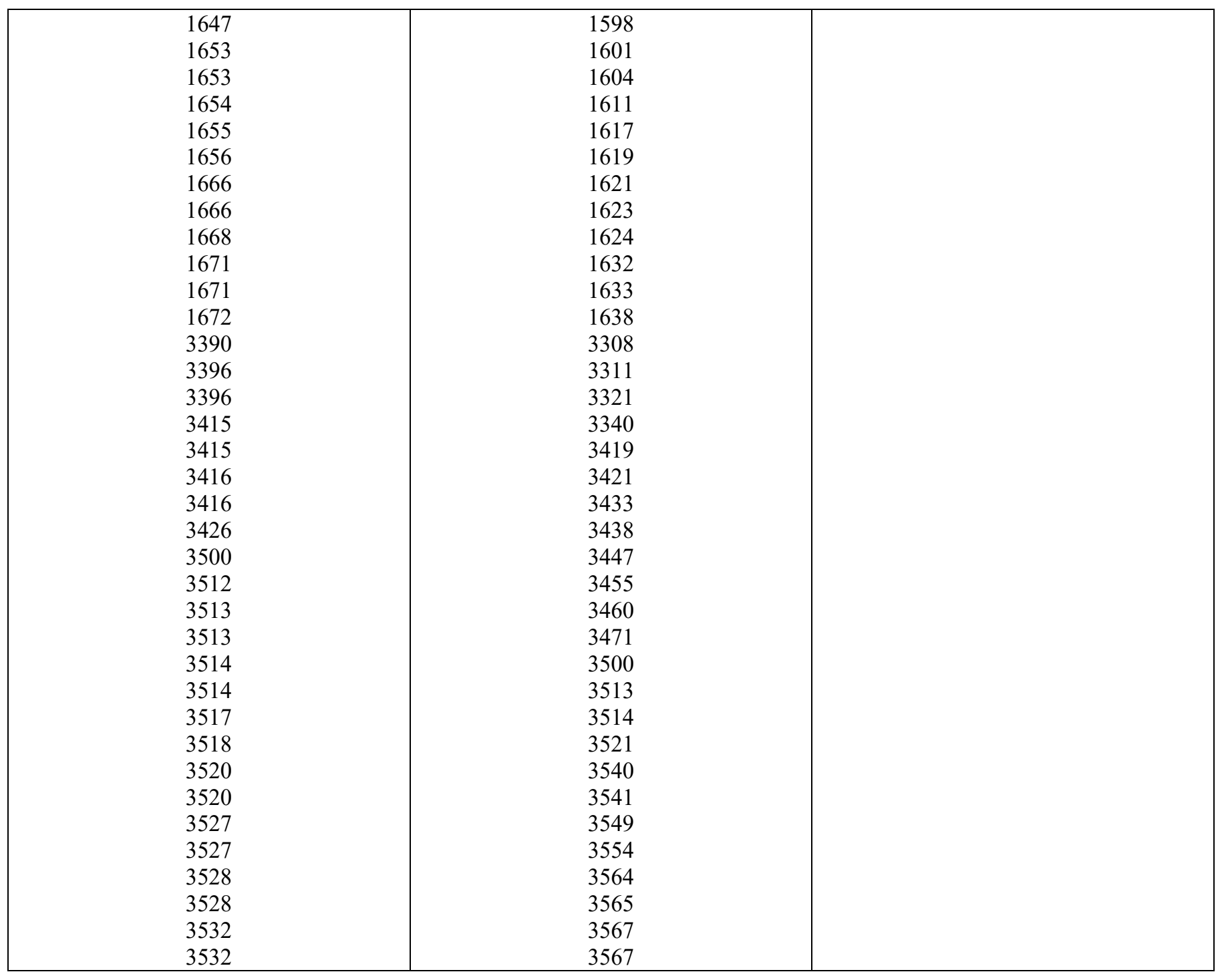


Table S18: Cartesian coordinates $(\AA)$ for the $\mathrm{Sc}\left(\mathrm{NH}_{3}\right)_{n=6,8}{ }^{+}$triplet spin species optimized at CAM$\mathrm{B} 3 \mathrm{LYP} / \mathrm{cc}-\mathrm{pVTZ}(\mathrm{Sc}, \mathrm{N})$ aug-cc-pVTZ $(\mathrm{H})$ constrained in $\mathrm{C}_{2 h}(n=6)$ and $\mathrm{C}_{2 v}(n=8)$ symmetries used for the excitation energy calculations.

\begin{tabular}{|c|c|c|c|c|c|c|c|}
\hline \multicolumn{4}{|c|}{$\mathrm{Sc}\left(\mathrm{NH}_{3}\right)_{6}{ }^{+}$} & \multicolumn{4}{|c|}{$\mathrm{Sc}\left(\mathrm{NH}_{3}\right)_{8}{ }^{+}$} \\
\hline $\mathrm{Sc}$ & 0.000000 & 0.000000 & 0.000000 & $\mathrm{Sc}$ & 0.000000 & 0.000000 & -0.000084 \\
\hline $\mathrm{N}$ & -1.954507 & 1.396789 & 0.000000 & $\mathrm{~N}$ & 1.496486 & 1.496486 & 1.387946 \\
\hline $\mathrm{N}$ & 1.954507 & -1.396789 & 0.000000 & $\mathrm{~N}$ & -1.496486 & 1.496486 & 1.387946 \\
\hline $\mathrm{N}$ & 0.827131 & 1.418043 & 1.753640 & $\mathrm{~N}$ & -1.496486 & -1.496486 & 1.387946 \\
\hline $\mathrm{N}$ & 0.827131 & 1.418043 & -1.753640 & $\mathrm{~N}$ & 0.000000 & 2.115917 & -1.387890 \\
\hline $\mathrm{N}$ & -0.827131 & -1.418043 & 1.753640 & $\mathrm{~N}$ & 0.000000 & -2.115917 & -1.387890 \\
\hline $\mathrm{N}$ & -0.827131 & -1.418043 & -1.753640 & $\mathrm{~N}$ & 2.115917 & 0.000000 & -1.387890 \\
\hline $\mathrm{H}$ & 1.126668 & 2.326150 & 1.399313 & $\mathrm{~N}$ & 1.496486 & -1.496486 & 1.387946 \\
\hline $\mathrm{H}$ & 1.126668 & 2.326150 & -1.399313 & $\mathrm{~N}$ & -2.115917 & 0.000000 & -1.387890 \\
\hline $\mathrm{H}$ & -1.126668 & -2.326150 & 1.399313 & $\mathrm{H}$ & 1.346886 & 2.492636 & 1.246616 \\
\hline $\mathrm{H}$ & -1.126668 & -2.326150 & -1.399313 & $\mathrm{H}$ & 1.331698 & 1.331698 & 2.379280 \\
\hline $\mathrm{H}$ & 0.160020 & 1.616896 & 2.498075 & $\mathrm{H}$ & 2.492636 & 1.346886 & 1.246616 \\
\hline $\mathrm{H}$ & 0.160020 & 1.616896 & -2.498075 & $\mathrm{H}$ & -2.492636 & 1.346886 & 1.246616 \\
\hline $\mathrm{H}$ & -0.160020 & -1.616896 & 2.498075 & $\mathrm{H}$ & -1.331698 & 1.331698 & 2.379280 \\
\hline $\mathrm{H}$ & -0.160020 & -1.616896 & -2.498075 & $\mathrm{H}$ & -1.346886 & 2.492636 & 1.246616 \\
\hline $\mathrm{H}$ & 1.645840 & 1.020101 & 2.213377 & $\mathrm{H}$ & -1.346886 & -2.492636 & 1.246616 \\
\hline $\mathrm{H}$ & 1.645840 & 1.020101 & -2.213377 & $\mathrm{H}$ & -1.331698 & -1.331698 & 2.379280 \\
\hline $\mathrm{H}$ & -1.645840 & -1.020101 & 2.213377 & $\mathrm{H}$ & -2.492636 & -1.346886 & 1.246616 \\
\hline $\mathrm{H}$ & -1.645840 & -1.020101 & -2.213377 & $\mathrm{H}$ & 2.492636 & -1.346886 & 1.246616 \\
\hline $\mathrm{H}$ & -1.769807 & 2.399104 & 0.000000 & $\mathrm{H}$ & 1.331698 & -1.331698 & 2.379280 \\
\hline $\mathrm{H}$ & 1.769807 & -2.399104 & 0.000000 & $\mathrm{H}$ & 1.346886 & -2.492636 & 1.246616 \\
\hline $\mathrm{H}$ & -2.546666 & 1.223714 & -0.812021 & $\mathrm{H}$ & 0.810162 & 2.714536 & -1.246613 \\
\hline $\mathrm{H}$ & -2.546666 & 1.223714 & 0.812021 & $\mathrm{H}$ & 0.000000 & 1.882824 & -2.379232 \\
\hline $\mathrm{H}$ & 2.546666 & -1.223714 & -0.812021 & $\mathrm{H}$ & -0.810162 & 2.714536 & -1.246613 \\
\hline $\mathrm{H}$ & 2.546666 & -1.223714 & 0.812021 & $\mathrm{H}$ & -0.810162 & -2.714536 & -1.246613 \\
\hline & & & & $\mathrm{H}$ & 0.000000 & -1.882824 & -2.379232 \\
\hline & & & & $\mathrm{H}$ & 0.810162 & -2.714536 & -1.246613 \\
\hline & & & & $\mathrm{H}$ & 2.714536 & -0.810162 & -1.246613 \\
\hline & & & & $\mathrm{H}$ & 1.882824 & 0.000000 & -2.379232 \\
\hline & & & & $\mathrm{H}$ & 2.714536 & 0.810162 & -1.246613 \\
\hline & & & & $\mathrm{H}$ & -2.714536 & 0.810162 & -1.246613 \\
\hline & & & & $\mathrm{H}$ & -1.882824 & 0.000000 & -2.379232 \\
\hline & & & & $\mathrm{H}$ & -2.714536 & -0.810162 & -1.246613 \\
\hline
\end{tabular}


Table S19: Electronic configurations and vertical excitation energies $\Delta \mathrm{E}(\mathrm{eV})$ for the low-lying electronic states of $\mathrm{Sc}\left(\mathrm{NH}_{3}\right)_{6}{ }^{+}$at $\mathrm{CASSCF}$ and CASPT2 using the CAM-B3LYP optimized structure of the lowest triplet state and the cc-pVTZ $(\mathrm{Sc}, \mathrm{N})$ d-aug-cc-pVTZ $(\mathrm{H})$ basis set. The different colors signify states with the same electronic configurations.

\begin{tabular}{|c|c|c|c|c|c|}
\hline State $^{a}$ & $\begin{array}{c}\text { Electronic } \\
\text { Configuration }\end{array}$ & $\begin{array}{l}\text { Irrep } \\
\left(\mathrm{C}_{2 \mathrm{~h}}\right)^{b}\end{array}$ & $\begin{array}{c}\Delta \mathrm{E} \\
(\mathrm{CASSCF})\end{array}$ & $\begin{array}{c}\Delta \mathrm{E} \\
(\mathrm{CASPT} 2)\end{array}$ & $\Delta \Delta \mathrm{E}^{c}$ \\
\hline \multirow{3}{*}{${ }^{3} \mathrm{~T}_{2 \mathrm{~g}}$} & $\mathrm{t}_{2 \mathrm{~g}}{ }^{1}\left({ }^{2} \mathrm{~T}_{2 \mathrm{~g}}\right) 1 \mathrm{~s}^{1}\left({ }^{2} \mathrm{~A}_{1 \mathrm{~g}}\right)$ & ${ }^{3} \mathrm{Ag}_{\mathrm{g}}$ & 0.000 & 0.000 & 0.000 \\
\hline & & ${ }^{3} \mathrm{Ag}_{\mathrm{g}}$ & 0.131 & 0.128 & -0.003 \\
\hline & & ${ }^{3} \mathrm{Bg}_{\mathrm{g}}$ & 0.132 & 0.137 & 0.005 \\
\hline \multirow[t]{3}{*}{${ }^{1} \mathrm{~T}_{2 \mathrm{~g}}$} & $\mathrm{t}_{2 \mathrm{~g}}{ }^{1}\left({ }^{2} \mathrm{~T}_{2 \mathrm{~g}}\right) 1 \mathrm{~s}^{1}\left({ }^{2} \mathrm{~A}_{1 \mathrm{~g}}\right)$ & ${ }^{1} \mathrm{Ag}_{\mathrm{g}}$ & 0.011 & 0.004 & -0.007 \\
\hline & & ${ }^{1} \mathrm{Ag}$ & 0.148 & 0.145 & -0.003 \\
\hline & & ${ }^{1} \mathrm{Bg}_{\mathrm{g}}$ & 0.149 & 0.154 & 0.005 \\
\hline \multirow[t]{9}{*}{${ }^{3} \mathrm{~A}_{2 \mathrm{u}}+{ }^{3} \mathrm{E}_{\mathrm{u}}+{ }^{3} \mathrm{~T} 1 \mathrm{u}+{ }^{3} \mathrm{~T} 2 \mathrm{u}$} & $\mathrm{t}_{2 \mathrm{~g}}{ }^{1}\left({ }^{2} \mathrm{~T}_{2 \mathrm{~g}}\right) 1 \mathrm{p}^{1}\left({ }^{2} \mathrm{~T}_{1 \mathrm{u}}\right)$ & ${ }^{3} \mathrm{Au}_{\mathrm{u}}$ & 0.749 & 0.899 & 0.149 \\
\hline & & ${ }^{3} \mathrm{Bu}$ & 0.750 & 0.899 & 0.150 \\
\hline & & ${ }^{3} \mathrm{Bu}_{\mathrm{u}}$ & 0.770 & 0.905 & 0.136 \\
\hline & & ${ }^{3} \mathrm{~A}_{u}$ & 0.859 & 1.026 & 0.167 \\
\hline & & ${ }^{3} \mathrm{~B}_{\mathrm{u}}$ & 0.860 & 1.031 & 0.171 \\
\hline & & ${ }^{3} \mathrm{~A}_{u}$ & 0.892 & 1.057 & 0.165 \\
\hline & & ${ }^{3} \mathrm{~A}_{u}$ & 0.930 & 1.062 & 0.132 \\
\hline & & ${ }^{3} \mathrm{~B}_{\mathrm{u}}$ & 0.931 & 1.071 & 0.140 \\
\hline & & ${ }^{3} \mathrm{~B}_{\mathrm{u}}$ & 0.934 & 1.071 & 0.138 \\
\hline \multirow[t]{9}{*}{${ }^{1} \mathrm{~A}_{2 \mathrm{u}}+{ }^{1} \mathrm{E}_{\mathrm{u}}+{ }^{1} \mathrm{~T}_{1 \mathrm{u}}+{ }^{1} \mathrm{~T}_{2 \mathrm{u}}$} & $\mathrm{t}_{2 \mathrm{~g}}{ }^{1}\left({ }^{2} \mathrm{~T}_{2 \mathrm{~g}}\right) 1 \mathrm{p}^{1}\left({ }^{2} \mathrm{~T}_{1 \mathrm{u}}\right)$ & ${ }^{1} \mathrm{~A}_{\mathrm{u}}$ & 0.752 & 0.908 & 0.156 \\
\hline & & ${ }^{1} \mathrm{~B}_{\mathrm{u}}$ & 0.752 & 0.914 & 0.163 \\
\hline & & ${ }^{1} \mathrm{~B}_{\mathrm{u}}$ & 0.808 & 0.944 & 0.136 \\
\hline & & ${ }^{1} \mathrm{~A}_{\mathrm{u}}$ & 0.864 & 1.040 & 0.175 \\
\hline & & ${ }^{1} \mathrm{~B}_{\mathrm{u}}$ & 0.865 & 1.044 & 0.178 \\
\hline & & ${ }^{1} \mathrm{~A}_{\mathrm{u}}$ & 0.900 & 1.069 & 0.169 \\
\hline & & ${ }^{1} \mathrm{~A}_{\mathrm{u}}$ & 0.943 & 1.078 & 0.135 \\
\hline & & ${ }^{1} \mathrm{~B}_{\mathrm{u}}$ & 0.944 & 1.087 & 0.143 \\
\hline & & ${ }^{1} \mathrm{~B}_{\mathrm{u}}$ & 0.949 & 1.087 & 0.138 \\
\hline \multirow{12}{*}{$\begin{array}{c}{ }^{3} \mathrm{~A}_{1 \mathrm{~g}}+{ }^{3} \mathrm{E}_{\mathrm{g}}+{ }^{3} \mathrm{~T}_{1 \mathrm{~g}}+{ }^{3} \mathrm{~T}_{2 \mathrm{~g}} \\
{ }^{3} \mathrm{~T}_{1 \mathrm{~g}}+{ }^{3} \mathrm{~T}_{2 \mathrm{~g}}\end{array}$} & $\mathrm{t}_{2 \mathrm{~g}}{ }^{1}\left({ }^{2} \mathrm{~T}_{2 \mathrm{~g}}\right) 1 \mathrm{~d}^{1}\left({ }^{2} \mathrm{~T}_{2 \mathrm{~g}}+{ }^{2} \mathrm{E}_{\mathrm{g}}\right)$ & ${ }^{3} \mathrm{Ag}_{\mathrm{g}}$ & 1.530 & 1.826 & 0.296 \\
\hline & & ${ }^{3} \mathrm{~B}_{\mathrm{g}}$ & 1.531 & 1.833 & 0.303 \\
\hline & & ${ }^{3} \mathrm{Bg}_{\mathrm{g}}$ & 1.629 & 1.895 & 0.265 \\
\hline & & ${ }^{3} \mathrm{Ag}$ & 1.630 & 1.903 & 0.273 \\
\hline & & ${ }^{3} \mathrm{~B}_{\mathrm{g}}$ & 1.643 & 1.957 & 0.314 \\
\hline & & ${ }^{3} \mathrm{Ag}$ & 1.680 & 1.976 & 0.296 \\
\hline & & ${ }^{3} \mathrm{Bg}_{\mathrm{g}}$ & 1.680 & 1.978 & 0.298 \\
\hline & & ${ }^{3} \mathrm{Ag}$ & 1.710 & 1.987 & 0.277 \\
\hline & & ${ }^{3} \mathrm{Ag}$ & 1.773 & $2.061^{d}$ & $0.288^{e}$ \\
\hline & & ${ }^{3} \mathrm{Ag}_{\mathrm{g}}$ & 1.788 & 2.069 & 0.281 \\
\hline & & ${ }^{3} \mathrm{~B}_{\mathrm{g}}$ & 1.788 & $2.077^{d}$ & $0.288^{e}$ \\
\hline & & ${ }^{3} \mathrm{~B}_{\mathrm{g}}$ & 1.798 & 2.076 & 0.279 \\
\hline
\end{tabular}




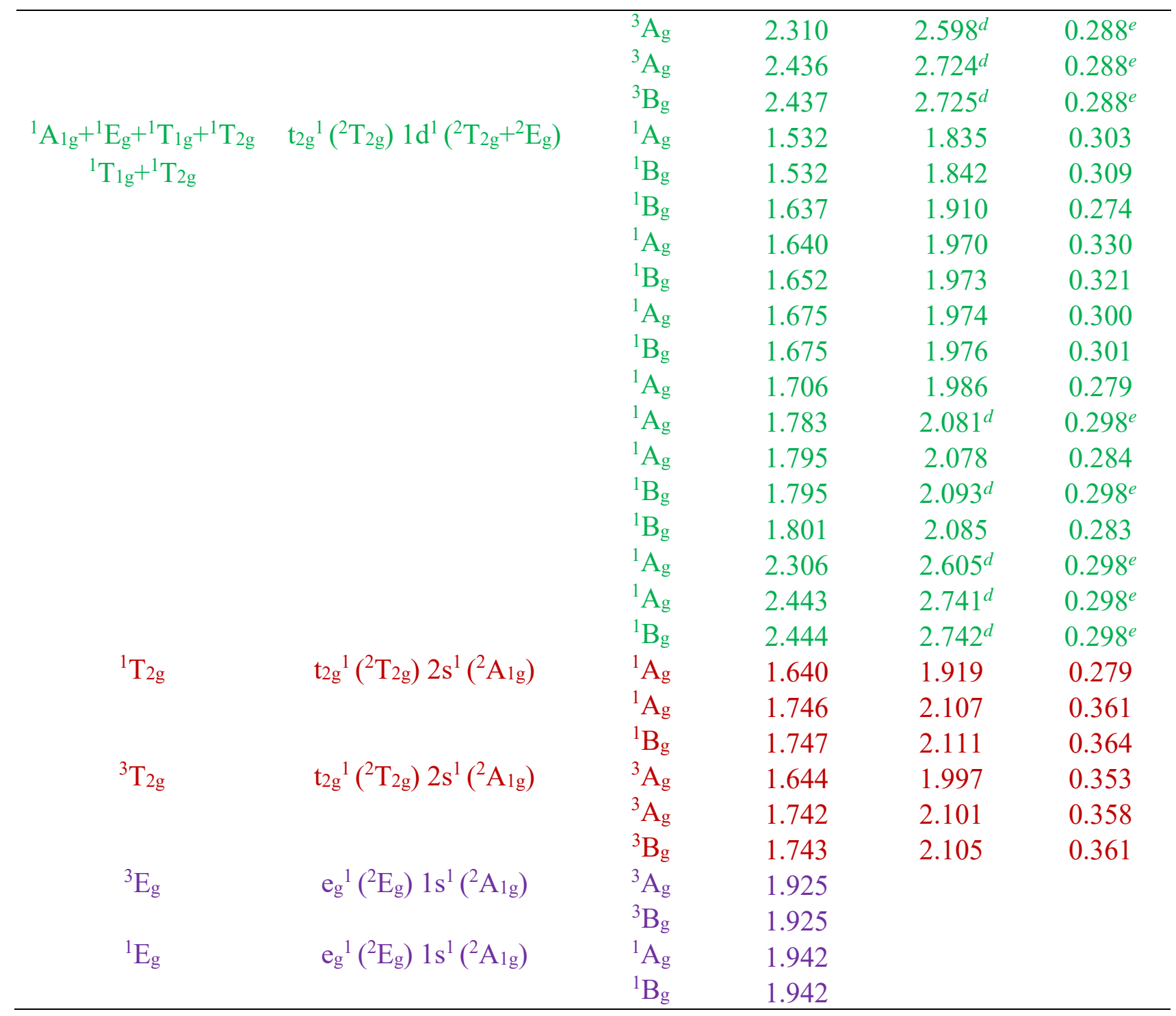

${ }^{a}$ Electronic terms under $\mathrm{O}_{\mathrm{h}}$ obtained based on the electronic configuration.

${ }^{b}$ Irreducible representation under $\mathrm{C}_{2} \mathrm{~h}$.

${ }^{c} \Delta \Delta \mathrm{E}=\Delta \mathrm{E}(\mathrm{CASPT} 2)-\Delta \mathrm{E}(\mathrm{CASSCF})$.

${ }^{d}$ Estimated values obtained as $\Delta \mathrm{E}(\mathrm{CASPT} 2)=\Delta \mathrm{E}(\mathrm{CASSCF})+\delta \mathrm{E}$ (see footnote e $)$.

${ }^{e} \delta \mathrm{E}$ value obtained as average $\Delta \Delta \mathrm{E}$ for states with identical electronic configuration. 
Table S20: Electronic configurations and vertical excitation energies $\triangle \mathrm{E}(\mathrm{eV})$ for the low-lying electronic states of $\mathrm{Sc}\left(\mathrm{NH}_{3}\right) 8_{8}^{+}$at $\mathrm{CASSCF}, \mathrm{CASPT} 2$, and $\mathrm{P} 3+$ using the CAM-B3LYP optimized structure of the lowest triplet state and the cc-pVDZ $(\mathrm{Sc}, \mathrm{N})$ d-aug-ccpVDZ $(\mathrm{H})$ or cc-pVTZ(Sc,N) d-aug-ccpVTZ $(\mathrm{H})$ basis sets (see footnotes). The different colors signify states with the same electronic configurations.

\begin{tabular}{|c|c|c|c|c|c|c|c|c|}
\hline State $^{a}$ & $\begin{array}{c}\text { Electronic } \\
\text { Configuration }^{b}\end{array}$ & $\begin{array}{l}\text { Irrep } \\
\left(\mathrm{C}_{2 \mathrm{v}}\right)^{c}\end{array}$ & $\begin{array}{c}\Delta \mathrm{E}^{d} \\
(\mathrm{CASSCF})\end{array}$ & $\begin{array}{c}\Delta \mathrm{E}^{d} \\
(\mathrm{CASPT} 2)\end{array}$ & $\Delta \Delta \mathrm{E}^{e}$ & $\begin{array}{c}\Delta \mathrm{E}^{d} \\
(\mathrm{P} 3+/ \mathrm{DZ})\end{array}$ & $\begin{array}{c}\Delta \mathrm{E}^{f} \\
(\mathrm{P} 3+/ \mathrm{TZ})\end{array}$ & $\Delta \mathrm{DT}^{g}$ \\
\hline${ }^{3} \mathrm{~A}_{1}$ & $3 d_{a_{1}}^{1} 1 s_{a_{1}}^{1}$ & ${ }^{3} \mathrm{~A}_{1}$ & 0.000 & 0.000 & 0.000 & 0.000 & 0.000 & 0.000 \\
\hline${ }^{1} \mathrm{~A}_{1}$ & $3 d_{a_{1}}^{1} 1 s_{a_{1}}^{1}$ & ${ }^{1} \mathrm{~A}_{1}$ & 0.009 & 0.003 & -0.006 & 0.002 & 0.003 & 0.001 \\
\hline${ }^{3} \mathrm{~B}_{2}$ & $3 d_{a_{1}}^{1} 1 p_{b_{2}}^{1}$ & ${ }^{3} \mathrm{~A}_{1}$ & 0.618 & 0.741 & 0.122 & 0.761 & 0.768 & 0.007 \\
\hline${ }^{1} \mathrm{~B}_{2}$ & $3 d_{a_{1}}^{1} 1 p_{b_{2}}^{1}$ & ${ }^{1} \mathrm{~A}_{1}$ & 0.636 & 0.759 & 0.123 & 0.775 & 0.781 & 0.006 \\
\hline \multirow[t]{2}{*}{${ }^{3} \mathrm{E}_{1}$} & $3 d_{a_{1}}^{1} 1 p_{e_{1}}^{1}$ & ${ }^{3} \mathrm{~B}_{1}$ & 0.698 & 0.879 & 0.182 & 0.903 & 0.913 & 0.010 \\
\hline & $3 d_{a_{1}}^{1} 1 p_{e_{1}}^{1}$ & ${ }^{3} \mathrm{~B}_{2}$ & 0.698 & 0.879 & 0.182 & 0.903 & 0.913 & 0.010 \\
\hline \multirow[t]{2}{*}{${ }^{3} E_{2}$} & $3 d_{e_{2}}^{1} 1 s_{a_{1}}^{1}$ & ${ }^{3} \mathrm{~A}_{1}$ & 0.849 & 0.882 & 0.032 & & & \\
\hline & $3 d_{e_{2}}^{1} 1 s_{a_{1}}^{1}$ & ${ }^{3} \mathrm{~A}_{2}$ & 0.849 & 0.885 & 0.036 & & & \\
\hline \multirow[t]{2}{*}{${ }^{1} \mathrm{E}_{1}$} & $3 d_{a_{1}}^{1} 1 p_{e_{1}}^{1}$ & ${ }^{1} \mathrm{~B}_{1}$ & 0.701 & 0.886 & 0.185 & 0.904 & 0.914 & 0.010 \\
\hline & $3 d_{a_{1}}^{1} 1 p_{e_{1}}^{1}$ & ${ }^{1} \mathrm{~B}_{2}$ & 0.701 & 0.886 & 0.185 & 0.904 & 0.914 & 0.010 \\
\hline \multirow[t]{2}{*}{${ }^{1} \mathrm{E}_{2}$} & $3 d_{e_{2}}^{1} 1 s_{a_{1}}^{1}$ & ${ }^{1} \mathrm{~A}_{1}$ & 0.857 & 0.893 & 0.035 & & & \\
\hline & $3 d_{e_{2}}^{1} 1 s_{a_{1}}^{1}$ & ${ }^{1} \mathrm{~A}_{2}$ & 0.857 & 0.896 & 0.039 & & & \\
\hline \multirow[t]{2}{*}{${ }^{3} \mathrm{E}_{3}$} & $3 d_{e_{3}}^{1} 1 s_{a_{1}}^{1}$ & ${ }^{3} \mathrm{~B}_{1}$ & 1.161 & 1.214 & 0.053 & & & \\
\hline & $3 d_{e_{3}}^{1} 1 s_{a_{1}}^{1}$ & ${ }^{3} \mathrm{~B}_{2}$ & 1.161 & 1.214 & 0.053 & & & \\
\hline \multirow[t]{2}{*}{${ }^{1} \mathrm{E}_{3}$} & $3 d_{e_{3}}^{1} 1 s_{a_{1}}^{1}$ & ${ }^{1} \mathrm{~B}_{1}$ & 1.172 & 1.229 & 0.057 & & & \\
\hline & $3 d_{e_{3}}^{1} 1 s_{a_{1}}^{1}$ & ${ }^{1} \mathrm{~B}_{2}$ & 1.172 & 1.229 & 0.057 & & & \\
\hline${ }^{1} \mathrm{~A}_{1}$ & $3 d_{a_{1}}^{1} 1 d_{a_{1}}^{1}$ & ${ }^{1} \mathrm{~A}_{1}$ & 1.269 & 1.552 & 0.283 & 1.586 & 1.601 & 0.015 \\
\hline${ }^{3} \mathrm{~A}_{1}$ & $3 d_{a_{1}}^{1} 1 d_{a_{1}}^{1}$ & ${ }^{3} \mathrm{~A}_{1}$ & 1.279 & 1.560 & 0.281 & 1.591 & 1.606 & 0.015 \\
\hline \multirow[t]{2}{*}{${ }^{3} \mathrm{E}_{2}$} & $3 d_{e_{2}}^{1} 1 p_{b_{2}}^{1}$ & ${ }^{3} \mathrm{~A}_{1}$ & 1.431 & 1.623 & 0.191 & & & \\
\hline & $3 d_{e_{2}}^{1} 1 p_{b_{2}}^{1}$ & ${ }^{3} \mathrm{~A}_{2}$ & 1.431 & 1.623 & 0.191 & & & \\
\hline \multirow[t]{2}{*}{${ }^{1} \mathrm{E}_{2}$} & $3 d_{e_{2}}^{1} 1 p_{b_{2}}^{1}$ & ${ }^{1} \mathrm{~A}_{1}$ & 1.434 & 1.631 & 0.196 & & & \\
\hline & $3 d_{e_{2}}^{1} 1 p_{b_{2}}^{1}$ & ${ }^{1} \mathrm{~A}_{2}$ & 1.434 & 1.631 & 0.196 & & & \\
\hline \multirow[t]{2}{*}{${ }^{3} \mathrm{E}_{2}$} & $3 d_{a_{1}}^{1} 1 d_{e_{2}}^{1}$ & ${ }^{3} \mathrm{~A}_{2}$ & 1.384 & 1.687 & 0.302 & 1.731 & 1.747 & 0.016 \\
\hline & $3 d_{a_{1}}^{1} 1 d_{e_{2}}^{1}$ & ${ }^{3} \mathrm{~A}_{1}$ & 1.384 & 1.696 & 0.312 & 1.731 & 1.747 & 0.016 \\
\hline \multirow[t]{2}{*}{${ }^{1} \mathrm{E}_{2}$} & $3 d_{a_{1}}^{1} 1 d_{e_{2}}^{1}$ & ${ }^{1} \mathrm{~A}_{2}$ & 1.386 & 1.690 & 0.304 & 1.731 & 1.747 & 0.016 \\
\hline & $3 d_{a_{1}}^{1} 1 d_{e_{2}}^{1}$ & ${ }^{1} \mathrm{~A}_{1}$ & 1.386 & 1.700 & 0.314 & 1.731 & 1.747 & 0.016 \\
\hline \multirow[t]{2}{*}{${ }^{3} E_{3}$} & $3 d_{a_{1}}^{1} 1 d_{e_{3}}^{1}$ & ${ }^{3} \mathrm{~B}_{1}$ & 1.476 & 1.784 & 0.308 & 1.824 & 1.842 & 0.018 \\
\hline & $3 d_{a_{1}}^{1} 1 d_{e_{3}}^{1}$ & ${ }^{3} \mathrm{~B}_{2}$ & 1.476 & 1.784 & 0.308 & 1.824 & 1.842 & 0.018 \\
\hline \multirow[t]{4}{*}{${ }^{3}\left(\mathrm{E}_{1}+\mathrm{E}_{3}\right)$} & $3 d_{e_{2}}^{1} 1 p_{e_{1}}^{1}$ & ${ }^{3} \mathrm{~B}_{1}$ & 1.591 & 1.787 & 0.196 & & & \\
\hline & $3 d_{e_{2}}^{1} 1 p_{e_{1}}^{1}$ & ${ }^{3} \mathrm{~B}_{2}$ & 1.591 & 1.787 & 0.196 & & & \\
\hline & $3 d_{e_{2}}^{1} 1 p_{e_{1}}^{1}$ & ${ }^{3} \mathrm{~B}_{1}$ & 1.592 & 1.788 & 0.196 & & & \\
\hline & $3 d_{e_{2}}^{1} 1 p_{e_{1}}^{1}$ & ${ }^{3} \mathrm{~B}_{2}$ & 1.592 & 1.788 & 0.196 & & & \\
\hline \multirow[t]{2}{*}{${ }^{1} \mathrm{E}_{3}$} & $3 d_{a_{1}}^{1} 1 d_{e_{3}}^{1}$ & ${ }^{1} \mathrm{~B}_{1}$ & 1.477 & 1.790 & 0.312 & & & \\
\hline & $3 d_{a_{1}}^{1} 1 d_{e_{3}}^{1}$ & ${ }^{1} \mathrm{~B}_{2}$ & 1.477 & 1.790 & 0.312 & & & \\
\hline
\end{tabular}




\begin{tabular}{clllll}
${ }^{1}\left(\mathrm{E}_{1}+\mathrm{E}_{3}\right)$ & $3 d_{e_{2}}^{1} 1 p_{e_{1}}^{1}$ & ${ }^{1} \mathrm{~B}_{1}$ & 1.594 & 1.793 & 0.199 \\
& $3 d_{e_{2}}^{1} 1 p_{e_{1}}^{1}$ & ${ }^{1} \mathrm{~B}_{2}$ & 1.594 & 1.793 & 0.199 \\
& $3 d_{e_{2}}^{1} 1 p_{e_{1}}^{1}$ & ${ }^{1} \mathrm{~B}_{1}$ & 1.597 & 1.796 & 0.199 \\
${ }^{3}\left(\mathrm{E}_{1}+\mathrm{E}_{3}\right) ?^{h}$ & $3 d_{e_{2}}^{1} 1 p_{e_{1}}^{1}$ & ${ }^{1} \mathrm{~B}_{2}$ & 1.597 & 1.796 & 0.199 \\
& $3 d_{e_{2}}^{1} 3 d_{e_{3}}^{1}$ & ${ }^{3} \mathrm{~B}_{1}$ & 1.769 & 1.958 & 0.189 \\
${ }^{3}\left(\mathrm{E}_{1}+\mathrm{E}_{3}\right) ?^{h}$ & $3 d_{e_{2}}^{1} 3 d_{e_{3}}^{1}$ & ${ }^{3} \mathrm{~B}_{2}$ & 1.769 & 1.958 & 0.189 \\
& $3 d_{e_{2}}^{1} 3 d_{e_{3}}^{1}$ & ${ }^{1} \mathrm{~B}_{1}$ & 1.776 & 1.972 & 0.196 \\
${ }^{1}\left(\mathrm{~B}_{1}+\mathrm{B}_{2}+\mathrm{E}_{2}\right)$ & $3 d_{e_{2}}^{1} 3 d_{e_{3}}^{1}$ & ${ }^{1} \mathrm{~B}_{2}$ & 1.776 & 1.972 & 0.196 \\
& $3 d_{e_{3}}^{1} 1 p_{e_{1}}^{1}$ & ${ }^{1} \mathrm{~A}_{2}$ & 1.857 & 2.100 & 0.243 \\
& $3 d_{e_{3}}^{1} 1 p_{e_{1}}^{1}$ & ${ }^{1} \mathrm{~A}_{2}$ & 1.894 & 2.129 & 0.236 \\
& $3 d_{e_{3}}^{1} 1 p_{e_{1}}^{1}$ & ${ }^{1} \mathrm{~A}_{1}$ & 1.894 & 2.131 & 0.237 \\
${ }^{3}\left(\mathrm{~B}_{1}+\mathrm{B}_{2}+\mathrm{E}_{2}\right)$ & $3 d_{e_{3}}^{1} 1 p_{e_{1}}^{1}$ & ${ }^{1} \mathrm{~A}_{1}$ & 1.924 & 2.150 & 0.227 \\
& $3 d_{e_{3}}^{1} 1 p_{e_{1}}^{1}$ & ${ }^{3} \mathrm{~A}_{2}$ & 1.859 & 2.101 & 0.242 \\
& $3 d_{e_{3}}^{1} 1 p_{e_{1}}^{1}$ & ${ }^{3} \mathrm{~A}_{2}$ & 1.886 & 2.116 & 0.230 \\
& $3 d_{e_{3}}^{1} 1 p_{e_{1}}^{1}$ & ${ }^{3} \mathrm{~A}_{1}$ & 1.886 & 2.117 & 0.231 \\
& $3 d_{e_{3}}^{1} 1 p_{e_{1}}^{1}$ & ${ }^{3} \mathrm{~A}_{1}$ & 1.914 & 2.136 & 0.222 \\
\hline
\end{tabular}

${ }^{a}$ Electronic terms under $\mathrm{D}_{4 d}$ obtained based on the electronic configuration.

${ }^{b} 1 \mathrm{~s}, 1 \mathrm{p}, 1 \mathrm{~d}$ notation is for diffuse outer $\mathrm{s}, \mathrm{p}, \mathrm{d}$ orbital respectively.

${ }^{c}$ Irreducible representation under $\mathrm{C}_{2 v}$.

${ }^{d}$ The cc-pVDZ(Sc,N) d-aug-ccpVDZ(H) basis set is used.

${ }^{e} \Delta \Delta \mathrm{E}=\Delta \mathrm{E}(\mathrm{CASPT} 2)-\Delta \mathrm{E}(\mathrm{CASSCF})$.

$f$ The cc-pVTZ(Sc,N) d-aug-ccpVTZ(H) basis set is used.

${ }^{g} \Delta \mathrm{DT}=\Delta \mathrm{E}(\mathrm{P} 3+/ \mathrm{TZ})-\Delta \mathrm{E}(\mathrm{P} 3+/ \mathrm{DZ})$.

${ }^{h}$ One or more components of the same configuration are high in energy and beyond the tabulated energy range. 
Table S21: Electronic energies (a.u.) for the $\mathrm{Sc}\left(\mathrm{NH}_{3}\right)_{\mathrm{n}=1-8}$ species optimized at CAM-B3LYP with the cc-pVTZ $(\mathrm{Sc}, \mathrm{N}) /$ aug-cc-pVTZ $(\mathrm{H})$ basis set for the lowest doublet and quartet state.

\begin{tabular}{clccc}
\hline$n$ & \multicolumn{2}{c}{ CAM-B3LYP } & \multicolumn{2}{c}{ MP2//CAM-B3LYP } \\
\hline & Doublet & Quartet & Doublet & Quartet \\
\hline 0 & -760.655119 & -760.617281 & -759.768727 & -759.709860 \\
1 & -817.236613 & -817.201051 & -816.240926 & -816.193280 \\
2 & -873.824008 & -873.782838 & -872.719925 & -872.667779 \\
3 & -930.394774 & -930.366308 & -929.194093 & -929.179934 \\
4 & -986.968593 & -986.949668 & -985.675777 & -985.664441 \\
5 & -1043.542670 & -1043.529020 & -1042.162747 & -1042.146296 \\
6 & -1100.124530 & -1100.112990 & -1098.648793 & -1098.632738 \\
$6^{a}$ & -1100.124527 & & & \\
7 & -1156.687020 & -1156.654840 & -1155.116580 & -1155.078960 \\
8 & -1213.250140 & -1213.239080 & -1211.588868 & -1211.572535 \\
$8^{a}$ & -1213.250186 & & & \\
\hline
\end{tabular}

${ }^{a}$ Energies of the imposed $\mathrm{C}_{2 \mathrm{~h}}$ or $\mathrm{C}_{2 \mathrm{v}}$ structures for $n=6$ or 8 . 
Table S22: Cartesian coordinates $(\AA)$ for the $\mathrm{Sc}\left(\mathrm{NH}_{3}\right)_{\mathrm{n}=1-8}$ species (doublet and quartet spin multiplicities) optimized at CAM-B3LYP with the cc-pVTZ(Sc,N) aug-cc-pVTZ(H) basis set.

\begin{tabular}{|c|c|c|c|c|c|c|c|}
\hline \multicolumn{4}{|c|}{ Doublet } & \multicolumn{4}{|c|}{ Quartet } \\
\hline \multicolumn{8}{|c|}{$\mathrm{Sc}\left(\mathrm{NH}_{3}\right)_{1}$} \\
\hline $\mathrm{N}$ & 1.520566 & -0.000249 & -0.000135 & $\bar{N}$ & 1.591613 & -0.000113 & 0.000090 \\
\hline $\mathrm{H}$ & 1.877563 & 0.953759 & -0.000514 & $\mathrm{H}$ & 1.966719 & 0.619707 & -0.713238 \\
\hline $\mathrm{H}$ & 1.884366 & -0.473643 & 0.825376 & $\mathrm{H}$ & 1.969166 & 0.308203 & 0.892320 \\
\hline $\mathrm{H}$ & 1.887638 & -0.475586 & -0.823034 & $\mathrm{H}$ & 1.969632 & -0.926524 & -0.180226 \\
\hline $\mathrm{Sc}$ & -0.775882 & -0.000133 & -0.000042 & $\mathrm{Sc}$ & -0.811753 & -0.000028 & 0.000024 \\
\hline \multicolumn{8}{|c|}{$\mathrm{Sc}\left(\mathrm{NH}_{3}\right)_{2}$} \\
\hline $\mathrm{N}$ & 2.300937 & 0.000792 & 0.001291 & $\mathrm{~N}$ & 2.332985 & -0.005227 & 0.000773 \\
\hline $\mathrm{H}$ & 2.654927 & -0.939671 & 0.166301 & $\mathrm{H}$ & 2.671719 & 0.501690 & 0.812715 \\
\hline $\mathrm{H}$ & 2.661825 & 0.328086 & -0.893223 & $\mathrm{H}$ & 2.741064 & -0.932902 & 0.004106 \\
\hline $\mathrm{H}$ & 2.656820 & 0.612528 & 0.733708 & $\mathrm{H}$ & 2.672544 & 0.495937 & -0.814432 \\
\hline $\mathrm{N}$ & -2.300939 & 0.000697 & 0.001417 & $\mathrm{~N}$ & -2.332822 & 0.006958 & 0.000696 \\
\hline $\mathrm{H}$ & -2.659392 & -0.642368 & -0.702417 & $\mathrm{H}$ & -2.673149 & -0.492484 & 0.816642 \\
\hline $\mathrm{H}$ & -2.660037 & -0.286292 & 0.910343 & $\mathrm{H}$ & -2.738549 & 0.935689 & -0.003192 \\
\hline $\mathrm{H}$ & -2.654150 & 0.933367 & -0.204333 & $\mathrm{H}$ & -2.673414 & -0.499837 & -0.810574 \\
\hline $\mathrm{Sc}$ & 0.000001 & -0.000765 & -0.001397 & Sc & -0.000065 & -0.000962 & -0.000740 \\
\hline \multicolumn{8}{|c|}{$\mathrm{Sc}\left(\mathrm{NH}_{3}\right)_{3}$} \\
\hline $\mathrm{N}$ & 1.570010 & -1.482557 & -0.000010 & $\mathrm{~N}$ & 1.529728 & -1.599873 & -0.004098 \\
\hline $\mathrm{H}$ & 1.195039 & -2.426498 & -0.000041 & $\mathrm{H}$ & 0.995084 & -2.466413 & 0.014154 \\
\hline $\mathrm{H}$ & 2.161472 & -1.395382 & -0.827424 & $\mathrm{H}$ & 1.998021 & -1.595091 & -0.907467 \\
\hline $\mathrm{H}$ & 2.161413 & -1.395414 & 0.827449 & $\mathrm{H}$ & 2.236330 & -1.644583 & 0.725947 \\
\hline $\mathrm{N}$ & 1.529079 & 1.508792 & 0.000026 & $\mathrm{~N}$ & 1.460363 & 1.642871 & -0.027513 \\
\hline $\mathrm{H}$ & 2.121966 & 1.430721 & 0.827443 & $\mathrm{H}$ & 1.956288 & 1.633054 & 0.861763 \\
\hline $\mathrm{H}$ & 2.121980 & 1.430749 & -0.827384 & $\mathrm{H}$ & 2.142945 & 1.709143 & -0.778961 \\
\hline $\mathrm{H}$ & 1.138695 & 2.446386 & 0.000039 & $\mathrm{H}$ & 0.913443 & 2.501392 & -0.016036 \\
\hline $\mathrm{N}$ & -2.564994 & -0.005101 & 0.000008 & $\mathrm{~N}$ & -2.555004 & -0.012226 & -0.023903 \\
\hline $\mathrm{H}$ & -2.914146 & -0.487289 & 0.828817 & $\mathrm{H}$ & -2.904945 & -0.618933 & 0.712465 \\
\hline $\mathrm{H}$ & -2.935408 & 0.944526 & -0.000164 & $\mathrm{H}$ & -2.960731 & 0.905579 & 0.117688 \\
\hline $\mathrm{H}$ & -2.914187 & -0.487601 & -0.828602 & $\mathrm{H}$ & -2.876969 & -0.391027 & -0.907939 \\
\hline $\mathrm{Sc}$ & -0.279785 & -0.009911 & -0.000014 & $\mathrm{Sc}$ & -0.216432 & -0.011835 & 0.026999 \\
\hline \multicolumn{8}{|c|}{$\mathrm{Sc}\left(\mathrm{NH}_{3}\right)_{4}$} \\
\hline $\mathrm{Sc}$ & 0.000000 & 0.000002 & -0.000031 & $\mathrm{Sc}$ & 0.104837 & -0.458165 & -0.099879 \\
\hline $\mathrm{N}$ & 1.808177 & 1.521980 & -0.000074 & $\mathrm{~N}$ & 0.445958 & 1.322900 & 1.460011 \\
\hline $\mathrm{H}$ & 2.398220 & 1.414753 & -0.827054 & $\mathrm{H}$ & 0.932989 & 2.129076 & 1.064665 \\
\hline $\mathrm{H}$ & 2.397960 & 1.414938 & 0.827115 & $\mathrm{H}$ & 0.896270 & 1.119677 & 2.351991 \\
\hline $\mathrm{N}$ & -1.808178 & 1.521978 & 0.000074 & $\mathrm{~N}$ & -0.966525 & 1.234922 & -1.397509 \\
\hline $\mathrm{H}$ & -2.398233 & 1.414809 & -0.826904 & $\mathrm{H}$ & -1.450670 & 0.946855 & -2.247067 \\
\hline $\mathrm{H}$ & -1.471904 & 2.481670 & -0.000032 & $\mathrm{H}$ & -0.273378 & 1.930136 & -1.683898 \\
\hline $\mathrm{N}$ & -1.808173 & -1.521981 & -0.000228 & $\mathrm{~N}$ & -2.086178 & -1.091322 & 0.498462 \\
\hline $\mathrm{H}$ & -1.471899 & -2.481673 & -0.000291 & $\mathrm{H}$ & -2.040896 & -1.871699 & 1.145924 \\
\hline $\mathrm{H}$ & -2.398074 & -1.414789 & -0.827314 & $\mathrm{H}$ & -2.631164 & -1.406487 & -0.299849 \\
\hline $\mathrm{N}$ & 1.808174 & -1.521980 & 0.000268 & $\mathrm{~N}$ & 2.430039 & -0.632258 & -0.379834 \\
\hline $\mathrm{H}$ & 1.471895 & -2.481671 & 0.000189 & $\mathrm{H}$ & 2.522407 & -1.394370 & -1.045812 \\
\hline $\mathrm{H}$ & 2.398259 & -1.414847 & -0.826693 & $\mathrm{H}$ & 2.944850 & 0.170075 & -0.731039 \\
\hline $\mathrm{H}$ & -2.397949 & 1.414879 & 0.827265 & $\mathrm{H}$ & -1.643369 & 1.760791 & -0.837961 \\
\hline $\mathrm{H}$ & 1.471904 & 2.481672 & -0.000236 & $\mathrm{H}$ & -0.488676 & 1.681603 & 1.689962 \\
\hline $\mathrm{H}$ & 2.397916 & -1.414851 & 0.827476 & $\mathrm{H}$ & 2.877484 & -0.943351 & 0.479035 \\
\hline $\mathrm{H}$ & -2.398098 & -1.414904 & 0.826855 & $\mathrm{H}$ & -2.610470 & -0.340528 & 0.943602 \\
\hline \multicolumn{8}{|c|}{$\mathrm{Sc}\left(\mathrm{NH}_{3}\right)_{5}$} \\
\hline $\mathrm{N}$ & -1.235646 & -1.052387 & -1.559519 & $\mathrm{~N}$ & 0.278695 & -0.017602 & 2.052266 \\
\hline
\end{tabular}




\begin{tabular}{|c|c|c|c|c|c|c|c|}
\hline $\mathrm{H}$ & -0.939220 & -0.771659 & -2.493499 & $\mathrm{H}$ & -0.152565 & 0.733052 & 2.590100 \\
\hline $\mathrm{H}$ & -1.145848 & -2.070088 & -1.518204 & $\mathrm{H}$ & 0.042497 & -0.886292 & 2.531101 \\
\hline $\mathrm{H}$ & -2.235402 & -0.843408 & -1.493406 & $\mathrm{H}$ & 1.289497 & 0.098748 & 2.199119 \\
\hline $\mathrm{N}$ & -1.232856 & -1.051343 & 1.561590 & $\mathrm{~N}$ & 1.777963 & -1.539415 & -0.445501 \\
\hline $\mathrm{H}$ & -2.232709 & -0.842180 & 1.497279 & $\mathrm{H}$ & 2.508286 & -1.281598 & 0.222924 \\
\hline $\mathrm{H}$ & -1.143382 & -2.069102 & 1.520817 & $\mathrm{H}$ & 1.498357 & -2.485929 & -0.196174 \\
\hline $\mathrm{H}$ & -0.934568 & -0.770038 & 2.494814 & $\mathrm{H}$ & 2.220416 & -1.600109 & -1.359857 \\
\hline $\mathrm{N}$ & 1.887133 & 1.619031 & -0.000311 & $\mathrm{~N}$ & -1.879196 & 1.545297 & -0.213928 \\
\hline $\mathrm{H}$ & 1.595947 & 2.595766 & -0.005873 & $\mathrm{H}$ & -1.561821 & 2.508965 & -0.266889 \\
\hline $\mathrm{H}$ & 2.472107 & 1.485229 & 0.828426 & $\mathrm{H}$ & -2.378519 & 1.366300 & -1.084349 \\
\hline $\mathrm{H}$ & 2.480289 & 1.478423 & -0.822013 & $\mathrm{H}$ & -2.553457 & 1.493581 & 0.548420 \\
\hline $\mathrm{Sc}$ & 0.122449 & 0.097284 & -0.000506 & $\mathrm{Sc}$ & -0.048631 & 0.001267 & -0.329841 \\
\hline $\mathrm{N}$ & 1.814267 & -1.589828 & -0.000811 & $\mathrm{~N}$ & -1.823630 & -1.596504 & -0.289324 \\
\hline $\mathrm{H}$ & 1.395776 & -2.521353 & -0.002015 & $\mathrm{H}$ & -1.497934 & -2.555318 & -0.365660 \\
\hline $\mathrm{H}$ & 2.418793 & -1.546769 & -0.822566 & $\mathrm{H}$ & -2.514966 & -1.568499 & 0.458756 \\
\hline $\mathrm{H}$ & 2.417851 & -1.548399 & 0.821710 & $\mathrm{H}$ & -2.303380 & -1.393004 & -1.166596 \\
\hline $\mathrm{N}$ & -1.456732 & 1.904925 & -0.000209 & $\mathrm{~N}$ & 1.707520 & 1.602484 & -0.487145 \\
\hline $\mathrm{H}$ & -1.370487 & 2.501719 & -0.822596 & $\mathrm{H}$ & 1.395458 & 2.552725 & -0.301156 \\
\hline $\mathrm{H}$ & -2.418973 & 1.560152 & 0.004112 & $\mathrm{H}$ & 2.439663 & 1.410222 & 0.200744 \\
\hline $\mathrm{H}$ & -1.364774 & 2.505959 & 0.818461 & $\mathrm{H}$ & 2.160240 & 1.620728 & -1.398393 \\
\hline \multicolumn{8}{|c|}{$\mathrm{Sc}\left(\mathrm{NH}_{3}\right)_{6}$} \\
\hline $\mathrm{Sc}$ & 0.000000 & 0.000000 & 0.000000 & $\mathrm{Sc}$ & 0.000000 & 0.000000 & 0.000000 \\
\hline $\mathrm{N}$ & 1.720057 & 0.080774 & 1.657329 & $\mathrm{~N}$ & 1.313776 & -1.703450 & 1.069795 \\
\hline $\mathrm{H}$ & 2.099511 & 1.024024 & 1.755722 & $\mathrm{H}$ & 0.964777 & -2.025099 & 1.970146 \\
\hline $\mathrm{H}$ & 2.516612 & -0.531659 & 1.476217 & $\mathrm{H}$ & 2.237008 & -1.298646 & 1.253672 \\
\hline $\mathrm{N}$ & 0.206876 & -2.375926 & 0.154845 & $\mathrm{~N}$ & 1.816453 & 0.000904 & -1.560549 \\
\hline $\mathrm{H}$ & 0.230706 & -2.741272 & 1.107914 & $\mathrm{H}$ & 1.849184 & -0.813700 & -2.173607 \\
\hline $\mathrm{H}$ & 1.059067 & -2.706481 & -0.300959 & $\mathrm{H}$ & 2.709663 & -0.001073 & -1.055986 \\
\hline $\mathrm{N}$ & -1.720057 & -0.080774 & -1.657329 & $\mathrm{~N}$ & -1.313776 & 1.703450 & -1.069795 \\
\hline $\mathrm{H}$ & -1.361916 & 0.178366 & -2.578089 & $\mathrm{H}$ & -1.496698 & 2.537869 & -0.514630 \\
\hline $\mathrm{H}$ & -2.516612 & 0.531659 & -1.476217 & $\mathrm{H}$ & -2.237008 & 1.298646 & -1.253671 \\
\hline $\mathrm{N}$ & -0.206876 & 2.375926 & -0.154845 & $\mathrm{~N}$ & -1.816453 & -0.000904 & 1.560549 \\
\hline $\mathrm{H}$ & -1.059067 & 2.706481 & 0.300959 & $\mathrm{H}$ & -2.709663 & 0.001073 & 1.055986 \\
\hline $\mathrm{H}$ & 0.567471 & 2.848625 & 0.314576 & $\mathrm{H}$ & -1.851123 & -0.817651 & 2.170650 \\
\hline $\mathrm{H}$ & -0.567471 & -2.848625 & -0.314576 & $\mathrm{H}$ & 1.851123 & 0.817651 & -2.170650 \\
\hline $\mathrm{H}$ & 1.361916 & -0.178366 & 2.578089 & $\mathrm{H}$ & 1.496698 & -2.537869 & 0.514630 \\
\hline $\mathrm{H}$ & -0.230706 & 2.741272 & -1.107914 & $\mathrm{H}$ & -1.849184 & 0.813699 & 2.173607 \\
\hline $\mathrm{H}$ & -2.099511 & -1.024024 & -1.755722 & $\mathrm{H}$ & -0.964777 & 2.025099 & -1.970145 \\
\hline $\mathrm{N}$ & -1.771830 & -0.233103 & 1.585960 & $\mathrm{~N}$ & -1.317701 & -1.697243 & -1.074539 \\
\hline $\mathrm{H}$ & -2.445272 & -0.968983 & 1.368079 & $\mathrm{H}$ & -0.969635 & -2.016394 & -1.976139 \\
\hline $\mathrm{H}$ & -2.309330 & 0.630952 & 1.674492 & $\mathrm{H}$ & -2.240078 & -1.289654 & -1.256781 \\
\hline $\mathrm{H}$ & -1.412040 & -0.440325 & 2.519186 & $\mathrm{H}$ & -1.502456 & -2.533288 & -0.522418 \\
\hline $\mathrm{N}$ & 1.771830 & 0.233103 & -1.585960 & $\mathrm{~N}$ & 1.317701 & 1.697244 & 1.074539 \\
\hline $\mathrm{H}$ & 1.412040 & 0.440325 & -2.519186 & $\mathrm{H}$ & 1.502455 & 2.533288 & 0.522417 \\
\hline $\mathrm{H}$ & 2.309330 & -0.630952 & -1.674492 & $\mathrm{H}$ & 2.240078 & 1.289654 & 1.256781 \\
\hline $\mathrm{H}$ & 2.445272 & 0.968983 & -1.368079 & $\mathrm{H}$ & 0.969635 & 2.016394 & 1.976139 \\
\hline \multicolumn{8}{|c|}{$\mathrm{Sc}\left(\mathrm{NH}_{3}\right)_{7}$} \\
\hline $\mathrm{Sc}$ & -0.008478 & 0.000307 & 0.000412 & $\mathrm{Sc}$ & -0.049395 & 0.000249 & -0.000004 \\
\hline $\mathrm{N}$ & 0.632224 & 0.045576 & -2.340329 & $\mathrm{~N}$ & 1.493604 & -1.653132 & 0.775421 \\
\hline $\mathrm{H}$ & 1.169924 & -0.762934 & -2.654864 & $\mathrm{H}$ & 2.102177 & -1.372816 & 1.545966 \\
\hline $\mathrm{H}$ & 1.164972 & 0.868483 & -2.624480 & $\mathrm{H}$ & 2.096238 & -2.072476 & 0.065753 \\
\hline $\mathrm{N}$ & -0.941865 & 2.177938 & -0.710772 & $\mathrm{~N}$ & -0.613621 & -1.961227 & -1.344582 \\
\hline $\mathrm{H}$ & -1.728141 & 2.049856 & -1.349533 & $\mathrm{H}$ & -1.149763 & -2.642116 & -0.811852 \\
\hline $\mathrm{H}$ & -0.269405 & 2.758572 & -1.211189 & $\mathrm{H}$ & 0.204934 & -2.465498 & -1.698900 \\
\hline $\mathrm{N}$ & -0.005559 & -0.051155 & 2.459736 & $\mathrm{~N}$ & -0.614614 & 2.151257 & -1.013716 \\
\hline
\end{tabular}




\begin{tabular}{|c|c|c|c|c|c|c|c|}
\hline $\mathrm{H}$ & 0.925602 & -0.052622 & 2.873910 & $\mathrm{H}$ & 0.203398 & 2.706396 & -1.282993 \\
\hline $\mathrm{H}$ & -0.476222 & -0.879455 & 2.821471 & $\mathrm{H}$ & -1.151111 & 2.737526 & -0.378624 \\
\hline $\mathrm{N}$ & 1.851864 & -1.542536 & 0.373671 & $\mathrm{~N}$ & 1.491301 & 1.508984 & 1.032551 \\
\hline $\mathrm{H}$ & 1.487790 & -2.448321 & 0.665473 & $\mathrm{H}$ & 0.910244 & 2.196817 & 1.510554 \\
\hline $\mathrm{H}$ & 2.395842 & -1.708466 & -0.473365 & $\mathrm{H}$ & 2.100151 & 1.108455 & 1.747772 \\
\hline $\mathrm{H}$ & -1.291764 & 2.743941 & 0.062086 & $\mathrm{H}$ & -1.161641 & -1.710875 & -2.164248 \\
\hline $\mathrm{H}$ & -0.217841 & 0.053501 & -2.907941 & $\mathrm{H}$ & 0.914000 & -2.410372 & 1.135824 \\
\hline $\mathrm{H}$ & 2.521473 & -1.264872 & 1.090347 & $\mathrm{H}$ & 2.093315 & 2.038681 & 0.400456 \\
\hline $\mathrm{H}$ & -0.487787 & 0.755555 & 2.853568 & $\mathrm{H}$ & -1.162808 & 2.034947 & -1.862729 \\
\hline $\mathrm{N}$ & -2.413633 & -0.017834 & 0.580951 & $\mathrm{~N}$ & -2.529330 & -0.001728 & 0.013524 \\
\hline $\mathrm{H}$ & -3.020234 & -0.006082 & -0.239977 & $\mathrm{H}$ & -2.901384 & -0.854342 & 0.417488 \\
\hline $\mathrm{H}$ & -2.681763 & 0.788451 & 1.144264 & $\mathrm{H}$ & -2.906519 & 0.075864 & -0.924697 \\
\hline $\mathrm{H}$ & -2.675830 & -0.844708 & 1.116521 & $\mathrm{H}$ & -2.901110 & 0.773155 & 0.551933 \\
\hline $\mathrm{N}$ & 1.837017 & 1.545210 & 0.434756 & $\mathrm{~N}$ & 1.472274 & 0.148826 & -1.837668 \\
\hline $\mathrm{H}$ & 1.464155 & 2.436852 & 0.757375 & $\mathrm{H}$ & 0.881562 & 0.214540 & -2.665883 \\
\hline $\mathrm{H}$ & 2.382065 & 1.745859 & -0.404052 & $\mathrm{H}$ & 2.077241 & -0.660256 & -1.985908 \\
\hline $\mathrm{H}$ & 2.506931 & 1.248188 & 1.143359 & $\mathrm{H}$ & 2.076240 & 0.971877 & -1.855563 \\
\hline $\mathrm{N}$ & -0.925523 & -2.158018 & -0.785590 & $\mathrm{~N}$ & -0.593025 & -0.193411 & 2.374454 \\
\hline $\mathrm{H}$ & -1.270174 & -2.753691 & -0.032930 & $\mathrm{H}$ & -1.133292 & 0.603345 & 2.703286 \\
\hline $\mathrm{H}$ & -1.713600 & -2.013187 & -1.418531 & $\mathrm{H}$ & -1.131983 & -1.033388 & 2.571418 \\
\hline $\mathrm{H}$ & -0.249628 & -2.715634 & -1.307127 & $\mathrm{H}$ & 0.231276 & -0.241690 & 2.981150 \\
\hline \multicolumn{8}{|c|}{$\mathrm{Sc}\left(\mathrm{NH}_{3}\right)_{8}$} \\
\hline $\mathrm{H}$ & -0.240812 & -2.811523 & 1.234174 & $\mathrm{H}$ & -0.631341 & -2.744945 & 1.321078 \\
\hline $\mathrm{N}$ & -0.776871 & -1.957752 & 1.379496 & $\mathrm{~N}$ & -1.037939 & -1.817732 & 1.414204 \\
\hline $\mathrm{H}$ & -2.170957 & -1.809949 & -1.228137 & $\mathrm{H}$ & -2.397446 & -1.487158 & -1.315504 \\
\hline $\mathrm{H}$ & -1.752266 & -2.212434 & 1.233996 & $\mathrm{H}$ & -2.043016 & -1.939639 & 1.321028 \\
\hline $\mathrm{H}$ & -0.688229 & -1.733784 & 2.370488 & $\mathrm{H}$ & -0.880556 & -1.541656 & 2.384715 \\
\hline $\mathrm{H}$ & 0.310611 & -2.800578 & -1.242948 & $\mathrm{H}$ & -0.084568 & -2.811388 & -1.327982 \\
\hline $\mathrm{H}$ & 0.744862 & -1.708221 & -2.370299 & $\mathrm{H}$ & 0.473434 & -1.710179 & -2.384691 \\
\hline $\mathrm{N}$ & -1.935830 & -0.830551 & -1.379582 & $\mathrm{~N}$ & -2.020513 & -0.548055 & -1.414055 \\
\hline $\mathrm{N}$ & 0.830529 & -1.935532 & -1.379815 & $\mathrm{~N}$ & 0.548005 & -2.020135 & -1.414512 \\
\hline $\mathrm{H}$ & -2.811663 & 0.241061 & 1.234585 & $\mathrm{H}$ & -2.744986 & 0.631600 & 1.321390 \\
\hline $\mathrm{H}$ & -2.800953 & -0.311137 & -1.241260 & $\mathrm{H}$ & -2.811876 & 0.084234 & -1.326477 \\
\hline $\mathrm{H}$ & -1.709944 & -0.744276 & -2.370342 & $\mathrm{H}$ & -1.711533 & -0.473030 & -2.384509 \\
\hline $\mathrm{H}$ & 1.809733 & -2.171311 & -1.228149 & $\mathrm{H}$ & 1.486949 & -2.397466 & -1.315959 \\
\hline $\mathrm{Sc}$ & 0.000000 & 0.000000 & 0.000488 & $\mathrm{Sc}$ & 0.000001 & 0.000000 & 0.000162 \\
\hline $\mathrm{N}$ & -1.957734 & 0.776945 & 1.379631 & $\mathrm{~N}$ & -1.817678 & 1.038033 & 1.414286 \\
\hline $\mathrm{H}$ & 2.212354 & -1.752498 & 1.233765 & $\mathrm{H}$ & 1.939519 & -2.043261 & 1.320673 \\
\hline $\mathrm{H}$ & -1.733524 & 0.688306 & 2.370568 & $\mathrm{H}$ & -1.541442 & 0.880676 & 2.384754 \\
\hline $\mathrm{N}$ & 1.957863 & -0.777070 & 1.379379 & $\mathrm{~N}$ & 1.817790 & -1.038180 & 1.414037 \\
\hline $\mathrm{H}$ & -2.212242 & 1.752385 & 1.234126 & $\mathrm{H}$ & -1.939419 & 2.043123 & 1.321032 \\
\hline $\mathrm{H}$ & 1.733747 & -0.688516 & 2.370346 & $\mathrm{H}$ & 1.541631 & -0.880918 & 2.384542 \\
\hline $\mathrm{H}$ & 0.688425 & 1.733579 & 2.370579 & $\mathrm{H}$ & 0.880729 & 1.541423 & 2.384801 \\
\hline $\mathrm{H}$ & 2.811780 & -0.241176 & 1.234299 & $\mathrm{H}$ & 2.745093 & -0.631742 & 1.321108 \\
\hline $\mathrm{N}$ & 0.776998 & 1.957628 & 1.379599 & $\mathrm{~N}$ & 1.038051 & 1.817587 & 1.414305 \\
\hline $\mathrm{H}$ & 0.240940 & 2.811419 & 1.234387 & $\mathrm{H}$ & 0.631458 & 2.744812 & 1.321289 \\
\hline $\mathrm{N}$ & 1.935702 & 0.830676 & -1.379688 & $\mathrm{~N}$ & 2.020400 & 0.548202 & -1.414160 \\
\hline $\mathrm{H}$ & 1.709720 & 0.744495 & -2.370433 & $\mathrm{H}$ & 1.711342 & 0.473279 & -2.384596 \\
\hline $\mathrm{H}$ & 2.170846 & 1.810059 & -1.228173 & $\mathrm{H}$ & 2.397340 & 1.487295 & -1.315540 \\
\hline $\mathrm{H}$ & 1.752387 & 2.212308 & 1.234049 & $\mathrm{H}$ & 2.043124 & 1.939489 & 1.321073 \\
\hline $\mathrm{H}$ & 2.800836 & 0.311246 & -1.241498 & $\mathrm{H}$ & 2.811772 & -0.084095 & -1.326714 \\
\hline $\mathrm{N}$ & -0.830657 & 1.935655 & -1.379566 & $\mathrm{~N}$ & -0.548118 & 2.020281 & -1.414265 \\
\hline $\mathrm{H}$ & -1.809842 & 2.171433 & -1.227776 & $\mathrm{H}$ & -1.487049 & 2.397611 & -1.315592 \\
\hline $\mathrm{H}$ & -0.745097 & 1.708422 & -2.370078 & $\mathrm{H}$ & -0.473635 & 1.710416 & -2.384480 \\
\hline $\mathrm{H}$ & -0.310715 & 2.800685 & -1.242684 & $\mathrm{H}$ & 0.084470 & 2.811521 & -1.327718 \\
\hline
\end{tabular}


Table S23: Harmonic vibrational frequencies $\left(\mathrm{cm}^{-1}\right)$ for the $\mathrm{Sc}\left(\mathrm{NH}_{3}\right)_{\mathrm{n}=1-8}$ species (doublet and quartet spin multiplicities) optimized at CAM-B3LYP combined with the cc-pVTZ(Sc,N) aug-cc$\mathrm{pVTZ}(\mathrm{H})$ basis set.

\begin{tabular}{|c|c|}
\hline Doublet & Quartet \\
\hline \multicolumn{2}{|c|}{$\mathrm{Sc}\left(\mathrm{NH}_{3}\right)_{1}$} \\
\hline 322 & 257 \\
\hline 350 & 316 \\
\hline 388 & 316 \\
\hline 1178 & 1172 \\
\hline 1599 & 1643 \\
\hline 1627 & 1643 \\
\hline 3399 & 3430 \\
\hline 3516 & 3529 \\
\hline 3524 & 3529 \\
\hline \multicolumn{2}{|c|}{$\mathrm{Sc}\left(\mathrm{NH}_{3}\right)_{2}$} \\
\hline 31 & 28 \\
\hline 72 & 36 \\
\hline 75 & 59 \\
\hline 302 & 257 \\
\hline 361 & 302 \\
\hline 376 & 352 \\
\hline 380 & 405 \\
\hline 380 & 410 \\
\hline 411 & 439 \\
\hline 1144 & 1182 \\
\hline 1147 & 1187 \\
\hline 1617 & 1373 \\
\hline 1619 & 1632 \\
\hline 1623 & 1642 \\
\hline 1677 & 1642 \\
\hline 3394 & 3458 \\
\hline 3397 & 3462 \\
\hline 3520 & 3551 \\
\hline 3521 & 3558 \\
\hline 3524 & 3587 \\
\hline 3541 & 3591 \\
\hline \multicolumn{2}{|c|}{$\mathrm{Sc}\left(\mathrm{NH}_{3}\right)_{3}$} \\
\hline 10 & 33 \\
\hline 65 & 53 \\
\hline 86 & 70 \\
\hline 93 & 78 \\
\hline 138 & 109 \\
\hline 138 & 117 \\
\hline 263 & 273 \\
\hline 285 & 283 \\
\hline 352 & 328 \\
\hline 366 & 343 \\
\hline 383 & 378 \\
\hline 386 & 396 \\
\hline 399 & 422 \\
\hline 406 & 472 \\
\hline 476 & 490 \\
\hline 1141 & 1168 \\
\hline
\end{tabular}




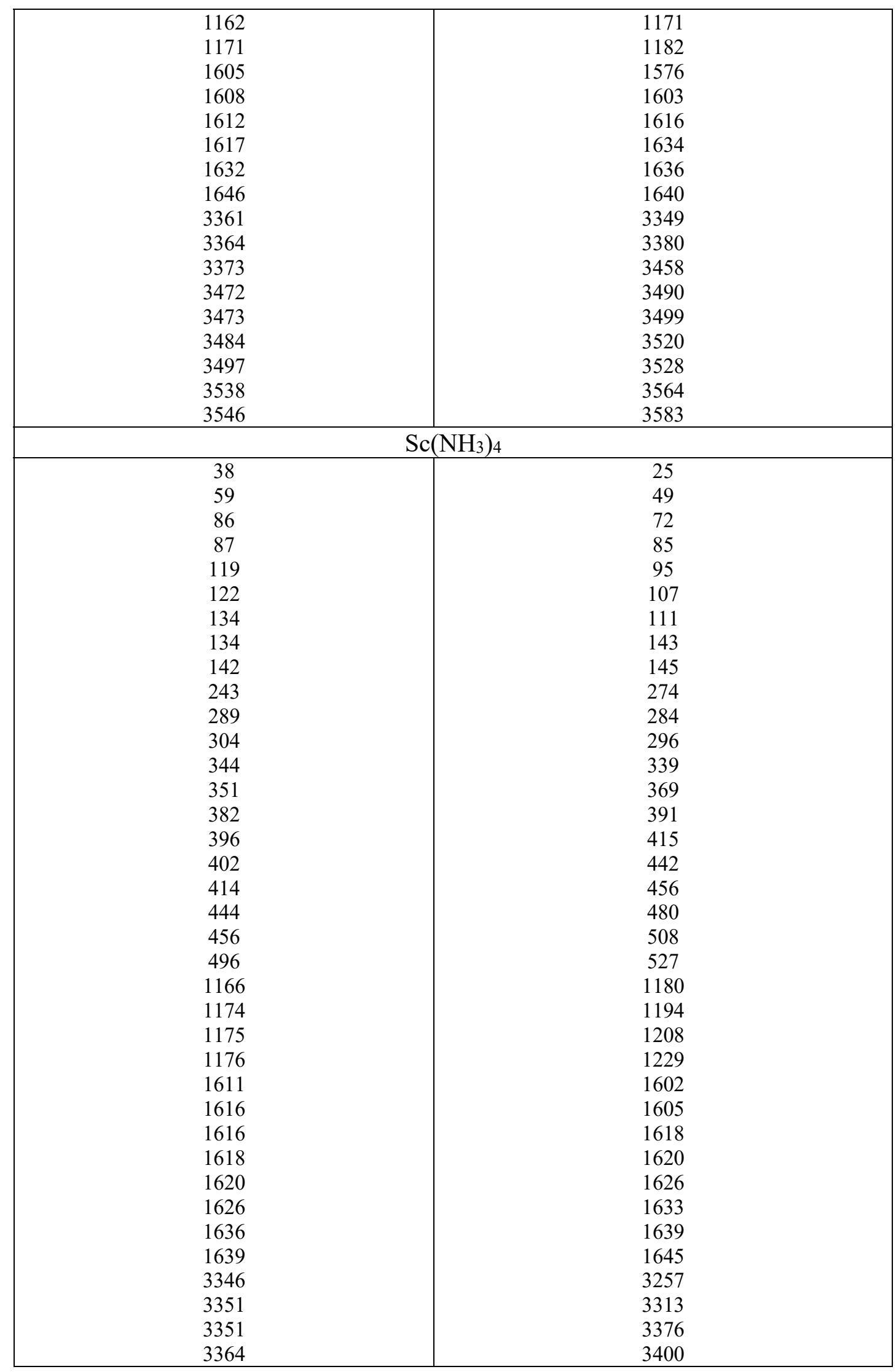




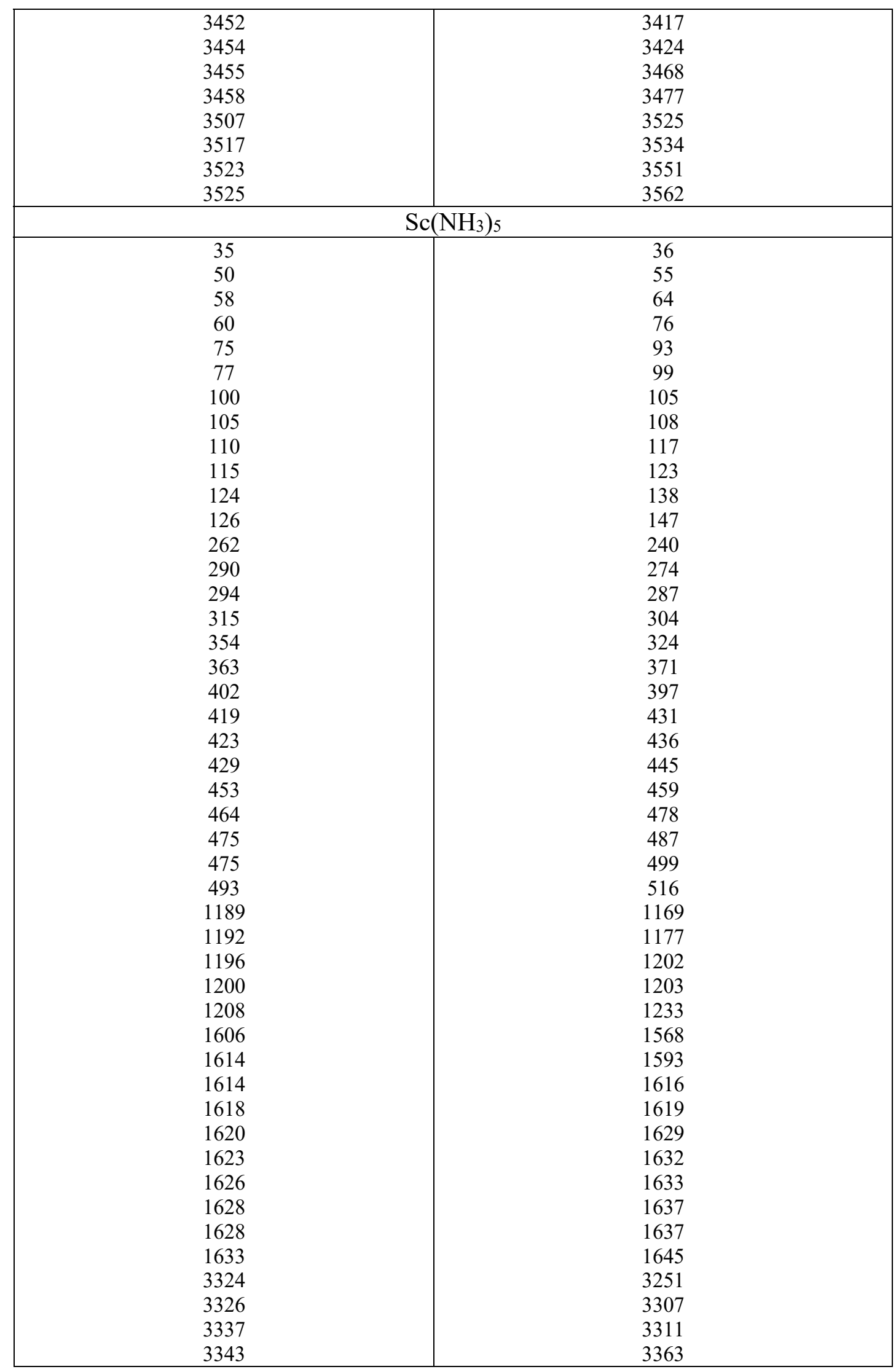




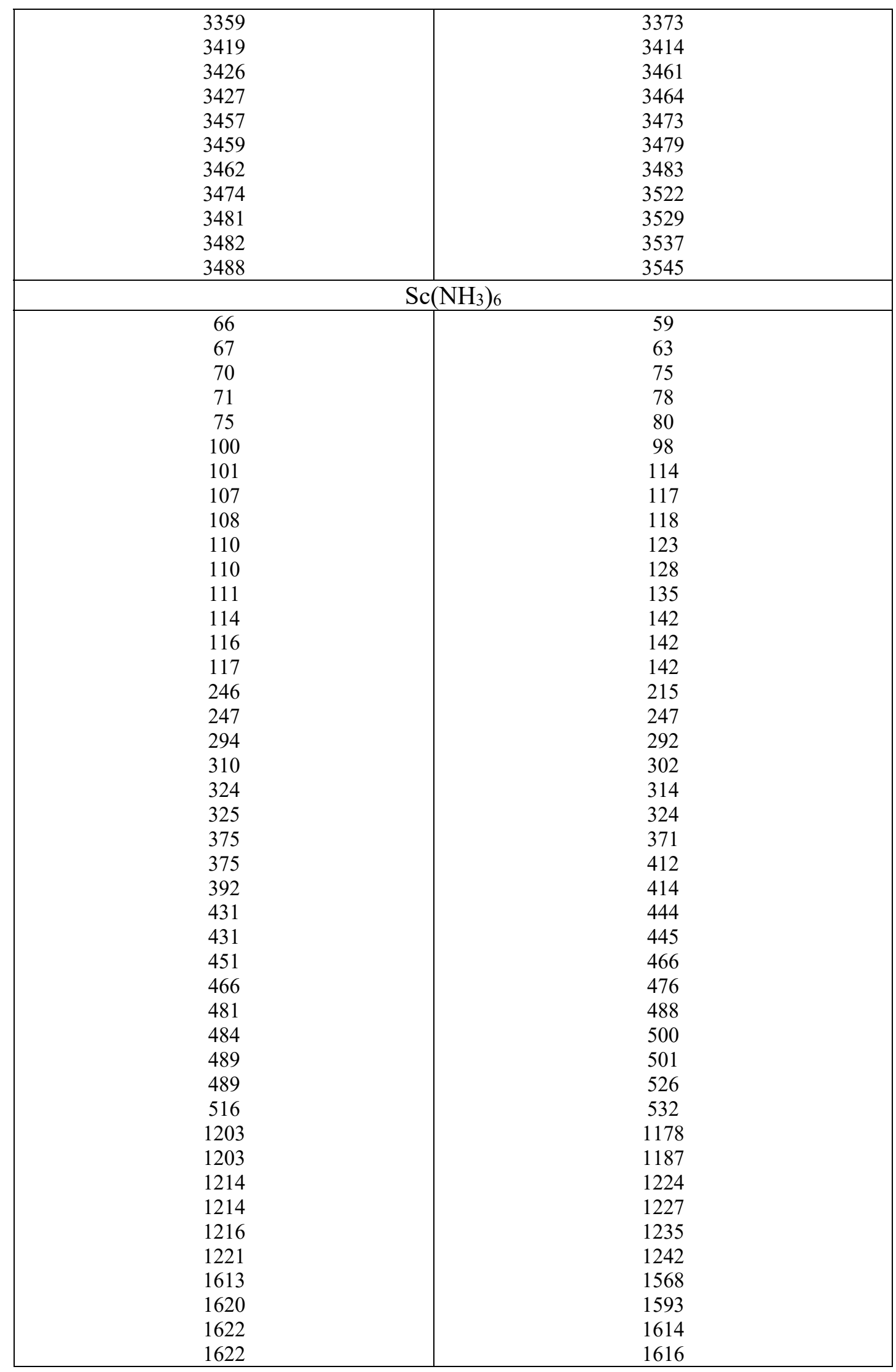




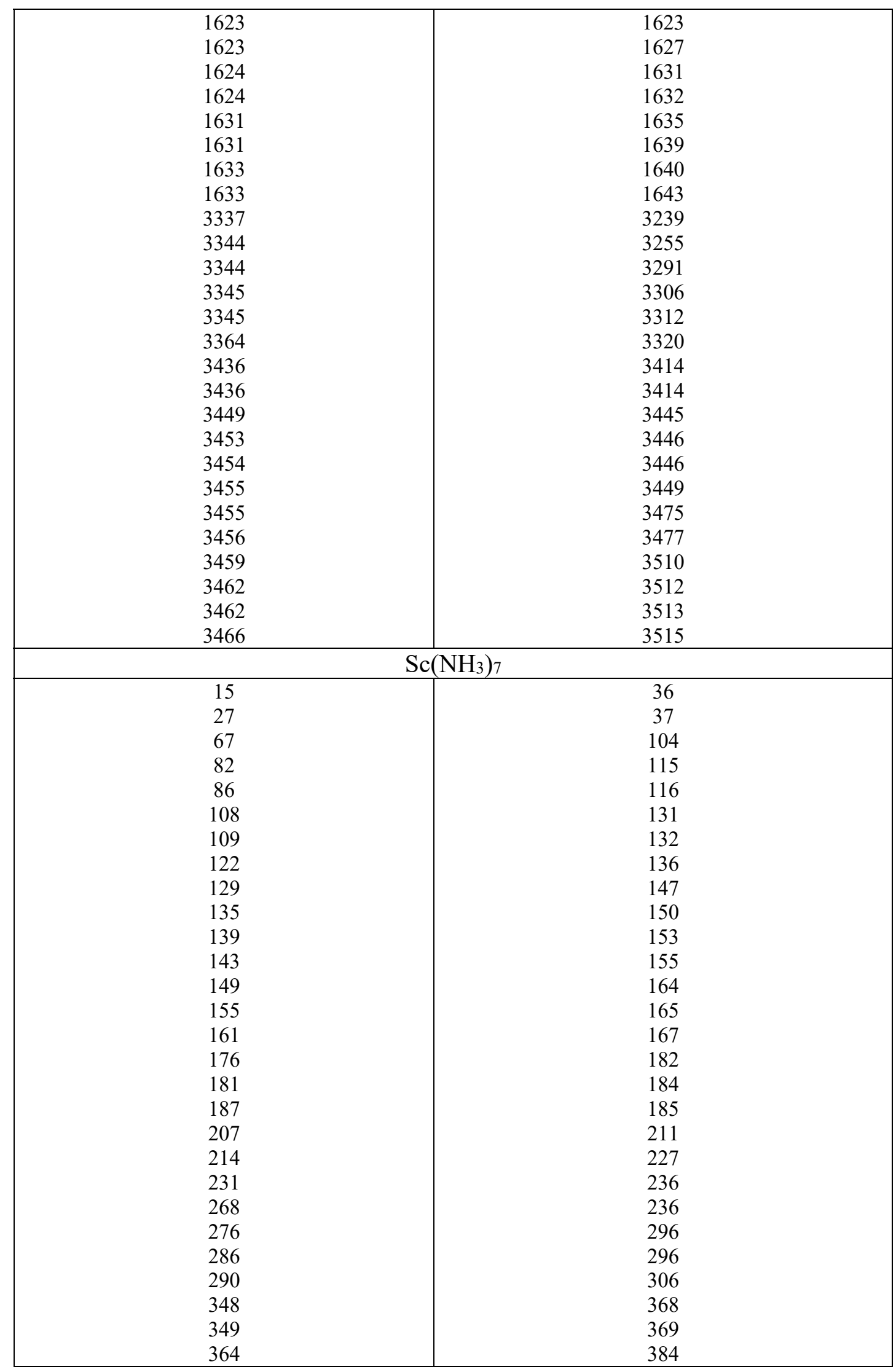




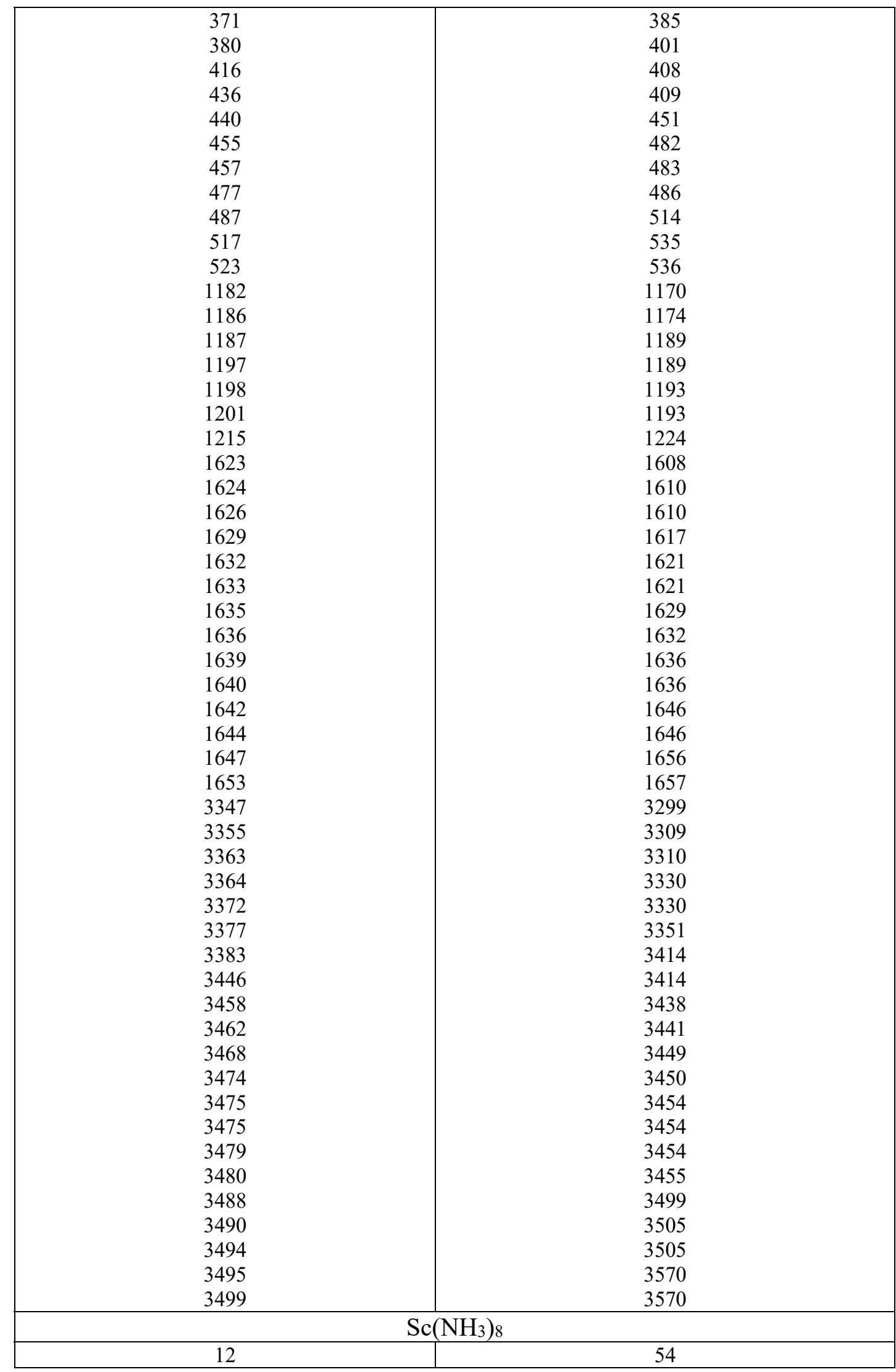




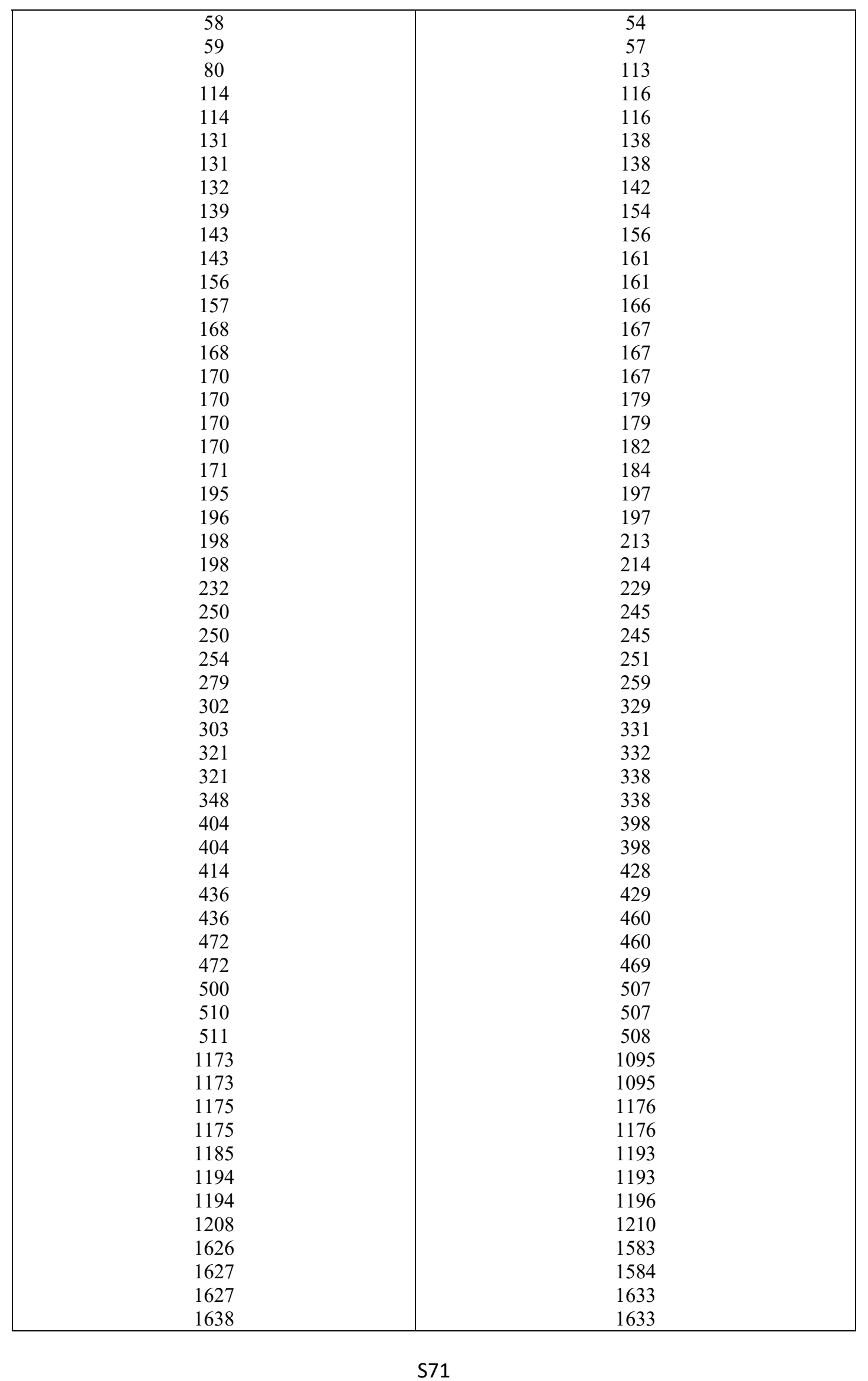




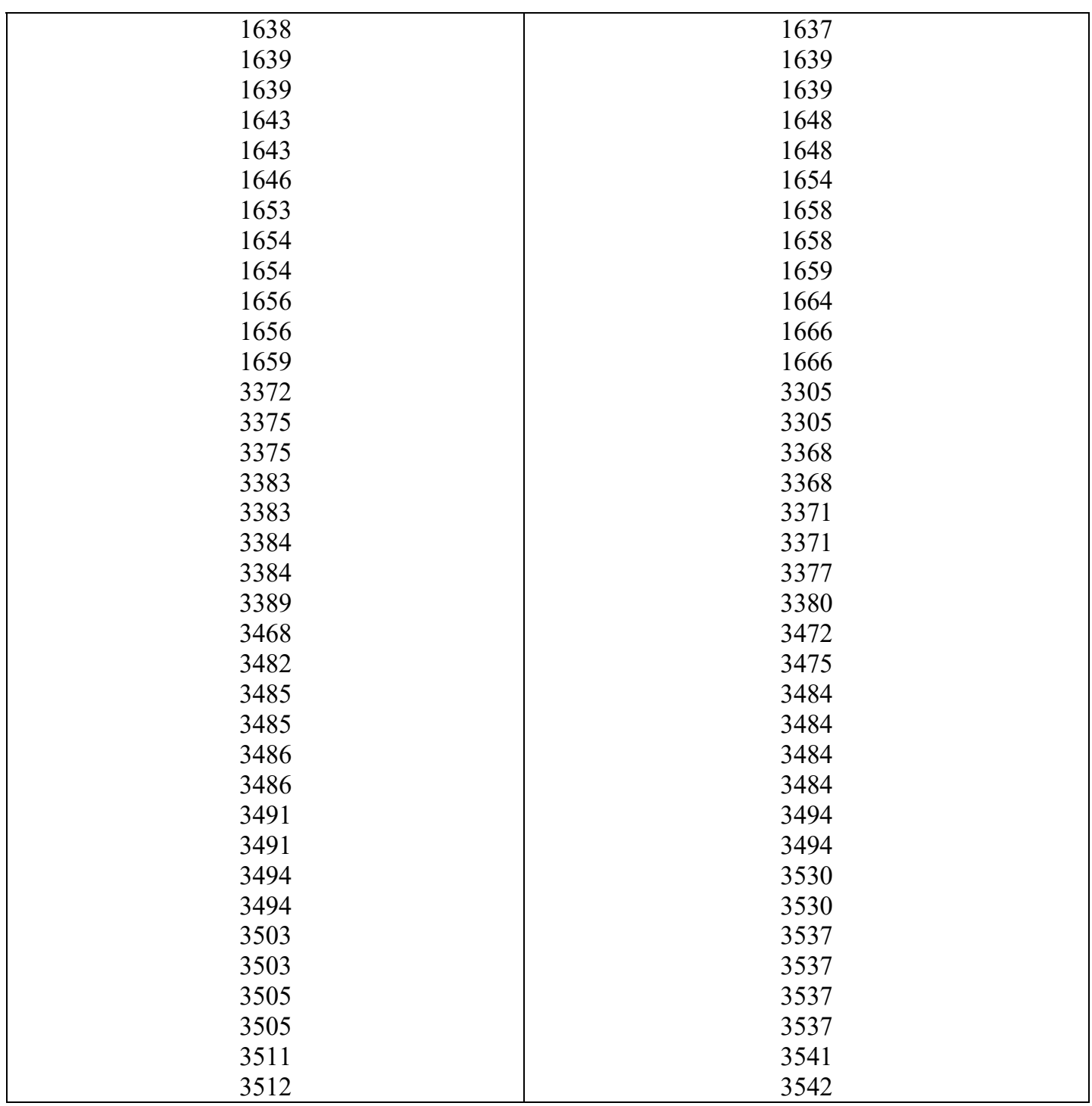


Table S24: Cartesian coordinates $(\AA)$ for the $\mathrm{Sc}\left(\mathrm{NH}_{3}\right)_{n=6,8}$ doublet spin species optimized at CAM$\mathrm{B} 3 \mathrm{LYP} / \mathrm{cc}-\mathrm{pVTZ}(\mathrm{Sc}, \mathrm{N})$ aug-cc-pVTZ $(\mathrm{H})$ constrained in $\mathrm{C}_{2 h}, \mathrm{C}_{2 v}$ symmetry used for the excitation energy calculations.

\begin{tabular}{|c|c|c|c|c|}
\hline \multicolumn{4}{|c|}{$\mathrm{Sc}\left(\mathrm{NH}_{3}\right)_{6}$} & $\mathrm{Sc}\left(\mathrm{NH}_{3}\right)_{8}$ \\
\hline $\mathrm{Sc}$ & 0.0000000 & 0.0000000 & 0.0000000 & Sc 0.0000000 .0000000 .000000 \\
\hline $\mathrm{N}$ & 0.0000000 & 1.6245610 & 1.7525050 & N 0.0000002 .1054981 .380877 \\
\hline $\mathrm{H}$ & -0.1904420 & 2.5611920 & 1.3922710 & N -2.105498 0.0000001 .380877 \\
\hline $\mathrm{H}$ & 0.9099630 & 1.6775400 & 2.2134620 & N $0.000000-2.1054981 .380877$ \\
\hline $\mathrm{N}$ & 2.3751270 & -0.2657600 & 0.0000000 & N -1.488812 $1.488812-1.380877$ \\
\hline $\mathrm{H}$ & 2.7997390 & 0.1774380 & 0.8164150 & N $1.488812-1.488812-1.380877$ \\
\hline $\mathrm{H}$ & 2.7997390 & 0.1774380 & -0.8164150 & N $1.4888121 .488812-1.380877$ \\
\hline $\mathrm{N}$ & 0.0000000 & -1.6245610 & -1.7525050 & N 2.1054980 .0000001 .380877 \\
\hline $\mathrm{H}$ & 0.6857880 & -1.4557670 & -2.4898530 & $\mathrm{~N}-1.488812-1.488812-1.380877$ \\
\hline $\mathrm{H}$ & -0.9099630 & -1.6775400 & -2.2134620 & H -0.812933 2.7017751 .235958 \\
\hline $\mathrm{N}$ & -2.3751270 & 0.2657600 & 0.0000000 & Н 0.0000001 .8636052 .371587 \\
\hline $\mathrm{H}$ & -2.7997390 & -0.1774380 & 0.8164150 & Н 0.8129332 .7017751 .235958 \\
\hline $\mathrm{H}$ & -2.6959890 & 1.2350040 & 0.0000000 & H - $2.701775-0.8129331 .235958$ \\
\hline $\mathrm{H}$ & 2.6959890 & -1.2350040 & 0.0000000 & H - 1.8636050 .0000002 .371587 \\
\hline $\mathrm{H}$ & -0.6857880 & 1.4557670 & 2.4898530 & H -2.701775 0.8129331 .235958 \\
\hline $\mathrm{H}$ & -2.7997390 & -0.1774380 & -0.8164150 & Н $0.812933-2.7017751 .235958$ \\
\hline $\mathrm{H}$ & 0.1904420 & -2.5611920 & -1.3922710 & Н $0.000000-1.8636052 .371587$ \\
\hline $\mathrm{N}$ & 0.0000000 & -1.6245610 & 1.7525050 & H - $0.812933-2.7017751 .235958$ \\
\hline $\mathrm{H}$ & 0.1904420 & -2.5611920 & 1.3922710 & Н 2.7017750 .8129331 .235958 \\
\hline $\mathrm{H}$ & -0.9099630 & -1.6775400 & 2.2134620 & H 1.8636050 .0000002 .371587 \\
\hline $\mathrm{H}$ & 0.6857880 & -1.4557670 & 2.4898530 & Н $2.701775-0.8129331 .235958$ \\
\hline $\mathrm{N}$ & 0.0000000 & 1.6245610 & -1.7525050 & H - $1.3356132 .485274-1.235958$ \\
\hline $\mathrm{H}$ & -0.6857880 & 1.4557670 & -2.4898530 & H - $1.3177671 .317767-2.371587$ \\
\hline $\mathrm{H}$ & 0.9099630 & 1.6775400 & -2.2134620 & H - $2.4852741 .335613-1.235958$ \\
\hline $\mathrm{H}$ & -0.1904420 & 2.5611920 & -1.3922710 & $\begin{array}{rrr}\text { H } 1.335613 & -2.485274 & -1.235958 \\
\text { H } 1.317767 & -1.317767 & -2.371587 \\
\text { H } 2.485274 & -1.335613 & -1.235958 \\
\text { H } 2.485274 & 1.335613 & -1.235958 \\
\text { H } 1.317767 & 1.317767 & -2.371587 \\
\text { H } 1.335613 & 2.485274 & -1.235958 \\
\text { H }-2.485274 & -1.335613 & -1.235958 \\
\text { H }-1.317767 & -1.317767 & -2.371587 \\
\text { H }-1.335613 & -2.485274 & -1.235958 \\
\end{array}$ \\
\hline
\end{tabular}


Table S25: Electronic configurations and vertical excitation energies $\triangle \mathrm{E}(\mathrm{eV})$ for the low-lying electronic states of $\mathrm{Sc}\left(\mathrm{NH}_{3}\right)_{6}$ at CASSCF and CASPT2 using the CAM-B3LYP optimized structure of the lowest doublet state and the cc-pVTZ(Sc,N) d-aug-ccpVTZ(H) basis set. The different colors signify states with the same electronic configurations.

\begin{tabular}{|c|c|c|c|c|c|}
\hline State $^{a}$ & $\begin{array}{c}\text { Electronic } \\
\text { Configuration } \\
\end{array}$ & $\begin{array}{l}\text { Irrep } \\
\left(\mathrm{C}_{2 \mathrm{~h}}\right)^{b}\end{array}$ & $\begin{array}{c}\Delta \mathrm{E} \\
(\mathrm{CASSCF})\end{array}$ & $\begin{array}{c}\Delta \mathrm{E} \\
(\mathrm{CASPT} 2)\end{array}$ & $\Delta \Delta \mathrm{E}^{c}$ \\
\hline \multirow[t]{3}{*}{${ }^{2} \mathrm{~T}_{2 \mathrm{~g}}$} & \multirow[t]{3}{*}{$\mathrm{t}_{2 \mathrm{~g}}{ }^{1}\left({ }^{2} \mathrm{~T}_{2 \mathrm{~g}}\right) 1 \mathrm{~s}^{2}\left({ }^{1} \mathrm{~A}_{1 \mathrm{~g}}\right)$} & ${ }^{2} \mathrm{Ag}$ & 0.000 & 0.000 & 0.000 \\
\hline & & ${ }^{2} \mathrm{Ag}$ & 0.150 & 0.148 & -0.002 \\
\hline & & ${ }^{2} \mathrm{~B}_{\mathrm{g}}$ & 0.150 & 0.160 & 0.010 \\
\hline \multirow[t]{9}{*}{${ }^{4} \mathrm{~A}_{2 \mathrm{u}}+{ }^{4} \mathrm{E}_{\mathrm{u}}+{ }^{4} \mathrm{~T}_{1 \mathrm{u}}+{ }^{4} \mathrm{~T}_{2 \mathrm{u}}$} & \multirow[t]{9}{*}{$\mathrm{t}_{2 \mathrm{~g}}{ }^{1}\left({ }^{2} \mathrm{~T}_{2 \mathrm{~g}}\right) 1 \mathrm{~s}^{1}\left({ }^{2} \mathrm{~A}_{1 \mathrm{~g}}\right) 1 \mathrm{p}^{1}\left({ }^{2} \mathrm{~T}_{1 \mathrm{u}}\right)$} & ${ }^{4} \mathrm{~A}_{u}$ & 0.340 & 0.457 & 0.117 \\
\hline & & ${ }^{4} \mathrm{~B}_{\mathrm{u}}$ & 0.341 & 0.464 & 0.123 \\
\hline & & ${ }^{4} \mathrm{~B}_{\mathrm{u}}$ & 0.387 & 0.497 & 0.110 \\
\hline & & ${ }^{4} \mathrm{~A}_{u}$ & 0.476 & 0.615 & 0.139 \\
\hline & & ${ }^{4} \mathrm{~B}_{\mathrm{u}}$ & 0.476 & 0.613 & 0.136 \\
\hline & & ${ }^{4} \mathrm{~A}_{u}$ & 0.498 & 0.629 & 0.132 \\
\hline & & ${ }^{4} \mathrm{~B}_{\mathrm{u}}$ & 0.542 & 0.636 & 0.094 \\
\hline & & ${ }^{4} \mathrm{Au}_{\mathrm{u}}$ & 0.543 & 0.654 & 0.111 \\
\hline & & ${ }^{4} \mathrm{~B}_{\mathrm{u}}$ & 0.543 & 0.653 & 0.109 \\
\hline \multirow{18}{*}{$\begin{array}{l}{ }^{2} \mathrm{~A}_{2 u}+{ }^{2} \mathrm{E}_{\mathrm{u}}+{ }^{2} \mathrm{~T}_{1 u}+{ }^{2} \mathrm{~T}_{2 u} \mathrm{u} \\
+{ }^{2} \mathrm{~A}_{2 u}+{ }^{2} \mathrm{E}_{\mathrm{u}}+{ }^{2} \mathrm{~T}_{1 \mathrm{u}}+{ }^{2} \mathrm{~T}_{2 \mathrm{u}}\end{array}$} & \multirow{18}{*}{$\mathrm{t}_{2 \mathrm{~g}}{ }^{1}\left({ }^{2} \mathrm{~T}_{2 \mathrm{~g}}\right) 1 \mathrm{~s}^{1}\left({ }^{2} \mathrm{~A}_{1 \mathrm{~g}}\right) 1 \mathrm{p}^{1}\left({ }^{2} \mathrm{~T}_{1 \mathrm{u}}\right)$} & ${ }^{2} \mathrm{Au}$ & 0.349 & 0.485 & 0.137 \\
\hline & & ${ }^{2} \mathrm{~B}_{\mathrm{u}}$ & 0.349 & 0.493 & 0.144 \\
\hline & & ${ }^{2} \mathrm{~B}_{\mathrm{u}}$ & 0.410 & 0.539 & 0.129 \\
\hline & & ${ }^{2} \mathrm{~A}_{u}$ & 0.489 & 0.655 & 0.165 \\
\hline & & ${ }^{2} \mathrm{~B}_{\mathrm{u}}$ & 0.489 & 0.650 & 0.160 \\
\hline & & ${ }^{2} \mathrm{~A}_{u}$ & 0.513 & 0.670 & 0.157 \\
\hline & & ${ }^{2} \mathrm{~B}_{\mathrm{u}}$ & 0.561 & 0.677 & 0.116 \\
\hline & & ${ }^{2} \mathrm{~B}_{\mathrm{u}}$ & 0.561 & 0.693 & 0.132 \\
\hline & & ${ }^{2} \mathrm{~A}_{u}$ & 0.562 & 0.697 & 0.136 \\
\hline & & ${ }^{2} \mathrm{~A}_{u}$ & 1.019 & 1.146 & 0.127 \\
\hline & & ${ }^{2} \mathrm{~B}_{\mathrm{u}}$ & 1.019 & 1.154 & 0.135 \\
\hline & & ${ }^{2} \mathrm{Bu}_{\mathrm{u}}$ & 1.034 & 1.158 & 0.124 \\
\hline & & ${ }^{2} \mathrm{Au}_{u}$ & 1.172 & 1.308 & 0.136 \\
\hline & & ${ }^{2} \mathrm{Bu}_{\mathrm{u}}$ & 1.173 & 1.307 & 0.134 \\
\hline & & ${ }^{2} \mathrm{Au}_{u}$ & 1.174 & 1.310 & 0.137 \\
\hline & & ${ }^{2} \mathrm{~B}_{\mathrm{u}}$ & 1.184 & $1.322^{d}$ & $0.138^{e}$ \\
\hline & & ${ }^{2} \mathrm{~A}_{u}$ & 1.187 & 1.323 & 0.136 \\
\hline & & ${ }^{2} \mathrm{~B}_{\mathrm{u}}$ & 1.187 & $1.325^{d}$ & $0.138^{e}$ \\
\hline \multirow{8}{*}{$\begin{array}{c}{ }^{2} \mathrm{~A}_{1 \mathrm{~g}}+{ }^{2} \mathrm{E}_{\mathrm{g}}+{ }^{2} \mathrm{~T}_{1 \mathrm{~g}}+{ }^{2} \mathrm{~T}_{2 \mathrm{~g}} \\
{ }^{2} \mathrm{~A}_{1 \mathrm{~g}}+{ }^{2} \mathrm{E}_{\mathrm{g}}+{ }^{2} \mathrm{~T}_{1 \mathrm{~g}}+{ }^{2} \mathrm{~T}_{2 \mathrm{~g}}+{ }^{2} \mathrm{~T}_{1 \mathrm{~g}}+{ }^{2} \mathrm{~T}_{2 \mathrm{~g}} \\
{ }^{2} \mathrm{~T}_{2 \mathrm{~g}}\end{array}$} & \multirow{8}{*}{$\begin{array}{c}\left.\mathrm{t}_{2 \mathrm{~g}}{ }^{1}{ }^{(} \mathrm{T}_{2 \mathrm{~g}}\right) 1 \mathrm{p}^{2}\left({ }^{3} \mathrm{~T}_{2 \mathrm{~g}} \sim{ }^{3} \mathrm{P}\right) \\
\left.\mathrm{t}_{2 \mathrm{~g}}{ }^{2}{ }^{2} \mathrm{~T}_{2 \mathrm{~g}}\right) 1 \mathrm{p}^{2}\left({ }^{1} \mathrm{~T}_{2 \mathrm{~g}}+{ }^{1} \mathrm{E}_{\mathrm{g}} \sim{ }^{1} \mathrm{D}\right) \\
\mathrm{t}_{2 \mathrm{~g}}{ }^{1}\left({ }^{2} \mathrm{~T}_{2 \mathrm{~g}}\right) 1 \mathrm{p}^{2}\left({ }^{1} \mathrm{~A}_{1 \mathrm{~g}} \sim \sim^{1} \mathrm{~S}\right)\end{array}$} & ${ }^{2} \mathrm{~B}_{\mathrm{g}}$ & 0.915 & 1.198 & 0.283 \\
\hline & & ${ }^{2} \mathrm{Ag}$ & 0.916 & 1.204 & 0.288 \\
\hline & & ${ }^{2} \mathrm{Ag}$ & 0.995 & 1.286 & 0.291 \\
\hline & & ${ }^{2} \mathrm{~B}_{\mathrm{g}}$ & 0.996 & 1.267 & 0.271 \\
\hline & & ${ }^{2} \mathrm{Ag}$ & 0.996 & 1.260 & 0.263 \\
\hline & & ${ }^{2} \mathrm{Bg}_{\mathrm{g}}$ & 1.042 & 1.342 & 0.301 \\
\hline & & ${ }^{2} \mathrm{~B}_{\mathrm{g}}$ & 1.085 & 1.365 & 0.281 \\
\hline & & ${ }^{2} \mathrm{Ag}$ & 1.085 & 1.361 & 0.276 \\
\hline
\end{tabular}




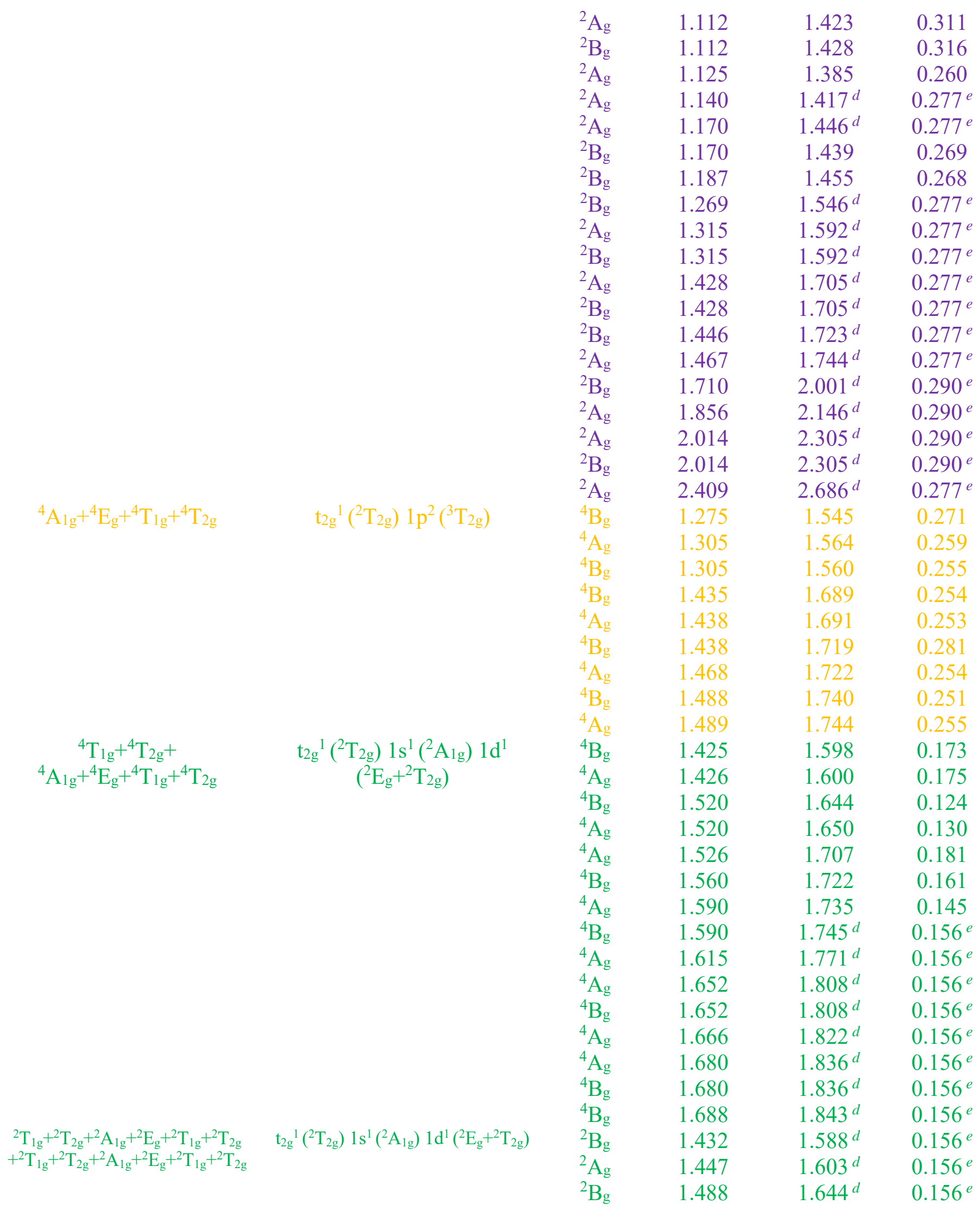




\begin{tabular}{llll}
${ }^{2} \mathrm{Ag}$ & 1.489 & $1.645^{d}$ & $0.156^{e}$ \\
${ }^{2} \mathrm{Ag}$ & 1.538 & $1.694^{d}$ & $0.156^{e}$ \\
${ }^{2} \mathrm{Bg}$ & 1.538 & $1.694^{d}$ & $0.156^{e}$ \\
${ }^{2} \mathrm{Ag}$ & 1.547 & $1.703^{d}$ & $0.156^{e}$ \\
${ }^{2} \mathrm{Bg}$ & 1.586 & $1.742^{d}$ & $0.156^{e}$ \\
${ }^{2} \mathrm{Ag}$ & 1.609 & $1.765^{d}$ & $0.156^{e}$ \\
${ }^{2} \mathrm{Bg}$ & 1.609 & $1.765^{d}$ & $0.156^{e}$ \\
${ }^{2} \mathrm{Ag}$ & 1.638 & $1.794^{d}$ & $0.156^{e}$ \\
${ }^{2} \mathrm{Ag}$ & 1.678 & $1.834^{d}$ & $0.156^{e}$ \\
${ }^{2} \mathrm{Bg}$ & 1.678 & $1.834^{d}$ & $0.156^{e}$ \\
${ }^{2} \mathrm{Ag}$ & 1.691 & $1.847^{d}$ & $0.156^{e}$ \\
${ }^{2} \mathrm{Ag}$ & 1.704 & $1.860^{d}$ & $0.156^{e}$ \\
${ }^{2} \mathrm{Bg}$ & 1.704 & $1.860^{d}$ & $0.156^{e}$ \\
${ }^{2} \mathrm{Bg}$ & 2.237 & $2.393^{d}$ & $0.156^{e}$ \\
${ }^{2} \mathrm{Ag}$ & 2.237 & $2.393^{d}$ & $0.156^{e}$ \\
${ }^{2} \mathrm{Bg}$ & 2.267 & $2.423^{d}$ & $0.156^{e}$ \\
${ }^{2} \mathrm{Ag}$ & 2.267 & $2.423^{d}$ & $0.156^{e}$ \\
${ }^{2} \mathrm{Bg}$ & 2.282 & $2.438^{d}$ & $0.156^{e}$ \\
${ }^{2} \mathrm{Ag}$ & 2.382 & $2.538^{d}$ & $0.156^{e}$ \\
${ }^{2} \mathrm{Bg}$ & 2.391 & $2.547^{d}$ & $0.156^{e}$ \\
${ }^{2} \mathrm{Ag}$ & 2.391 & $2.547^{d}$ & $0.156^{e}$ \\
${ }^{2} \mathrm{Ag}$ & 2.402 & $2.558^{d}$ & $0.156^{e}$ \\
${ }^{2} \mathrm{Ag}$ & 2.426 & $2.582^{d}$ & $0.156^{e}$ \\
${ }^{2} \mathrm{Bg}$ & 2.426 & $2.582^{d}$ & $0.156^{e}$ \\
${ }^{2} \mathrm{Bg}$ & 2.437 & $2.593^{d}$ & $0.156^{e}$ \\
${ }^{2} \mathrm{Ag}$ & 2.448 & $2.604^{d}$ & $0.156^{e}$ \\
${ }^{2} \mathrm{Bg}$ & 2.448 & $2.604^{d}$ & $0.156^{e}$ \\
\hline
\end{tabular}

${ }^{a}$ Electronic terms under $\mathrm{O}_{\mathrm{h}}$ obtained based on the electronic configuration.

${ }^{b}$ Irreducible representation under $\mathrm{C}_{2} \mathrm{~h}$.

${ }^{c} \Delta \Delta \mathrm{E}=\Delta \mathrm{E}(\mathrm{CASPT} 2)-\Delta \mathrm{E}(\mathrm{CASSCF})$.

${ }^{d}$ Estimated values obtained as $\Delta \mathrm{E}(\mathrm{CASPT} 2)=\Delta \mathrm{E}(\mathrm{CASSCF})+\delta \mathrm{E}$ (see footnote e).

${ }^{e} \delta \mathrm{E}$ value obtained as average $\Delta \Delta \mathrm{E}$ for states with identical electronic configuration. 
Table S26: Electronic configurations and vertical excitation energies $\Delta \mathrm{E}(\mathrm{eV})$ for the low-lying electronic states of $\mathrm{Sc}\left(\mathrm{NH}_{3}\right)_{8}$ at CASSCF and CASPT2 using the CAM-B3LYP optimized structure of the lowest doublet state and the cc-pVDZ(Sc,N) d-aug-ccpVDZ(H) basis set. The different colors signify states with the same electronic configurations.

\begin{tabular}{|c|c|c|c|c|c|}
\hline State $^{a}$ & $\begin{array}{c}\text { Electronic } \\
\text { Configuration }^{b}\end{array}$ & $\begin{array}{l}\text { Irrep } \\
\left(\mathrm{C}_{2 \mathrm{v}}\right)^{c}\end{array}$ & $\begin{array}{c}\Delta \mathrm{E} \\
(\mathrm{CASSCF})\end{array}$ & $\begin{array}{c}\Delta \mathrm{E} \\
(\mathrm{CASPT} 2)\end{array}$ & $\Delta \Delta \mathrm{E}^{d}$ \\
\hline${ }^{2} \mathrm{~A}_{1}$ & $3 d_{a_{1}}^{1} 1 s_{a_{1}}^{2}$ & ${ }^{2} \mathrm{~A}_{1}$ & 0.000 & 0.000 & 0.000 \\
\hline${ }^{4} \mathrm{~B}_{2}$ & $3 d_{a_{1}}^{1} 1 s_{a_{1}}^{1} 1 p_{b_{2}}^{1}$ & ${ }^{4} \mathrm{~A}_{1}$ & 0.361 & 0.427 & 0.066 \\
\hline${ }^{2} \mathrm{~B}_{2}$ & $3 d_{a_{1}}^{1} 1 s_{a_{1}}^{1} 1 p_{b_{2}}^{1}$ & ${ }^{2} \mathrm{~A}_{1}$ & 0.384 & 0.433 & 0.049 \\
\hline \multirow[t]{2}{*}{${ }^{2} E_{1}$} & $3 d_{a_{1}}^{1} 1 s_{a_{1}}^{1} 1 p_{e_{1}}^{1}$ & ${ }^{2} \mathrm{~B}_{1}$ & 0.479 & 0.525 & 0.045 \\
\hline & $3 d_{a_{1}}^{1} 1 s_{a_{1}}^{1} 1 p_{e_{1}}^{1}$ & ${ }^{2} \mathrm{~B}_{2}$ & 0.479 & 0.525 & 0.045 \\
\hline \multirow[t]{2}{*}{${ }^{4} \mathrm{E}_{1}$} & $3 d_{a_{1}}^{1} 1 s_{a_{1}}^{1} 1 p_{e_{1}}^{1}$ & ${ }^{4} \mathrm{~B}_{1}$ & 0.476 & 0.539 & 0.064 \\
\hline & $3 d_{a_{1}}^{1} 1 s_{a_{1}}^{1} 1 p_{e_{1}}^{1}$ & ${ }^{4} \mathrm{~B}_{2}$ & 0.476 & 0.539 & 0.064 \\
\hline \multirow[t]{2}{*}{${ }^{2} \mathrm{E}_{2}$} & $3 d_{e_{2}}^{1} 1 s_{a_{1}}^{2}$ & ${ }^{2} \mathrm{~A}_{1}$ & 0.840 & 0.873 & 0.033 \\
\hline & $3 d_{e_{2}}^{1} 1 s_{a_{1}}^{2}$ & ${ }^{2} \mathrm{~A}_{2}$ & 0.840 & 0.903 & 0.062 \\
\hline \multirow[t]{2}{*}{${ }^{2} \mathrm{E}_{3}$} & $3 d_{e_{3}}^{1} 1 s_{a_{1}}^{2}$ & ${ }^{2} \mathrm{~B}_{1}$ & 1.175 & 1.241 & 0.065 \\
\hline & $3 d_{e_{3}}^{1} 1 s_{a_{1}}^{2}$ & ${ }^{2} \mathrm{~B}_{2}$ & 1.175 & 1.241 & 0.065 \\
\hline \multirow[t]{2}{*}{${ }^{4} E_{2}$} & $3 d_{e_{2}}^{1} 1 s_{a_{1}}^{1} 1 p_{b_{2}}^{1}$ & ${ }^{4} \mathrm{~A}_{1}$ & 1.174 & 1.288 & 0.114 \\
\hline & $3 d_{e_{2}}^{1} 1 s_{a_{1}}^{1} 1 p_{b_{2}}^{1}$ & ${ }^{4} \mathrm{~A}_{2}$ & 1.174 & 1.323 & 0.149 \\
\hline \multirow[t]{2}{*}{${ }^{2} \mathrm{E}_{2}$} & $3 d_{e_{2}}^{1} 1 s_{a_{1}}^{1} 1 p_{b_{2}}^{1}$ & ${ }^{2} \mathrm{~A}_{1}$ & 1.185 & 1.292 & 0.107 \\
\hline & $3 d_{e_{2}}^{1} 1 s_{a_{1}}^{1} 1 p_{b_{2}}^{1}$ & ${ }^{2} \mathrm{~A}_{2}$ & 1.185 & 1.319 & 0.134 \\
\hline \multirow[t]{4}{*}{${ }^{2}\left(\mathrm{E}_{1}+\mathrm{E}_{3}\right)$} & $3 d_{e_{2}}^{1} 1 s_{a_{1}}^{1} 1 p_{e_{1}}^{1}$ & ${ }^{2} \mathrm{~B}_{1}$ & 1.357 & 1.425 & 0.068 \\
\hline & $3 d_{e_{2}}^{1} 1 s_{a_{1}}^{1} 1 p_{e_{1}}^{1}$ & ${ }^{2} \mathrm{~B}_{2}$ & 1.357 & 1.425 & 0.068 \\
\hline & $3 d_{e_{2}}^{1} 1 s_{a_{1}}^{1} 1 p_{e_{1}}^{1}$ & ${ }^{2} \mathrm{~B}_{1}$ & 1.359 & 1.427 & 0.068 \\
\hline & $3 d_{e_{2}}^{1} 1 s_{a_{1}}^{1} 1 p_{e_{1}}^{1}$ & ${ }^{2} \mathrm{~B}_{2}$ & 1.359 & 1.427 & 0.068 \\
\hline \multirow[t]{4}{*}{${ }^{4}\left(\mathrm{E}_{1}+\mathrm{E}_{3}\right)$} & $3 d_{e_{2}}^{1} 1 s_{a_{1}}^{1} 1 p_{e_{1}}^{1}$ & ${ }^{4} \mathrm{~B}_{1}$ & 1.355 & 1.436 & 0.081 \\
\hline & $3 d_{e_{2}}^{1} 1 s_{a_{1}}^{1} 1 p_{e_{1}}^{1}$ & ${ }^{4} \mathrm{~B}_{2}$ & 1.355 & 1.436 & 0.081 \\
\hline & $3 d_{e_{2}}^{1} 1 s_{a_{1}}^{1} 1 p_{e_{1}}^{1}$ & ${ }^{4} \mathrm{~B}_{1}$ & 1.356 & 1.437 & 0.080 \\
\hline & $3 d_{e_{2}}^{1} 1 s_{a_{1}}^{1} 1 p_{e_{1}}^{1}$ & ${ }^{4} \mathrm{~B}_{2}$ & 1.356 & 1.437 & 0.080 \\
\hline \multirow[t]{2}{*}{${ }^{2} \mathrm{E}_{3}$} & $3 d_{a_{1}}^{1} 1 p_{b_{2}}^{1} 1 p_{e_{1}}^{1}$ & ${ }^{2} \mathrm{~B}_{1}$ & 1.333 & 1.453 & 0.120 \\
\hline & $3 d_{a_{1}}^{1} 1 p_{b_{2}}^{1} 1 p_{e_{1}}^{1}$ & ${ }^{2} \mathrm{~B}_{2}$ & 1.333 & 1.453 & 0.120 \\
\hline \multirow[t]{2}{*}{${ }^{4} \mathrm{E}_{3}$} & $3 d_{a_{1}}^{1} 1 p_{b_{2}}^{1} 1 p_{e_{1}}^{1}$ & ${ }^{4} \mathrm{~B}_{1}$ & 1.308 & 1.456 & 0.148 \\
\hline & $3 d_{a_{1}}^{1} 1 p_{b_{2}}^{1} 1 p_{e_{1}}^{1}$ & ${ }^{4} \mathrm{~B}_{2}$ & 1.308 & 1.456 & 0.148 \\
\hline${ }^{2} \mathrm{~A}_{2}$ & $3 d_{a_{1}}^{1} 1 p_{e_{1}}^{1} 1 p_{e_{1}}^{1}$ & ${ }^{2} \mathrm{~A}_{2}$ & 1.347 & 1.494 & 0.147 \\
\hline${ }^{4} \mathrm{~A}_{2}$ & $3 d_{a_{1}}^{1} 1 p_{e_{1}}^{1} 1 p_{e_{1}}^{1}$ & ${ }^{4} \mathrm{~A}_{2}$ & 1.333 & 1.507 & 0.174 \\
\hline \multirow[t]{2}{*}{${ }^{4} \mathrm{E}_{1}$} & $3 d_{e_{3}}^{1} 1 s_{a_{1}}^{1} 1 p_{b_{2}}^{1}$ & ${ }^{4} \mathrm{~B}_{1}$ & 1.529 & 1.659 & 0.130 \\
\hline & $3 d_{e_{3}}^{1} 1 s_{a_{1}}^{1} 1 p_{b_{2}}^{1}$ & ${ }^{4} \mathrm{~B}_{2}$ & 1.529 & 1.659 & 0.130 \\
\hline \multirow[t]{2}{*}{${ }^{2} \mathrm{E}_{1}$} & $3 d_{e_{3}}^{1} 1 s_{a_{1}}^{1} 1 p_{b_{2}}^{1}$ & ${ }^{2} \mathrm{~B}_{1}$ & 1.545 & 1.671 & 0.126 \\
\hline & $3 d_{e_{3}}^{1} 1 s_{a_{1}}^{1} 1 p_{b_{2}}^{1}$ & ${ }^{2} \mathrm{~B}_{2}$ & 1.545 & 1.671 & 0.126 \\
\hline
\end{tabular}


${ }^{a}$ Electronic terms under $\mathrm{D}_{4 d}$ obtained based on the electronic configuration.

${ }^{b} 1 \mathrm{~s}, 1 \mathrm{p}$ notation is for diffuse outer $\mathrm{s}, \mathrm{p}, \mathrm{d}$ orbital respectively.

${ }^{c}$ Irreducible representation under $\mathrm{C}_{2 v}$.

${ }^{d} \Delta \Delta \mathrm{E}=\Delta \mathrm{E}(\mathrm{CASPT} 2)-\Delta \mathrm{E}(\mathrm{CASSCF})$. 